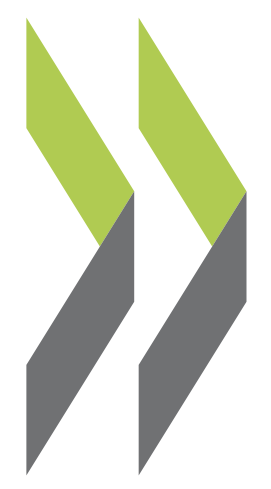

OECD Social, Employment and Migration Working Papers No. 124

\title{
Is the European Welfare State Really More
}

Expensive? Indicators on

Social Spending, 1980-2012; and a Manual to the OECD Social Expenditure Database (SOCX)

Willem Adema, Pauline Fron, Maxime Ladaique 


\title{
DIRECTORATE FOR EMPLOYMENT, LABOUR AND SOCIAL AFFAIRS
}

\section{OECD SOCIAL, EMPLOYMENT AND MIGRATION WORKING PAPERS}

\author{
www.oecd.org/els/workingpapers
}

This series is designed to make available to a wider readership selected labour market, social policy and migration studies prepared for use within the OECD. Authorship is usually collective, but principal writers are named. The papers are generally available only in their original language - English or French - with a summary in the other.

Comment on the series is welcome, and should be sent to the Directorate for Employment, Labour and Social Affairs, 2, rue André-Pascal, 75775 PARIS CEDEX 16, France.

The opinions expressed and arguments employed here are the responsibility of the author(s) and do not necessarily reflect those of the OECD.

\section{Applications for permission to reproduce or translate all or part of this material should be made to:}

\author{
Head of Publications Service \\ OECD \\ 2, rue André-Pascal \\ 75775 Paris, CEDEX 16 \\ France
}

Copyright OECD 2011 


\section{SUMMARY}

Part I of this paper first presents information on trends and composition of social expenditure as in the OECD Social Expenditure database for the years 1980 - 2007. Over this period, public social expenditure as a percentage of GDP, on average across OECD, increased from $15.6 \%$ to $19.2 \%$. Public pension spending (6.4\% of GDP) and public health expenditure (5.8\% of GDP) are the largest social spending items.

Detailed SOCX data is not available for the period after 2007. However, the public social expenditure series as in SOCX was extended to 2008, 2009 and 2010 using available information on national aggregates and public social spending aggregates were estimated for 2011 and 2012. These data suggest that on average across the OECD, public social spending as a percentage of GDP increased by 3 percentage points since 2007 to $22.5 \%$ in 2009 . Since then, this spending ratio is estimated to have fallen to just over $22 \%$ of GDP in 2012.

Part I also presents social expenditure indicators that account for the effects of the tax system as well as indicators on private social expenditure. Including both of these features alters country rankings by level of social spending and leads to a convergence of spending-to-GDP ratios across countries. Based on this broader measure net total social expenditure as a percent of GDP at factor costs in 2007 was highest in France and Belgium, at 30\% of GDP, and between 22 and 28\% of GDP in Austria, Canada, Denmark, Finland, Italy, Japan, the Netherlands, Portugal, the United Kingdom and the United States.

Part II of this paper presents the OECD SOCX Manual. It starts with a discussion of methodological, classification and data issues regarding the gross spending items as in SOCX. It also looks at the methodological aspects of measuring net social expenditure, and presents information on how relevant estimates were derived. Accounting for the effect of the tax system and private social expenditure leads to greater similarity in social expenditure-to-GDP ratios across countries and to a reassessment of the magnitude of welfare states. After accounting for the impact of taxation and private benefits, social expenditure amounts to over $30 \%$ of GDP at factor cost in Belgium and France; social expenditure also ranges within a few percentage points of each other in Austria, Canada, Denmark, Finland, Italy, Japan, the Netherlands, Portugal, the United Kingdom and the United States. 


\section{RÉSUMÉ}

La Partie I de ce document présente tout d'abord des informations sur les tendances et la composition des dépenses sociales issues de la base de données OCDE sur les dépenses sociales pour les années 1980 2007. Durant cette période, les dépenses sociales publiques en pourcentage du PIB ont augmenté en moyenne de $15,6 \%$ à $19,2 \%$ dans les pays de l'OCDE. Les dépenses de retraite publiques $(6,4 \% \mathrm{du} P \mathrm{PIB})$ et les dépenses de santé publique (5,8\% du PIB) sont les plus grandes catégories de dépenses sociales.

Les données détaillées SOCX ne sont pas disponibles pour la période après 2007. Cependant les données de dépenses sociales publiques telles que dans SOCX ont été étendues à 2008, 2009 et 2010 en utilisant les informations disponibles sur les agrégats nationaux, et les agrégats des dépenses sociales publiques ont été estimés pour 2011 et 2012. Ces données montrent que les dépenses sociales publiques ont cru de 3 points de pourcentage à partir de 2007 pour atteindre $22.5 \%$ en 2009 en moyenne dans les pays de l'OCDE. On estime ensuite que ce ratio baisse jusque 22\% du PIB en 2012.

La Partie I présente également des indicateurs de dépenses sociales tenant compte des effets du système fiscal et ainsi que des indicateurs sur les dépenses sociales privées. La prise en compte de ces deux effets modifie le classement des pays selon le niveau de dépenses sociales et conduit à une convergence des ratios entre les niveaux des dépenses sociales et le PIB entre les pays. Basées sur cette mesure plus large, les dépenses sociales totales nettes en pourcentage du PIB aux coûts des facteurs atteignent $30 \%$ du PIB en 2007 pour les plus élevées, en France et en Belgique, et varient entre 22 et $28 \%$ du PIB en Autriche, au Canada, au Danemark, en Finlande, en Italie, au Japon, aux Pays-Bas, au Portugal, au Royaume-Uni et aux États-Unis.

La Partie II de ce document présente le manuel SOCX de l'OCDE, avec tout d'abord une discussion sur des questions méthodologiques, sur la classification des dépenses brutes telles que présentées dans SOCX. Les aspects méthodologiques de la mesure de dépenses sociales nettes sont ensuite présentés, notamment avec des informations sur la façon dont les estimations ont été dérivées. La prise en compte des prestations sociales privées et de l'impact de la fiscalité sur les dépenses sociales a pour effet d'égaliser les ratios entre les niveaux des dépenses sociales et le PIB. Après la prise en compte des prestations sociales privées et de l'impact de la fiscalité, les dépenses sociales atteignent plus de $30 \%$ du PIB aux coûts des facteurs en Belgique et en France; enfin les écarts entre les dépenses sociales en Autriche, Canada, Danemark, Finlande, Italie, Japon, Pays-Bas, Portugal, Royaume-Uni et aux États-Unis ne sont que de quelques points de pourcentage. 


\section{TABLE OF CONTENTS}

SUMMARY …

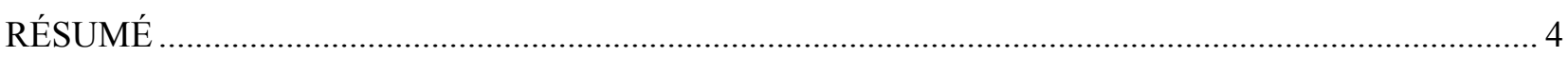

IS THE EUROPEAN WELFARE STATE REALLY MORE EXPENSIVE? …............................................. 8

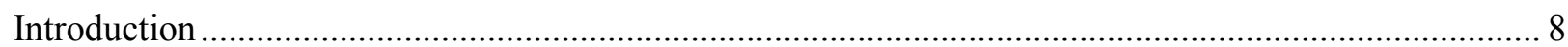

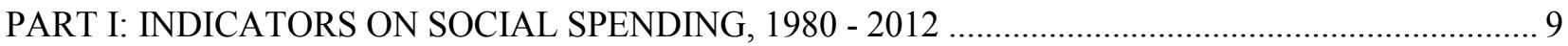

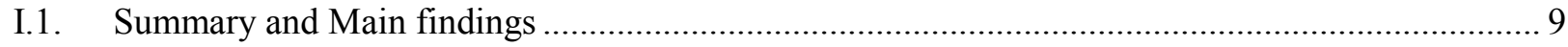

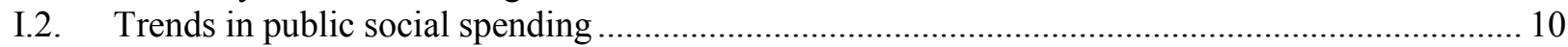

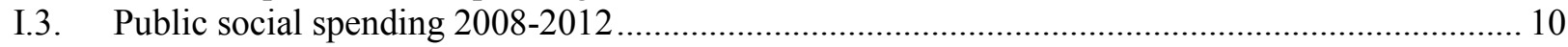

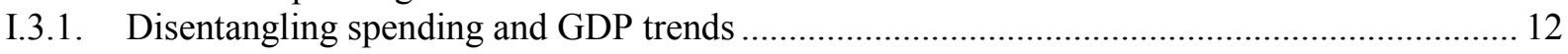

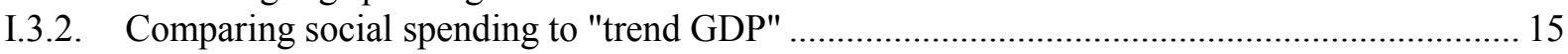

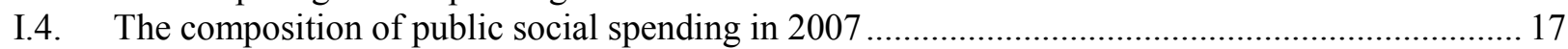

I.4.1. Trends in social expenditures 2007-2012 by broad social policy area ............................... 19

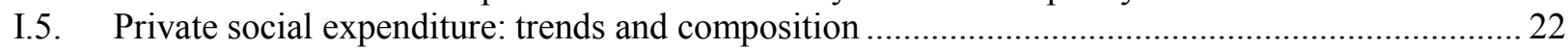

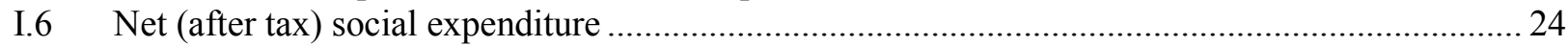

I.6.1. The value of direct taxation of transfer income in aggregate terms ........................................2 25

I.6.2. The importance of indirect taxation of consumption out of benefit income............................. 26

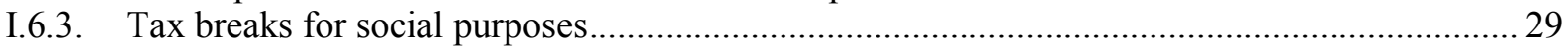

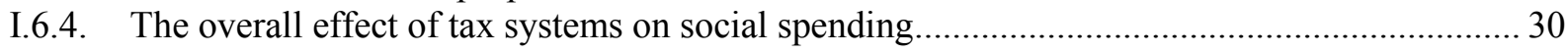

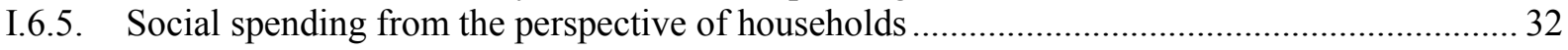

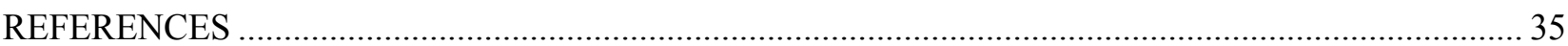

ANNEX I.1: ESTIMATING PUBLIC SOCIAL EXPENDITURE 2008-2012 - SOURCES AND

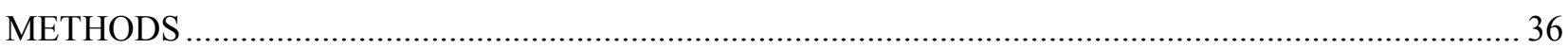

ANNEX I.2: ADDITIONAL NET SOCIAL EXPENDITURE INDICATORS: ..................................... 47

A.I.2.1: Individual country data : 2001, 2003, 2005 and 2007.......................................................... 47

A.I.2.2. Net Social Expenditure Indicators Related to GDP at Market Prices and

National Income, 2007

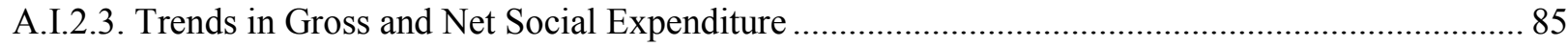




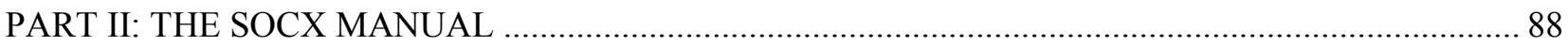

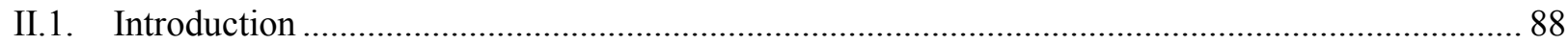

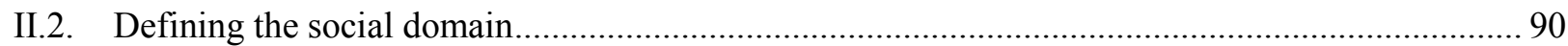

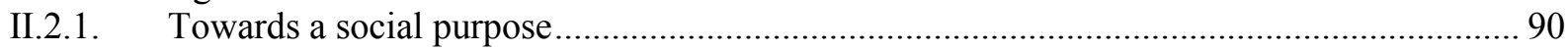

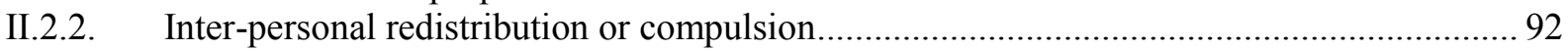

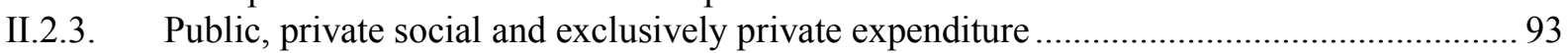

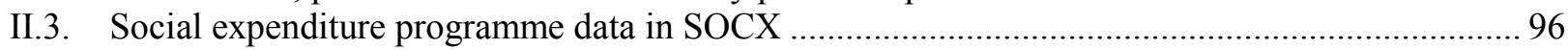

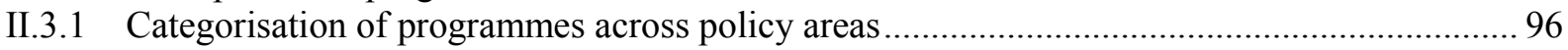

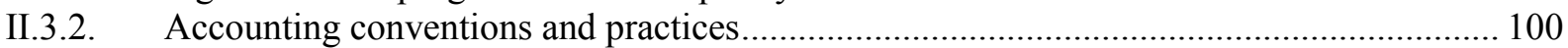

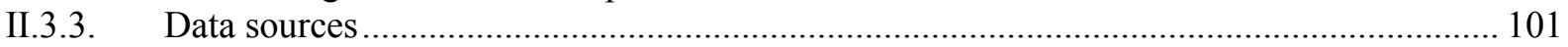

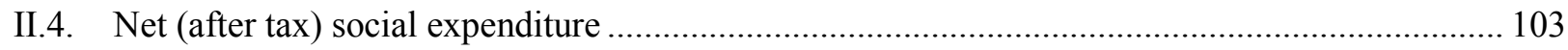

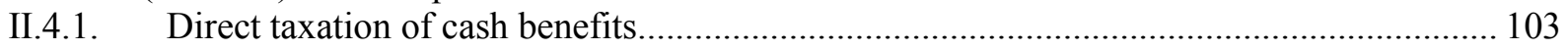

II.4.1.1. Methods and sources; administrative records, microsimulation and microdata ............. 105

Estimating Average Itemised Tax Rates through 'Microsimulation' ........................................... 105

II.4.2. Indirect taxation of consumption out of benefit income .................................................... 107

II.4.2.1. Methods and sources: national accounts and revenue statistics...................................... 107

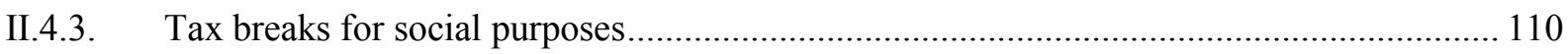

II.4.3.1. Methods and sources; the valuation of tax revenue forgone......................................... 110

II.4.3.2. Which tax breaks are included in the calculations and which are not? .......................... 112

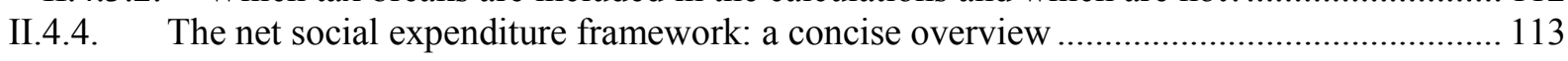

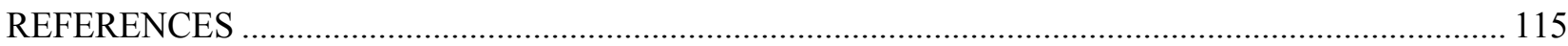

ANNEX II.1. DETAILED INFORMATION ON SOURCES OF TEH OECD SOCIAL EXPENDITURE

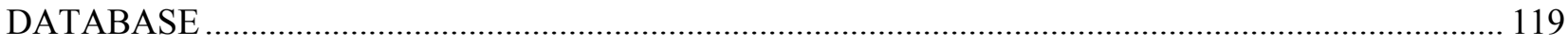

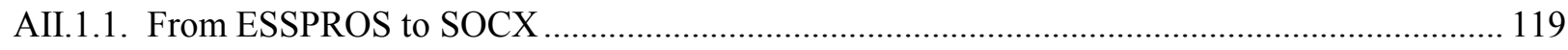

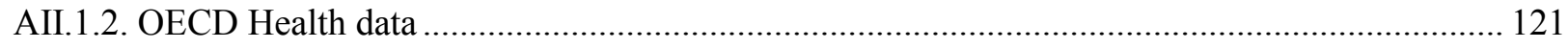

AII.1.3. OECD Labour Market Programmes database.......................................................................... 123

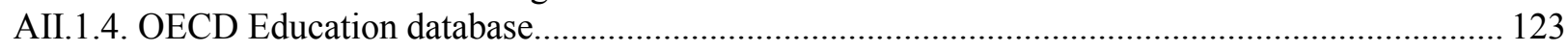

ANNEX II.2. HOW TO ACCESS SOCX ELECTRONICALLY? .................................................... 125 


\section{Tables}

Table I.1: In Anglophone countries income-testing plays an important role in social policy 19

Table I.2: Between 2007 and 2010 income support for the working-age population increased most in Estonia, Ireland, Spain, the United Kingdom and the United States..................................................... 21

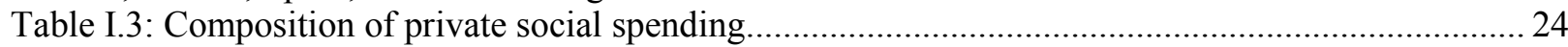

Table I.4: From gross public to net total social spending, 2007............................................................ 33

Table II.1: Categorisation of benefits with a social purpose _................................................................ 94

Table II.2: Structure of SOCX database for public and mandatory private programmes ....................... 96

Table II.3: Tax treatment of benefits differs across countries............................................................... 104

Table II.4: Average implicit indirect tax rates of consumption out of benefit income .......................... 109

Table II.5: Value of selected tax breaks for pensions, the United States, 2007 .................................. 111

Table II.6: From gross to net social expenditure: a concise overview …............................................. 114

\section{Boxes}

Box II.1: The relationship between OECD, Eurostat and ILO social accounting systems ..................... 89

Box II.2: Earnings and deferred wages; the treatment of pensions and severance payments in SOCX ... 91

Box II.3: Identifying and categorising benefits with a social purpose .............................................. 95

Box II.4: An example of calculating Average Itemised Tax rates .................................................... 106

\section{Charts}

Chart I.1: Social spending has increased in most OECD countries since 1980

Chart I.2: Growth in real social spending largely outpaced real GDP growth over the 1990-2007 period12 Chart I.3: Since 2009 real social spending has stabilized in most OECD countries and is falling in Greece, Hungary, Iceland, Ireland and Portugal.

Chart I.4: In an environment of economic growth, social spending often grows faster than GDP, but not

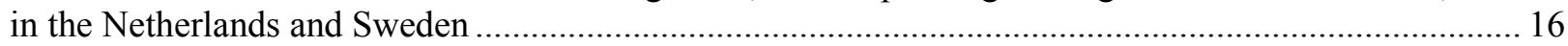
Chart I.5: On average OECD countries spend 7\% of GDP on pensions and 6\% on health services ........ 17 Chart I.6: The recent crisis had the largest effect on unemployment rates in Estonia and Spain.............. 20

Chart I.7: Gross private social spending has increased since 1990 ….................................................. 22

Chart I.8: A large tax burden on benefit income in Denmark and Sweden........................................... 27

Chart I.9: Indirect taxation is least important in Non-European OECD countries.................................. 28

Chart I.10: A high value of TBSPs in the United States, while they are virtually non-existent in

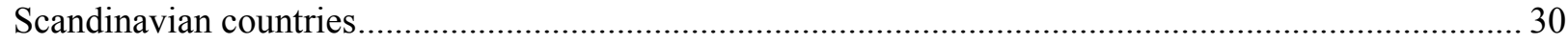

Chart I.11: Using net rather than gross indicators changes the ranking among countries in international comparison of social spending 


\title{
IS THE EUROPEAN WELFARE STATE REALLY MORE EXPENSIVE?
}

\author{
Indicators on social spending, 1980-2012; and, \\ a manual to the OECD Social Expenditure database (SOCX)
}

\section{Introduction}

1. The OECD Social Expenditure database (SOCX) was developed to analyse trends in social spending and its composition at the detailed social expenditure programme level. The detailed information in SOCX enables users to verify spending aggregates, regroup spending items for the analysis of selected social policy areas, and undertake cross-national analysis of trends in social policy reform. Detailed spending series are available up to 2007. For most countries, the series go back to 1980, but for countries that joined the OECD in the 1990s and 2000s data may only be available for shorter periods. This version of SOCX includes information on spending for 34 OECD countries, including Chile, Estonia, Israel and Slovenia which joined the OECD in 2010.

2. Detailed SOCX data is not available for the period after 2007. However, the public social expenditure series as in SOCX was extended to 2008, 2009 and 2010 using available information on national aggregates in the OECD Economic Outlook (OECD, 2011a) and the European Union's Annual macro-economic database (AMECO), or country responses to an OECD questionnaire on social policy experiences during the economic crisis. Based on projections in the OECD Economic Outlook and AMECO, public social spending aggregates were estimated for 2011 and 2012.

3. Over the years, SOCX has been developed in different dimensions, particularly in the areas of private social expenditure, spending on children in formal early care and education services, and net - after tax - indicators of social expenditure. The OECD Social Policy Division works closely with the OECD Centre for Tax Policy and Administration to continuously improve the quality of the net social expenditure indicators which are now available for 27 countries. Unfortunately, the necessary detail to obtain good estimates on the amount of tax levied on benefits only becomes available and can be collected about 2 to 3 years after the date. The next collection of SOCX data up to and including tax estimates for 2009 will be in late 2011.

4. This working paper is in two parts. Part I presents social spending indicators on trends and the composition of gross and net public and private social expenditure. Annex I.1 to this part of the paper provides additional information on the methodology and sources underlying the estimates of social spending that were made for recent years, whereas Annex I.2 provides detailed information per country and other background information on net social expenditure indicators. Part II presents the SOCX Manual, which defines the social protection domain; outlines categorisation and recording practices; and, discusses the methodology underlying net social spending indicators. Its Annexes present detailed information on sources used with respect to gross spending items in SOCX; and, on how to access SOCX electronically (see also www.oecd.org/els/social/expenditure). 


\section{PART I: INDICATORS ON SOCIAL SPENDING, 1980 - 2012}

\section{I.1. Summary and Main findings}

5. Part I discusses the main gross (before tax) public spending trends, both overall and by broad social policy area from 1980 to 2007. Aggregate social spending data is also presented for $2008-2010$ as well as estimates for 2011 and 2012. The composition of gross public and private social spending and the ways in which tax systems affect social expenditure totals are also examined. Tax effects are the result of direct taxation of public and private cash transfers, indirect taxation of consumption financed out of benefit income, and public support granted through tax breaks with a social purpose. Part I findings include:

- Since 1980 public social spending has increased by more than $20 \%$ across the OECD. Experiences differ across countries, but on average across the OECD, public social expenditure as a percent of GDP has increased from $15.6 \%$ in 1980 to $19.2 \%$ in 2007.

- Public expenditure on health and pensions are the largest social spending items. On average across the OECD, public spending on pensions was 7\% of GDP in 2007 and public expenditure on health amounted to $5.8 \%$. Public spending on income support for the working-age population (3.9\% of GDP) and on other social services (2.1\%) was comparatively limited.

- Public social spending-to-GDP-ratios initially increased in 2008 and 2009 in many OECD countries as the crisis unfolded in 2008/09 as social spending rose and GDP-growth slowed. On average, public social spending increased from $19.2 \%$ in 2007 to $22.5 \%$ in 2009 . In some countries, fiscal tightening and/or re-ignited economic activity have reduced social spending-toGDP ratios (including Greece, Iceland and Ireland), while in others spending-to-GDP ratios have stabilized. The public social spending-to-GDP ratio is projected to be around $22 \%$ on average across the OECD in 2012.

- At over $10 \%$ of GDP in 2007, private social spending was largest in the United States, which reflects the importance of private health spending. Pension benefits and/or mandatory employerprovided incapacity benefits are the other significant areas of private spending. At over 5\% of GDP in 2007, private social spending is also important in Canada, Iceland, the Netherlands, Switzerland and the United Kingdom.

- Direct and indirect taxation of benefit income and associated consumption is much more important in European OECD countries than in non-European member countries. At $5 \%$ of GDP or more in 2007, the amount of direct and indirect tax levied on public benefit income was highest in Denmark, Finland and Sweden.

- Tax breaks for social purposes (excluding pensions as there is no agreement on how to measure their real effect across countries) are generally least important in countries with relatively high direct tax levies. Tax breaks similar to cash benefits were worth around 1.0\% of GDP in Canada, France, Germany and Portugal. Tax breaks towards current private spending arrangements (health insurance) were largest in the United States at over 1\% of GDP in 2007.

- Net (after tax) public social expenditure is significantly below the levels suggested by gross public and private social expenditure indicators, except for Australia, Canada, Japan, Korea, Mexico, Turkey and the United States. This is because most countries levy significant direct and indirect taxes on social benefits and associated consumption.

- Accounting for both the tax system and the role of private social benefits reveals that total social spending as a share of GDP is similar in countries which are often thought to have very 
different levels of social spending. Total net social spending in Austria, Canada, Denmark, Finland, Italy, Japan, the Netherlands, Portugal, the United Kingdom and the United States only differ by a few percentage points of GDP.

\section{I.2. Trends in public social spending}

6. Since 1980, gross public social expenditure has increased from about $15.6 \%$ to $19.2 \%$ of GDP in 2007 on average across the 34 OECD countries (Chart I.1). Experiences differ across OECD countries, but on average, public social spending-to-GDP ratios increased most significantly in the early 1980s, early 1990s and, again in the beginning of this millennium. In between these decennial turning points spendingto-GDP ratios changed little; during the 1980s the average OECD public social spending-to-GDP ratio oscillated around $17 \%$ of GDP while during the 1990s it was generally just below $20 \%$ of GDP after the economic downturn in the early 1990s.

7. In most OECD countries, spending-to-GDP ratios in 2007 were well above 1980s levels, except for Ireland and the Netherlands in particular, where during the 1990s persistent economic growth, tightening of generosity of, and inflow into, disability benefits, and the privatisation of sick-pay led to a decline in the public social spending-to-GDP ratio by 4\% of GDP (Chart I.1). The most important increases in the public social expenditure-to-GDP ratios (by more than 4 percentage points) were recorded for Denmark, Finland, Ireland, Japan, Spain, Estonia, the United States and the United Kingdom. Other countries, such as Australia, Hungary, Israel, Switzerland and Poland recorded a much more modest increase in term of public social spending as a percent of GDP: around 1 percentage point or less than half the OECD average (for all years, see Annex I.1).

8. The two key drivers of increases in social spending period have been increased support for the (growing) retired population and health expenditure; population projections suggest further spending increases in these two areas in future (Adema and Ladaique, 2009). On average across OECD countries, public spending on old age increased from 5.1\% of GDP in 1980 to $6.4 \%$ in 2007. Similarly, public expenditure on health increased from $4.5 \%$ of GDP in 1980 to $5.8 \%$ in 2007. On average across the OECD (and the same holds for EU-21), spending on family benefits has increased by half a percentage point of GDP since 1990 (there was no significant change in the 1980s).

\section{I.3. Public social spending 2008-2012}

9. Chart I.1 shows that the economic crisis which started in 2008/09 has had an important effect on indicators of social spending. International comparisons of the magnitude of the welfare state are often measured by comparing public social expenditure-to-GDP ratios. Using available information on national aggregates to extend the SOCX series to 2008, 2009 and 2010 (Annex I.1) suggests that, on average across the OECD, the public social spending-to-GDP ratio increased from $19.2 \%$ in 2007 to $22.5 \%$ at peak in 2009. Public social spending-to-GDP ratios rose rapidly in 2009 and 2010. Projections suggest they will stabilize and sometimes decline in 2011 and $2012^{1}$ (Annex I.1). Nevertheless, levels remain higher than recorded prior to the economic crisis: on average across the OECD public social spending as a percent of GDP was 3 percentage points higher in 2010 than in 2007 (22.2\% compared with $19.2 \%$ in 2007).

$1 \quad$ Estimates on social spending-to-GDP ratios for 2011 and 2012 are based on indicators on social spending and GDP as in OECD (2011a), Economic Outlook which was released in May 2011. Revisions of GDP growth released in September 2011 suggest that the May 2011 estimates may have overestimated GDP growth by 1 percentage point on average across the G7 economies (in September 2011, there were no revised estimates social spending aggregates as in OECD, 2011a). This suggests that spending-to-GDP ratios for 2011 may have been overestimated by 0.2 percentage points. 


\section{Chart I.1: Social spending has increased in most OECD countries since 1980}

Public social spending aggregates based on detailed data for 1980-2007, national aggregates for 2008-2010 and estimates for 2011 and 2012, in percentage of GDP
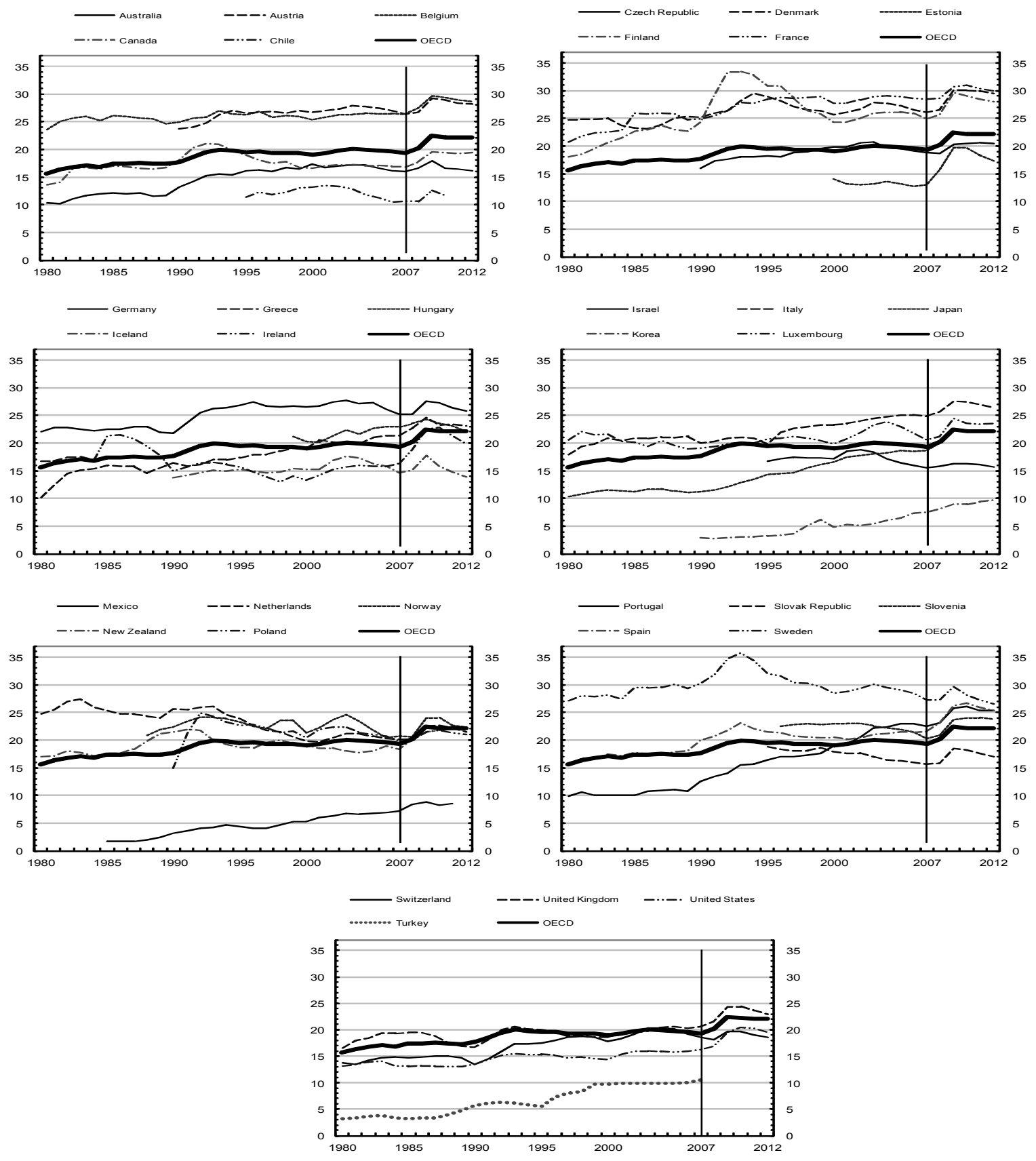

Note: 2000-2004 data for Turkey are linearly estimated between 1999 and 2005 figures. Data are available for 34 OECD countries from 2000. Data prior to 2000 have been interpolated backwards from an unweighted OECD average based from 23 OECD countries. For information on the methodology for projections, please see Annex I.1. Information on national spending aggregates beyond 2009 and 2008 is not available for Japan and Turkey respectively. Because of the natural disaster in Chile in 2010, no estimate was made for 2011-12.

Source: OECD Social Expenditure Database (SOCX, www.oecd.org/els/social/expenditure). 
10. Public social expenditure-to-GDP ratios increased in all OECD countries during the recent economic downturn. The largest increases took place in Estonia, Ireland and Spain, and were more limited in Israel, Switzerland, and Hungary (Chart I.1 and Annex I.1).

\section{I.3.1. Disentangling spending and GDP trends}

11. To facilitate international comparison, indicators on social spending are usually related to GDP. However, trends in spending and economic growth need to be separated in order to assess the evolution of social expenditures. Chart I.2 shows that over the 1990-2007 period, public social spending in real terms by and large grew faster than GDP across the OECD area (real spending growth tailed off in Australia after 2004). In Japan, real spending growth has outpaced sluggish real GDP-growth since 1990, so much so that the public social expenditure-to-GDP ratio increased from $11.3 \%$ in 1990 to $18.7 \%$ in 2007 (Chart I.2).

12. Diverging real spending and GDP trends also underline the rapid increase in social spending-toGDP ratios in the late 2000s. With the economic downturn, GDP growth slowed in most countries. Nevertheless, real GDP in 2012 is expected to be above 2007-levels except for the Czech Republic, Greece, Hungary, Ireland, Italy, Japan and the United Kingdom (Chart I.3).

13. Between 2007 and 2010, national aggregates suggest that social spending in real terms increased by $10 \%$ or more in most OECD countries, and this increase was $20 \%$ or more in Chile, Estonia, Korea, Luxembourg, Spain and the United States. Overall, real spending trends are estimated to be relatively flat from 2009/10 onwards in most countries. Pronounced falls in real social spending after 2008/09 as a result of fiscal tightening are estimated for Greece, Hungary, Iceland, Ireland and Portugal.

\section{Chart I.2: Growth in real social spending largely outpaced real GDP growth over the 1990-2007 period}

Real public social expenditure and real GDP from 1990 to 2007 , Index1990=100
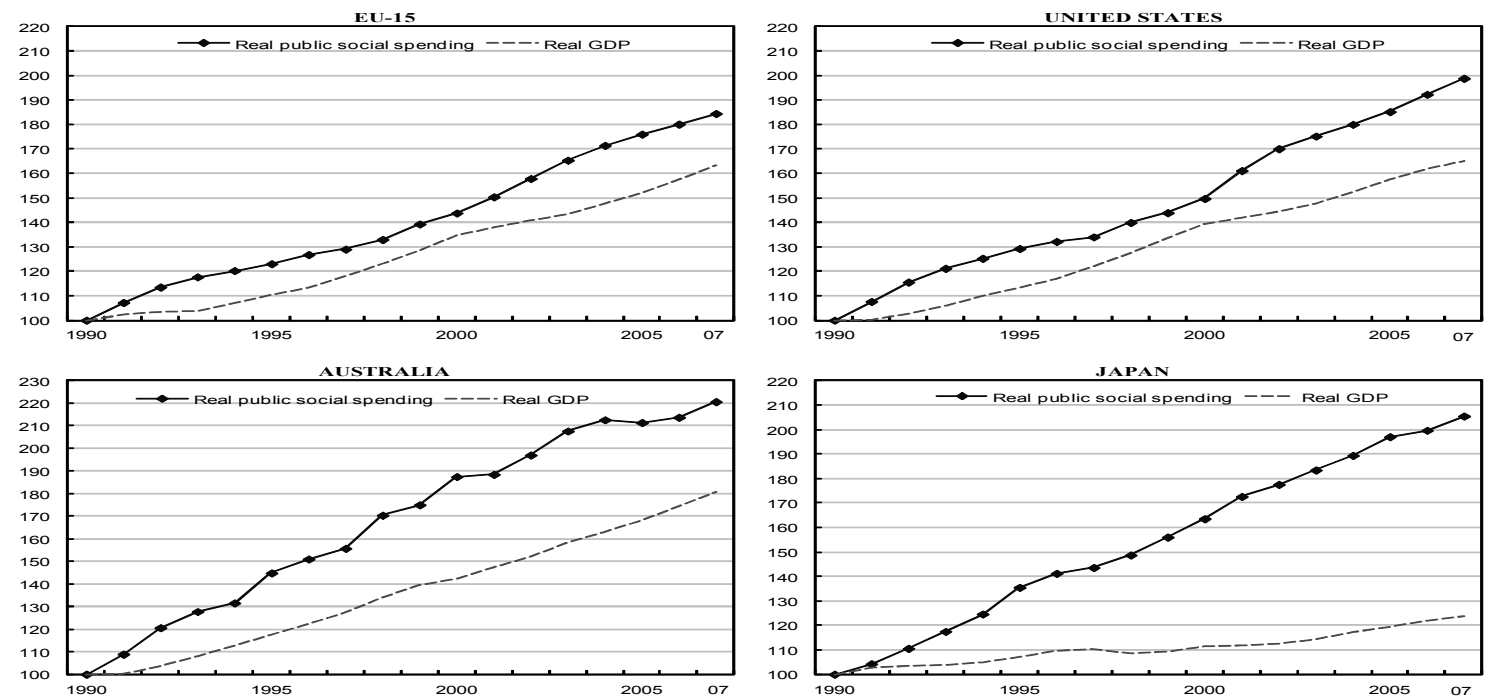

Notes: Information on Superannuation benefits paid to former civil servants in Australia became available in 1995, which contributed to the rapid increase of real public social spending at the time. Rapid growth of Australian social spending in 2000 reflects policy changes: in particular the one-off payment of the "Aged Persons Savings Bonus" and increased spending on family support through the "Family Tax Benefit".

The Japanese health accounts record data on basis of the principles of the OECD System of Health Accounts from 1995 onwards; the break in the series contributes to seemingly accelerate spending growth in Japan for 1995.

Source: OECD Social Expenditure Database (SOCX, www.oecd.org/els/social/expenditure). 
Chart I.3: Since 2009 real social spending has stabilized in most OECD countries and is falling in Greece, Hungary, Iceland, Ireland and Portugal.

Estimates of real public social spending and real GDP (Index 2007=100) and public social spending in percentage of GDP (right scale), 2007-2012
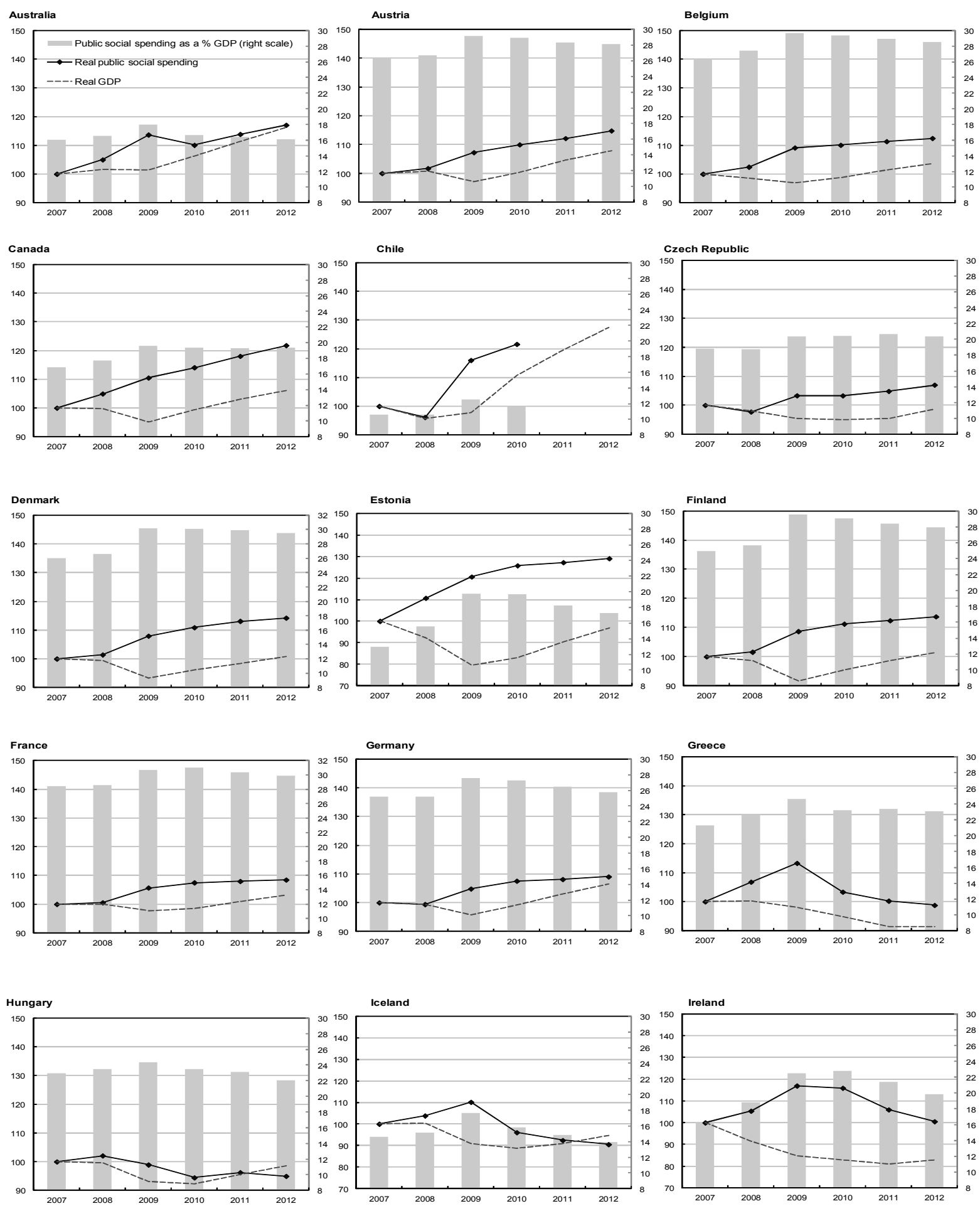
Chart I.3: Since 2009 real social spending has stabilized in most OECD countries and is falling in Greece, Iceland, Ireland and Portugal (cont.)

Estimates of real public social spending and real GDP (Index 2007=100) and public social spending in percentage of GDP (right scale), 2007-2012
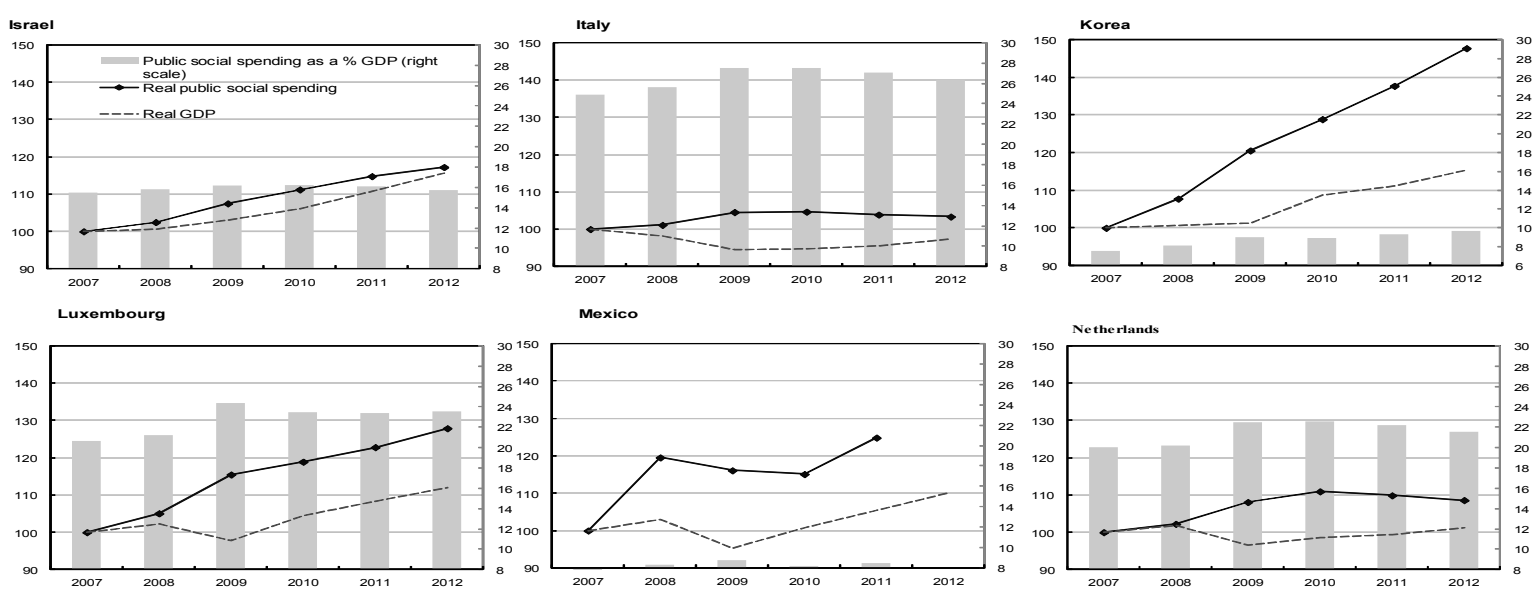

Mexico
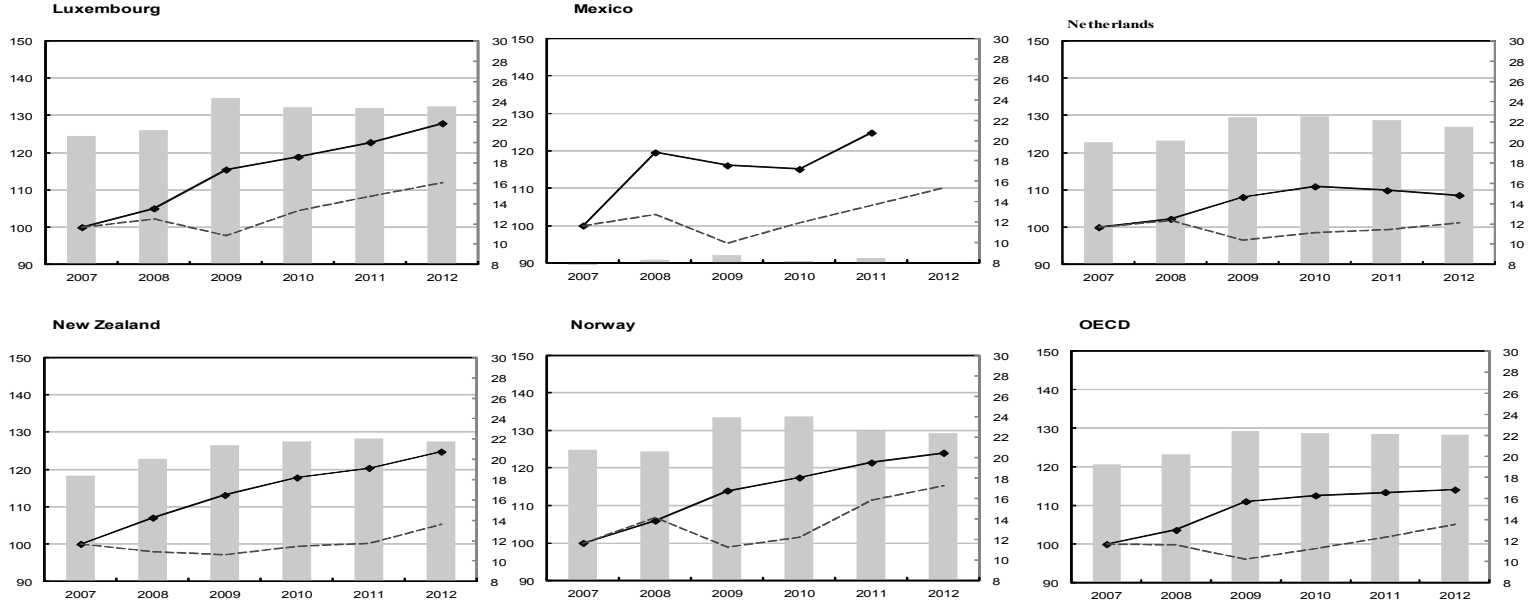

OECD

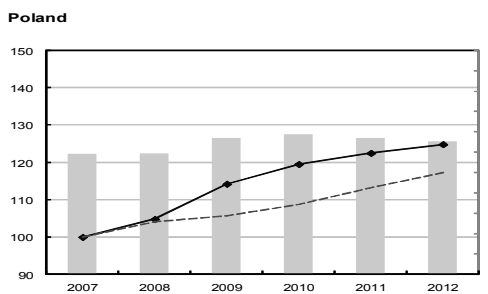

Portugal

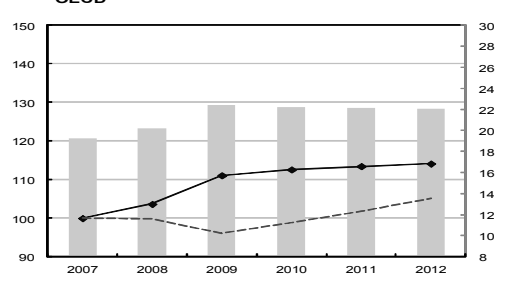

Slovak Republic
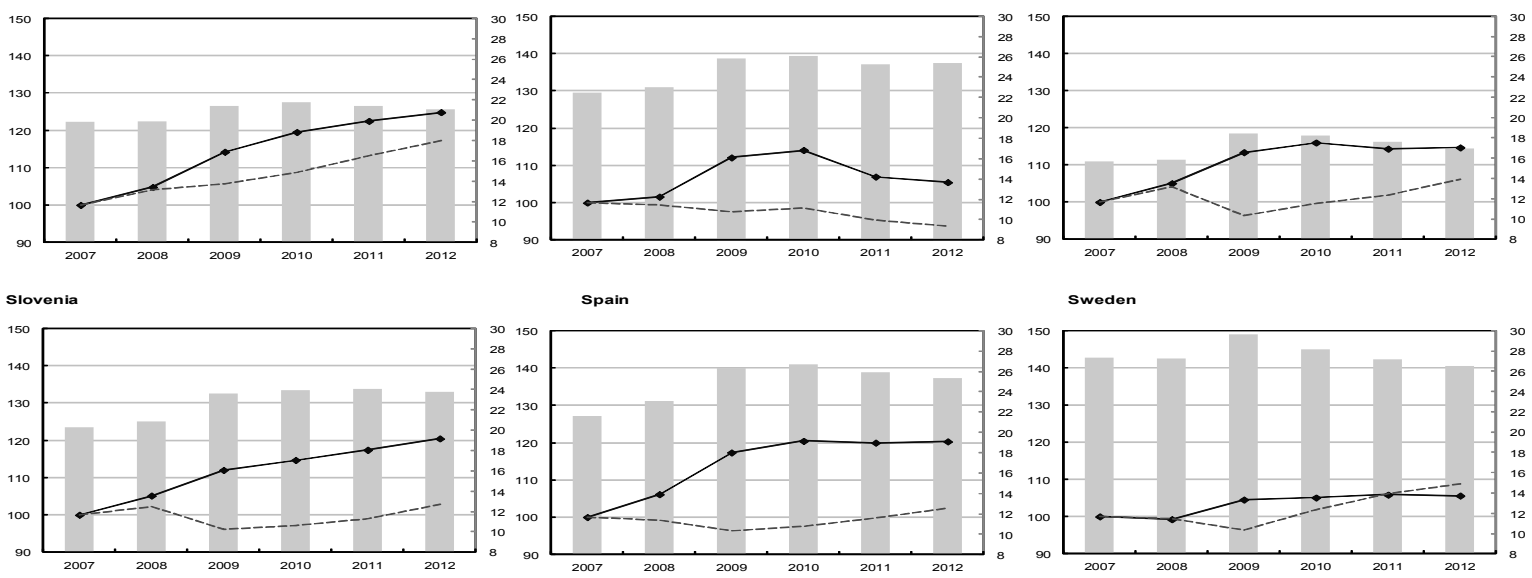

Sweden
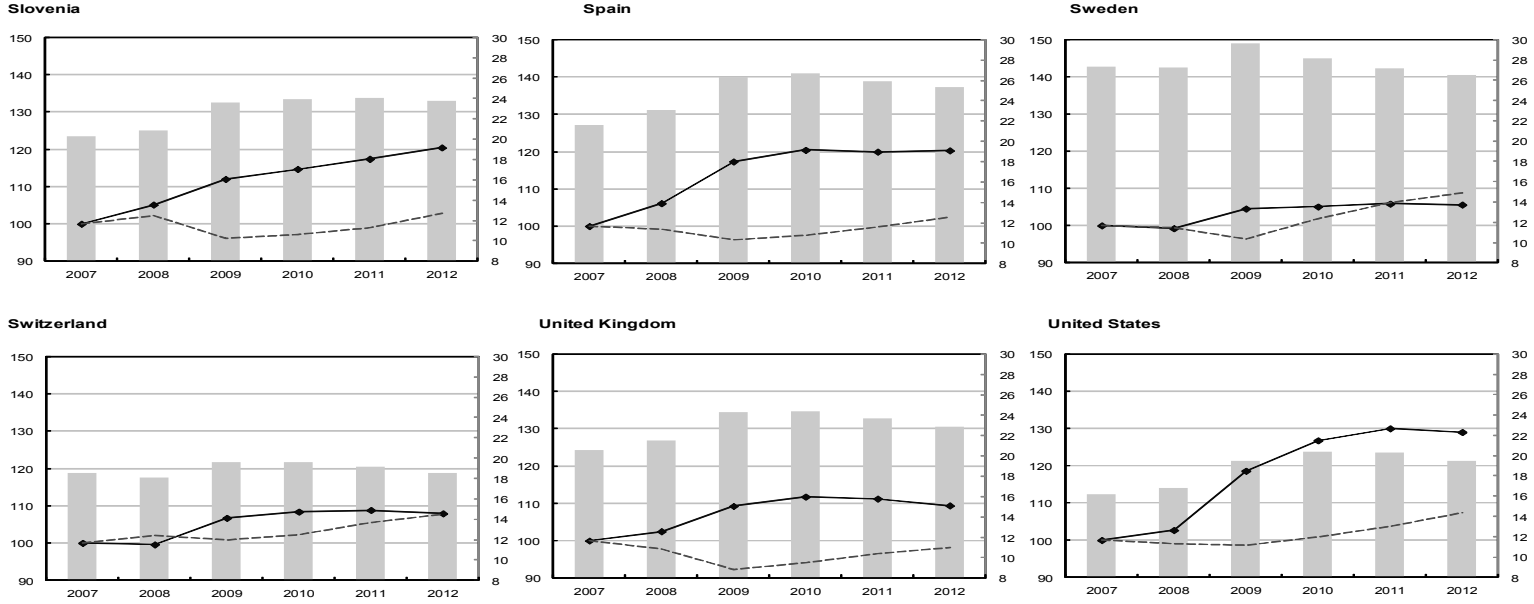

United Kingdom
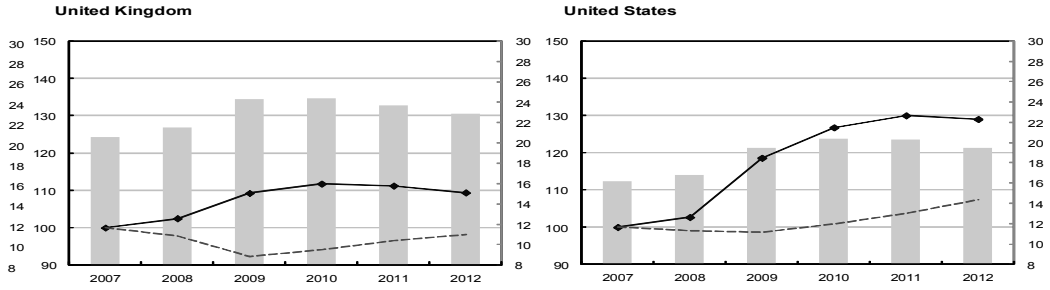

Note: see Annex I.1 for information on data and methodology underlying the estimates.

Source: OECD Social Expenditure Database (SOCX, www.oecd.org/els/social/expenditure). 


\section{I.3.2. Comparing social spending to "trend GDP"}

14. Charts I.1 and I.3 showed that international comparisons of spending-to-GDP ratios not only reflect differences in spending levels but also differences in levels of economic activity. The impact of the economic crisis differed across OECD countries in terms of timing and intensity. International comparisons of spending-to-GDP-ratios are more generally affected by the fact that economies are not all at the same stage of the cycle at the same time. Therefore, an adjustment is needed to capture the automatic budget effects resulting from deviations from structural GDP.

15. Chart I.4 presents real GDP data and public social expenditure ratios with actual GDP and "trend GDP" for those OECD countries where data over the complete 1980-2012 period is available. Trend GDP has been calculated applying a Hodrick-Prescott filter (smoothing-factor 1000) on GDP data for the period 1980-2012, (for more information on definitions, see OECD System of Composite leading indicators, http://www.oecd.org/dataoecd/26/39/41629509.pdf). This non-linear "trend GDP" does not properly adjust for GDP-fluctuations with the cycle and therefore is not equivalent to structural GDP. However, deviations from "trend GDP" approximate deviations from structural GDP to a certain extent and as such can be used as an illustration.

16. When social expenditure grows in line with GDP the ratio will remain stable over time as, for example, in Canada for the years 2002-2007. But such stability is rare. Between 1980 and 2008/09 GDP grew continuously in most countries for which the full spending times series is available. In France, Greece, Italy, Portugal and Switzerland (and to a lesser extent the United States) this was associated with rising ratios of social spending to GDP, while that ratio fell in the Netherlands and Sweden (after the crisis in the early 1990s).

17. As expected, economic crises bring about significant deviations between social spending as related to trend and actual GDP. The Finnish and Swedish experiences in the early 1990s and most OECD countries for recent years show this clearly. The stage of the cycle can be very different across countries For example, in the early 1990s Belgian GDP continued to grow, in contrast to what happened in Finland (Chart I.4). The variation in timing and size of cyclical fluctuations can diminish the "power" of crossnational comparisons of social-spending-to-GDP ratios. 
Chart I.4: In an environment of economic growth, social spending often grows faster than GDP, but not in the Netherlands and Sweden

Projected public social spending as a \% GDP and as a \% "trend GDP" and real GDP, 1980-2012
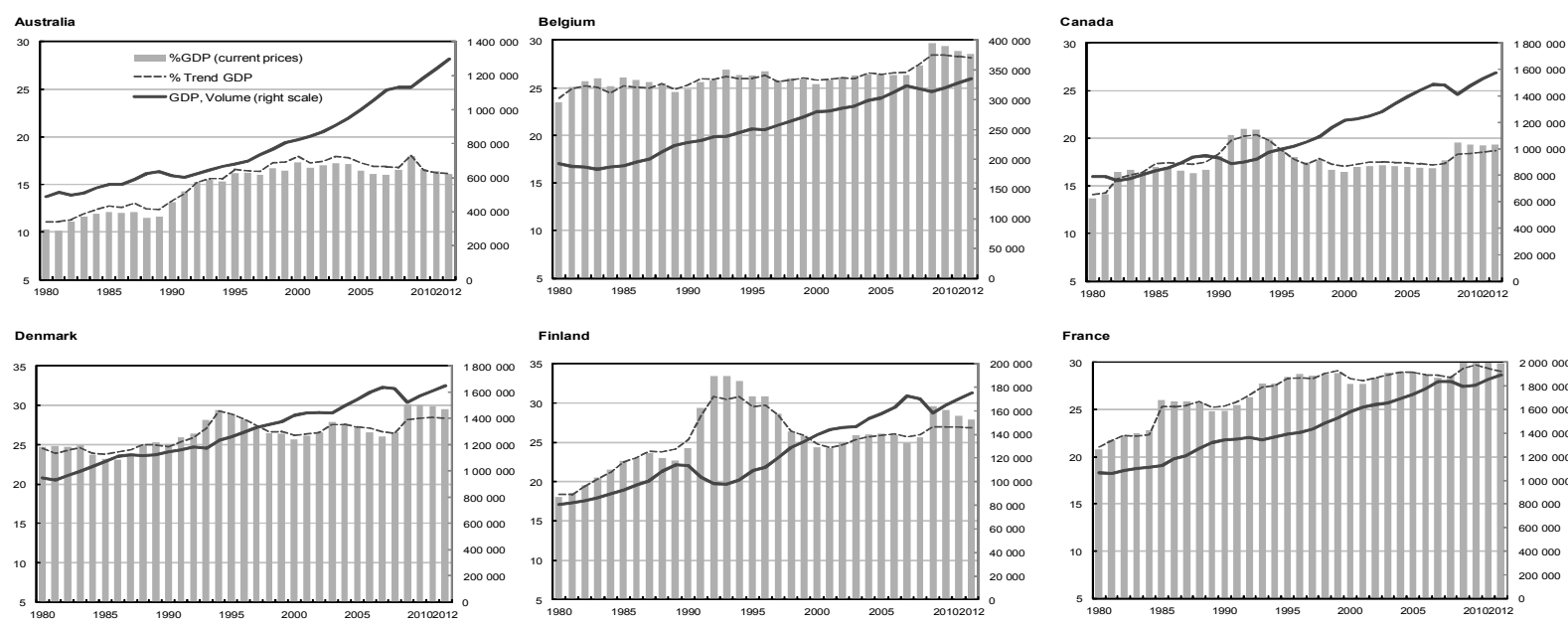

Finland
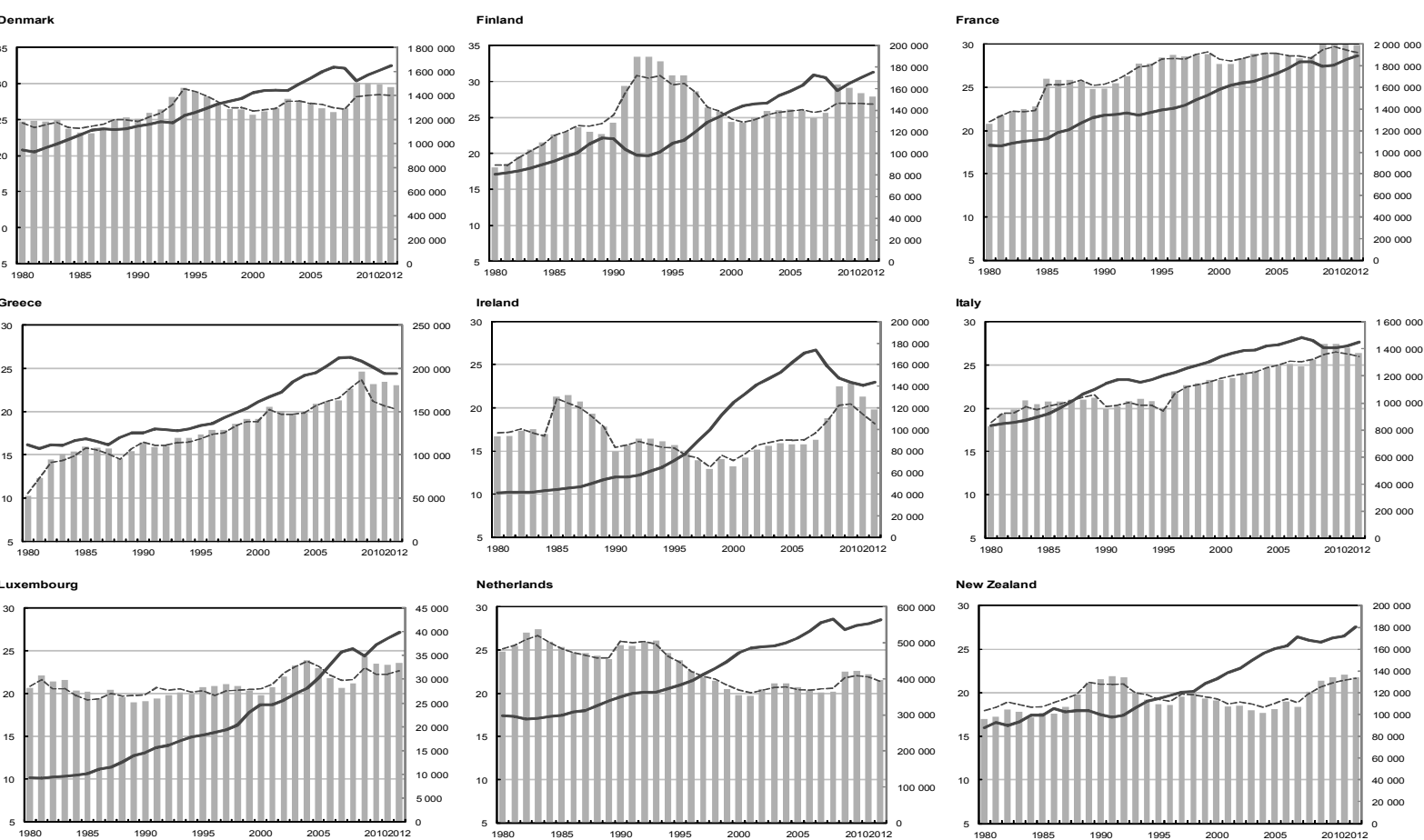

New Zealand

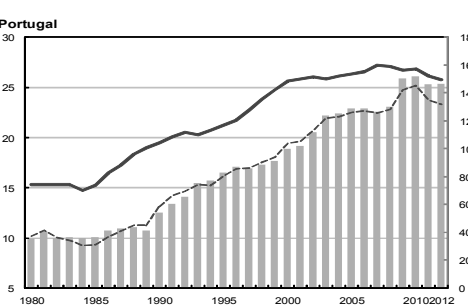

Spain
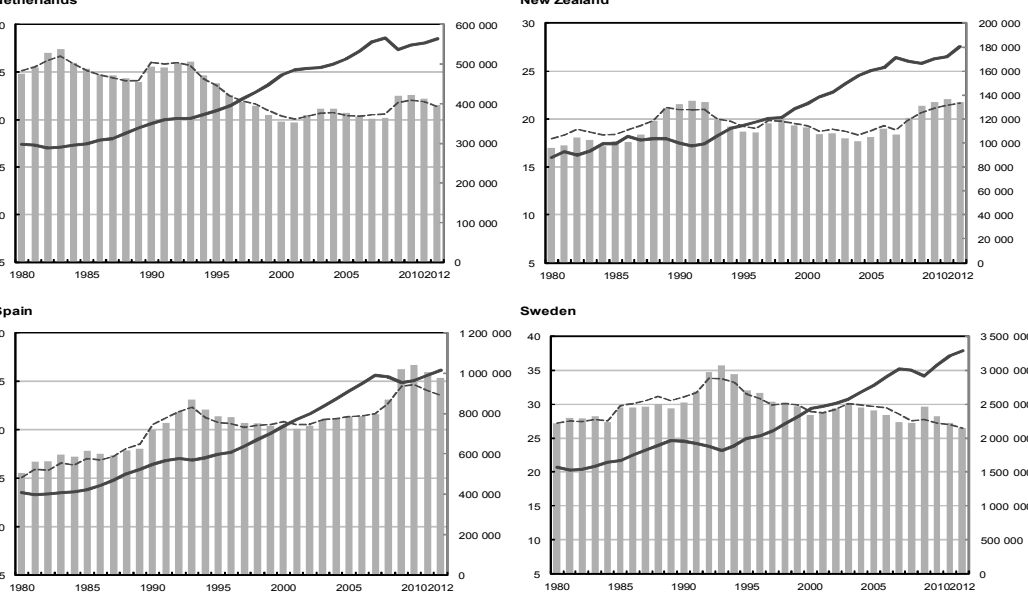

Sweden
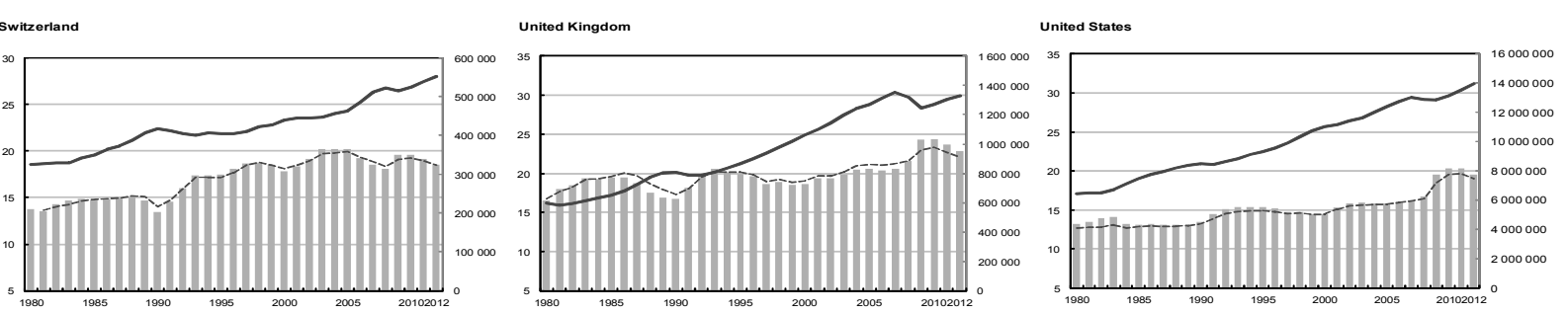

Note: see Annex I.1 for information on data and methodology underlying the estimates.

Source: OECD Social Expenditure Database (SOCX, www.oecd.org/els/social/expenditure), OECD Economic Outlook 89A. 


\section{I.4. The composition of public social spending in 2007}

18. In 2007, gross public social expenditure was 19.2\% of GDP on average across OECD countries, with spending on cash benefits as on services (Chart 1.5). Cross-country differences in public social spending are wide, ranging from 7\% of GDP in Mexico and Korea to just over 28\% in France and 27\% in Sweden. The largest category of public social spending concerns old-age and survivors' pensions; on average across the OECD, they account for 7\% of GDP, excluding pension payments through autonomous programmes to former civil servants since these are categorised as private spending in line with the System of National Accounts (SNA). However, public spending on old-age and survivor pensions account for more than 14\% in Italy, above 12\% of GDP in Austria and France, but less than 4\% in Australia, Iceland, Ireland, Korea and Mexico. On average across the OECD, income transfers to the working-age population amounted to just over $4 \%$ of GDP.

\section{Chart I.5: On average OECD countries spend $7 \%$ of GDP on pensions and $6 \%$ on health services}

Public social expenditure by broad social policy area in percentage of GDP, 2007

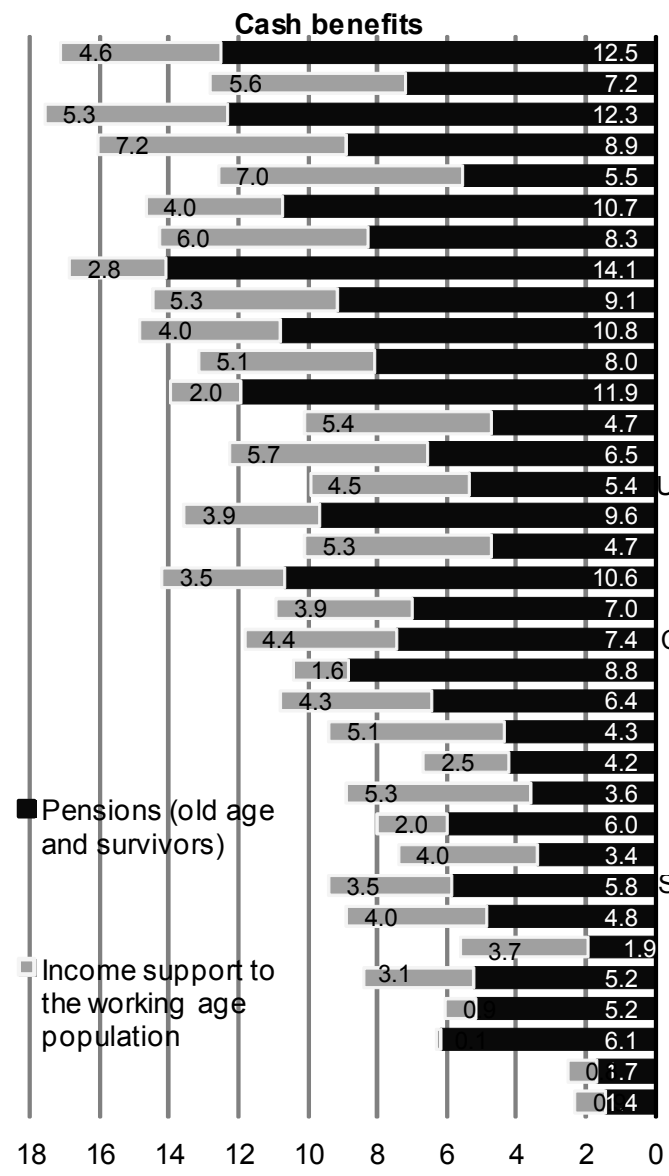

France (28.4)

Sweden (27.3)

Austria (26.4)

Belgium (26.3)

Denmark (26)

Germany (25.2)

Finland (24.9)

Italy (24.9)

Hungary (22.9)

Portugal (22.5)

Spain (21.6)

Greece (21.3)

Norway (20.8)

Luxembourg (20.6)

United Kingdom (20.5)

Slovenia (20.3)

Netherlands (20.1)

Poland (19.8)

OECD-34 (19.2)

Czech Republic (18.8) Japan (18.7)

Switzerland (18.5)

New Zealand (18.4)

Canada (16.9)

Ireland (16.3)

United States (16.2)

Australia (16)

ovak Republic (15.7)

Israel (15.5)

Iceland (14.6)

Estonia (13)

Chile (10.6)

Turkey (10.5)

Korea (7.6)

Mexico (7.2)

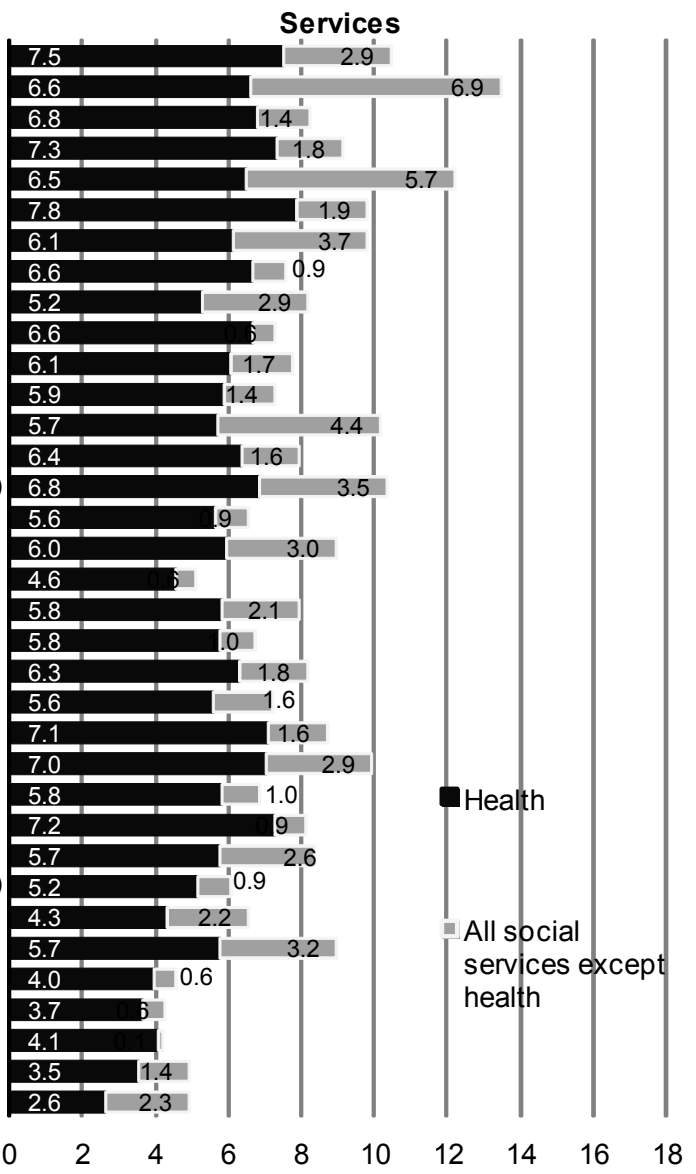

Note: Countries are ranked by decreasing order of public social expenditure as a percentage of GDP. Spending on Active Labour Market Programs (ALMPs) cannot be split by cash/services breakdown; they are however included in the total public spending (shown in brackets).

Source: OECD Social Expenditure Database (SOCX, www.oecd.org/els/social/expenditure). 
19. Public expenditure on health services amounted to less than $6 \%$ of GDP on average in 2007 while spending on other social services (e.g, childcare, home help) was just over $2 \%$ of GDP. The latter exceeded 5\% of GDP only in Denmark and Sweden, where the public role in providing services to the elderly, the disabled and families is extensive. In southern and eastern European countries, Chile and the United States, other social services accounted for about $1 \%$ of GDP due to the greater reliance on private and informal care.

20. Since previous versions of SOCX, considerable efforts have been made to improve reporting in SOCX of childcare and early education services. Spending figures are now more consistent across countries. All available data on public financial support for families with children participating in both formal day-care services (i.e., crèches, day-care centres and family day-care for children under 3 ) and preschool institutions (including kindergartens and day-care centres for children aged from 3 to 5 inclusive). Improved information in SOCX on publicly supported childcare and early education services show that on average public spending in this regard was just below $0.6 \%$ of GDP in 2007, with considerable crosscountry variation: from about $0.2 \%$ of GDP in Switzerland to almost $1.3 \%$ of GDP in Denmark (OECD Family database - Indicator PF3.1 - www.oecd.org/els/social/family/database). ${ }^{2}$

21. Most OECD countries pay income support to households which do not have sufficient other resources to support themselves, but the extent to which countries use income-tested programmes varies across countries. By selecting relevant programmes from country data files, SOCX facilitates identifying income-tested spending items. Table I.1 shows such spending for 2007, by including spending on "other social policy areas", income-tested spending on the unemployed, income-tested support payments to elderly and disabled, and other income-tested payments including family cash transfers (see note to Table I.1).

22. In 2007, spending on income-tested social programmes accounted on average for $2.0 \%$ of GDP, which corresponds to $11 \%$ of public social spending or $21 \%$ of public social spending on cash transfers. In countries that have an insurance-based support system (for example, Scandinavian countries, Belgium, France and Germany, Japan and Luxembourg), the role for income-tested programmes is usually limited and aimed primarily at those who have exhausted their unemployment insurance entitlements and are eligible for unemployment assistance or social assistance; spending is less than $5 \%$ of all public social spending. But in countries such as Australia, Canada, Iceland and the United Kingdom, the role of incometested programmes is considerably larger. Overall income-tested social programmes have seen an increase over the last years and this has also been reinforced in the context of the economic crisis in order to ensure support for the least well-off.

$2 \quad$ A side effect of making SOCX more consistent is that some spending is included both in SOCX and in OECD Statistics on education spending. If for some reasons it is desirable to sum the two data series, an adjustment is now required, see Annex AII.1.4. 
DELSA/ELSA/WD/SEM(2011)9

Table I.1: In Anglophone countries income-testing plays an important role in social policy

Public social expenditure on income-tested programmes, 2007

\begin{tabular}{|c|c|c|c|c|}
\hline & Millions, national currency & $\%$ GDP & \% sOCX public & $\%$ socX public in cash \\
\hline Australia & 66125 & 5.6 & 34.9 & 75.9 \\
\hline Austria & 5129 & 1.9 & 7.1 & 10.7 \\
\hline Belgium & 4538 & 1.4 & 5.1 & 8.5 \\
\hline Canada & 56412 & 3.6 & 21.6 & 54.8 \\
\hline Chile & 316844 & 0.4 & 3.5 & 6.1 \\
\hline Czech Republic & 23853 & 0.7 & 3.6 & 5.7 \\
\hline Denmark & 27587 & 1.6 & 6.2 & 13.0 \\
\hline Estonia & 236 & 0.1 & 0.7 & 1.1 \\
\hline Finland & 2493 & 1.4 & 5.6 & 9.7 \\
\hline France & 77797 & 4.1 & 14.5 & 24.1 \\
\hline Germany & 80216 & 3.3 & 13.1 & 22.5 \\
\hline Greece & 4952 & 2.2 & 10.3 & 15.8 \\
\hline Hungary & 388491 & 1.5 & 6.7 & 10.6 \\
\hline Iceland & 51899 & 4.0 & 27.2 & 70.9 \\
\hline Ireland & 8128 & 4.3 & 26.3 & 48.4 \\
\hline Israel & 6208 & 0.9 & 5.8 & 10.2 \\
\hline Italy & 19076 & 1.2 & 5.0 & 7.3 \\
\hline Japan & 2842844 & 0.6 & 3.0 & 5.3 \\
\hline Korea & 7547338 & 0.8 & 10.2 & 30.7 \\
\hline Luxembourg & 213 & 0.6 & 2.7 & 4.6 \\
\hline Mexico & 94086 & 0.8 & 11.7 & 36.9 \\
\hline Netherlands & 20795 & 3.6 & 18.1 & 36.1 \\
\hline New Zealand & 5990 & 3.3 & 18.0 & 35.3 \\
\hline Norway & 32341 & 1.4 & 6.8 & 14.1 \\
\hline Poland & 10770 & 0.9 & 4.6 & 6.5 \\
\hline Portugal & 4486 & 2.7 & 11.8 & 18.0 \\
\hline Slovak Republic & 590 & 1.0 & 6.1 & 10.2 \\
\hline Slovenia & 669 & 1.9 & 9.5 & 14.3 \\
\hline Spain & 27871 & 2.6 & 12.3 & 20.2 \\
\hline Sweden & 33886 & 1.1 & 4.0 & 8.5 \\
\hline Switzerland & 8700 & 1.7 & 9.0 & 15.5 \\
\hline Turkey & 355 & 0.3 & 3.5 & 5.1 \\
\hline United Kingdom & 70096 & 5.0 & 24.1 & 50.0 \\
\hline United States & 167949 & 1.2 & 7.5 & 15.2 \\
\hline OECD average & - & 2.0 & 10.6 & 21.2 \\
\hline
\end{tabular}

Note: The following income-tested spending items are included: spending on "other contingencies - other social policy areas" as in the OECD Social Expenditure Database (SOCX), income-tested spending on the unemployed (e.g., unemployment assistance payments for Germany), income-tested support payments to elderly and disabled (e.g., Belgium and the United Kingdom), other income-tested payments (family cash transfers) but do not include specific housing subsidies, spending on Active Labour Market Policies, or income-tested medical support. Data for Turkey refer to 1999.

Source: OECD Social Expenditure database, www.oecd.org/els/social/expenditure.

\section{I.4.1. Trends in social expenditures 2007-2012 by broad social policy area}

23. In addition to extending SOCX estimates on aggregate public social spending, estimates were also made for spending along four broad public social spending groupings: pensions, income support to people of working age, health and other social services.

\section{Pensions}

24. Public spending on pensions includes public old age and survivors' cash benefits. These payments reflect long-term entitlements and financial commitments and are driven by demographic trends. They therefore tend to be far less cyclical than social spending on the working-age population (see below). With population ageing and the maturing of pension systems, public pension spending generally increases 
over the 2007-2012 period. This is particularly important in, for example, Korea where the pension system is now maturing. Many OECD countries are reforming their pension systems to limit spending and ensure long-term financial sustainability of pension systems (OECD, 2011b); recent reforms in the Czech Republic and Poland, for example, have contributed to significant declines in public pension spending as a share of GDP.

Income support to the working-age population

25. Spending on unemployment compensation fluctuates with the economic cycle. SOCX shows it peaked at $1.6 \%$ of GDP in 1993, but in 2007 it was at the same level as in the early $1980 \mathrm{~s}$ : at $0.7 \%$ of GDP. The economic crisis led to an increase in unemployment rates in all OECD countries, except Germany (partly due to the effect of short-term work programmes). Chart I.6 shows that by July 2010 "harmonised" unemployment rates exceeded 13\% in Ireland, the Slovak Republic, Estonia and Spain. The increase in unemployment led to increased spending on unemployment, social assistance benefits and sometimes other income support benefits to the working-age populations. OECD (2011c) shows that spending on unemployment compensation and Active Labour Market Programmes (ALMPs) rose considerably between 2007 and 2009. On average across the OECD, unemployment compensation (OECD Labour Market Programme Database, (2011c)): Out of work income maintenance and support) increased from $0.6 \%$ in 2007 to more than $1 \%$ of GDP in 2009 while spending on ALMPs increased from $0.50 \%$ to $0.62 \%$. The increases in public social spending for unemployed compensation increased significantly in Estonia, Greece, Ireland, Italy, Spain, the United Kingdom and the United States.

\section{Chart I.6: The recent crisis had the largest effect on unemployment rates in Estonia and Spain}

\section{OECD harmonised unemployment rates, in percentage of labour force, December 2007 to July 2010}

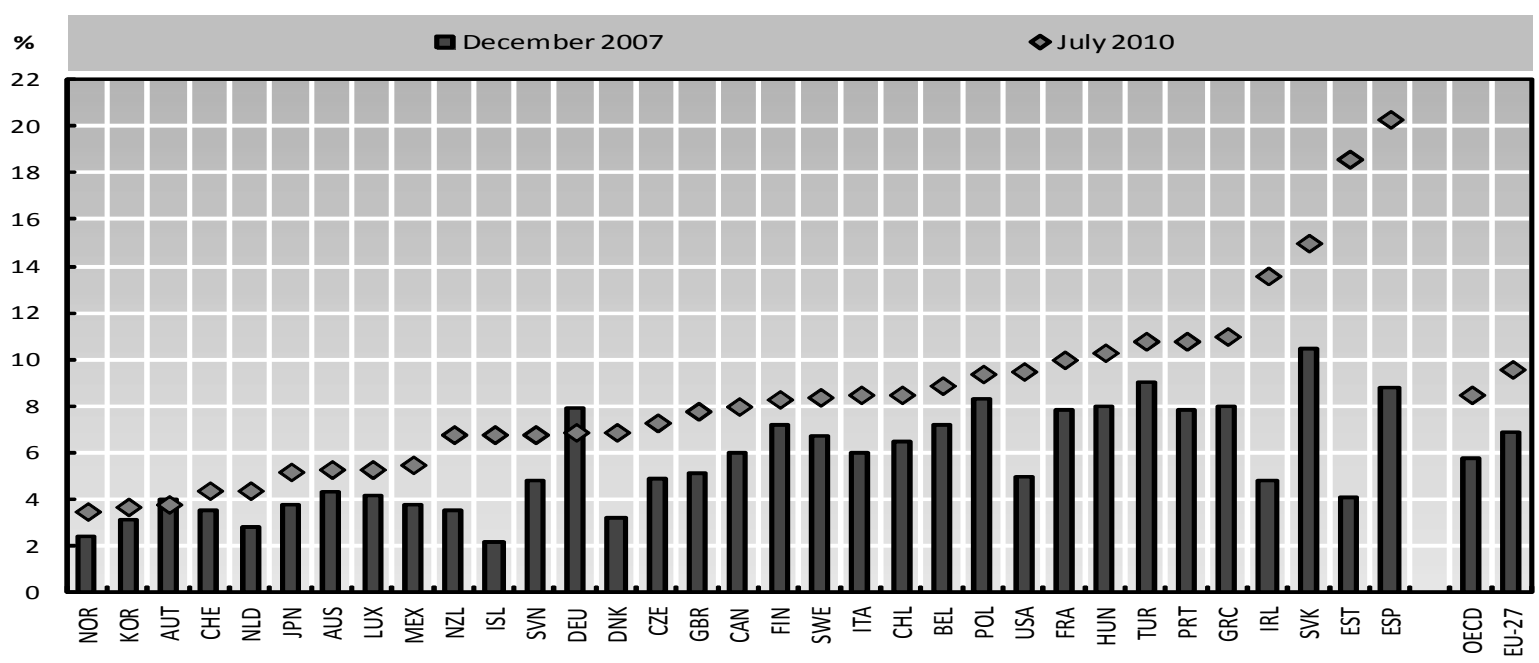

Note: All data are seasonally adjusted. March 2010 for Greece; April 2010 for Turkey; May 2010 for the United Kingdom; June 2010 for Chile, Estonia, Mexico, Norway and the Netherlands; and 2010 Q2 for Iceland, New Zealand and Switzerland (OECD harmonised unemployment rate data are not available on a monthly basis for the last three of these countries).

Source: OECD Labour Force Statistics database (www.oecd.org/employment).

26. Overall, for social spending on income support for the working-age population, at over 1 percentage point in GDP, increases in spending between 2007 and 2010 were most pronounced in Estonia, Ireland, Iceland, Spain, the United Kingdom and the United States. 
27. In response to the crisis many OECD countries initially extended social support to job-losers (e.g, as in the United States) or provided income support associated with shortened working hours (e.g., the Netherlands). However, many countries are now introducing reforms to reduce fiscal deficits and public debt. For example, Greece, Ireland, Spain and the United Kingdom, are among the countries that have made cuts in social benefits and tightened eligibility criteria (OECD, 2011d).

\section{Social and Health Services}

28. Expenditures on social and health services are generally much less cyclical than cash spending and it generally takes more time to adjust outlays on these items. Public health expenditures are a case in point, and their upward trend is also related to ageing populations. Public expenditure on health as a percent of GDP is expected to stay relatively stable over the 2007-12 period. Nevertheless, important increases were recorded in a few countries, including Canada, Chile, New Zealand, and the United States (Table I.2). In addition to the ageing of the population, determinants of health spending growth include also rising national income, relative medical prices and technological progress (OECD, 2010a).

Table I.2: Between 2007 and 2010 income support for the working-age population increased most in Estonia, Ireland, Spain, the United Kingdom and the United States

Composition of public social expenditure, in percentage of GDP, by broad category, 2007, 2010-2012 (estimates)

\begin{tabular}{|c|c|c|c|c|c|c|c|c|c|c|c|c|c|c|c|}
\hline & & & 2007 & & & & & 2010 & & & & & 2012 & & \\
\hline & $\begin{array}{l}\text { Public social } \\
\text { exp } \% \text { GDP }\end{array}$ & f which: & & & & $\begin{array}{l}\text { Public social } \\
\text { exp \% GDP }\end{array}$ & of which: & & & & $\begin{array}{l}\text { Public social } \\
\text { exp \% GDP }\end{array}$ & of which: & & & \\
\hline & & Pension & $\begin{array}{l}\text { Income support to } \\
\text { the working age }\end{array}$ & Health & $\begin{array}{c}\text { Other } \\
\text { services }\end{array}$ & & Pension & $\begin{array}{c}\text { Income } \\
\text { support to the } \\
\text { working age }\end{array}$ & Health & $\begin{array}{c}\text { Other } \\
\text { services }\end{array}$ & & Pension & $\begin{array}{l}\text { Income } \\
\text { support to the } \\
\text { working age }\end{array}$ & Health & $\begin{array}{l}\text { Other } \\
\text { services }\end{array}$ \\
\hline Australia & 16.0 & 3.4 & 4.0 & 5.7 & 2.9 & 16.6 & 3.4 & 4.1 & 6.1 & 3.0 & 16.1 & 3.4 & 3.8 & 6.2 & 2.7 \\
\hline Austria & 26.4 & 12.3 & 5.3 & 6.8 & 2.1 & 28.9 & 12.2 & 5.8 & 6.9 & 4.0 & 28.1 & 12.2 & 5.8 & 7.0 & 3.1 \\
\hline Belgium & 26.3 & 8.9 & 7.2 & 7.3 & 3.0 & 29.4 & 9.1 & 8.0 & 7.4 & 4.9 & 28.6 & 9.3 & 7.7 & 7.5 & 4.0 \\
\hline Canada & 16.9 & 4.2 & 2.5 & 7.0 & 3.2 & 19.3 & 4.6 & 2.8 & 8.4 & 3.6 & 19.3 & 4.7 & 2.7 & 8.7 & 3.2 \\
\hline Chile & 10.6 & 5.2 & 0.9 & 3.7 & 0.9 & 11.6 & 5.2 & 1.0 & 4.3 & 1.2 & .. & .. & .. & .. & .. \\
\hline Czech Republic & 18.8 & 7.4 & 4.4 & 5.8 & 1.2 & 20.4 & 6.8 & 4.7 & 5.9 & 3.0 & 20.4 & 6.7 & 4.7 & 6.1 & 3.0 \\
\hline Denmark & 26.0 & 5.5 & 7.0 & 6.5 & 7.0 & 30.1 & 5.7 & 8.0 & 6.6 & 9.7 & 29.5 & 5.9 & 8.1 & 6.7 & 8.8 \\
\hline Estonia & 13.0 & 5.2 & 3.1 & 4.0 & 0.7 & 19.7 & 6.0 & 5.7 & 4.1 & 3.9 & 17.3 & 5.9 & 4.9 & 4.2 & 2.4 \\
\hline Finland & 24.9 & 8.3 & 6.0 & 6.1 & 4.6 & 29.1 & 8.8 & 7.2 & 6.2 & 6.9 & 28.0 & 9.2 & 6.9 & 6.3 & 5.5 \\
\hline France & 28.4 & 12.5 & 4.6 & 7.5 & 3.8 & 31.0 & 13.0 & 5.1 & 7.6 & 5.4 & 29.9 & 13.0 & 4.9 & 7.7 & 4.4 \\
\hline Germany & 25.2 & 10.7 & 4.0 & 7.8 & 2.7 & 27.3 & 10.5 & 4.1 & 8.1 & 4.6 & 25.8 & 10.4 & 3.9 & 8.2 & 3.2 \\
\hline Greece & 21.3 & 11.9 & 2.0 & 5.9 & 1.6 & 23.2 & 11.8 & 2.3 & 6.0 & 3.1 & 23.1 & 12.0 & 2.4 & 6.1 & 2.5 \\
\hline Hungary & 22.9 & 9.1 & 5.3 & 5.2 & 3.2 & 23.5 & 9.5 & 5.4 & 5.2 & 3.3 & 22.1 & 9.3 & 5.1 & 5.3 & 2.3 \\
\hline Iceland & 14.6 & 1.9 & 3.7 & 5.7 & 3.2 & 15.8 & 2.6 & 5.0 & 5.2 & 3.0 & 14.0 & 2.3 & 4.4 & 4.7 & 2.6 \\
\hline Ireland & 16.3 & 3.6 & 5.3 & 5.8 & 1.6 & 22.8 & 3.7 & 8.1 & 5.9 & 5.2 & 19.8 & 3.7 & 6.9 & 5.9 & 3.3 \\
\hline Israel & 15.5 & 4.8 & 4.0 & 4.3 & 2.3 & 16.3 & 5.1 & 4.3 & 4.2 & 2.5 & 15.7 & 5.0 & 4.2 & 4.0 & 2.5 \\
\hline Italy & 24.9 & 14.1 & 2.8 & 6.6 & 1.4 & 27.5 & 14.1 & 3.1 & 6.6 & 3.7 & 26.4 & 14.1 & 3.0 & 6.7 & 2.6 \\
\hline Korea & 7.6 & 1.7 & 0.8 & 3.5 & 1.5 & 9.0 & 2.4 & 1.4 & 3.8 & 1.3 & 9.7 & 2.6 & 1.4 & 4.3 & 1.4 \\
\hline Luxembourg & 20.6 & 6.5 & 5.7 & 6.4 & 2.1 & 23.5 & 6.5 & 6.6 & 6.5 & 4.0 & 23.6 & 6.6 & 6.5 & 6.6 & 3.9 \\
\hline Mexico & 7.2 & 1.4 & 0.9 & 2.6 & 2.3 & 8.2 & 1.7 & 1.2 & 2.4 & 2.9 & .. & .. & .. & .. &.. \\
\hline Netherlands & 20.1 & 4.7 & 5.3 & 6.0 & 4.1 & 22.6 & 4.6 & 6.1 & 6.1 & 5.8 & 21.5 & 4.8 & 5.9 & 6.2 & 4.6 \\
\hline New Zealand & 18.4 & 4.3 & 5.1 & 7.1 & 1.9 & 21.8 & 5.0 & 5.8 & 8.8 & 2.1 & 21.8 & 5.1 & 5.3 & 9.4 & 1.9 \\
\hline Norway & 20.8 & 4.7 & 5.4 & 5.7 & 5.0 & 24.0 & 5.1 & 6.1 & 5.8 & 7.1 & 22.4 & 5.3 & 5.7 & 5.8 & 5.5 \\
\hline Poland & 19.8 & 10.6 & 3.5 & 4.6 & 1.1 & 21.8 & 9.9 & 3.7 & 4.7 & 3.5 & 21.1 & 9.5 & 3.6 & 4.8 & 3.2 \\
\hline Portugal & 22.5 & 10.8 & 4.0 & 6.6 & 1.1 & 26.1 & 11.2 & 4.7 & 6.7 & 3.4 & 25.4 & 11.3 & 4.8 & 6.8 & 2.5 \\
\hline Slovak Republic & 15.7 & 5.8 & 3.5 & 5.2 & 1.1 & 18.2 & 5.7 & 4.2 & 5.4 & 3.0 & 17.0 & 5.6 & 4.0 & 5.5 & 1.9 \\
\hline Slovenia & 20.3 & 9.6 & 3.9 & 5.6 & 1.1 & 23.9 & 9.8 & 4.6 & 5.8 & 3.7 & 23.7 & 10.0 & 4.6 & 5.9 & 3.2 \\
\hline Spain & 21.6 & 8.0 & 5.1 & 6.1 & 2.4 & 26.7 & 8.5 & 6.6 & 6.2 & 5.3 & 25.3 & 8.6 & 6.4 & 6.2 & 4.1 \\
\hline Sweden & 27.3 & 7.2 & 5.6 & 6.6 & 8.0 & 28.2 & 7.3 & 5.7 & 6.7 & 8.6 & 26.5 & 7.2 & 5.3 & 6.7 & 7.3 \\
\hline Switzerland & 18.5 & 6.4 & 4.3 & 5.6 & 2.2 & 19.6 & 6.3 & 4.8 & 5.6 & 3.0 & 18.5 & 6.4 & 4.5 & 5.2 & 2.3 \\
\hline United Kingdom & 20.5 & 5.4 & 4.5 & 6.8 & 3.8 & 24.4 & 5.4 & 5.5 & 6.9 & 6.5 & 22.9 & 5.5 & 5.3 & 7.0 & 5.1 \\
\hline United States & 16.2 & 6.0 & 2.0 & 7.2 & 1.0 & 20.4 & 6.9 & 3.1 & 8.9 & 1.5 & 19.5 & 6.7 & 2.6 & 8.7 & 1.5 \\
\hline OECD & 19.3 & 6.9 & 4.0 & 5.8 & 2.5 & 22.2 & 7.1 & 4.8 & 6.1 & 4.1 & 22.1 & 7.4 & 4.9 & 6.3 & 3.5 \\
\hline
\end{tabular}

Note: see Annex I.1 for information on data and methodology underlying the estimates.

Source: OECD Social Expenditure database (SOCX, www.oecd.org/els/social/expenditure).

29. Other social services (not including health) capture services to the elderly and disabled, family services, housing, other social policy areas and include ALMPs. The economic crisis resulted in 
higher spending on ALMPs in many countries, including in Denmark, Estonia, Finland, Ireland, Slovenia, Spain and the United Kingdom.

\section{I.5. Private social expenditure: trends and composition}

30. Countries differ strongly in the degree to which their social protection systems rely on private provision. In 2007, gross private social spending was highest at over 10\% of GDP in the United States. By contrast, private social spending as recorded in SOCX amounted to less than 1\% of GDP in the Czech Republic, Estonia, Hungary, Israel, Luxembourg, Mexico, Poland, New Zealand, Spain and Turkey. In some OECD countries, the role of private social benefits has increased in recent years, especially in Canada, Chile, Iceland, Korea and the United States (Chart I.7).

\section{Chart I.7: Gross private social spending has increased since 1990}

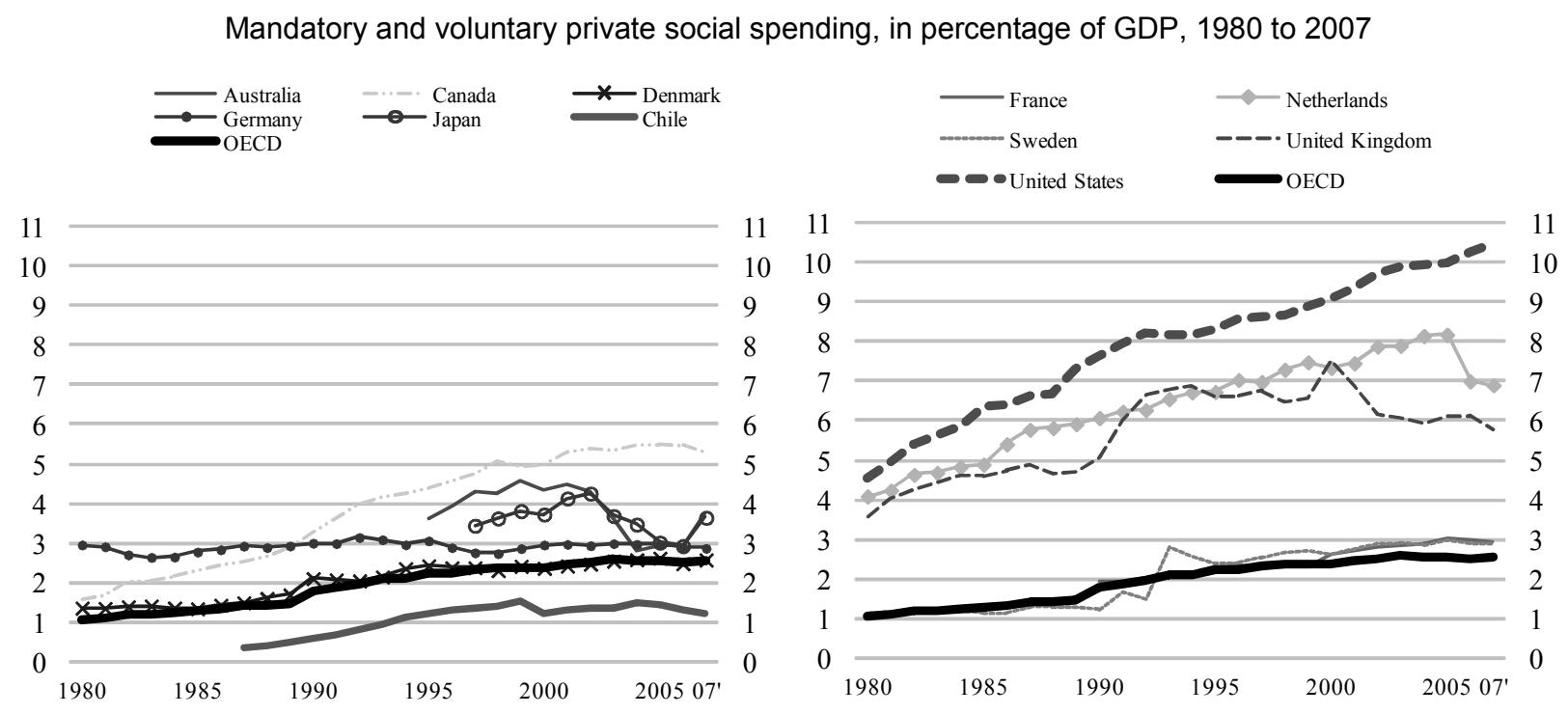

Note: Significant change for Japan and the United Kingdom results from a break in series.

Source: OECD Social Expenditure database (SOCX, www.oecd.org/els/social/expenditure).

31. Private social health spending is a major spending item in the United States and increasing health care costs since the 1980s have contributed to the trend increase in private social spending. In many other countries there is a general upward trend in private social spending driven in large part by the maturing, i.e. an increasing number of pay-outs, of private pension schemes.

32. However, Chart I.7 also shows that in some countries the private social spending-to-GDP ratios have declined over some years. The decline in Australia over the 2001 to 2006 period is largely related to a decline in overall payouts of superannuation lump-sum transfers. There was a downward trend in employer-paid severance payments in Japan, while greater coverage of pension benefit plans underlies the increase in recorded spending for 2007. Health reform in the Netherlands in 2005/06 meant that almost all Dutch residents are now covered by public health arrangements, whereas prior to reform about one-third of residents only had private health coverage. The recorded decline in private pension spending in the United Kingdom around the turn of the millennium does not reflect reform but a break in the series due to a recategorisation of benefits when the new ESSPROS methodology came into force (Eurostat, 2008). With the increasing interest in private social benefits, the recording of relevant programmes is likely to improve. 
33. Spending-to-GDP ratios facilitate a cross-country comparison of current outlays accruing from private pension programmes. However, it provides only a partial view of the importance of private pension saving and does not say anything about current revenue of fully- or partly-funded pension systems, the size of assets held by pension funds or the rate of return on such assets. At present, the working-age population pays more into funded pension systems than is paid out to those in retirement. However, the group of retirees is growing and it is this maturing of pension programmes that partially underlies the upward trend in private social expenditure. Again, SOCX only records pension benefits paid to recipients and not contributions to the system, and hence does not allow for a comparison of total current revenue of public and private pension systems (earnings-related pension contributions and budgetary transfers) with total spending on pension benefits.

34. On average, around $75 \%$ of all private social expenditure takes the form of voluntary spending, with the remainder being mandated by law (Table I.3). Private social benefits are common in the case of occupational accidents and diseases (e.g., Australia), sickness benefits (e.g., Germany) and old-age pensions, in the form of either mandatory participation in employer based programmes (e.g., the United Kingdom), or tax-supported individual pension plans (e.g., the United States), In line with recording practice in the national accounts pensions paid to former civil servants through autonomous funds (e.g., Australia (partially), Canada, Denmark, the Netherlands, Sweden and the United Kingdom) are also recorded as private social spending.

35. Until recently there was no public health insurance system with extensive coverage for workers in the United States. Hence, private health spending is important in the United States: employer-provided health benefits to their workers, dependents and retirees were estimated to be USD 760 billion in 2007 or $5.5 \%$ of GDP (these expenditures do not include payments by individuals for health services). In 2007, total health expenditure was highest in the United States at $15.7 \%$ of GDP, France (11\%) and Belgium (10.8\%), compared to $8.6 \%$ of GDP on average across the OECD (OECD, 2010a). Relatively high health expenditure in the United States leads to total social spending in the United States being close to the OECD average (Table I.3).

36. Non-health private social cash transfers to the working-age population include mandatory employer-provided incapacity-related cash transfers - sickness, disability and occupational injury benefits - as recorded for Australia, Austria, Denmark, Finland, France, Germany, Iceland, Korea, Luxembourg, the Netherlands, Norway, Portugal, Sweden, Switzerland, the United Kingdom and the United States (in some states). Other examples of private social benefits include: supplementary unemployment compensation in the United States, employer-provided childcare support in the Netherlands and employer payments during parental leave periods in many countries, but information on such employer-provided leave payments (and or top-ups) is not available on a cross-national basis. 
Table I.3: Composition of private social spending

Mandatory and voluntary private social expenditure by broad spending category, 2007

\begin{tabular}{|c|c|c|c|c|c|c|c|c|c|c|c|c|}
\hline & \multicolumn{5}{|c|}{ Mandatory private } & \multicolumn{5}{|c|}{ Voluntary private } & \multirow{2}{*}{$\begin{array}{c}\text { Total } \\
\text { private }\end{array}$} & \multirow{2}{*}{$\begin{array}{l}\text { Share of private in total } \\
\text { (public+private) social } \\
\text { spending } \\
\%\end{array}$} \\
\hline & Total & Old age & Incapacity & Health & Other & Total & Old age & Incapacity & Health & Other & & \\
\hline Australia & 0.5 & 0.5 & - & - & - & 3.3 & 2.6 & - & 0.7 & 0.0 & 3.8 & 19.1 \\
\hline Austria & 0.8 & - & 0.8 & - & - & 1.0 & 0.5 & - & 0.5 & - & 1.8 & 6.5 \\
\hline Belgium & 0.0 & 0.0 & 0.0 & - & 0.0 & 4.7 & 2.8 & 0.6 & 0.5 & 0.9 & 4.7 & 15.3 \\
\hline Canada & - & - & - & - & - & 5.3 & 4.1 & - & 1.3 & 0.0 & 5.3 & 23.9 \\
\hline Chile & 1.2 & 0.9 & 0.1 & 0.0 & 0.2 & $\mathrm{a}$ & $\mathrm{a}$ & a & $\mathrm{a}$ & $\mathrm{a}$ & 1.2 & 10.2 \\
\hline Czech Republic & 0.2 & 0.2 & 0.0 & - & 0.0 & 0.2 & 0.1 & 0.1 & 0.0 & 0.0 & 0.4 & 2.0 \\
\hline Denmark & 0.2 & - & 0.2 & - & - & 2.3 & 2.2 & - & 0.2 & 0.0 & 2.6 & 9.0 \\
\hline Estonia & a & $\mathrm{a}$ & $\mathrm{a}$ & $\mathrm{a}$ & $\mathrm{a}$ & 0.0 & $\mathrm{a}$ & $\mathrm{a}$ & 0.0 & 0.0 & 0.0 & 0.1 \\
\hline Finland & - & - & - & - & - & 1.1 & 0.2 & 0.6 & 0.2 & 0.1 & 1.1 & 4.1 \\
\hline France & 0.3 & 0.1 & 0.1 & - & 0.1 & 2.6 & 0.1 & 0.5 & 1.4 & 0.6 & 2.9 & 9.3 \\
\hline Germany & 1.1 & - & 1.0 & - & 0.0 & 1.8 & 0.8 & - & 1.0 & 0.0 & 2.9 & 10.2 \\
\hline Greece & - & - & - & - & - & 1.5 & 0.4 & 0.5 & - & 0.6 & 1.5 & 6.7 \\
\hline Hungary & - & - & - & - & - & 0.2 & - & 0.0 & 0.2 & 0.0 & 0.2 & 0.8 \\
\hline Iceland & 1.6 & - & 1.5 & - & 0.0 & 3.6 & 2.5 & 0.6 & 0.0 & 0.5 & 5.1 & 26.1 \\
\hline Israel & a & $\mathrm{a}$ & a & $\mathrm{a}$ & $\mathrm{a}$ & 0.5 & $\mathrm{a}$ & $\mathrm{a}$ & 0.5 & 0.5 & 0.5 & 2.9 \\
\hline Ireland & - & - & - & - & - & 1.5 & 0.9 & - & 0.6 & 0.0 & 1.5 & 8.5 \\
\hline Italy & 1.6 & 1.1 & 0.3 & - & 0.1 & 0.6 & 0.2 & 0.0 & 0.1 & 0.3 & 2.1 & 7.9 \\
\hline Japan & 0.6 & 0.4 & 0.2 & - & 0.0 & 3.1 & 2.9 & - & 0.2 & 0.0 & 3.6 & 16.3 \\
\hline Korea & 0.6 & 0.4 & 0.1 & - & 0.1 & 2.0 & 0.0 & 0.0 & 0.3 & 1.8 & 2.6 & 25.8 \\
\hline Luxembourg & 0.3 & - & 0.3 & - & - & 0.7 & 0.3 & 0.1 & 0.1 & 0.2 & 0.9 & 4.3 \\
\hline Mexico & - & - & - & - & - & 0.2 & - & - & 0.2 & - & 0.2 & 2.9 \\
\hline Netherlands & 0.6 & 0.0 & 0.6 & - & 0.0 & 6.3 & 4.1 & 0.5 & 0.6 & 1.2 & 6.9 & 25.6 \\
\hline New Zealand & - & - & - & - & - & 0.4 & - & - & 0.4 & - & 0.4 & 2.3 \\
\hline Norway & 1.2 & - & 1.2 & - & - & 0.8 & 0.6 & 0.2 & - & 0.0 & 2.0 & 8.8 \\
\hline Poland & - & - & - & - & - & 0.0 & - & - & 0.0 & - & 0.0 & 0.2 \\
\hline Portugal & 0.4 & - & 0.4 & - & - & 1.5 & 0.5 & 0.1 & 0.4 & 0.5 & 1.9 & 7.7 \\
\hline Slovak Republic & 0.1 & 0.1 & 0.0 & - & 0.0 & 0.8 & 0.5 & 0.1 & - & 0.3 & 1.0 & 6.0 \\
\hline Slovenia & $\mathrm{a}$ & $\mathrm{a}$ & a & $\mathrm{a}$ & $\mathrm{a}$ & 1.0 & 0.0 & 0.0 & 1.0 & 0.0 & 1.0 & 4.8 \\
\hline Spain & - & - & - & - & - & 0.5 & - & - & 0.5 & - & 0.5 & 2.2 \\
\hline Sweden & 0.4 & - & 0.4 & - & - & 2.5 & 2.1 & 0.3 & 0.0 & 0.1 & 2.9 & 9.5 \\
\hline Switzerland & 7.2 & 5.3 & 1.1 & - & 0.8 & 1.1 & 0.0 & 0.0 & 1.0 & 0.0 & 8.3 & 30.8 \\
\hline Turkey & - & - & - & - & - & - & - & - & - & - & - & - \\
\hline United Kingdom & 0.8 & 0.6 & 0.0 & - & 0.2 & 5.0 & 3.9 & 0.4 & 0.1 & 0.6 & 5.8 & 22.0 \\
\hline United States & 0.3 & - & 0.2 & 0.1 & - & 10.2 & 4.3 & 0.3 & 5.5 & 0.0 & 10.5 & 39.3 \\
\hline OECD & 0.6 & 0.3 & 0.3 & 0.0 & 0.0 & 1.9 & 1.1 & 0.1 & 0.5 & 0.2 & 2.5 & 10.9 \\
\hline
\end{tabular}

Note: - Zero.

Source: OECD Social Expenditure database, www.oecd.org/els/social/expenditure.

\section{I.6 Net (after tax) social expenditure}

37. The detailed social expenditure programme data discussed above is indispensable for in-depth monitoring of welfare policy trends and changes therein, but they do not take account of tax systems' effect on public and private spending on social protection. And as the overall effect can be considerable and vary across countries, it significantly affects cross-national comparisons of social expenditure. 
38. Broadly speaking, tax systems affect levels of social expenditure in three ways:

1. Direct taxation of benefit income: Governments levy income tax and social security contributions on cash transfers to beneficiaries, in which case redistribution of resources is lower than suggested by gross spending indicators.

2. Indirect taxation of consumption by benefit recipients: Benefit income is provided to finance consumption of goods and services. Indirect taxes reduce the consumption which can be financed out of a given level of benefit income.

3. Tax breaks for social purposes: Governments also make use of the tax system to directly pursue social policy goals. Fiscal measures with social effects are those which can be seen as replacing cash benefits (e.g., child tax allowances) or stimulating the provision of private benefits (e.g., tax relief towards the provision of private health plans). These tax breaks for social purposes (TBSPs) can be directly awarded to households, but also include tax relief for employers and private funds that ultimately benefit households (e.g., favourable tax treatment of employer-benefits provided to households, favourable tax treatment of private funds).

39. The adjustments for direct and indirect taxation of benefits do not affect service spending, even though such services,(e.g., pharmaceutical products), can be subject to indirect taxation. Data on spending on social services that are subject to indirect taxation and at what rate is not available on a comprehensive basis.

\section{I.6.1. The value of direct taxation of transfer income in aggregate terms}

40. In some OECD countries benefits are taxed in the same way as earnings while in other countries most benefits are taxed at a reduced rate. In yet other countries, almost all benefits are paid net of direct taxation. For example, gross payments to an unemployed worker with a partner and 2 children in Sweden would be higher than in Austria, but net income for such a family in Sweden is slightly lower than in Austria. In aggregated spending terms, net (after tax) public spending on unemployment benefits is about $70 \%$ of the level suggested by gross indicators in Sweden.

41. Taxation can also varies by benefit type: unemployment assistance, social assistance, housing benefits and family benefits are frequently not taxed in countries. By contrast, public and private retirement and disability pension payments are generally taxed, but frequently at reduced rates, while continued wage payments in case of absence due to sickness are taxed as earnings (see Part II below).

42. There are large differences in the level of direct taxes and social security contributions paid by recipients of social benefits across countries. Chart I.8 Panel A shows that in 2007, direct tax and social security contributions paid by benefit-recipients amounted to around $27 \%$ of gross public spending on cash transfers in Denmark and Sweden. On average, just over 9\% of public transfer income is clawed back through the tax system in OECD countries. Private benefit income is generally taxed at a higher rate than public transfer income (on average around 11\%): private benefit income is taxed at more than $20 \%$ in Finland, Germany, the Netherlands, Norway and Sweden, and at almost 35\% in Denmark.

43. Direct taxation of benefit income in the Czech Republic, Korea, Mexico, the Slovak Republic and Turkey is negligible, and the value of direct taxation of public benefit income is also below $1 \%$ of GDP at factor cost in Australia, Canada, Iceland, Ireland, Japan, the United Kingdom and the United States.

44. Chart I.8 Panel B shows that direct tax paid by benefit recipients in Denmark and Sweden amounted to about $3.4 \%$ of GDP in 2007. Direct tax paid by public benefit recipients exceeds $2.0 \%$ of 
GDP in Austria, Italy and Finland. It is around OECD average at 1.1\% GDP in Belgium, France, New Zealand, Spain, Germany and Luxembourg, while this is less than $0.5 \%$ of GDP in Australia, Canada (without fully accounting for direct taxes across Provinces), Ireland, Japan and the United Kingdom. Tax paid by public benefit recipients is negligible in the Czech Republic and Korea, and benefits are tax-free in Mexico and in the Slovak Republic. As private transfer spending is considerably smaller than public transfer spending, the amount of tax paid over private benefit income is relatively small, being at its highest in the Netherlands at just over $1.3 \%$ of GDP.

\section{I.6.2. The importance of indirect taxation of consumption out of benefit income}

45. Social benefits are given in order to finance consumption of goods and services such as housing, food, clothing and so on. Governments tax the consumption of different goods and the amounts involved are substantial. For example, in Finland value-added tax receipts were worth EUR 15.0 billion in 2007; in the same year in France, duties on the consumption of electricity and heating (gas) amounted to about EUR 2.6 billion, while those on water consumption were EUR 1.7 billion (OECD, 2010b).

46. Consumption taxes reduce the real value of consumption which can be financed out of a given level of benefits, and (as with direct taxation of benefit income) establish another flow back in tax receipts to the government. Similarly to differences in direct taxation of benefit income, cross-country differences in indirect taxation affect comparisons of welfare state spending. In countries where indirect taxation is relatively limited (i.e., in non-European OECD countries), gross spending levels can also be relatively low to generate the same net income level for benefit recipients in countries with high indirect tax rates. In some countries, policy explicitly recognises the impact of indirect taxation on the financial position of lowincome households (many of whom receive transfer income). For example, when the Goods and Services Tax was introduced in Australia in July 2000 at a rate of 10\% (with food being exempt), a compensation package for social protection benefit recipients was introduced at the same time. Similarly, Canada has a Goods and Services Tax rebate to support low-income households.

47. To some extent the relatively low social spending-to-GDP ratios in the United States and in other non-European OECD countries are related to the low indirect tax levels that prevail in these countries. Accounting for this feature improves the quality of cross-country comparisons of social spending, and estimates on its importance are derived from the OECD National Accounts (OECD, 2010c) and the OECD Revenue Statistics (OECD, 2010b, and Table II.4 in Part II, below).

48. On the basis of the indirect tax base used for this study (see Section II.4.2 in Part II below), in 2007, indirect taxes were lowest in the United States (3.9\%), Mexico (6.0\%) and Japan (6.1\%) and were around 9\% in Australia and Canada (Chart I.9 Panel A). Indirect taxes ranged from 11-14\% in Germany, Korea, Italy and Spain and ranged from $15-22 \%$ in most other European countries. Indirect taxation levied on consumption of benefit income was about $1.6 \%$ of GDP on average across the OECD, and was highest in Austria, Denmark and Luxembourg at over $2.5 \%$ of GDP. It was lowest in Korea, Mexico and the United States (Chart I.9 Panel B). This implies that net transfers from government to households, particularly in European countries are rather less than gross expenditure figures suggest. Since low indirect tax rates generally prevail in low social spending countries, this also leads to a reduction of variation in net spending levels across countries. 


\section{Chart I.8: A large tax burden on benefit income in Denmark and Sweden}

A. Direct taxes paid by recipients of public/private benefits, in percentage of gross public/private social spending in cash, in 2007

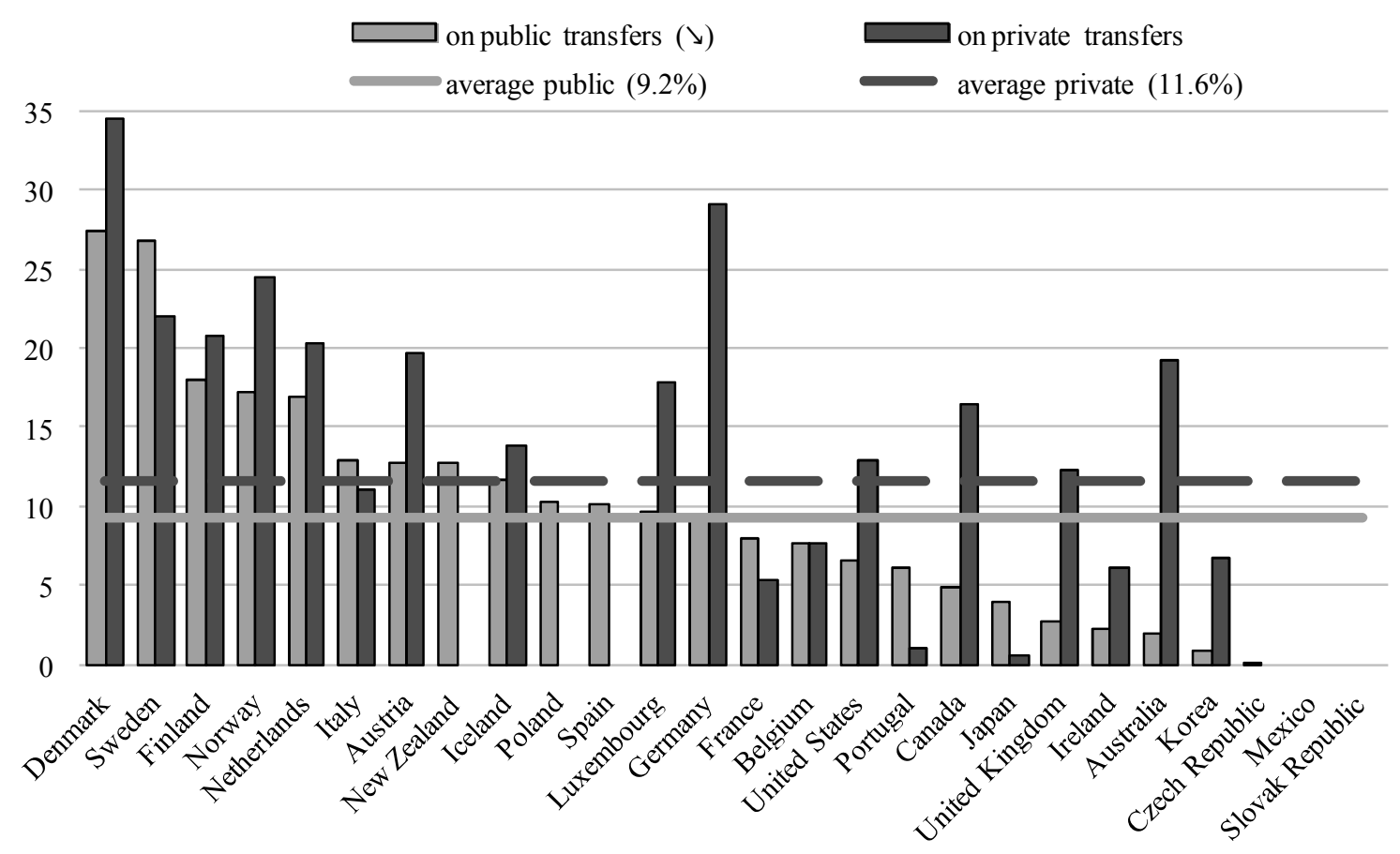

B. Direct taxes paid by recipients of public/private benefits, in percentage of GDP, in 2007

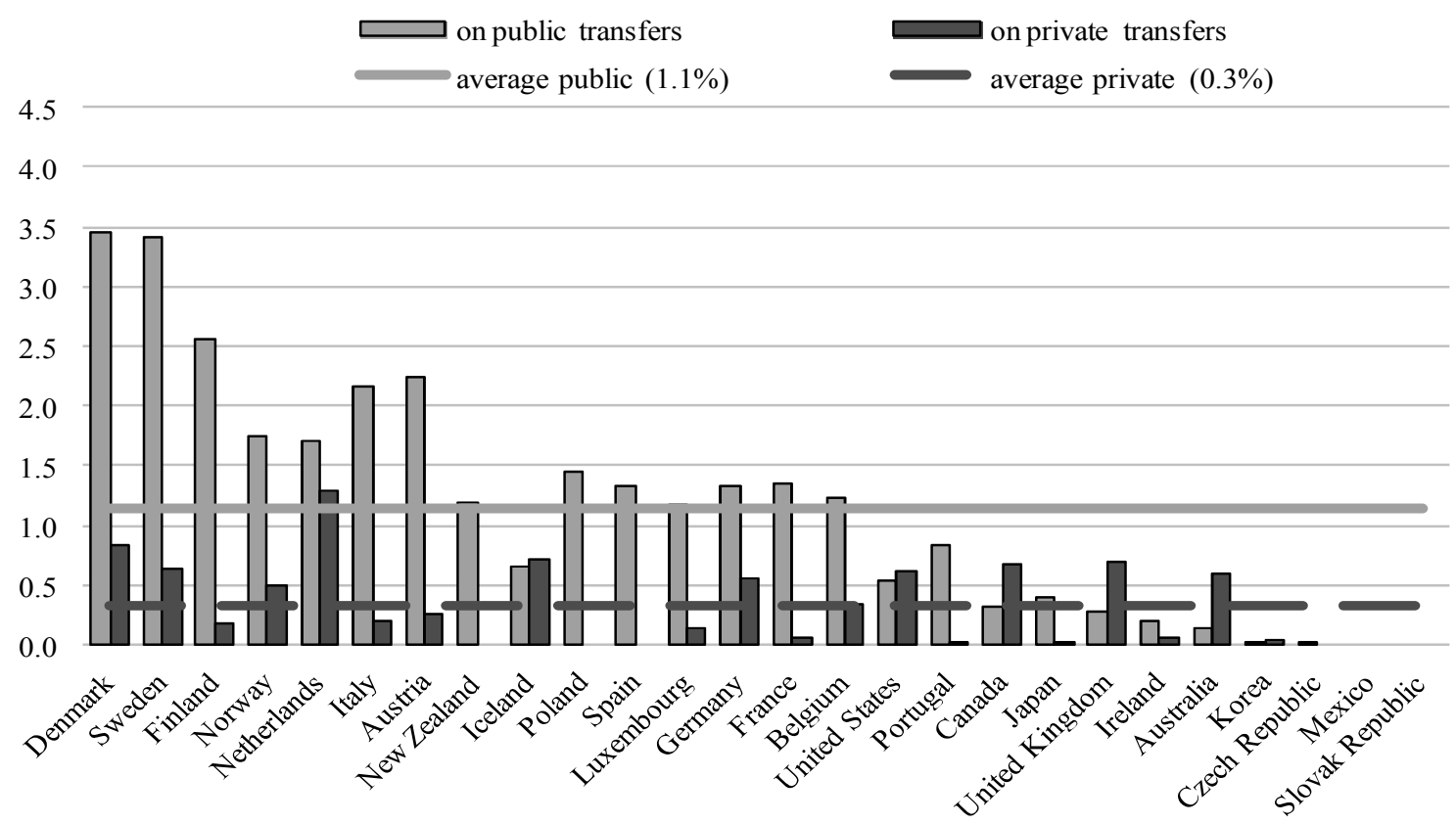

Source: See Annex I.2. 


\section{Chart 1.9: Indirect taxation is least important in Non-European OECD countries}

A. Indirect taxes paid by recipients of public/private benefits in per cent of gross public/private social spending in cash, 2007

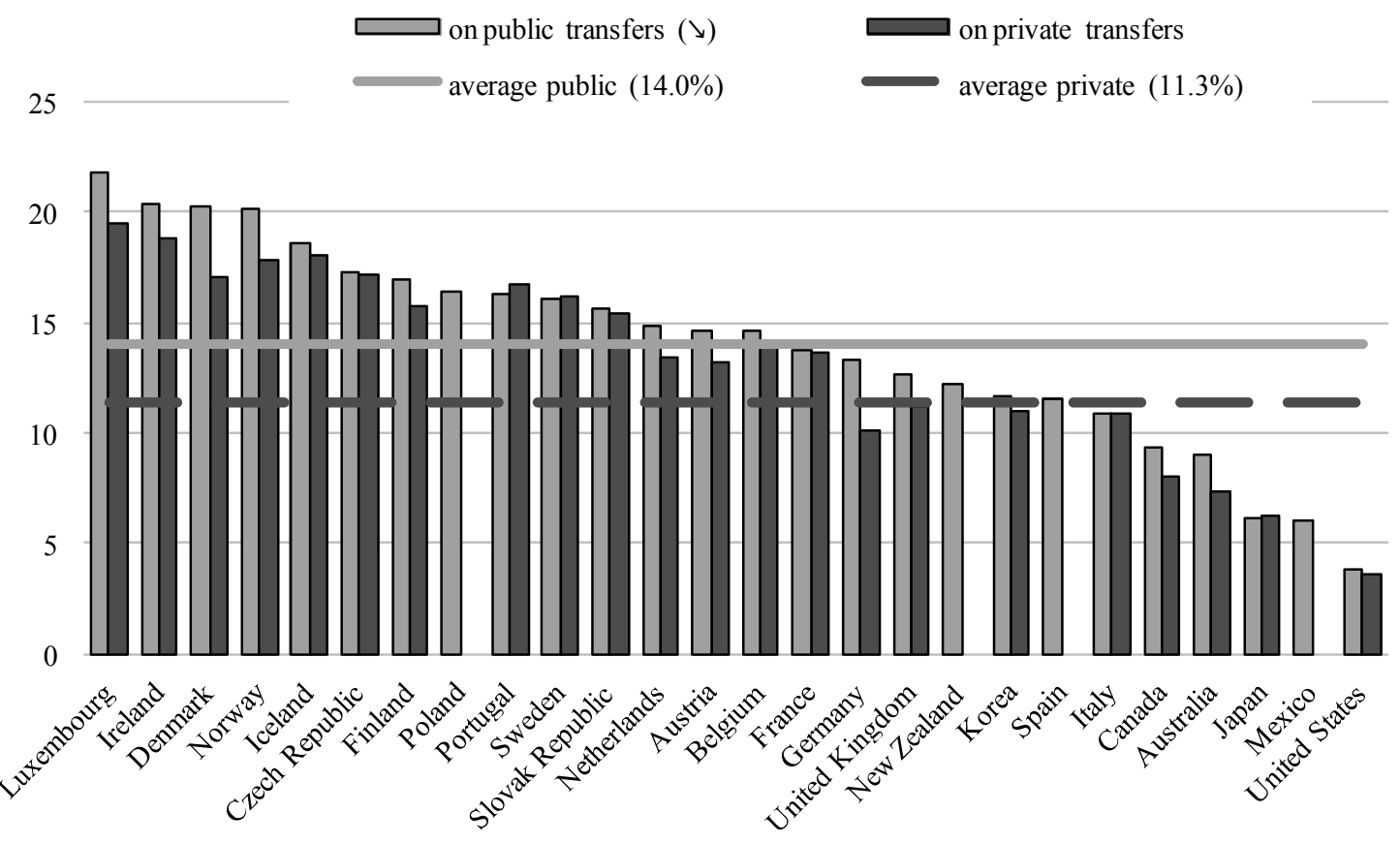

B. Indirect taxes paid by recipients of public/private benefits, in percentage of GDP, in 2007

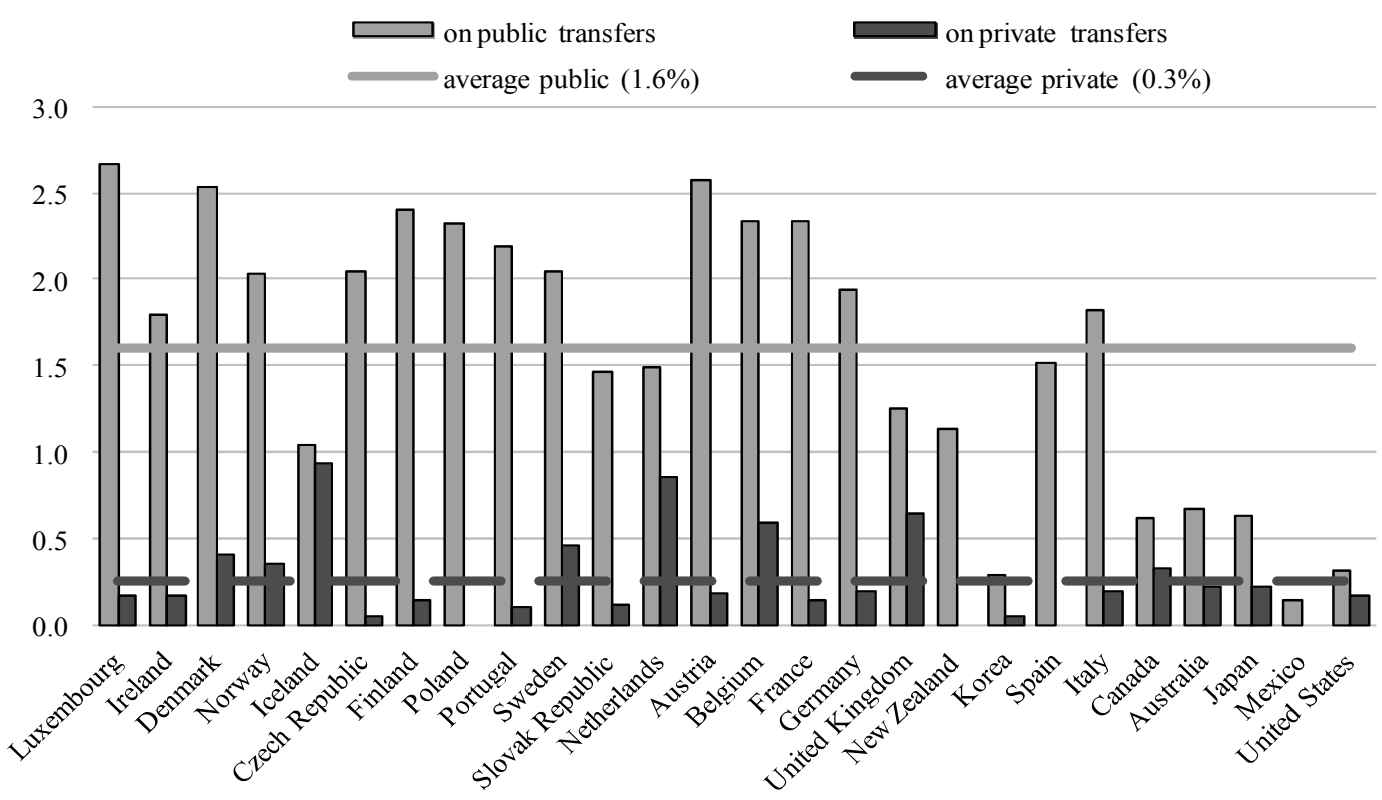

Source: See Annex I.2. 


\section{I.6.3. Tax breaks for social purposes}

49. Many governments of OECD countries pursue social policy objectives through the tax system, sometimes by reducing taxation on particular sources of income, which is already reflected in the variation of direct taxation of benefit income as discussed above. By contrast, Tax Breaks for Social Purposes (TBSPs) are defined as:

"those reductions, exemptions, deductions or postponements of taxes, which: a) perform the same policy function as transfer payments which, if they existed, would be classified as social expenditures; or $b$ ) are aimed at stimulating private provision of benefits".

50. Tax breaks that are similar to cash benefits can be substantial and often concern support for families. ${ }^{3}$ For example, value of support to children in France through the 'quotient familial' was around EUR 11.5 billion in 2007 (Annex I.2). Sometimes, fiscal support and cash transfers (i.e., non-wasteable tax credits $^{4}$ ) for families are an integral part of the same social programme, with cash payments recorded in the OECD Social Expenditure database 5 and fiscal support in the OECD Revenue Statistics. For example, in Germany in 2007 tax relief for children amounted to EUR 36.6 billion (Annex I.2), of which EUR 20.9 billion was off-set against tax liabilities (and thus recorded as a TBSP) and EUR 15.7 billion paid out in transfer income, and thus recorded as a cash transfer. Similarly, for the United Kingdom GBP 4.7 billion spent under the WTC/CTC programme was recorded as a TBSP in 2007, while GBP 15.4 billion is recorded as gross transfer spending. In 2007, the cost of the Earned Income Tax Credit in the United States amounted to USD 43.3 billion, of which USD 5.0 billion in the form of tax credits that mirror cash benefit, while USD 38.3 billion concerned tax credits exceeding tax liabilities of recipients.

51. Governments sometimes also use the tax system to stimulate the take-up of private social insurance coverage by individuals and/or employment-related plans. These tax breaks can be categorised in two broad groups. First, there are 'Tax breaks towards current private social benefits', i.e., favourable tax treatment aimed at stimulating the provision of private social benefits in the current year such as voluntary private unemployment coverage or private health insurance. This type of tax break is important in Germany (where about $18 \%$ of the population is covered by private health insurance) and particularly in the United States, where the exclusion of employer contributions for medical insurance premiums and medical care amounted to USD 133.8 billion in 2007, equivalent to $1.0 \%$ of GDP (Chart I.10). Tax breaks towards current private social benefits also include favourable treatment of contributions to and income of NGOs. Again this form of fiscal support is most prevalent in the United States where deductibility of contributions to charities amounted to USD 38.2 billion in 2007, or 0.3\% of GDP (Annex I.2).

52. The second group of tax breaks towards private benefits is arguably the most important. However, there is no comparable data set available on the value of tax breaks for pensions, as underlying calculations are complex and methods are not standardized across countries. Therefore, the data that is

3 Governments thus make ample use of tax systems to support families with children, and accounting for relevant fiscal support thus allows to consider public support on family benefits in a comprehensive manner, i.e., accounting for cash transfers, spending on services (e.g., childcare) and fiscal support (OECD Family database- Indicator PF1.1, www.oecd.org/els/social/family/database).

In case of a 'wasteable' (or 'non-refundable') tax credit, entitlements only accrue to the extent that they are off-set against tax liabilities, while 'non-wasteable' or 'refundable' tax credits involve cash transfers to people (e.g., low-income workers) whose tax liabilities are not large enough to make (full) use of a particular entitlement (tax credit). Non-wasteable tax credits thus reinforce the re-distributive nature of a tax/benefit system.

5. Despite its name the Canada Child Tax Benefit is delivered and recorded as a cash payment in SOCX as child payments by the fiscal authorities in Austria are recorded as a cash transfer, not as fiscal support. 
available on the cost to public budgets of tax reliefs to private pension plans is only presented as a memorandum item. Available information for 2001, 2003, 2005 and 2007 (Table I.4 and Annex I.2) shows that the value of favourable tax treatment of private pension arrangements was in excess of $1 \%$ of GDP in Australia, Canada, Ireland, the Netherlands and the United States (estimates for previous years suggest this type of support is also important in the United Kingdom). These are also the countries where private pension benefits are most important. ${ }^{6}$

\section{Chart I.10: A high value of TBSPs in the United States, while they are virtually non-existent in Scandinavian countries}

Tax breaks with a social purpose in percentage of GDP, 2007

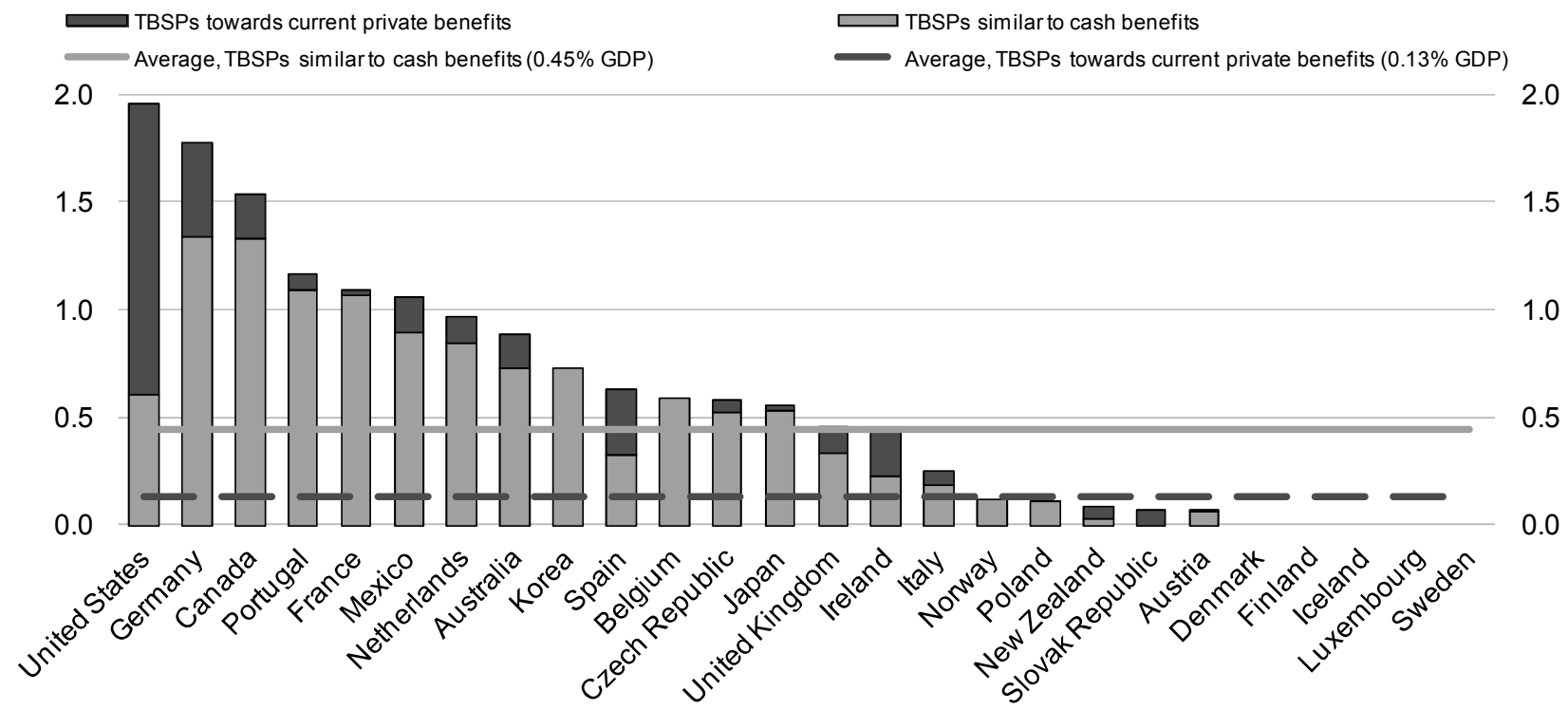

Source: See Annex I.2.

\section{I.6.4. The overall effect of tax systems on social spending}

53. Table I.4 pulls together information on the importance of different social expenditure and tax items in each country (the OECD SOCX Manual in Part II describes the framework in detail). Gross public social expenditure indicators (Table I.4, line 1) lead us to believe that public social expenditure in Nordic countries (30\% of GDP at factor costs) and Europe in general (26\%) is much higher than in non-European OECD countries $(17 \%)$.

54. In general, governments claw back more money through direct and indirect taxation of public transfer income than the value of the tax advantages awarded for social purposes:

6. It is difficult to be precise on the extent to which tax advantages are instrumental in stimulating private coverage. Tax breaks certainly affect individual behaviour and provide governments with a tool to influence take-up of particular plans, but may not lead to much additional saving on a national basis. For example, in the late 1980s individual retirement accounts were introduced in the United States. Favourable tax treatment certainly increased the coverage of this programme, but as in $1990,82 \%$ of all programme contributions were 'rollover contributions' from other employment-based pension plans, the effect on overall pension savings was limited (Adema and Einerhand, 1998). 
- Direct taxes and social security contributions. There is considerable variation across countries in taxation of social transfers: Direct taxation of public benefit income is negligible and/or below $1 \%$ of GDP in about one third of OECD countries, in sharp contrast to Denmark and Sweden where the claw-back on public social transfers through direct taxation is around $4 \%$ of GDP at factor cost. ${ }^{7}$ The value of direct taxation of mandatory private incapacity-related benefits (often taxed as wages) is most significant in Germany, Iceland, and Norway at $0.3 \%$ of GDP or more. Compared to practice in the other countries, the value of direct tax levied over private social benefits is highest in the Netherlands at $1.3 \%$ of GDP.

- Indirect taxes. The value of benefit income clawed back through taxes on consumption is much larger in European countries and in Denmark in particular, than in Australia, Canada, and in particular, Japan, Korea, Mexico and the United States, where indirect tax rates on consumption out of benefit income is significantly lower.

- Tax breaks for social purposes (excluding pensions). These are generally least important in countries with relatively high direct tax levies, including Denmark, Finland, Iceland, Luxembourg, and Sweden, and their value is also limited in Austria (where support for families through the tax system is paid out in cash), New Zealand, Norway, Poland, the Slovak Republic and Turkey. Tax breaks similar to cash benefits are worth over $1.0 \%$ of GDP in Canada, France, Germany and Portugal. Tax breaks towards current private spending arrangements (health insurance) are largest in the United States at around 1.4\% of GDP at factor cost.

55. Thus, net public social expenditure is usually less than gross spending indicators suggest: average gross public spending amounts to $22.4 \%$ of GDP at factor cost for the countries for which data is available, and net public social spending averages 19.9\%. In Austria, Finland, Italy, Luxembourg, Norway and Poland, net spending is around $4 \%$ or more below gross spending levels, the adjustments for taxation imply that net public social spending as a proportion of GDP at factor costs in Sweden and Denmark is 6 to 7 percentage points of GDP below gross spending levels. In Australia and Japan, gross and public net spending levels are virtually the same while in Mexico and the United States gross public spending actually underestimates public social effort by more than 1 percentage point of GDP (Table I.4, lines 1 and $6)$.

56. Table I.4 also reveals that low gross public spending countries (around $20 \%$ of GDP or less) impose limited direct taxation on benefit income (Australia, Canada, the Czech Republic, Iceland, Ireland, Japan, Korea, Mexico, the Slovak Republic, Turkey and the United States), but that the opposite does not always hold true. Countries that claw back less than $2 \%$ of GDP in direct taxation include the United Kingdom (with gross spending around the OECD average) and, particularly, France and Germany (countries with gross spending levels well above the average). Indeed, because France and Germany are high gross public spending countries with a relatively limited tax burden on benefit income compared to most other European countries, they have the highest level of net government social effort.

57. Accounting for the impact of the tax system on social benefits also increases the importance of social services (including health care) vis-à-vis cash transfers. The 'service to cash spending ratio' increases from on average $90 \%$ (gross public social expenditure) to just over 108\% when net public social expenditure is considered. When fiscal measures are accounted for, spending on social services (including health) exceeds spending on transfers in Australia, Canada, Denmark, Finland, Iceland, Korea, Mexico, the Netherlands, New Zealand, Norway, Sweden and the United Kingdom.

7 Net social spending totals are corrected for indirect taxation, and therefore it is most appropriate to relate these indicators to GDP at factor costs which also does not include the value of indirect taxation (see Part II below). 
58. In general, gross and net spending trends move in the same direction with changes in net spending levels generally being the smaller of the two (Annex I.2). However, gross and net spending trends diverge on one occasion, in Denmark from 1993 to 1995: while gross public spending increased from $32.3 \%$ of GDP in 1993 to $33.3 \%$ in 1995 , net spending decreased from $28.4 \%$ of GDPfc to $24.5 \%$ over the same period. The divergence in trends reflects reforms which made old-age pensions and social assistance benefits taxable, whilst raising the gross payment rates of these benefits in order to compensate recipients. As a result, gross spending increased, while in real terms changes were small.

\section{I.6.5. Social spending from the perspective of households}

59. To get a picture of the amount of resources devoted to meeting social needs in a country, both net public and net private social benefits should be considered, although it should be borne in mind that the quality of data on the impact of tax systems and private social spending is not as high as the quality of information on budgetary allocations. Table I.4 line 13 shows that net total social expenditure is highest in France (one third of GDP at factor cost), followed closely by Belgium, Germany and Sweden. Net total expenditure is lowest in Mexico, Turkey and Korea at around 9, 11, and 12\% of GDP at factor costs, and below 21\% in the Slovak Republic, Ireland, Poland, New Zealand, the Czech Republic, Luxembourg, Iceland and Norway. Recipients of social benefits in more than one-third of countries all claim about one quarter of the economy's domestic production (with a margin of variation of 2.5 percentage points of GDP above and below $25 \%$ of GDP).

60. Overall, the results lead to the following general conclusions:

- Accounting for private social benefits and the impact of the tax system on social expenditure has an equalising effect on levels of social expenditure to GDP ratios across the countries considered.

- Except for Australia, Canada, Japan, Korea, Mexico, Turkey and the United States, public social spending is significantly below the levels suggested by gross expenditure data. This is because most countries have significant taxes on social benefits.

- Accounting for both the tax system and the role of private social benefits reveals that social spending levels are similar in countries often thought to have very different gross public social expenditure levels. For example, total net social spending in Austria, Canada, Denmark, Finland, Italy, Japan, the Netherlands, Portugal, the United Kingdom and the United States are within a few percentage points of each other.

61. Moving from gross public to net total social expenditure not only leads to greater similarity in spending levels across countries it also changes the ranking among countries. Denmark, Finland, Norway, Luxembourg, and Spain drop more than five places in the rankings (Chart I.11) and all these countries tax benefits and associated consumption above the OECD average (Table I.4). New Zealand and Spain drop 5 places as they tax benefits around the OECD average but have limited private social spending (Table I.4).

62. By contrast, Chart I.11 shows that Australia, Canada, Germany, Iceland, Japan, the Netherlands, the United Kingdom and the United States move up the rankings by 4 or more places. The reasons differ: Japan and Germany obtain higher ranks because of the limited taxation of benefits: in the other five countries, the role of private social (pension) spending drives up the ranking (Table I.4). As private social spending is so much larger in the United States compared with other countries its inclusion moves the United States to $5^{\text {th }}$ place when comparing net total social spending across countries. 


\section{Table I.4: From gross public to net total social spending, 2007}

Social expenditure indicators as a per cent of GDP at factor cost

\begin{tabular}{|c|c|c|c|c|c|c|c|c|c|c|c|c|c|c|c|c|c|c|c|c|c|c|c|c|c|c|c|c|c|}
\hline & 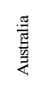 & 营 & 离 & $\begin{array}{l}\text { 总 } \\
\text { 竎 }\end{array}$ & 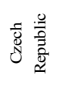 & $\overline{\bar{z}}$ & 崫 & 总 & 衰 & 曾 & 莺 & 童 & 畫 & $\frac{\sqrt{3}}{2}$ & 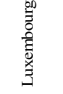 & $\frac{8}{\frac{8}{2}}$ & 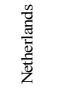 & 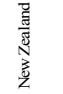 & 竞 & 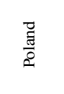 & & 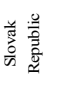 & 䜌 & $\begin{array}{l}\text { 离 } \\
\frac{\mathrm{v}}{\bar{n}}\end{array}$ & 兽 & 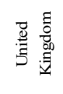 & 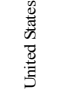 & 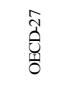 & 8 \\
\hline 1 Gross public social expenditure & 17.9 & 29.6 & 29.6 & 18.9 & 20.7 & 30.8 & 28.2 & 32.8 & 28.4 & 17.5 & 18.6 & 28.8 & 20.3 & 8.6 & 23.2 & 8.0 & 22.7 & 20.9 & 23.3 & 22.8 & 25.6 & 17.4 & 24.1 & 32.1 & 12.1 & 23.3 & 17.4 & 22.4 & $30 \%$ \\
\hline Rank (country ranking, from highest spender to lowest) & 21 & 5 & 4 & 19 & 17 & 3 & 8 & 1 & 7 & 22 & 20 & 6 & 18 & 26 & 13 & 27 & 15 & 16 & 11 & 14 & 9 & 24 & 10 & 2 & 25 & 12 & 23 & & \\
\hline Direct taxes and social contributions & 0.2 & 2.5 & 1.4 & 0.4 & 0.0 & 4.1 & 2.9 & 1.6 & 1.5 & 0.8 & 0.2 & 2.5 & 0.4 & 0.0 & 1.3 & 0.0 & 1.9 & 1.4 & 1.9 & 1.7 & 1.0 & 0.0 & 1.5 & 4.0 & 0.0 & 0.3 & 0.6 & 1.3 & \\
\hline 2 Net cash direct public social expenditure & 17.7 & 27.1 & 28.3 & 18.5 & 20.7 & 26.7 & 25.3 & 31.2 & 26.9 & 16.8 & 18.4 & 26.3 & 19.9 & 8.5 & 21.9 & 8.0 & 20.8 & 19.6 & 21.4 & 21.1 & 24.6 & 17.4 & 22.6 & 28.1 & 12.1 & 23.0 & 16.8 & & \\
\hline Indirect taxes (on cash benefits) & 0.7 & 2.9 & 2.6 & 0.7 & 2.2 & 3.0 & 2.7 & 2.7 & 2.2 & 1.3 & 2.1 & 2.1 & 0.7 & 0.3 & 3.0 & 0.2 & 1.7 & 1.3 & 2.3 & 2.7 & 2.5 & 1.6 & 1.7 & 2.4 & 0.8 & 1.4 & 0.3 & 1.8 & \\
\hline 3 Net direct public social expenditure & 17.0 & 24.2 & 25.6 & 17.8 & 18.4 & 23.7 & 22.6 & 28.5 & 24.7 & 15.5 & 16.3 & 24.2 & 19.2 & 8.2 & 18.9 & 7.8 & 19.1 & 18.3 & 19.1 & 18.5 & 22.1 & 15.7 & 20.9 & 25.7 & 11.3 & 21.6 & 16.5 & & \\
\hline + T1 TBSPs similar to cash benefits & 0.8 & 0.1 & 0.7 & 1.5 & 0.6 & 0.0 & 0.0 & 1.2 & 1.5 & 0.0 & 0.3 & 0.2 & 0.6 & 0.8 & 0.0 & 1.0 & 1.0 & 0.0 & 0.1 & 0.1 & 1.3 & 0.0 & 0.4 & 0.0 & 0.0 & 0.4 & 0.7 & & \\
\hline Indirect taxes & 0.1 & 0.0 & 0.1 & 0.1 & 0.1 & 0.0 & 0.0 & 0.2 & 0.2 & 0.0 & 0.1 & 0.0 & 0.0 & 0.1 & 0.0 & 0.1 & 0.2 & 0.0 & 0.0 & 0.0 & 0.2 & 0.0 & 0.0 & 0.0 & 0.0 & 0.0 & 0.0 & & \\
\hline + T2 TBSPs towards current private benefits & 0.2 & 0.0 & 0.0 & 0.2 & 0.1 & 0.0 & 0.0 & 0.0 & 0.5 & 0.0 & 0.3 & 0.1 & 0.0 & 0.0 & 0.0 & 0.2 & 0.1 & 0.1 & 0.0 & 0.0 & 0.1 & 0.1 & 0.3 & 0.0 & 0.0 & 0.1 & 1.4 & & \\
\hline 5 Net TBSPs (not including pensions) & 0.9 & 0.1 & 0.6 & 1.6 & 0.5 & 0.0 & 0.0 & 1.1 & 1.8 & 0.0 & 0.5 & 0.3 & 0.6 & 0.7 & 0.0 & 1.1 & 0.9 & 0.1 & 0.1 & 0.1 & 1.1 & 0.1 & 0.7 & 0.0 & 0.0 & 0.5 & 2.1 & 0.6 & \\
\hline 6 Net current public social expenditure & 17.9 & 24.2 & 26.2 & 19.4 & 19.0 & 23.7 & 22.6 & 29.6 & 26.5 & 15.5 & 16.8 & 24.4 & 19.7 & 8.9 & 18.9 & 8.9 & 20.0 & 18.4 & 19.2 & 18.6 & 23.2 & 15.8 & 21.6 & 25.7 & 11.3 & 22.0 & 18.6 & 19.9 & $25 \%$ \\
\hline Rank (country ranking, from highest spender to lowest) & 21 & 6 & 3 & 14 & 16 & 7 & 9 & I & 2 & 24 & 22 & 5 & 13 & 26 & 17 & 27 & 12 & 20 & 15 & 19 & 8 & 23 & ${ }_{11}$ & 4 & 25 & 10 & 18 & & \\
\hline 7 Gross mandatory private soc. Exp. & 0.5 & 0.9 & 0.0 & 0.0 & 0.3 & 0.3 & 0.0 & 0.4 & 1.2 & 1.9 & 0.0 & 1.8 & 0.6 & 0.7 & 0.3 & 0.0 & 0.7 & 0.0 & 1.4 & 0.0 & 0.5 & 0.2 & 0.0 & 0.5 & 0.0 & 0.9 & 0.3 & 0.5 & \\
\hline Direct taxes and social contributions & 0.1 & 0.2 & 0.0 & 0.0 & 0.0 & 0.1 & 0.0 & 0.0 & 0.4 & 0.3 & 0.0 & 0.2 & 0.0 & 0.0 & 0.1 & 0.0 & 0.2 & 0.0 & 0.4 & 0.0 & 0.0 & 0.0 & 0.0 & 0.1 & 0.0 & 0.1 & 0.0 & & \\
\hline Indirect taxes & 0.0 & 0.1 & 0.0 & 0.0 & 0.0 & 0.0 & 0.0 & 0.1 & 0.1 & 0.3 & 0.0 & 0.2 & 0.0 & 0.1 & 0.1 & 0.0 & 0.1 & 0.0 & 0.2 & 0.0 & 0.1 & 0.0 & 0.0 & 0.1 & 0.0 & 0.1 & 0.0 & & \\
\hline 8 Net current mand. private soc. exp. & 0.4 & 0.6 & 0.0 & 0.0 & 0.2 & 0.1 & 0.0 & 0.3 & 0.7 & 1.3 & 0.0 & 1.4 & 0.5 & 0.6 & 0.2 & 0.0 & 0.4 & 0.0 & 0.8 & 0.0 & 0.4 & 0.2 & 0.0 & 0.3 & 0.0 & 0.7 & 0.3 & 0.3 & \\
\hline 9 Net publicly mandated soc. exp. $\mid 6+8]^{\mathrm{a}}$ & 18.3 & 24.8 & 26.2 & 19.4 & 19.2 & 23.9 & 22.6 & 29.9 & 27.2 & 16.8 & 16.8 & 25.8 & 20.3 & 9.5 & 19.1 & 8.9 & 20.4 & 18.4 & 20.0 & 18.6 & 23.6 & 16.0 & 21.6 & 25.9 & 11.3 & 22.7 & 18.9 & 20.2 & $25 \%$ \\
\hline Indirect taxes & 0.2 & 0.1 & 0.7 & 0.4 & 0.0 & 0.4 & 0.2 & 0.1 & 0.1 & 0.8 & 0.2 & 0.0 & 0.2 & 0.0 & 0.1 & 0.0 & 0.9 & 0.0 & 0.2 & 0.0 & 0.0 & 0.1 & 0.0 & 0.5 & 0.0 & 0.6 & 0.2 & & \\
\hline 11 Net current voluntary private soc. exp. & 2.9 & 0.9 & 4.3 & 4.8 & 0.2 & 1.4 & 0.8 & 2.8 & 1.7 & 2.9 & 1.5 & 0.6 & 3.2 & 2.3 & 0.5 & 0.2 & 5.0 & 0.5 & 0.5 & 0.0 & 1.5 & 0.8 & 0.6 & 1.8 & 0.0 & 4.4 & 10.1 & 2.1 & \\
\hline 12 Net current private soc. exp. $[8+11]$ & 3.3 & 1.5 & 4.3 & 4.8 & 0.4 & 1.6 & 0.8 & 3.1 & 2.4 & 4.2 & 1.5 & 2.0 & 3.7 & 2.9 & 0.7 & 0.2 & 5.4 & 0.5 & 1.3 & 0.0 & 1.9 & 1.0 & 0.6 & 2.1 & 0.0 & 5.0 & 10.4 & & \\
\hline 13 Net total social expenditure $[6+12-\mathrm{T} 2]^{\mathrm{b}}$ & 21.0 & 25.8 & 30.5 & 24.0 & 19.3 & 25.3 & 23.4 & 32.7 & 28.4 & 19.7 & 18.0 & 26.4 & 23.4 & 11.8 & 19.6 & 9.0 & 25.3 & 18.8 & 20.5 & 18.6 & 25.0 & 16.7 & 21.8 & 27.8 & 11.3 & 26.9 & 27.5 & 22.2 & $26 \%$ \\
\hline Rank (country ranking, from highest spender to lowest) & 16 & 8 & 2 & 12 & 20 & 9 & 14 & 1 & 3 & 18 & 23 & 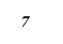 & 13 & 25 & 19 & 27 & 10 & 21 & 17 & 22 & 11 & 24 & 15 & 4 & 26 & 6 & 5 & & \\
\hline Memorandum item & & & & & & & & & & & & & & & & & & & & & & & & & & & & & \\
\hline $\begin{array}{l}\text { TBSPs towards pensions }^{\mathrm{c}} \\
\text { Average indirect tax rate }\end{array}$ & 3.0 & 0.1 & 0.2 & 2.2 & 0.1 & $\cdots$ & 0.1 & 0.0 & 0.9 & 1.2 & 1.4 & 0.0 & 0.8 & & 0.6 & 0.2 & 2.1 & & 0.6 & 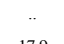 & 0.1 & 0.2 & 0.3 & 0.0 & $\cdots$ & .. & 0.9 & & \\
\hline
\end{tabular}

Notes: a) Numbers in square brackets refer to line numbers in the second column; ".." cell with no information. $\quad$ b) In order to avoid double counting, the value of TBSPs towards "current" private social benefits has been ignored for the calculation of net total social expenditure. c) Because of conceptual issues and gaps in data availability, tax breaks towards old-age pensions are shown in the table as a memorandum item.

Source: see Annex I.2. 
Chart I.11: Using net rather than gross indicators changes the ranking among countries in international comparison of social spending

Rank of countries in terms of gross public and net total social spending-to-GDP ratios, 2007

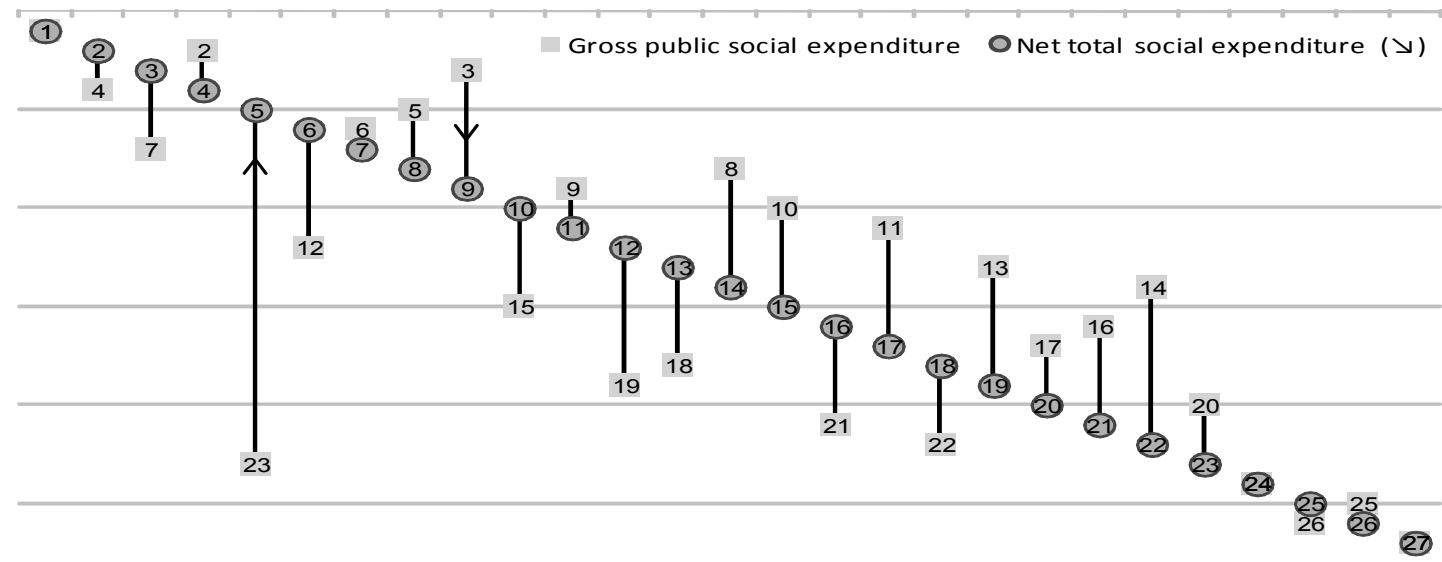

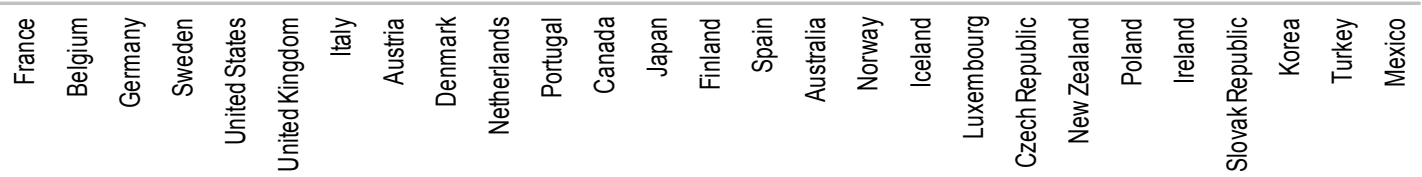

Source: see Annex I.2. 


\section{REFERENCES}

Adema, W. and M. Einerhand (1998), "The Growing Role of Private Social Benefits", Labour Market and Social Policy Occasional Papers, No. 32, OECD, Paris (www.oecd.org/els/workingpapers).

Adema, W. and M. Ladaique (2009) 'How Expensive is the Welfare State? Gross and Net Indicators in the OECD Social Expenditure Database (SOCX)', OECD Social, Employment and Migration Working Papers no. 92, OECD, Paris (www.oecd.org/els/workingpapers).

EUROSTAT (2008), ESSPROS Manual - The European System of integrated Social PROtection Statistics (ESSPROS), Luxembourg (http://epp.eurostat.ec.europa.eu/portal/page/portal/social_protection/publications).

OECD (2010a), Value for Money in Health Spending, Health Policy Studies, OECD, Paris.

OECD (2010b), OECD Revenue Statistics, 1965-2009, OECD, Paris (www.oecd.org/ctp/revenuestats).

OECD (2010c), National Accounts of OECD Countries: Detailed tables, Volume II, OECD, Paris.

OECD (2011a), Economic Outlook 89A, OECD, Paris (www.oecd.org/oecdEconomicOutlook).

OECD (2011b), Pensions at a Glance, OECD, Paris (www.oecd.org/els/social/pensions/PAG).

OECD (2011c), Employment Outlook, OECD, Paris (www.oecd.org/employment/outlook).

OECD (2011d), Economic Crisis and Beyond: Social Policies for the Recovery, Documentation for the 2011 OECD Ministerial Meeting on Social Policy. (www.oecd.org/social/ministerial)

OECD Social Expenditure database - SOCX (www.oecd.org/els/social/expenditure)

OECD Family database (www.oecd.org/els/social/family/database) 


\section{ANNEX I.1: ESTIMATING PUBLIC SOCIAL EXPENDITURE 2008-2012 - SOURCES AND METHODS}

Detailed SOCX data is not available for the period after 2007. However, the public social expenditure series as in SOCX was extended to 2008, 2009 and 2010 using available information on national aggregates in the OECD Economic Outlook and the European Union's Annual macro-economic database (AMECO), or country responses to an OECD questionnaire on social policy experiences during the economic crisis. Based on projections in the OECD Economic Outlook and AMECO, public social spending aggregates were estimated for 2011 and 2012.

Two series were extended from 2007 until 2012 public social expenditure in cash (social transfers) and public social expenditure on services, including health.

A "standard procedure" was applied for the following European OECD countries (Austria, Belgium, the Czech Republic, Denmark, Finland, France, Germany, Greece, Hungary Iceland, Ireland, Italy, Luxembourg, the Netherlands, Norway, Poland, Portugal, the Slovak Republic, Spain, Slovenia, Switzerland, Turkey and the United Kingdom). The procedure involved:

- Social transfers, cash spending : for the years 2008-2012, trends in social security benefits paid by general government (SSPG) as in the database underlying OECD (2011a) Economic Outlook 89A database) were applied to public social expenditure in cash as in 2007. For 2008. 2009 and 2010, (2009 for New Zealand, Switzerland, and Turkey), it concerns spending data as reported by countries; for 2011 and 2012 the estimated SSPG series as in OECD (2011a) was used.

- Services spending : for the years 2008 - 2012, trends in social transfers in kind (series (UCIG0 in the European System of National Accounts ESA 1995) as projected in the AMECO database were applied to public social expenditure on services as in 2007. AMECO is the annual macroeconomic database of the European Commission's Directorate General for Economic and $\begin{array}{lllll}\text { Financial } & \text { Affairs } & \text { (DG } & \text { ECFIN }\end{array}$ (http://ec.europa.eu/economy_finance/db_indicators/ameco/index_en.htm)). For 2008, 2009 and 2010, it concerns spending data as reported by countries; for 2011 and 2012 the estimated UCIG0 series as reported in AMECO was used.

- Public expenditure on Active Labour Market Programmes (ALMPs): For the years 2008 and 2009 data were taken from the LMP database as reported in OECD (2011c), Employment Outlook. From 2010 to 2012 trends in UC1GO series on social transfers in kind (see above) were applied to extend the series on public spending on ALMPs to 2010, 2011 and 2012.

Data on GDP were taken from OECD Economic Outlook database 89A as released May 2011 (www.oecd.org/oecdEconomicOutlook)

For the United States, trends in projections from the Office of Management and Budget were applied at programme level (http://www.whitehouse.gov/omb/budget/Historicals/).

For countries for which either SSPG data (social security benefits paid by general government) or short term economic forecasts from AMECO were not available, results were used from OECD (2011b) 
and country responses to the OECD-ELS questionnaire on the crisis and early recovery (This questionnaire contributed to documentation on for the OECD Ministerial Meeting on Social Policy, 2-3 May 2011, session 1: Economic Crisis and Beyond: Social Policies for the Recovery (OECD, 2011d).

Table AI.1.1 summarises the methodology applied to estimate spending aggregates for public expenditure on cash benefits social services (including health) and ALMPs. Please note that (x) denotes the standard procedure as define above.

Estimates were further refined to obtain extend the series public social spending for the 2008-2012 period for the following four broad social policy areas: Pensions (SOCX categories old Age cash and Survivors); Income support to the working-age population (spending on the following SOCX categories: Incapacity benefits, Family cash benefits, Unemployment and other social policy areas categories; Health; and, other social services (spending on services other than health; includes spending on ALMPs).

Where possible the following method was followed to extend the series as in SOCX:

- Pensions: use the trend in the long-term projections for public pension spending, per cent of GDP as in OECD (2011b), Pensions at a Glance: Retirement-Income Systems in OECD and G20 Countries (www.oecd.org/els/social/pensions/PAG)

- Income support to the working age: Trends in spending as in the social security benefits paid by general government (SSPG) series (OECD Economic Outlook).

- Health: Trend in Health Care expenditures projections (AWG, European Commission Ageing Working Group reference scenario) from the 2009 Ageing Report: Economic and budgetary projections for the EU-27 Member States (2008-2060) EU Ageing report 2009 (http://ec.europa.eu/economy_finance/publications/publication14992_en.pdf). The so-called "AWG reference scenario" was used as "central scenario" when calculating the overall budgetary impact of ageing. It is a combination of a number of factors affecting health care spending and, as such, it is considered by the Ageing Working Group as a plausible scenario for assessing potential future needs for public spending on health care. It incorporates the demographic impact of the changing population structure, moderately positive developments of health status and the strengthened impact of the national income incorporating a number of demand and supply factors pushing expenditure up.

- Other Services: These trends were obtained by deducting projected values on spending on Pensions, Health and Income support to the working-age population from the projected overall public social expenditure trend for 2008-2012.

Table AI.1.2 summarizes the methodology applied for the estimation of public social spending by broad social policy area per country. These estimates are always consistent with series on total public social spending on cash benefits and social services as referred to in Table AI1.1 Please note (x) denotes the procedure as defined in the four bullet points above. 
Table A.I.1.1: Estimation method for public social spending on cash benefits, services and ALMPs, 2008-2012

\begin{tabular}{|c|c|c|c|}
\hline & Cash & Services & $\begin{array}{l}\text { Active Labour Market } \\
\text { Programmes }\end{array}$ \\
\hline Australia & $\mathrm{x}$ & $\begin{array}{l}2005-2008 \text { annual average growth rate from } \\
2009 \text { to } 2012 \text {. }\end{array}$ & $\begin{array}{l}2005-2008 \text { annual average growth } \\
\text { rate for } 2010-2012 \text {. }\end{array}$ \\
\hline Austria & $x$ & $x$ & $x$ \\
\hline Belgium & $\mathrm{x}$ & $\mathrm{x}$ & $x$ \\
\hline Canada & $\mathrm{x}$ & $\begin{array}{l}\text { Data for } 2008-2009 \text { were taken from the } \\
\text { country response to the OECD } \\
\text { questionnaire on the crisis and early } \\
\text { recovery }+2004-2007 \text { annual average growth } \\
\text { rate for } 2010-2012 \text {. } \\
\end{array}$ & $\begin{array}{l}2004-2007 \text { annual average growth } \\
\text { rate for 2010-2012. }\end{array}$ \\
\hline Chile & $\begin{array}{l}\text { Data for 2008-2010 were taken from the } \\
\text { country response to the OECD } \\
\text { questionnaire on the crisis and early } \\
\text { recovery and Central Bank social } \\
\text { expenditure data. }\end{array}$ & $\begin{array}{l}\text { Data for } 2008-2010 \text { were taken from the } \\
\text { country response to the OECD } \\
\text { questionnaire on the crisis and early } \\
\text { recovery and Central Bank social } \\
\text { expenditure data. }\end{array}$ & $\begin{array}{l}\text { Data were taken from the trend in } \\
\text { the OECD Labour Market } \\
\text { programmes Database. }\end{array}$ \\
\hline Czech Republic & $\mathrm{x}$ & $x$ & $x$ \\
\hline Denmark & $\mathrm{x}$ & $\mathrm{x}$ & $x$ \\
\hline Estonia & $\begin{array}{l}\text { Data for } 2008-2010 \text { were taken from the } \\
\text { country response to the OECD } \\
\text { questionnaire on the crisis and early } \\
\text { recovery. For } 2011 \text { and } 2012 \text {, a constant } \\
\text { average growth rate has been considered. } \\
\text { Unemployment cash benefits have been } \\
\text { estimated based on the trend in country } \\
\text { response to the questionnaire on the crisis } \\
\text { and early recovery till } 2012 \text {. }\end{array}$ & $\mathrm{x}$ & $\mathrm{x}$ \\
\hline Finland & $x$ & $x$ & $x$ \\
\hline France & $x$ & $x$ & $x$ \\
\hline Germany & $\mathrm{x}$ & $x$ & $x$ \\
\hline Greece & $x$ & $x$ & $x$ \\
\hline Hungary & $x$ & $x$ & $x$ \\
\hline Iceland & $x$ & $x$ & $x$ \\
\hline Ireland & $\mathrm{x}$ & $x$ & $x$ \\
\hline Israel & $\mathrm{x}$ & $\begin{array}{l}\text { Data for 2008-2011 were taken from the } \\
\text { country response to the OECD } \\
\text { questionnaire on the crisis and early } \\
\text { recovery }+2004-2007 \text { annual average growth } \\
\text { rate for } 2012 \text {. }\end{array}$ & $\mathrm{x}$ \\
\hline Italy & $x$ & $x$ & $x$ \\
\hline Japan & 2008 data were taken from IPSS & 2008 data were taken from IPSS & $\begin{array}{l}2008 \text { data were taken from the } \\
\text { OECD Labour Market } \\
\text { programmes Database. }\end{array}$ \\
\hline Korea & $\begin{array}{l}\text { Data were taken from the country response } \\
\text { to the OECD questionnaire on the crisis and } \\
\text { early recovery for } 2008-2012\end{array}$ & $\begin{array}{l}\text { Data were taken from the country response } \\
\text { to the OECD questionnaire on the crisis and } \\
\text { early recovery for } 2008-2012\end{array}$ & $\begin{array}{l}\text { Data were taken from the country } \\
\text { response to the OECD } \\
\text { questionnaire on the crisis and } \\
\text { early recovery for } 2008-2012\end{array}$ \\
\hline Luxembourg & $x$ & $x$ & $x$ \\
\hline Mexico & $\begin{array}{l}\text { Data were taken from the country response } \\
\text { to the OECD questionnaire on the crisis and } \\
\text { early recovery for } 2008-2011\end{array}$ & $\begin{array}{l}\text { Data were taken from the country response } \\
\text { to the OECD questionnaire on the crisis and } \\
\text { early recovery for } 2008-2011\end{array}$ & $\begin{array}{l}\text { Data were taken from the country } \\
\text { response to the OECD } \\
\text { questionnaire on the crisis and } \\
\text { early recovery for 2008-2011 }\end{array}$ \\
\hline Netherlands & $\mathrm{x}$ & $x$ & $x$ \\
\hline New Zealand & $\begin{array}{l}\text { Data were taken from the country response } \\
\text { to the OECD questionnaire on the crisis and } \\
\text { early recovery for } 2008-2012\end{array}$ & $\begin{array}{l}\text { Data were taken from the country response } \\
\text { to the OECD questionnaire on the crisis and } \\
\text { early recovery for } 2008-2012\end{array}$ & $\begin{array}{l}\text { Data were taken from the country } \\
\text { response to the OECD } \\
\text { questionnaire on the crisis and } \\
\text { early recovery for 2008-2012 }\end{array}$ \\
\hline Norway & $\mathrm{x}$ & $\mathrm{x}$ & $\begin{array}{l}\text { Data were taken from the trend in } \\
\text { Social transfers in kind from } \\
\text { Ameco }\end{array}$ \\
\hline \begin{tabular}{|l|} 
Poland \\
\end{tabular} & $x$ & $x$ & $x$ \\
\hline \begin{tabular}{|l|} 
Portugal \\
\end{tabular} & $x$ & $x$ & $x$ \\
\hline Slovak Republic & $x$ & $x$ & $x$ \\
\hline \begin{tabular}{|l} 
Slovenia \\
\end{tabular} & $x$ & $x$ & $x$ \\
\hline Spain & $x$ & $x$ & $x$ \\
\hline Sweden & $\mathrm{x}$ & $x$ & $x$ \\
\hline Switzerland & $x$ & $x$ & $\begin{array}{l}\text { Data were taken from the trend in } \\
\text { Social transfers in kind from } \\
\text { Ameco }\end{array}$ \\
\hline \begin{tabular}{|l} 
Turkey \\
\end{tabular} & & Not available & \\
\hline United Kingdom & $x$ & $x$ & $x$ \\
\hline United States & $\begin{array}{l}\text { Data were taken from the previsions of the } \\
\text { Office of management and budget. }\end{array}$ & $\begin{array}{l}\text { Data were taken from the previsions of the } \\
\text { Office of management and budget. }\end{array}$ & $\mathrm{x}$ \\
\hline
\end{tabular}

(x) The "standard procedure" has been followed to generate projections based on, for cash spending, trends in the SSPG series in the OECD Economic Outlook, for services, trends in the UCIG0 series on service spending in the AMECO database and for ALMPs, the OECD Labour market programmes database (OECD (2011b), Employment Outlook) and trends in the UCIG0 series on service spending in the AMECO database. 
Table A.I.1.2: Estimation method for public social spending by broad social policy area, 2008-2012

\begin{tabular}{|c|c|c|c|c|}
\hline & Pension & $\begin{array}{l}\text { Income support to the } \\
\text { working age }\end{array}$ & Health & Other services \\
\hline Australia & $x$ & $x$ & $\begin{array}{l}\text { Trend in Social transfers } \\
\text { in kind from Ameco for } \\
2008 \text { and } 2005-2008 \\
\text { annual average growth } \\
\text { rate from } 2009 \text { to } 2012 \text {. }\end{array}$ & $x$ \\
\hline Austria & $x$ & $x$ & $x$ & $x$ \\
\hline Belgium & $x$ & $x$ & $x$ & $x$ \\
\hline Canada & $x$ & $x$ & $\begin{array}{l}\text { Data for } 2008-2009 \text { were } \\
\text { taken from the country } \\
\text { response to the OECD } \\
\text { questionnaire on the } \\
\text { crisis and early recovery } \\
+2004-2007 \text { annual } \\
\text { average growth rate for } \\
2010-2012 \text {. } \\
\text { avalo }\end{array}$ & $x$ \\
\hline Chile & $\begin{array}{l}\text { Data for } 2008-2010 \text { were } \\
\text { taken from the country } \\
\text { response to the OECDD } \\
\text { questionnaire on the } \\
\text { crisis and early recovery } \\
\text { and from Central Bank } \\
\text { social expenditure data. }\end{array}$ & $\begin{array}{l}\text { Data for } 2008-2010 \text { were } \\
\text { taken from the country } \\
\text { response to the OECD } \\
\text { questionnaire on the } \\
\text { crisis and early recovery } \\
\text { and from Central Bank } \\
\text { social expenditure data. }\end{array}$ & $\begin{array}{l}\text { Data for } 2008-2010 \text { were } \\
\text { taken from the country } \\
\text { response to the OECD } \\
\text { questionnaire on the } \\
\text { crisis and early recovery } \\
\text { and from Central Bank } \\
\text { social expenditure data. }\end{array}$ & $x$ \\
\hline Czech Republic & $x$ & $x$ & $\times$ & $x$ \\
\hline \begin{tabular}{|l|} 
Denmark \\
\end{tabular} & $x$ & $x$ & $x$ & $x$ \\
\hline Estonia & $x$ & $\begin{array}{l}\text { Data for } 2008-2010 \text { were } \\
\text { taken from the country } \\
\text { response to the OECD } \\
\text { questionnaire on the } \\
\text { crisis and early recovery } \\
+2004-2007 \text { annual } \\
\text { average growth rate for } \\
2011-2012 \text { except for } \\
\text { unemployment where } \\
\text { trends based on the } \\
\text { country response to the } \\
\text { questionnaire on thr } \\
\text { crisis and early recovery } \\
\text { has been used till } 2012 \text {. }\end{array}$ & $x$ & $x$ \\
\hline Finland & $x$ & $x$ & $x$ & $x$ \\
\hline France & $x$ & $x$ & $x$ & $x$ \\
\hline Germany & $x$ & $x$ & $x$ & $x$ \\
\hline Greece & $x$ & $x$ & $x$ & $x$ \\
\hline Hungary & $x$ & $x$ & $x$ & $x$ \\
\hline Iceland & $\begin{array}{l}\text { Social security benefits } \\
\text { paid by general } \\
\text { government SSPG (from } \\
\text { OECD ECOnomic } \\
\text { Outlook 89A database) }\end{array}$ & $x$ & $\begin{array}{l}\text { Trend in Social transfers } \\
\text { in kind from Ameco }\end{array}$ & $x$ \\
\hline Ireland & $x$ & $x$ & $x$ & $x$ \\
\hline Israel & $\begin{array}{l}\text { Social security benefits } \\
\text { paid by general } \\
\text { government SSPG (from } \\
\text { OECD ECOnomic } \\
\text { Outlook 89A database) } \\
\end{array}$ & $x$ & $\begin{array}{l}\text { OECD Health data for } \\
2008+2004-2007 \\
\text { annual average growth } \\
\text { rate for } 2009-2012 \text {. }\end{array}$ & $x$ \\
\hline Italy & $x$ & $x$ & $x$ & $x$ \\
\hline \begin{tabular}{|l|l} 
Japan \\
\end{tabular} & Not available & & & \\
\hline Korea & $x$ & $\begin{array}{l}\text { Data were taken from } \\
\text { the country response to } \\
\text { the OECD questionnaire } \\
\text { on the crisis and early } \\
\text { recovery for } 2008-2012\end{array}$ & $\begin{array}{l}\text { Data were taken from } \\
\text { the country response to } \\
\text { the OECD questionnaire } \\
\text { on the crisis and early } \\
\text { recovery for } 2008-2012\end{array}$ & $x$ \\
\hline Luxembourg & $x$ & $x$ & 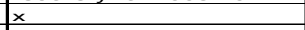 & $x$ \\
\hline Mexico & $\begin{array}{l}\text { Data were taken from } \\
\text { the country response to } \\
\text { the OECD questionnaire } \\
\text { on the crisis and early } \\
\text { recovery for } 2008-2011\end{array}$ & $\begin{array}{l}\text { Data were taken from } \\
\text { the country response to } \\
\text { the OECD questionnaire } \\
\text { on the crisis and early } \\
\text { recovery for } 2008-2011\end{array}$ & $\begin{array}{l}\text { OECD Health data for } \\
2008+D a t a \text { taken from } \\
\text { the country response to } \\
\text { the OECD questionnaire } \\
\text { on the crisis and early } \\
\text { recovery for } 2009-2011\end{array}$ & $x$ \\
\hline \begin{tabular}{|l} 
Netherlands \\
\end{tabular} & $x$ & $x$ & $x$ & $x$ \\
\hline New Zealand & $x$ & $\begin{array}{l}\text { Data were taken from } \\
\text { the country response to } \\
\text { the OECD questionnaire } \\
\text { on the cris is and early } \\
\text { recovery for } 2008-2012 \text {. }\end{array}$ & $\begin{array}{l}\text { Data were taken from } \\
\text { the country response to } \\
\text { the OE CD questionnaire } \\
\text { on the crisis and early } \\
\text { recovery for } 2008+2004 \\
2007 \text { annual average } \\
\text { growth rate for } 2009- \\
2012 \text {. } \\
\end{array}$ & \\
\hline Norway & $x$ & $x$ & $\times$ & $x$ \\
\hline Poland & $x$ & $x$ & $x$ & $x$ \\
\hline Portugal & $x$ & $x$ & $x$ & $x$ \\
\hline Slovak Republic & $x$ & $x$ & $x$ & $x$ \\
\hline Slovenia & $x$ & $x$ & $x$ & $x$ \\
\hline \begin{tabular}{|l} 
Spain \\
\end{tabular} & $x$ & $x$ & $x$ & $x$ \\
\hline Sweden & $x$ & $x$ & $x$ & $x$ \\
\hline Switzerland & $x$ & $x$ & $\begin{array}{l}\text { Trend in Social transfers } \\
\text { in kind from Ameco }\end{array}$ & $x$ \\
\hline \begin{tabular}{|l|} 
Turkey \\
\end{tabular} & Not available & & & \\
\hline United Kingdom & $x$ & $x$ & $x$ & $x$ \\
\hline United States & $\begin{array}{l}\text { Data were taken from } \\
\text { the previsions of the } \\
\text { Office of management } \\
\text { and budget. }\end{array}$ & $\begin{array}{l}\text { Data were taken from } \\
\text { the previsions of the } \\
\text { Office of management } \\
\text { and budget. }\end{array}$ & $\begin{array}{l}\text { Data were taken from } \\
\text { the previsions of the } \\
\text { Office of management } \\
\text { and budget. }\end{array}$ & \\
\hline
\end{tabular}


AMECO explanatory note:

AMECO is the annual macro-economic database of the European Commission's Directorate General for Economic and Financial Affairs (DG ECFIN).

Social transfers in kind; ESA 1995 (UCIG0); ESA 95-code: D.63; Sector affected: General government (S.13); Definition (ESA 1995): 4.104 f; Source: National accounts; Eurostat or National

Social transfers in kind consist of individual goods and services provided as transfers in kind to individual households by government units. They include:

Social benefits in kind (D.631). Social benefits in kind are social transfers in kind intended to relieve the household from the financial burden of social risks or needs. They include the following cases:

- Social security benefits, reimbursements (D.6311). These benefits consist of reimbursement by social security funds of approved expenditures made by households on specific goods or services.

- Other social security benefits in kind (D.6312). These consist of transfers in kind provided to households by government units that are similar in nature to social security benefits in kind but are not provided in the context of social insurance schemes. Social assistance benefits in kind include, if not covered by a social insurance scheme, for instance social housing, dwelling allowances, and reduction of transport prices (provided that there is a social purpose).

Transfers of individual non-market goods or services (D.632). Transfers of individual non-market goods or services consist of goods or services provided to individual households free or at prices which are not economically significant, by non-market producers of government units. They cover for instance education and cultural services.

Social transfers in kind are equal to the individual consumption expenditure of general government; variable UCIG0 is therefore conceptually identical with variable UCIG ('Individual consumption of general government at current prices'). Differences between UCIG0 and UCIG which may occur are due to different transmission deadlines of the underlying sources. UCIG0 is based on table 2 of the ESA 1995 transmission programme ('Main aggregates of general government') which has a deadline of $t+8$ months. Table 1 of the ESA 1995 transmission programme ('Main aggregates'), which is used for variable UCIG, has to be provided after $\mathrm{t}+70$ days. 
DELSA/ELSA/WD/SEM(2011)9

Table A.I.1.3: Public social expenditures as \% GDP, 1980 - 2012, estimated for 2008 to 2012

\begin{tabular}{|c|c|c|c|c|c|c|c|c|c|c|c|c|c|c|c|c|c|c|c|c|c|c|c|c|c|c|c|c|c|c|c|c|c|}
\hline & J & & 82 & 83 & 84 & 885 & 1986 & 1987 & 1988 & 1989 & 1990 & 1991 & 1992 & 1993 & 1994 & 1995 & 1996 & 1997 & 71998 & 81999 & 2000 & 2001 & 2002 & 2003 & 2004 & 42005 & 2006 & 200 & 2008 & 20 & 20 & 20 & 20 \\
\hline tralia & 3 & 0.1 & 11.1 & 11.6 & 12.0 & 12.1 & 12.0 & 12.1 & 111.5 & 11.6 & 13.1 & 14.3 & 15.2 & 15.5 & 15.3 & 16.2 & 16.2 & 16.0 & 16.7 & 716.5 & 517.3 & $\begin{array}{l}316.8 \\
\end{array}$ & 17.0 & 17.2 & 171 & 116.5 & 16.1 & 16.0 & 16.5 & 18.0 & 16.6 & 16.4 & 1 \\
\hline stria & 22.4 & $\mathrm{~m}$ & $\mathrm{~m}$ & $\mathrm{~m}$ & $\mathrm{~m}$ & 23.7 & $\mathrm{~m}$ & $\mathrm{~m}$ & $\mathrm{~m}$ & $\mathrm{~m}$ & 23.8 & 24.1 & 24.8 & 26.2 & 27.0 & 26.6 & 26.7 & 26.8 & B 26.6 & $6 \quad 27.0$ & 26.7 & $7 \quad 27.0$ & 27.3 & 27.9 & 27.7 & $\begin{array}{ll}7 & 27.4\end{array}$ & 27.0 & 26.4 & 26.7 & 29.1 & 28.9 & 28.3 & 28.1 \\
\hline Igium & 23.5 & 25.1 & 25.7 & 26.0 & 25.2 & 26.0 & 25.9 & 25.6 & 25.5 & 24.6 & 24.9 & 25.7 & 25.8 & 26.9 & 26.3 & 26.3 & 26.8 & 25.7 & 26.0 & 25.9 & 25.4 & $4 \quad 25.8$ & 26.2 & 26.3 & 26.5 & $5 \quad 26.4$ & 26.4 & 26.3 & 7.4 & 29.7 & 29.4 & 28.9 & 28.6 \\
\hline nada & .7 & 14.1 & 16.5 & 16.7 & 16.5 & 17.0 & 16.9 & 16.6 & 16.4 & 16.7 & 18.1 & 20.3 & 21.0 & 20.9 & 19.9 & 18.9 & 18.1 & 17.4 & $4 \quad 17.7$ & $7 \quad 16.7$ & $7 \quad 16.5$ & $5 \quad 17.0$ & 17.1 & 17.2 & 17.1 & 117.0 & 16.9 & 16.9 & 17.7 & 19.6 & 19.3 & 19.3 & 19.3 \\
\hline Chile & $\mathrm{m}$ & $\mathrm{m}$ & $\mathrm{m}$ & $\mathrm{m}$ & $\mathrm{m}$ & $\mathrm{m}$ & $\mathrm{m}$ & $\mathrm{m}$ & $\mathrm{m}$ & $\mathrm{m}$ & $\mathrm{m}$ & $\mathrm{m}$ & $\mathrm{m}$ & $\mathrm{m}$ & $\mathrm{m}$ & 11.4 & 12.2 & 11.9 & $\begin{array}{ll}9 & 12.2\end{array}$ & 213.1 & 113.2 & 213.4 & 13.4 & 12.8 & 11.7 & $\begin{array}{lll}7 & 11.2\end{array}$ & 10.5 & 10.6 & 10.6 & 12.5 & 11.6 & m & $m$ \\
\hline ech & $\mathrm{m}$ & $\mathrm{m}$ & $\mathrm{m}$ & $\mathrm{m}$ & $\mathrm{m}$ & $\mathrm{m}$ & $\mathrm{m}$ & $\mathrm{m}$ & $\mathrm{m}$ & $\mathrm{m}$ & 16.0 & 17.3 & 17.6 & 18.1 & 18.1 & 18.2 & 18.0 & 18.8 & 319.0 & 19.5 & $\begin{array}{ll}5 & 19.8\end{array}$ & $\begin{array}{ll}3 & 19.8\end{array}$ & 20.6 & 20.7 & 19.7 & $\begin{array}{ll}7 & 19.5\end{array}$ & 19.1 & 18.8 & 18.7 & 20.3 & 20.4 & 20.6 & 20.4 \\
\hline mar & 24.8 & 24.8 & 24.8 & 25.0 & 23.8 & 23.2 & 23.1 & 23.8 & 25.1 & 25.3 & 25.1 & 25.9 & 26.4 & 28.1 & 29.4 & 28.9 & 28.2 & 27.2 & 26.5 & $5 \quad 26.4$ & $\begin{array}{l}4 \\
+\end{array}$ & 726.1 & 26.6 & 27.9 & 27.7 & $\begin{array}{ll}7 & 27.2\end{array}$ & 26.6 & 26.0 & 26.6 & 30.2 & 30.1 & 29.9 & 29.5 \\
\hline Ullic & $\mathrm{m}$ & $\mathrm{m}$ & $\mathrm{m}$ & $\mathrm{m}$ & $\mathrm{m}$ & $\mathrm{m}$ & $\mathrm{m}$ & $\mathrm{m}$ & $\mathrm{m}$ & $\mathrm{m}$ & $\mathrm{m}$ & $\mathrm{m}$ & $\mathrm{m}$ & $\mathrm{m}$ & $\mathrm{m}$ & $\mathrm{m}$ & $\mathrm{m}$ & $\mathrm{m}$ & $\mathrm{m}$ & $\mathrm{m}$ & 14.1 & $1 \quad 13.2$ & $2 \quad 13.0$ & 13.1 & 13.5 & $\begin{array}{ll}5 & 13.2\end{array}$ & 12.8 & 13.0 & 0.6 & 19.1 & 19.7 & 18.3 & 1.3 \\
\hline ind & 18.1 & 18.5 & 19.5 & 20.5 & 21.5 & 22.6 & 23.0 & 23.6 & 23.0 & 22.7 & 24.3 & 29.4 & 33.4 & 33.4 & 32.8 & 30.9 & 30.8 & 28.6 & 526.4 & $\begin{array}{l}4 \\
4\end{array}$ & $\begin{array}{ll}B & 24.3\end{array}$ & $\begin{array}{ll}3 & 24.3\end{array}$ & $\begin{array}{l}325.0 \\
\end{array}$ & 5.9 & 26.0 & $0 \quad 26.1$ & 25.9 & 24.9 & 0.1 & 29.6 & 29.1 & 28.4 & 28.0 \\
\hline nce & 8 & 21.8 & 22.3 & 22.6 & 22.8 & 26.0 & 25.8 & 25.9 & 25.7 & 24.8 & 24.9 & 25.5 & 26.3 & 27.8 & 27.8 & 28.5 & 28.8 & 28.6 & 28.8 & 328.8 & 7.7 & 7.7 & $7 \quad 28.4$ & 28.9 & 29.0 & 029.0 & 28.6 & 28.4 & 8.6 & 30.7 & 31.0 & 30.4 & 29.9 \\
\hline $\mathrm{ma}$ & 22.1 & 22.8 & 22.8 & 22.5 & 22.2 & 22.5 & 22.5 & 23.0 & 23.0 & 21.9 & 21.7 & 23.7 & 25.6 & 26.3 & 26.3 & 26.8 & 7.4 & 26.7 & 726.6 & $6 \quad 26.6$ & 626.6 & $\begin{array}{ll}6 & 26.7\end{array}$ & $7 \quad 27.4$ & 27.7 & 27.1 & $\begin{array}{ll}127.2\end{array}$ & 26.1 & 25.2 & 5.2 & 27.6 & 27.3 & 26.4 & 25.8 \\
\hline ect & 10.2 & 12.3 & 14.5 & 15.1 & 15.4 & 16.0 & 15.8 & 15.8 & 14.6 & 15.5 & 16.5 & 15.9 & 16.1 & 17.0 & 17.0 & 17.3 & 17.9 & 17.9 & $\begin{array}{ll}9 & 18.6\end{array}$ & $\begin{array}{ll}6 & 19.2\end{array}$ & 19.2 & 20.6 & $\begin{array}{ll}620.1 \\
\end{array}$ & 19.8 & 19.9 & $\begin{array}{ll}9 & 21.0\end{array}$ & 21.3 & 21.3 & 2.7 & 24.6 & 23.2 & 23.4 & 23.1 \\
\hline an & $\mathrm{m}$ & $\mathrm{m}$ & $\mathrm{m}$ & $\mathrm{m}$ & $\mathrm{m}$ & $\mathrm{m}$ & $\mathrm{m}$ & $\mathrm{m}$ & $\mathrm{m}$ & $\mathrm{m}$ & $\mathrm{m}$ & $\mathrm{m}$ & $\mathrm{m}$ & $\mathrm{m}$ & $\mathrm{m}$ & $\mathrm{m}$ & $\mathrm{m}$ & $\mathrm{m}$ & $\mathrm{m}$ & 21.2 & 20.3 & $\begin{array}{ll}3 & 20.1\end{array}$ & 121.3 & 22.4 & 21.6 & $\begin{array}{ll}622.6 \\
\end{array}$ & 22.9 & 22.9 & 23.5 & 24.4 & 23.5 & 23.1 & 22.1 \\
\hline anc & $\mathrm{m}$ & $\mathrm{m}$ & $\mathrm{m}$ & $\mathrm{m}$ & $\mathrm{m}$ & $\mathrm{m}$ & $\mathrm{m}$ & $\mathrm{m}$ & $\mathrm{m}$ & $\mathrm{m}$ & 13.7 & 14.2 & 14.7 & 15.1 & 14.9 & 15.2 & 14.9 & 14.6 & $\begin{array}{ll}6 & 14.8\end{array}$ & $\begin{array}{ll}B & 15.4\end{array}$ & $4 \quad 15.2$ & 5.3 & $\begin{array}{ll}3 & 16.8\end{array}$ & 17.7 & 17.4 & $\begin{array}{ll}4 & 16.3\end{array}$ & 15.9 & 14 & 75.7 & 17. & 15. & 74.8 & 14.0 \\
\hline and & 6.7 & 16.7 & 17.4 & 17.5 & 17.0 & 21.3 & 21.5 & 20.7 & 19.4 & 17.8 & 14.9 & 15.7 & 16.4 & 16.4 & 16.1 & 15.7 & 4.8 & 14.0 & 13.0 & 4.1 & 113.3 & $\begin{array}{ll}3 & 14.3\end{array}$ & 315.2 & 15.6 & 16.0 & $0 \quad 15.8$ & 15.8 & 16 & 78.8 & 22. & 22 & 21. & 19.8 \\
\hline & $\mathrm{m}$ & $\mathrm{m}$ & $\mathrm{m}$ & $\mathrm{m}$ & $\mathrm{m}$ & $\mathrm{m}$ & $\mathrm{m}$ & $\mathrm{m}$ & $\mathrm{m}$ & $\mathrm{m}$ & $\mathrm{m}$ & $\mathrm{m}$ & $\mathrm{m}$ & $\mathrm{m}$ & $\mathrm{m}$ & 16.7 & 7.2 & 17.5 & 517.3 & $\begin{array}{ll}3 & 17.3\end{array}$ & $\begin{array}{ll}3 & 17.1\end{array}$ & 118.5 & $\begin{array}{ll}5 & 18.8\end{array}$ & 18.3 & 17.1 & 116.5 & 15.9 & 15 & 15.8 & 16.2 & 16. & 16.1 & 15.7 \\
\hline taly & 8.0 & 19.4 & 19.9 & 20.9 & 20.5 & 20.8 & 20.8 & 21.0 & 21.0 & 21.2 & 19.9 & 20.3 & 20.9 & 21.1 & 20.8 & 19.9 & 22.0 & 22.7 & 722.9 & $\begin{array}{ll}9 & 23.3\end{array}$ & $\begin{array}{ll}3 & 23.3\end{array}$ & $\begin{array}{ll}3 & 23.5\end{array}$ & $5 \quad 24.0$ & 24.4 & 24.7 & $\begin{array}{ll}7 & 25.0\end{array}$ & 25.1 & 24.9 & 25.6 & 27.5 & 27.5 & 27.0 & 26.4 \\
\hline an & 10.4 & 10.8 & 11.2 & 11.5 & 11.3 & 11.2 & 11.6 & 11.7 & 11.4 & 11.1 & 11.3 & 11.5 & 12.1 & 12.8 & 13.4 & 14.3 & 14.5 & 14.7 & $7 \quad 15.5$ & 516.1 & 116.5 & 517.4 & $\begin{array}{l}4 \quad 17.8 \\
\end{array}$ & 18.1 & 18.2 & 218.6 & 18.4 & 18.7 & 20.0 & $m$ & $m$ & $m$ & $m$ \\
\hline $\mathrm{X} \mid \mathrm{CC}$ & $\mathrm{m}$ & $\mathrm{m}$ & $\mathrm{m}$ & $\mathrm{m}$ & $\mathrm{m}$ & 1.7 & 1.7 & 1.7 & 1.9 & 2.5 & 3.3 & 3.7 & 4.0 & 4.3 & 4.7 & 4.3 & 4.1 & 4.1 & 4.6 & 5.3 & $\begin{array}{l}3 \\
5.3\end{array}$ & $\begin{array}{ll}3 & 6.0\end{array}$ & 6.3 & 6.7 & 6.6 & 6.8 & 7.0 & 7.2 & 8.4 & 8.8 & 8.2 & 8.5 & $m$ \\
\hline herlar & 8 & 25.5 & 27.0 & 27.4 & 26.0 & 25.3 & 24.7 & 24.7 & 24.3 & 24.0 & 25.6 & 25.5 & 26.0 & 26.1 & 24.7 & 23.8 & 22.6 & 21.8 & $\begin{array}{ll}B & 21.4\end{array}$ & $4 \quad 20.5$ & 519.8 & $\begin{array}{ll}B & 19.7\end{array}$ & $7 \quad 20.5$ & 21.2 & 21.1 & 120.7 & 20.3 & 20. & 0.2 & 22.5 & 22.6 & 22.2 & 21.5 \\
\hline 70 & 0 & 17.2 & 18.1 & 17.8 & 17.2 & 17.7 & 17.6 & 18.4 & 19.8 & 21.1 & 21.5 & 21.9 & 21.8 & 20.0 & 19.2 & 18.7 & 18.6 & 19.6 & 0.1 & 119.4 & $4 \quad 19.1$ & $1 \quad 18.4$ & $4 \quad 18.5$ & 18.0 & 17.7 & $\begin{array}{ll}7 & 18.1\end{array}$ & 19.0 & 18. & 20.1 & 21.4 & 21.8 & 22.1 & 21.8 \\
\hline way & 9 & $\mathrm{~m}$ & $\mathrm{~m}$ & $\mathrm{~m}$ & $\mathrm{~m}$ & 17.8 & $\mathrm{~m}$ & $\mathrm{~m}$ & 20.9 & 21.9 & 22.3 & 23.3 & 24.2 & 24.1 & 23.9 & 23.3 & 22.5 & 22.0 & 23.6 & $6 \quad 23.6$ & b 21.3 & $\begin{array}{ll}3 & 22.2\end{array}$ & $2 \quad 23.7$ & 24.6 & 23.3 & $\begin{array}{ll}3 & 21.7\end{array}$ & 20.4 & 20.8 & 20.6 & 24.0 & 24.0 & 22.7 & 22.4 \\
\hline & $\mathrm{m}$ & $\mathrm{m}$ & $\mathrm{m}$ & $\mathrm{m}$ & $\mathrm{m}$ & $\mathrm{m}$ & $\mathrm{m}$ & $\mathrm{m}$ & $\mathrm{m}$ & $\mathrm{m}$ & 14.9 & 21.2 & 24.9 & 24.4 & 23.2 & 22.6 & 2.8 & 22.2 & 1.4 & 1.6 & 0.5 & 1.9 & 922.3 & 22.3 & 21.4 & 421.0 & 20.8 & 19.8 & 9.9 & 21.4 & 21.8 & 21.4 & 21.1 \\
\hline rtugal & 9.9 & 10.7 & 10.0 & 10.1 & 10.0 & 10.1 & 10.7 & 11.0 & 11.1 & 10.7 & 12.5 & 13.4 & 14.1 & 15.5 & 15.7 & 16.5 & 17.1 & 16.9 & 9 17.3 & $\begin{array}{ll}3 & 17.7\end{array}$ & $7 \quad 18.9$ & $\begin{array}{ll}9 & 19.2\end{array}$ & 220.5 & 22.2 & 22.4 & $\begin{array}{l}422.9 \\
\end{array}$ & 22.9 & 22.5 & 23.0 & 25.9 & 26.1 & 25.3 & 25.4 \\
\hline Slovak & $\mathrm{m}$ & $\mathrm{m}$ & $\mathrm{m}$ & $\mathrm{m}$ & $\mathrm{m}$ & $\mathrm{m}$ & $\mathrm{m}$ & $\mathrm{m}$ & $\mathrm{m}$ & $\mathrm{m}$ & $\mathrm{m}$ & $\mathrm{m}$ & $\mathrm{m}$ & $\mathrm{m}$ & $\mathrm{m}$ & 18.8 & 18.4 & 18.0 & 18.1 & 118.6 & 17.9 & 9 17.6 & $\begin{array}{ll}6 & 17.7\end{array}$ & 17.0 & 16.5 & $\begin{array}{ll}5 & 16.3\end{array}$ & 16.0 & 15.7 & 15.8 & 18.5 & 18.2 & 17.6 & 17.0 \\
\hline Slovenia & $\mathrm{m}$ & $\mathrm{m}$ & $\mathrm{m}$ & $\mathrm{m}$ & $\mathrm{m}$ & $\mathrm{m}$ & $\mathrm{m}$ & $\mathrm{m}$ & $\mathrm{m}$ & $\mathrm{m}$ & $\mathrm{m}$ & $\mathrm{m}$ & $\mathrm{m}$ & $\mathrm{m}$ & $\mathrm{m}$ & $\mathrm{m}$ & 22.4 & 22.8 & $\begin{array}{ll}B & 22.9\end{array}$ & $\begin{array}{ll}92.9\end{array}$ & $\begin{array}{ll}9 & 22.9\end{array}$ & $\begin{array}{ll}9 & 23.0\end{array}$ & $0 \quad 23.1$ & 22.6 & 22.2 & $2 \quad 21.9$ & 21.5 & 20.3 & 0.9 & 23.6 & 23.9 & 24.0 & 23.7 \\
\hline & 5 & 16.7 & 16.7 & 17.4 & 17.2 & 17.8 & 17.5 & 17.4 & 17.9 & 18.1 & 19.9 & 20.7 & 21.8 & 23.1 & 22.0 & 21.4 & 21.3 & 20.7 & 720.6 & $6 \quad 20.4$ & $4 \quad 20.4$ & $4 \quad 20.1$ & 120.4 & 21.0 & 21.2 & $2 \quad 21.4$ & 21.4 & 21.6 & 23.1 & 26.3 & 26.7 & 25.9 & 25.3 \\
\hline & 2 & 28.0 & 27.9 & 28.2 & 27.4 & 29.5 & 29.5 & 29.6 & 30.0 & 29.3 & 30.2 & 31.8 & 34.7 & 35.7 & 34.4 & 32.0 & 31.6 & 30.4 & 430.2 & $2 \quad 29.7$ & 728.4 & $4 \quad 28.7$ & $\begin{array}{ll}7 \quad 29.4\end{array}$ & 30.1 & 29.5 & $5 \quad 29.1$ & 28.4 & 27. & 27.3 & 29.6 & 28.2 & 27.2 & 26.5 \\
\hline WEII & 13.8 & 13.5 & 14.3 & 14.7 & 14.8 & 14.7 & 14.8 & 15.0 & 15.0 & 14.7 & 13.5 & 14.5 & 16.0 & 17.4 & 17.3 & 17.5 & 18.0 & 18.6 & 18.7 & $7 \quad 18.5$ & $\begin{array}{ll}517.8 \\
\end{array}$ & $\begin{array}{ll}3 & 18.3\end{array}$ & $\begin{array}{ll}3 & 19.1\end{array}$ & 20.2 & 20.2 & $2 \quad 20.2$ & 19.2 & 18. & 18.1 & 19.6 & 19.6 & 19.1 & 18.5 \\
\hline key & 3.2 & 3.3 & 3.6 & 3.9 & 3.4 & 3.1 & 3.3 & 3.4 & 4.0 & 4.7 & 5.7 & 6.1 & 6.3 & 6.2 & 5.9 & 5.6 & 7.2 & 8.0 & 8.3 & 9.8 & 9.8 & 9.8 & 9.8 & 9.8 & 9.9 & 9.9 & 0.0 & 10.5 & $m$ & m & $m$ & $m$ & $m$ \\
\hline Jnited & 16.5 & 18.0 & 18.5 & 19.3 & 19.3 & 19.4 & 19.5 & 18.8 & 17.5 & 16.9 & 16.7 & 18.2 & 19.9 & 20.5 & 20.1 & 19.9 & 19.6 & 18.7 & $7 \quad 18.9$ & 918.6 & 18.6 & 619.3 & $\begin{array}{ll}3 & 19.4\end{array}$ & 19.8 & 20.5 & $5 \quad 20.6$ & 20.4 & 20.5 & 21.5 & 24.3 & 24.4 & 23.7 & 22.9 \\
\hline ted St & 13.2 & 13.5 & 13.9 & 14.1 & 13.1 & 13.1 & 13.2 & 13.1 & 13.1 & 13.1 & 13.5 & 14.4 & 15.1 & 15.4 & 15.3 & 15.4 & 15.2 & 14.8 & $\begin{array}{ll}B & 14.8\end{array}$ & $\begin{array}{ll}B & 14.5\end{array}$ & 514.5 & $5 \quad 15.3$ & $\begin{array}{ll}3 & 15.9\end{array}$ & 16.0 & 15.9 & $\begin{array}{l}9 \\
9\end{array}$ & 16.0 & 16.2 & 16.8 & 19.5 & 20.4 & 20.3 & 19.5 \\
\hline OECD & 15.7 & 16.3 & 16.8 & 17.1 & 16.8 & 17.4 & 17.4 & 17.5 & 17.4 & 17.3 & 17.7 & 18.6 & 19.5 & 20.0 & 19.7 & 19.5 & 19.6 & 19.3 & 319.3 & $\begin{array}{ll}3 & 19.3 \\
\end{array}$ & 19.0 & 19.3 & $\begin{array}{ll}3 & 19.7 \\
\end{array}$ & 20.1 & 19.9 & $\begin{array}{ll}9 & 19.8 \\
\end{array}$ & 19.5 & 19.3 & 20.2 & 22.5 & 22.2 & 22.1 & 22.1 \\
\hline
\end{tabular}

Note: Social spending aggregates based on detailed data for 1980-2007; aggregate spending estimates for 2008-2012. 


\section{DELSA/ELSA/WD/SEM(2011)9}

Table A.I.1.4a: Public social expenditures on pension as \% GDP, 1980 - 2012, estimated for 2008 to 2012

\begin{tabular}{|c|c|c|c|c|c|c|c|c|c|c|c|c|c|c|c|c|c|c|c|c|c|c|c|c|c|c|c|c|c|c|c|c|c|}
\hline & 1980 & 1981 & 1982 & 1983 & 1984 & 1985 & 1986 & 1987 & 1988 & 1989 & 1990 & 1991 & 1992 & 1993 & 1994 & 1995 & 1996 & 1997 & 1998 & 1999 & 2000 & 2001 & 2002 & 2003 & 2004 & 2005 & 2006 & 2007 & 2008 & 2009 & 2010 & 201 & 2012 \\
\hline Australia & 3.5 & 3.5 & 3.6 & 3.6 & 3.5 & 3.3 & 3.3 & 3.2 & 2.8 & 2.8 & 3.0 & 3.1 & 3.1 & 3.3 & 3.1 & 3.6 & 3.6 & 3.5 & 3.4 & 3.2 & 3.8 & 3.3 & 3.3 & 3.4 & 3.5 & 3.3 & 3.3 & 3.4 & 3.4 & 3.4 & 3.4 & 3.4 & 3.4 \\
\hline ustria & 10.4 & $\mathrm{~m}$ & $\mathrm{~m}$ & $\mathrm{~m}$ & $\mathrm{~m}$ & 11.4 & $\mathrm{~m}$ & $\mathrm{~m}$ & $\mathrm{~m}$ & $\mathrm{~m}$ & 11.4 & 11.5 & 11.5 & 12.1 & 12.3 & 12.3 & 12.4 & 12.4 & 12.3 & 12.4 & 12.3 & 12.5 & 12.6 & 12.8 & 12.7 & 12.5 & 12.4 & 12.3 & 12.2 & 12.2 & 12.2 & 12.2 & 12.2 \\
\hline Belgium & 8.9 & 9.4 & 9.5 & 9.7 & 9.4 & 9.3 & 9.4 & 9.3 & 9.5 & 9.2 & 9.1 & 9.4 & 9.6 & 9.8 & 9.6 & 9.4 & 9.5 & 9.3 & 9.2 & 9.1 & 8.9 & 9.0 & 9.0 & 9.1 & 9.0 & 9.0 & 8.9 & 8.9 & 8.9 & 9.0 & 9.1 & 9.2 & 9.3 \\
\hline Canada & 3.0 & 3.1 & 3.4 & 3.5 & 3.6 & 3.7 & 3.9 & 4.0 & 3.9 & 4.0 & 4.2 & 4.6 & 4.7 & 4.8 & 4.7 & 4.7 & 4.7 & 4.7 & 4.6 & 4.4 & 4.3 & 4.4 & 4.4 & 4.3 & 4.2 & 4.2 & 4.2 & 4.2 & 4.3 & 4.4 & 4.6 & 4.6 & 4.7 \\
\hline Chile & $\mathrm{m}$ & $\mathrm{m}$ & $\mathrm{m}$ & $\mathrm{m}$ & $\mathrm{m}$ & $\mathrm{m}$ & $\mathrm{m}$ & 9.9 & 8.7 & 8.3 & 8.3 & 8.2 & 7.8 & 7.8 & 7.6 & 6.9 & 7.4 & 7.1 & 7.1 & 7.6 & 7.5 & 7.5 & 7.4 & 7.0 & 6.4 & 5.9 & 5.4 & 5.2 & 5.1 & 5.4 & 5.2 & $m$ & $m$ \\
\hline Czech Repu & $\mathrm{m}$ & $\mathrm{m}$ & $\mathrm{m}$ & $\mathrm{m}$ & $\mathrm{m}$ & $\mathrm{m}$ & $\mathrm{m}$ & $\mathrm{m}$ & $\mathrm{m}$ & $\mathrm{m}$ & 6.1 & 6.6 & 6.7 & 6.3 & 6.0 & 6.3 & 6.5 & 7.3 & 7.3 & 7.4 & 7.5 & 7.4 & 7.6 & 7.5 & 7.1 & 7.3 & 7.2 & 7.4 & 7.2 & 7.0 & 6.8 & 6.7 & 6.7 \\
\hline Denmark & 4.8 & 4.8 & 4.7 & 4.7 & 4.6 & 4.7 & 4.5 & 4.6 & 4.8 & 5.1 & 5.1 & 5.2 & 5.2 & 5.3 & 6.3 & 6.2 & 6.1 & 5.8 & 5.7 & 5.6 & 5.3 & 5.3 & 5.3 & 5.4 & 5.3 & 5.4 & 5.5 & 5.5 & 5.6 & 5.7 & 5.7 & 5.8 & 5.9 \\
\hline tonia & $\mathrm{m}$ & $\mathrm{m}$ & $\mathrm{m}$ & $\mathrm{m}$ & $\mathrm{m}$ & $\mathrm{m}$ & $\mathrm{m}$ & $\mathrm{m}$ & $\mathrm{m}$ & $\mathrm{m}$ & $\mathrm{m}$ & $\mathrm{m}$ & $\mathrm{m}$ & $\mathrm{m}$ & $\mathrm{m}$ & $\mathrm{m}$ & $\mathrm{m}$ & $\mathrm{m}$ & $\mathrm{m}$ & $\mathrm{m}$ & 6.0 & 5.5 & 5.5 & 5.4 & 5.5 & 5.3 & 5.3 & 5.2 & 5.5 & 5.7 & 6.0 & 5.9 & 5.9 \\
\hline nland & 5.5 & 5.7 & 5.9 & 6.4 & 7.2 & 7.4 & 7.3 & 7.6 & 7.2 & 7.0 & 7.3 & 8.5 & 9.5 & 9.4 & 9.1 & 8.8 & 9.0 & 8.4 & 7.9 & 7.9 & 7.7 & 7.8 & 8.1 & 8.4 & 8.3 & 8.4 & 8.5 & 8.3 & 8.5 & 8.6 & 8.8 & 9.0 & 9.2 \\
\hline ance & 9.4 & 9.7 & 9.9 & 10.1 & 10.3 & 10.5 & 10.4 & 10.4 & 10.5 & 10.0 & 10.6 & 10.9 & 11.2 & 11.8 & 11.9 & 12.0 & 12.2 & 12.2 & 12.0 & 12.1 & 11.8 & 11.8 & 11.9 & 12.1 & 12.2 & 12.3 & 12.4 & 12.5 & 12.6 & 12.8 & 13.0 & 13.0 & 13.0 \\
\hline ermany & 10.4 & 10.4 & 10.5 & 10.5 & 10.4 & 10.3 & 10.1 & 10.3 & 10.2 & 9.9 & 9.7 & 9.5 & 9.8 & 10.1 & 10.3 & 10.7 & 10.9 & 11.0 & 11.1 & 11.2 & 11.2 & 11.3 & 11.5 & 11.7 & 11.6 & 11.5 & 11.1 & 10.7 & 10.6 & 10.5 & 10.5 & 10.5 & 10.4 \\
\hline ece & 5.4 & 5.9 & 7.5 & 7.6 & 8.2 & 8.6 & 8.7 & 9.0 & 8.8 & 9.2 & 9.9 & 9.3 & 9.2 & 9.5 & 9.4 & 9.6 & 10.0 & 10.1 & 10.8 & 11.0 & 10.7 & 11.5 & 11.2 & 11.0 & 11.1 & 11.7 & 11.8 & 11.9 & 11.9 & 11.8 & 11.8 & 11.9 & 12.0 \\
\hline ngary & $\mathrm{m}$ & $\mathrm{m}$ & $\mathrm{m}$ & $\mathrm{m}$ & $\mathrm{m}$ & $\mathrm{m}$ & $\mathrm{m}$ & $\mathrm{m}$ & $\mathrm{m}$ & $\mathrm{m}$ & $\mathrm{m}$ & $\mathrm{m}$ & $\mathrm{m}$ & $\mathrm{m}$ & $\mathrm{m}$ & $\mathrm{m}$ & $\mathrm{m}$ & $\mathrm{m}$ & $\mathrm{m}$ & 7.7 & 7.4 & 7.5 & 8.0 & 8.0 & 8.0 & 8.6 & 8.7 & 9.1 & 9.2 & 9.4 & 9.5 & 9.4 & 9.3 \\
\hline land & $\mathrm{m}$ & $\mathrm{m}$ & $\mathrm{m}$ & $\mathrm{m}$ & $\mathrm{m}$ & $\mathrm{m}$ & $\mathrm{m}$ & $\mathrm{m}$ & $\mathrm{m}$ & $\mathrm{m}$ & 2.2 & 2.3 & 2.4 & 2.5 & 2.4 & 2.4 & 2.3 & 2.3 & 2.2 & 2.2 & 2.2 & 2.1 & 2.1 & 2.3 & 2.1 & 2.0 & 1.8 & 1.9 & 2.0 & 2.7 & 2.6 & 2.4 & 2.3 \\
\hline eland & 5.2 & 5.4 & 5.8 & 5.9 & 5.7 & 5.6 & 5.6 & 5.5 & 5.3 & 4.9 & 3.9 & 4.0 & 4.0 & 3.9 & 3.8 & 3.5 & 3.2 & 2.9 & 2.7 & 3.2 & 3.1 & 3.2 & 3.3 & 3.3 & 3.4 & 3.4 & 3.4 & 3.6 & 3.6 & 3.6 & 3.7 & 3.7 & 3.7 \\
\hline rae & $\mathrm{m}$ & $\mathrm{m}$ & $\mathrm{m}$ & m & $\mathrm{m}$ & $\mathrm{m}$ & $\mathrm{m}$ & $\mathrm{m}$ & $\mathrm{m}$ & $\mathrm{m}$ & $\mathrm{m}$ & $\mathrm{m}$ & $\mathrm{m}$ & $\mathrm{m}$ & $\mathrm{m}$ & 4.7 & 4.8 & 5.0 & 5.0 & 4.9 & 4.9 & 5.3 & 5.4 & 5.5 & 5.4 & 5.1 & 4.9 & 4.8 & 4.9 & 5.1 & 0.1 & 5.1 & 5.0 \\
\hline Italy & 8.9 & 9.8 & 10.2 & 10.9 & 10.8 & 11.1 & 11.4 & 11.4 & 11.4 & 11.6 & 10.1 & 10.4 & 11.0 & 11.4 & 11.6 & 11.3 & 13.3 & 13.8 & 13.7 & 13.9 & 13.6 & 13.4 & 13.6 & 13.8 & 13.8 & 14.0 & 13.9 & 14.1 & \begin{tabular}{|l|}
14.1 \\
\end{tabular} & 14.1 & 14.1 & 14.1 & 14.1 \\
\hline Japan & 3.9 & 4.2 & 4.5 & 4.6 & 4.7 & 4.8 & 5.0 & 5.1 & 5.0 & 4.9 & 4.9 & 4.9 & 5.2 & 5.5 & 5.8 & 6.1 & 6.3 & 6.5 & 6.9 & 7.2 & 7.4 & 7.7 & 8.1 & 8.3 & 8.5 & 8.7 & 8.7 & 8.8 & 9.3 & $m$ & $m$ & $m$ & $m$ \\
\hline xemb & 9.1 & 9.5 & 9.2 & 9.1 & 8.6 & 8.5 & 8.2 & 8.4 & 8.1 & 7.9 & 8.2 & 8.6 & 8.6 & 8.6 & 8.6 & 8.8 & 8.7 & 8.9 & 8.6 & 7.9 & 7.5 & 6.9 & 7.2 & 7.4 & 7.3 & 7.2 & 6.8 & 6.5 & 6.5 & 6.5 & 6.5 & 6.5 & 6.6 \\
\hline vico & $\mathrm{m}$ & $\mathrm{m}$ & m & m & $\mathrm{m}$ & 0.2 & 0.2 & 0.3 & 0.3 & 0.5 & 0.5 & 0.5 & 0.6 & 0.6 & 0.7 & 0.7 & 0.7 & 0.7 & 0.8 & 0.9 & 0.9 & 0.9 & 1.0 & 1.1 & 1.1 & 1.2 & 1.2 & 1.4 & 1.8 & 1.6 & 1.7 & 1.4 & $m$ \\
\hline therland & 6.4 & 6.3 & 6.5 & 6.4 & 6.1 & 6.2 & 6.1 & 6.2 & 6.1 & 6.0 & 6.7 & 6.6 & 6.6 & 6.5 & 5.9 & 5.8 & 5.7 & 5.5 & 5.4 & 5.2 & 5.0 & 4.9 & 5.0 & 5.1 & 5.0 & 5.0 & 4.8 & 4.7 & 4.7 & 4.7 & 4.6 & 4.7 & 4.8 \\
\hline w Zeal & 7.1 & 7.1 & 7.9 & 7.4 & 7.2 & 7.6 & 6.9 & 6.7 & 6.7 & 7.0 & 7.4 & 7.8 & 7.1 & 6.5 & 6.0 & 5.7 & 5.5 & 5.4 & 5.3 & 5.1 & 5.0 & 4.8 & 4.6 & 4.5 & 4.3 & 4.3 & 4.3 & 4.3 & 4.5 & 4.8 & 5.0 & 5.1 & 5.1 \\
\hline Norway & 4.5 & $\mathrm{~m}$ & $\mathrm{~m}$ & $\mathrm{~m}$ & $\mathrm{~m}$ & 4.7 & $\mathrm{~m}$ & $\mathrm{~m}$ & 5.5 & 5.6 & 5.6 & 5.7 & 5.7 & 5.7 & 5.6 & 5.5 & 5.3 & 5.1 & 5.5 & 5.4 & 4.8 & 4.9 & 5.2 & 5.3 & 5.1 & 4.8 & 4.6 & 4.7 & 4.8 & 4.9 & 5.1 & 5.2 & 5.3 \\
\hline bland & $\mathrm{m}$ & $\mathrm{m}$ & $\mathrm{m}$ & $\mathrm{m}$ & $\mathrm{m}$ & $\mathrm{m}$ & $\mathrm{m}$ & $\mathrm{m}$ & $\mathrm{m}$ & $\mathrm{m}$ & 5.1 & 7.9 & 9.4 & 9.7 & 9.6 & 9.4 & 9.4 & 9.7 & 9.2 & 9.4 & 10.5 & 11.5 & 11.7 & 11.9 & 11.7 & 11.4 & 11.5 & 10.6 & 10.4 & 10.2 & 9.9 & 9.7 & 9.5 \\
\hline Portugal & 3.7 & 4.0 & 3.8 & 4.3 & 4.1 & 4.1 & 4.3 & 4.5 & 4.4 & 4.4 & 4.9 & 5.3 & 5.8 & 6.4 & 6.5 & 7.2 & 7.4 & 7.3 & 7.4 & 7.7 & 7.9 & 8.3 & 8.8 & 9.3 & 9.9 & 10.3 & 10.6 & 10.8 & 10.9 & 11.1 & 11.2 & 11.3 & 11.3 \\
\hline Slovak $R$ & $\mathrm{~m}$ & $\mathrm{~m}$ & $\mathrm{~m}$ & $\mathrm{~m}$ & $\mathrm{~m}$ & $\mathrm{~m}$ & $\mathrm{~m}$ & $\mathrm{~m}$ & $\mathrm{~m}$ & $\mathrm{~m}$ & $\mathrm{~m}$ & $\mathrm{~m}$ & $\mathrm{~m}$ & $\mathrm{~m}$ & $\mathrm{~m}$ & 6.3 & 6.3 & 6.3 & 6.4 & 6.4 & 6.3 & 6.4 & 6.4 & 6.2 & 6.2 & 6.2 & 6.0 & 5.8 & 5.8 & 5.7 & 5.7 & 5.6 & 5.6 \\
\hline Slovenia & $\mathrm{m}$ & $\mathrm{m}$ & $\mathrm{m}$ & $\mathrm{m}$ & $\mathrm{m}$ & $\mathrm{m}$ & $\mathrm{m}$ & $\mathrm{m}$ & $\mathrm{m}$ & $\mathrm{m}$ & $\mathrm{m}$ & $\mathrm{m}$ & $\mathrm{m}$ & $\mathrm{m}$ & $\mathrm{m}$ & $\mathrm{m}$ & 10.5 & 10.5 & 10.6 & 10.5 & 10.6 & 10.8 & 11.0 & 10.3 & 10.2 & 9.9 & 10.0 & 9.6 & 9.7 & 9.8 & 9.8 & 9.9 & 10.0 \\
\hline ain & 6.2 & 6.8 & 6.9 & 7.2 & 7.4 & 7.5 & 7.5 & 7.4 & 7.3 & 7.4 & 7.9 & 8.1 & 8.4 & 8.9 & 8.9 & 9.0 & 9.2 & 9.1 & 8.8 & 8.6 & 8.6 & 8.3 & 8.3 & 8.2 & 8.1 & 8.1 & 8.0 & 8.0 & 8.2 & 8.4 & 8.5 & 8.6 & 8.6 \\
\hline eden & 7.1 & 7.7 & 7.7 & 7.8 & 7.5 & 7.6 & 7.7 & 7.6 & 7.7 & 7.7 & 7.7 & 8.0 & 8.6 & 8.7 & 8.5 & 8.2 & 8.2 & 7.9 & 7.7 & 7.5 & 7.2 & 7.2 & 7.2 & 7.8 & 7.7 & 7.6 & 7.3 & 7.2 & 7.2 & 7.2 & 7.3 & 7.2 & 7.2 \\
\hline witzerla & 5. & 5.6 & 6.1 & 6.0 & 6.3 & 6.1 & 6.1 & 6.1 & 6.1 & 5.8 & 5.6 & 5.9 & 6.2 & 6.5 & 6.5 & 6.7 & 6.7 & 6.8 & 6.8 & 6.8 & 6.6 & 6.8 & 6.8 & 6.9 & 6.8 & 6.8 & 6.5 & 6.4 & 6.4 & 6.4 & 6.3 & 6.4 & 6.4 \\
\hline Turkey & 1.2 & 1.2 & 1.4 & 1.5 & 1.4 & 1.3 & 1.3 & 1.3 & 1.5 & 1.8 & 2.4 & 2.4 & 2.6 & 2.7 & 2.7 & 2.7 & 3.2 & 3.9 & 3.9 & 4.7 & 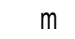 & m & $\mathrm{m}$ & $\mathrm{m}$ & $\mathrm{m}$ & 5.9 & 5.8 & 6.1 & $m$ & $m$ & $m$ & $m$ & $m$ \\
\hline ited Ki & 5.5 & 5.8 & 5.9 & 5.8 & 5.7 & 5.6 & 5.5 & 5.3 & 4.9 & 4.7 & 4.8 & 5.2 & 5.5 & 5.6 & 5.4 & 5.4 & 5.4 & 5.3 & 5.2 & 5.3 & 5.3 & 5.5 & 5.4 & 5.4 & 5.5 & 5.6 & 5.3 & 5.4 & 5.4 & 5.4 & 5.4 & 5.5 & 5.5 \\
\hline United States & 6.2 & 6.4 & 6.8 & 6.7 & 6.4 & 6.2 & 6.2 & 6.1 & 6.1 & 6.0 & 6.1 & 6.3 & 6.3 & 6.3 & 6.3 & 6.3 & 6.2 & 6.1 & 6.0 & 5.9 & 5.9 & 5.9 & 6.1 & 6.1 & 6.0 & 5.9 & 5.9 & 6.0 & 6.1 & 6.8 & 6.9 & 6.7 & 6.7 \\
\hline
\end{tabular}


DELSA/ELSA/WD/SEM(2011)9

Table A.I.1.4b: Public social expenditures on income support to the working-age population as \% GDP, 1980 - 2012, estimated for 2008 to 2012

\begin{tabular}{|c|c|c|c|c|c|c|c|c|c|c|c|c|c|c|c|c|c|c|c|c|c|c|c|c|c|c|c|c|c|c|c|c|c|}
\hline & 1980 & 1981 & 1982 & 1983 & 1984 & 1985 & 1986 & 1987 & 1988 & 1989 & 1990 & 1991 & 1992 & 1993 & 1994 & 1995 & 1996 & 1997 & 1998 & 1999 & 2000 & 2001 & 2002 & 2003 & 2004 & 2005 & 2006 & 2007 & 2008 & 2009 & 2010 & 2011 & 2012 \\
\hline Istralia & 2.5 & 2.5 & 3.2 & 3.5 & 3.4 & 3.3 & 3.2 & 3.0 & 2.9 & 3.1 & 4.0 & 4.6 & 5.1 & 5.4 & 5.2 & 5.3 & 5.5 & 5.4 & 5.3 & 5.1 & 5.3 & 5.2 & 4.9 & 5.2 & 4.8 & 4.5 & 4.2 & \begin{tabular}{ll|}
4.0 \\
\end{tabular} & 4.3 & 4.8 & 4.1 & 4.0 & 3.8 \\
\hline tria & 6.0 & $\mathrm{~m}$ & $\mathrm{~m}$ & $\mathrm{~m}$ & $\mathrm{~m}$ & 6.2 & $\mathrm{~m}$ & $\mathrm{~m}$ & $\mathrm{~m}$ & $\mathrm{~m}$ & 5.7 & 5.8 & 6.2 & 6.7 & 7.0 & 6.8 & 6.7 & 6.2 & 6.0 & 6.0 & 6.1 & 6.0 & 6.2 & 6.4 & 6.2 & 6.0 & 5.7 & 5.3 & 5.3 & 5.8 & 5.8 & 5.8 & 5.8 \\
\hline elgium & 9.1 & 9.9 & 10.0 & 10.2 & 9.8 & 9.6 & 9.3 & 8.9 & 8.1 & 7.7 & 7.9 & 8.2 & 8.0 & 8.3 & 8.0 & 8.0 & 8.0 & 7.8 & 7.3 & 7.1 & 6.7 & 6.8 & 7.0 & 7.3 & 7.2 & 7.2 & 7.1 & 7.2 & 7.4 & 8.1 & 8.0 & 7.8 & 7.7 \\
\hline anada & 2.7 & 2.8 & 3.9 & 3.8 & 3.6 & 3.5 & 3.5 & 3.3 & 3.2 & 3.2 & 3.6 & 4.5 & 4.4 & 4.5 & 4.0 & 3.6 & 3.4 & 3.1 & 3.0 & 2.8 & 2.7 & 2.9 & 2.9 & 2.9 & 2.8 & 2.7 & 2.6 & 2.5 & 2.6 & 2.8 & 2.8 & 2.8 & 2.7 \\
\hline Chile & $\mathrm{m}$ & $\mathrm{m}$ & $\mathrm{m}$ & $\mathrm{m}$ & $\mathrm{m}$ & $\mathrm{m}$ & $\mathrm{m}$ & 1.6 & 1.4 & 1.2 & 1.3 & 1.3 & 1.2 & 1.2 & 1.2 & 1.2 & 1.3 & 1.2 & 1.3 & 1.3 & 1.3 & 1.3 & 1.3 & 1.2 & 1.1 & 1.0 & 0.9 & 0.9 & 0.9 & 1.2 & 1.0 & $m$ & m \\
\hline $\operatorname{ech} R$ & $\mathrm{~m}$ & $\mathrm{~m}$ & $\mathrm{~m}$ & $\mathrm{~m}$ & $\mathrm{~m}$ & $\mathrm{~m}$ & $\mathrm{~m}$ & $\mathrm{~m}$ & $\mathrm{~m}$ & $\mathrm{~m}$ & 4.8 & 5.1 & 5.0 & 4.4 & 4.5 & 4.6 & 4.5 & 4.5 & 4.2 & 4.4 & 4.6 & 4.5 & 4.7 & 4.8 & 4.4 & 4.1 & 4.1 & 4.4 & 4.4 & 4.7 & 4.7 & 4.8 & 4.7 \\
\hline enmark & 9.7 & 9.4 & 9.4 & 10.0 & 9.3 & 8.8 & 8.3 & 8.6 & 9.4 & 9.5 & 9.1 & 9.5 & 9.7 & 10.5 & 10.6 & 10.1 & 9.6 & 9.1 & 8.5 & 8.0 & 7.8 & 8.0 & 8.2 & 8.8 & 8.8 & 8.2 & 7.4 & 7.0 & 7.0 & 7.9 & 8.0 & 8.1 & 8.1 \\
\hline onia & $\mathrm{m}$ & $\mathrm{m}$ & $\mathrm{m}$ & $\mathrm{m}$ & $\mathrm{m}$ & $\mathrm{m}$ & $\mathrm{m}$ & $\mathrm{m}$ & $\mathrm{m}$ & $\mathrm{m}$ & $\mathrm{m}$ & $\mathrm{m}$ & $\mathrm{m}$ & $\mathrm{m}$ & $\mathrm{m}$ & $\mathrm{m}$ & $\mathrm{m}$ & $\mathrm{m}$ & 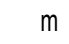 & m. & 3.3 & 3.3 & 3.2 & 3.1 & 3.5 & 3.3 & 3.1 & 3.1 & 3.9 & 5.4 & 5.7 & 5.2 & 4.9 \\
\hline ance & 4.5 & 4.8 & 4.9 & 4.9 & 4.7 & 7.0 & 6.7 & 6.5 & 6.2 & 5.9 & 5.0 & 5.1 & 5.1 & 5.3 & 5.2 & 5.1 & 5.2 & 5.2 & 5.1 & 5.1 & 4.8 & 4.8 & 5.1 & 5.3 & 5.3 & 5.1 & 4.8 & 4.6 & 4.6 & 5.0 & 5.1 & 5.0 & 4.9 \\
\hline ermany & 4.4 & 4.7 & 4.7 & 4.6 & 4.3 & 4.2 & 4.1 & 4.2 & 4.2 & 4.0 & 3.8 & 4.7 & 4.7 & 5.2 & 5.1 & 4.9 & 4.9 & 4.8 & 4.6 & 4.5 & 4.4 & 4.5 & 4.6 & 4.8 & 4.8 & 4.8 & 4.3 & 4.0 & 3.9 & 4.3 & 4.1 & 4.0 & 3.9 \\
\hline reece & 1.5 & 1.9 & 2.2 & 2.3 & 2.2 & 2.4 & 2.4 & 2.4 & 2.2 & 2.3 & 2.0 & 2.0 & 1.8 & 1.8 & 2.0 & 2.0 & 1.9 & 1.9 & 2.0 & 1.9 & 1.9 & 1.9 & 1.9 & 2.0 & 2.0 & 2.0 & 1.9 & 2.0 & 2.2 & 2.4 & 2.3 & 2.4 & 2.4 \\
\hline ngary & $\mathrm{m}$ & $\mathrm{m}$ & $\mathrm{m}$ & $\mathrm{m}$ & $\mathrm{m}$ & $\mathrm{m}$ & $\mathrm{m}$ & $\mathrm{m}$ & $\mathrm{m}$ & $\mathrm{m}$ & $\mathrm{m}$ & 0.0 & 2.2 & 2.1 & 1.2 & 0.9 & 0.7 & 0.6 & 0.6 & 5.3 & 5.0 & 4.8 & 4.9 & 5.2 & 5.0 & 5.1 & 5.3 & 5.3 & 5.5 & 5.7 & 5.4 & 5.5 & 5.1 \\
\hline Iceland & $\pi$ & $\mathrm{m}$ & $\mathrm{m}$ & $\mathrm{m}$ & $\mathrm{m}$ & $\mathrm{m}$ & $\mathrm{m}$ & $\mathrm{m}$ & $\mathrm{m}$ & $\mathrm{m}$ & 3.2 & 3.3 & 3.5 & 3.8 & 3.7 & 3.9 & 3.7 & 3.5 & 3.1 & 3.0 & 3.0 & 3.0 & 3.5 & 4.1 & 4.1 & 3.7 & 3.4 & 3.7 & 3.9 & 5.2 & 5.0 & 4.7 & 4.4 \\
\hline and & 3.5 & 3.6 & 4.1 & 4.1 & 4.1 & 7.5 & 7.7 & 7.4 & 6.7 & 6.1 & 4.8 & 5.2 & 5.5 & 5.5 & 5.2 & 5.2 & 4.8 & 4.2 & 3.8 & 4.3 & 3.9 & 4.1 & 4.7 & 4.9 & 5.0 & 4.9 & 5.0 & 5.3 & 6.3 & 7.8 & 8.1 & 7.5 & 6.9 \\
\hline Israel & $\mathrm{m}$ & $\mathrm{m}$ & $\mathrm{m}$ & $\mathrm{m}$ & $\mathrm{m}$ & $\mathrm{m}$ & $\mathrm{m}$ & $\mathrm{m}$ & $\mathrm{m}$ & $\mathrm{m}$ & $\mathrm{m}$ & $\mathrm{m}$ & $\mathrm{m}$ & $\mathrm{m}$ & $\mathrm{m}$ & 4.6 & 4.8 & 5.0 & 5.1 & 5.2 & 5.2 & 5.7 & 5.6 & 5.2 & 4.5 & 4.3 & 4.2 & 4.0 & 4.1 & 4.3 & 4.3 & 4.3 & 4.2 \\
\hline Italy & 3.3 & 4.0 & 4.0 & 4.2 & 4.1 & 4.0 & 3.9 & 3.7 & 3.6 & 3.7 & 3.1 & 2.9 & 3.1 & 3.2 & 3.1 & 2.8 & 2.8 & 2.8 & 2.6 & 2.6 & 2.5 & 2.4 & 2.6 & 2.7 & 2.8 & 2.7 & 2.8 & 2.8 & 2.9 & 3.1 & 3.1 & 3.1 & 3.0 \\
\hline Japan & 1. & 1.6 & 1.6 & 1.6 & 1.5 & 1.4 & 1.4 & 1.4 & 1.3 & 1.2 & 1.1 & 1.1 & 1.2 & 1.3 & 1.4 & 1.5 & 1.4 & 1.5 & 1.6 & 1.6 & 1.6 & 1.7 & 1.7 & 1.6 & 1.5 & 1.5 & 1.6 & 1.6 & 1.7 & $m$ & $m$ & $m$ & $m$ \\
\hline Luxembo & 6.1 & 6.4 & 6.2 & 6.4 & 6.2 & 6.2 & 5.9 & 6.0 & 5.6 & 5.4 & 5.0 & 5.2 & 5.2 & 5.5 & 5.5 & 5.8 & 5.8 & 5.9 & 6.0 & 5.9 & 5.7 & 6.5 & 6.8 & 7.2 & 7.0 & 6.6 & 6.2 & 5.7 & 6.0 & 6.9 & 6.6 & 6.5 & 6.5 \\
\hline xico & $\mathrm{m}$ & $\mathrm{m}$ & $\mathrm{m}$ & $\mathrm{m}$ & $\mathrm{m}$ & 0.0 & 0.0 & 0.0 & 0.0 & 0.0 & 0.1 & 0.1 & 0.2 & 0.2 & 0.5 & 0.5 & 0.4 & 0.3 & 0.4 & 0.4 & 0.4 & 1.0 & 1.0 & 1.0 & 1.0 & 1.0 & 1.0 & 0.9 & 1.1 & 1.2 & 1.2 & 1.2 & $m$ \\
\hline therlano & 11.4 & 11.9 & 13.0 & 13.4 & 12.8 & 11.5 & 11.0 & 10.7 & 10.5 & 10.0 & 10.8 & 10.7 & 10.7 & 10.7 & 9.6 & 9.2 & 8.6 & 7.8 & 7.1 & 6.6 & 6.2 & 6.1 & 6.2 & 6.5 & 6.5 & 6.0 & 5.7 & 5.3 & 5.4 & 6.0 & 6.1 & 6.0 & 5.9 \\
\hline ew Zeal & 4.1 & 3.9 & 4.1 & 4.4 & 4.3 & 4.7 & 5.3 & 5.8 & 6.5 & 7.4 & 7.3 & 6.9 & 7.1 & 6.6 & 6.1 & 5.9 & 6.0 & 6.8 & 6.8 & 6.5 & 6.2 & 5.9 & 5.8 & 5.6 & 5.2 & 5.2 & 5.6 & 5.1 & 5.7 & 5.9 & 5.8 & 5.7 & 5.3 \\
\hline Norway & 5.0 & 0.0 & 0.0 & 0.0 & 0.0 & 5.7 & 0.4 & 0.4 & 6.8 & 7.4 & 7.5 & 7.8 & 8.2 & 8.0 & 7.7 & 7.4 & 7.1 & 6.8 & 7.0 & 7.0 & 6.4 & 6.6 & 7.1 & 7.5 & 6.7 & 6.0 & 5.5 & 5.4 & 5.2 & 6.0 & 6.1 & 5.8 & 5.7 \\
\hline land & $\mathrm{m}$ & $\mathrm{m}$ & $\mathrm{m}$ & $\mathrm{m}$ & $\mathrm{m}$ & $\mathrm{m}$ & $\mathrm{m}$ & $\mathrm{m}$ & $\mathrm{m}$ & $\mathrm{m}$ & 4.8 & 7.9 & 9.7 & 9.0 & 8.6 & 8.1 & 7.9 & 7.3 & 6.9 & 6.9 & 5.3 & 5.4 & 5.3 & 5.0 & 4.5 & 4.3 & 3.9 & 3.5 & 3.5 & 3.7 & 3.7 & 3.7 & 3.6 \\
\hline ortugal & 2.9 & 3.1 & 3.2 & 3.1 & 3.1 & 2.9 & 3.0 & 3.1 & 3.0 & 2.9 & 3.2 & 3.5 & 3.7 & 4.1 & 4.2 & 3.8 & 3.8 & 3.8 & 3.8 & 3.6 & 3.7 & 3.6 & 3.8 & 4.1 & 4.2 & 4.2 & 4.2 & 4.0 & 4.2 & 4.7 & 4.7 & 4.8 & 4.8 \\
\hline ovak Re & $\mathrm{m}$ & $\mathrm{m}$ & $\mathrm{m}$ & $\mathrm{m}$ & $\mathrm{m}$ & $\mathrm{m}$ & $\mathrm{m}$ & $\mathrm{m}$ & $\mathrm{m}$ & $\mathrm{m}$ & $\mathrm{m}$ & $\mathrm{m}$ & $\mathrm{m}$ & $\mathrm{m}$ & $\mathrm{m}$ & 5.5 & 5.3 & 5.3 & 5.6 & 5.9 & 5.4 & 4.9 & 4.8 & 4.3 & 3.7 & 3.6 & 3.7 & 3.5 & 3.5 & 4.2 & 4.2 & 4.1 & 4.0 \\
\hline Slovenia & $\mathrm{m}$ & $\mathrm{m}$ & $\mathrm{m}$ & $\mathrm{m}$ & $\mathrm{m}$ & $\mathrm{m}$ & $\mathrm{m}$ & $\mathrm{m}$ & $\mathrm{m}$ & $\mathrm{m}$ & $\mathrm{m}$ & $\mathrm{m}$ & $\mathrm{m}$ & $\mathrm{m}$ & $\mathrm{m}$ & $\mathrm{m}$ & 5.0 & 5.3 & 5.2 & 5.3 & 5.1 & 4.9 & 4.7 & 4.7 & 4.6 & 4.7 & 4.4 & 3.9 & 4.0 & 4.5 & 4.6 & 4.7 & 4.6 \\
\hline Spain & 4.8 & 5.2 & 5.0 & 5.0 & 4.9 & 5.4 & 5.0 & 4.9 & 4.8 & 4.7 & 5.6 & 6.3 & 6.8 & 7.6 & 6.7 & 5.9 & 5.5 & 5.1 & 4.8 & 4.6 & 4.6 & 4.6 & 4.8 & 4.9 & 5.0 & 5.1 & 5.0 & 5.1 & 5.4 & 6.3 & 6.6 & 6.6 & 6.4 \\
\hline den & 6.8 & 7.0 & 7.0 & 7.1 & 6.8 & 7.3 & 7.5 & 7.7 & 8.3 & 8.0 & 8.4 & 9.1 & 9.6 & 9.9 & 9.3 & 8.5 & 7.8 & 7.4 & 7.3 & 7.1 & 6.9 & 6.9 & 7.0 & 7.2 & 7.0 & 6.7 & 6.3 & 5.6 & 5.5 & 6.1 & 5.7 & 5.5 & 5.3 \\
\hline vitzerla & 3 & 3.7 & 3.9 & 4.2 & 4.1 & 4.0 & 4.0 & 4.0 & 4.0 & 3.8 & 2.8 & 3.2 & 3.9 & 4.7 & 4.6 & 4.5 & 4.7 & 4.9 & 4.8 & 4.5 & 4.2 & 4.1 & 4.5 & 5.0 & 5.0 & 5.0 & 4.7 & 4.3 & 4.2 & 4.6 & 4.8 & 4.7 & 4.5 \\
\hline Turkey & 1. & 1.0 & 1.0 & 1.1 & 1.0 & 0.9 & 1.0 & 1.1 & 1.2 & 1.4 & 1.6 & 1.8 & 1.7 & 1.5 & 1.2 & 1.0 & 1.8 & 1.7 & 1.6 & 1.9 & m & III & 0.0 & 0.0 & 0.0 & 0.2 & 0.1 & 0.1 & $m$ & $m$ & $m$ & $m$ & III \\
\hline United K & 4.5 & 5.2 & 5.7 & 5.9 & 6.1 & 6.1 & 6.1 & 5.7 & 5.3 & 5.0 & 4.2 & 4.8 & 5.4 & 5.7 & 5.4 & 5.3 & 5.1 & 4.7 & 4.4 & 4.3 & 4.3 & 4.6 & 4.3 & 4.5 & 4.6 & 4.5 & 4.5 & 4.5 & 4.8 & 5.5 & 5.5 & 5.4 & 5.3 \\
\hline United States & 2.3 & 2.3 & 2.2 & 2.4 & 1.9 & 1.8 & 1.8 & 1.7 & 1.7 & 1.6 & 1.7 & 2.0 & 2.2 & 2.2 & 2.1 & 2.0 & 2.0 & 1.8 & 1.8 & 1.7 & 1.7 & 2.1 & 2.2 & 2.1 & 2.0 & 1.9 & 1.9 & 2.0 & 2.1 & 2.8 & 3.1 & 2.9 & 2.6 \\
\hline
\end{tabular}

Note: Social spending aggregates based on detailed data for 1980-2007; aggregate spending estimates for 2008-2012. 


\section{DELSA/ELSA/WD/SEM(2011)9}

\section{Table A.l.1.4c: Public social expenditures on Health as \% GDP, 1980 - 2012, estimated for 2008 to 2012}

\begin{tabular}{|c|c|c|c|c|c|c|c|c|c|c|c|c|c|c|c|c|c|c|c|c|c|c|c|c|c|c|c|c|c|c|c|c|c|}
\hline & 980 & 981 & 982 & 983 & & 1985 & 1986 & $198 \%$ & 808 & & 190 & g91 & 992 & & 994 & 1995 & 1996 & Ty9t & 1998 & & & & & & & & 2006 & 2000 & 2008 & 200 & & & \\
\hline Australia & 3.8 & 3.7 & 3.7 & 3.9 & 4.4 & 4.5 & 4.6 & 4.4 & 4.3 & 4.3 & 4.4 & 4.6 & 4.6 & 4.6 & 4.7 & 4.7 & 4.8 & 5.0 & 5.1 & 5.3 & 5.4 & 5.4 & 5.6 & 5.5 & 5.7 & 5.6 & 5.6 & \begin{tabular}{|l|}
5.7 \\
\end{tabular} & 5.7 & 6.1 & 6.1 & 6.1 & $\overline{6.2}$ \\
\hline ustria & 5.1 & 5.0 & 4.9 & 4.8 & 4.8 & 4.9 & 5.1 & 5.2 & 5.2 & 5.2 & 5.4 & 5.4 & 5.7 & 6.0 & 6.1 & 5.9 & 5.9 & 6.4 & 6.5 & 6.7 & 6.6 & 6.6 & 6.6 & 6.7 & 6.8 & 6.9 & 6.7 & 6.8 & 6.8 & 6.9 & 6.9 & 6.9 & 7.0 \\
\hline Igium & 2 & 5.5 & 5.9 & 5.8 & 5.7 & 5.7 & 5.7 & 6.0 & 6.5 & 6.4 & 6.4 & 6.7 & 6.9 & 7.0 & 6.8 & 6.5 & 6.7 & 6.3 & 6.4 & 6.5 & 6.6 & 6.7 & 6.7 & 7.1 & 7.5 & 7.4 & 7.2 & 7.3 & 7.4 & 7.4 & 7.4 & 7.5 & 7.5 \\
\hline nada & 5.1 & 5.4 & 6.0 & 6.1 & 6.0 & 6.1 & 6.1 & 6.1 & 6.1 & 6.3 & 6.6 & 7.1 & 7.2 & 7.0 & 6.7 & 6.4 & 6.2 & 6.1 & 6.3 & 6.1 & 6.2 & 6.5 & 6.6 & 6.8 & 6.8 & 6.8 & 6.9 & 7.0 & 7.7 & 8.5 & 8.4 & 8.5 & 8.7 \\
\hline ille & $\mathrm{m}$ & $\mathrm{m}$ & $\mathrm{m}$ & $\mathrm{m}$ & $\mathrm{m}$ & $\mathrm{m}$ & $\mathrm{m}$ & $\mathrm{m}$ & $\mathrm{m}$ & $\mathrm{m}$ & $\mathrm{m}$ & $\mathrm{m}$ & $\mathrm{m}$ & $\mathrm{m}$ & $\mathrm{m}$ & 2.6 & 2.7 & 2.8 & 3.1 & 3.3 & 3.4 & 3.6 & 3.7 & 3.7 & 3.5 & 3.5 & 3.4 & 3.7 & 3.8 & 4.5 & 4.3 & m & $m$ \\
\hline ech & $\mathrm{m}$ & $\mathrm{m}$ & $\mathrm{m}$ & $\mathrm{m}$ & $\mathrm{m}$ & $\mathrm{m}$ & $\mathrm{m}$ & $\mathrm{m}$ & $\mathrm{m}$ & $\mathrm{m}$ & 4.6 & 4.8 & 4.9 & 6.4 & 6.5 & 6.4 & 6.1 & 6.0 & 6.0 & 5.9 & 5.9 & 6.0 & 6.4 & 6.7 & 6.4 & 6.3 & 6.0 & 5.8 & 5.8 & 5.9 & 5.9 & 6.0 & 6.1 \\
\hline nmark & 5.5 & 5.6 & 5.6 & 5.3 & 5.1 & 5.1 & 4.8 & 5.0 & 5.1 & 5.0 & 4.7 & 4.7 & 4.7 & 5.0 & 4.8 & 4.6 & 4.6 & 4.6 & 5.0 & 5.2 & 5.1 & 5.3 & 5.5 & 6.0 & 6.1 & 6.1 & 6.2 & 6.5 & 6.5 & 6.6 & 6.6 & 6.7 & 6.7 \\
\hline tonia & $\mathrm{m}$ & $\mathrm{m}$ & $\mathrm{m}$ & $\mathrm{m}$ & $\mathrm{m}$ & $\mathrm{m}$ & $\mathrm{m}$ & $\mathrm{m}$ & $\mathrm{m}$ & $\mathrm{m}$ & $\mathrm{m}$ & $\mathrm{m}$ & $\mathrm{m}$ & $\mathrm{m}$ & $\mathrm{m}$ & $\mathrm{m}$ & $\mathrm{m}$ & $\mathrm{m}$ & $\mathrm{m}$ & $\mathrm{m}$ & 4.1 & 3.8 & 3.7 & 3.8 & 3.9 & 3.9 & 3.7 & 4.0 & 4.0 & 4.1 & 4.1 & 4.1 & 4.2 \\
\hline land & 5.0 & 5.2 & 5.3 & 5.3 & 5.3 & 5.6 & 5.7 & 5.8 & 5.7 & 5.7 & 6.3 & 7.2 & 7.2 & 6.3 & 5.8 & 5.7 & 5.8 & 5.5 & 5.3 & 5.3 & 5.1 & 5.3 & 5.6 & 5.9 & 6.0 & 6.2 & 6.3 & 6.1 & 6.1 & 6.2 & 6.2 & 6.3 & 6.3 \\
\hline ance & 5.6 & 5.9 & 5.9 & 6.0 & 6.1 & 6.3 & 6.4 & 6.6 & 6.6 & 6.5 & 6.2 & 6.3 & 6.5 & 6.9 & 6.8 & 7.4 & 7.4 & 7.3 & 7.2 & 7.2 & 7.1 & 7.2 & 7.4 & 7.7 & 7.7 & 7.7 & 7.6 & 7.5 & 7.5 & 7.5 & 7.6 & 7.6 & 7.7 \\
\hline any & 6.6 & 6.8 & 6.7 & 6.6 & 6.7 & 6.8 & 6.7 & 6.8 & 6.9 & 6.3 & 6.3 & 6.9 & 7.8 & 7.7 & 7.9 & 8.2 & 8.4 & 8.1 & 8.1 & 8.1 & 8.1 & 8.1 & 8.3 & 8.4 & 8.0 & 8.1 & 7.9 & 7.8 & 7.9 & 8.0 & 8.1 & 8.1 & 3.2 \\
\hline eece & 3.3 & 4.5 & 4.7 & 4.9 & 4.8 & 4.5 & 4.3 & 4.0 & 3.2 & 3.6 & 3.5 & 3.4 & 3.8 & 4.3 & 4.3 & 4.5 & 4.5 & 4.5 & 4.4 & 4.6 & 4.7 & 5.3 & 5.3 & 5.3 & 5.1 & 5.8 & 6.0 & 5.9 & 5.9 & 5.9 & 6.0 & 6.0 & 6.1 \\
\hline ngary & $\mathrm{m}$ & $\mathrm{m}$ & $\mathrm{m}$ & $\mathrm{m}$ & $\mathrm{m}$ & $\mathrm{m}$ & $\mathrm{m}$ & $\mathrm{m}$ & $\mathrm{m}$ & $\mathrm{m}$ & $\mathrm{m}$ & 6.3 & 6.7 & 6.6 & 7.1 & 6.1 & 5.7 & 5.5 & 5.3 & 5.2 & 5.0 & 4.9 & 5.3 & 6.1 & 5.8 & 6.0 & 5.9 & 5.2 & 5.2 & 5.2 & 5.2 & 5.3 & 5.3 \\
\hline nd & $\mathrm{m}$ & $\mathrm{m}$ & $\mathrm{m}$ & $\mathrm{m}$ & $\mathrm{m}$ & $\mathrm{m}$ & $\mathrm{m}$ & $\mathrm{m}$ & $\mathrm{m}$ & $\mathrm{m}$ & 5.8 & 6.0 & 5.9 & 5.9 & 5.8 & 5.9 & 5.7 & 5.6 & 6.1 & 6.8 & 6.5 & 6.4 & 6.8 & 6.6 & 6.2 & 5.8 & 5.6 & 5.7 & 5.9 & 6.3 & 5.2 & 5.0 & 4.1 \\
\hline land & 6.8 & 6.5 & 6.2 & 6.1 & 5.8 & 5.7 & 5.6 & 5.2 & 4.8 & 4.5 & 4.4 & 4.7 & 5.0 & 5.0 & 5.0 & 4.8 & 4.7 & 4.7 & 4.6 & 4.6 & 4.6 & 5.1 & 5.4 & 5.6 & 5.9 & 5.8 & 5.7 & 5.8 & 5.8 & 5.9 & 5.9 & 5.9 & 5.9 \\
\hline Israel & $\mathrm{m}$ & $\mathrm{m}$ & $\mathrm{m}$ & $\mathrm{m}$ & m & $\mathrm{m}$ & $\mathrm{m}$ & $\mathrm{m}$ & $\mathrm{m}$ & $\mathrm{m}$ & $\mathrm{m}$ & $\mathrm{m}$ & $\mathrm{m}$ & $\mathrm{m}$ & $\mathrm{m}$ & 5.1 & 5.3 & 5.2 & 5.0 & 4.8 & 4.7 & 4.9 & 5.0 & 4.9 & 4.7 & 4.6 & 4.4 & 4.3 & 4.4 & 4.3 & 4.2 & 4.1 & 4.0 \\
\hline Italy & 5.5 & 5.3 & 5.4 & 5.4 & 5.3 & 5.3 & 5.2 & 5.6 & 5.7 & 5.7 & 6.1 & 6.3 & 6.2 & 5.9 & 5.6 & 5.1 & 5.2 & 5.4 & 5.4 & 5.5 & 5.8 & 6.1 & 6.2 & .2 & 6.6 & 6.8 & 6.9 & 3.6 & 6.6 & 6.6 & 6.6 & 6.7 & 6.7 \\
\hline pan & 4.5 & 4.6 & 4.7 & 4.9 & 4.7 & 4.6 & 4.7 & 4.8 & 4.7 & 4.6 & 4.5 & 4.6 & 4.8 & 5.1 & 5.3 & 5.7 & 5.8 & 5.7 & 5.9 & 6.1 & 5.9 & 6.2 & 6.1 & 6.1 & 6.1 & 6.3 & 6.2 & 6.3 & 6.7 & $m$ & $m$ & $m$ & $m$ \\
\hline rea & $\mathrm{m}$ & $\mathrm{m}$ & $\mathrm{m}$ & $\mathrm{m}$ & $\mathrm{m}$ & $\mathrm{m}$ & $\mathrm{m}$ & $\mathrm{m}$ & $\mathrm{m}$ & $\mathrm{m}$ & 1.5 & 1.4 & 1.4 & 1.4 & 1.4 & 1.4 & 1.6 & 1.7 & 2.0 & 2.1 & 2.2 & 2.8 & 2.6 & 2.7 & 2.8 & 3.0 & 3.3 & 3.5 & 3.6 & 3.8 & 3.8 & 4.1 & 4.3 \\
\hline herlan & 5. & 5.2 & 5.5 & 5.4 & 5.2 & 5.2 & 5.1 & 5.2 & 5.1 & 5.3 & 5.4 & 5.6 & 6.1 & 6.2 & 6.1 & 5.9 & 5.4 & 5.4 & 5.2 & 5.1 & 5.0 & 5.2 & 5.5 & 5.8 & 5.8 & 9 & 5.9 & 6.0 & 6.0 & 6.0 & 6.1 & 6.1 & 6.2 \\
\hline w Zea & 5. & 5.5 & 5.2 & 5.1 & 4.8 & 4.4 & 4.4 & 5.0 & 5.4 & 5.5 & 5.6 & 6.0 & 5.8 & 5.4 & 5.5 & 5.5 & 5.4 & 5.6 & 5.9 & 5.9 & 6.0 & 5.9 & 6.3 & 6.2 & 6. & 6.7 & 7.1 & 7.1 & 7.8 & 8.5 & 8.8 & 9.2 & 9.4 \\
\hline iway & 4.9 & $\mathrm{~m}$ & $\mathrm{~m}$ & $\mathrm{~m}$ & $\mathrm{~m}$ & 4.47 & $\mathrm{~m}$ & $\mathrm{~m}$ & 3.7 & 3.5 & 4.3 & 4.6 & 4.6 & 4.4 & 4.3 & 4.3 & 4.4 & 4.7 & 5.3 & 5.5 & 4.9 & 5.3 & 6.2 & 6.3 & 6.1 & 5.8 & 5.5 & 5.7 & 5.7 & 5.8 & 5.8 & 5.8 & 5.8 \\
\hline land & $\mathrm{m}$ & $\mathrm{m}$ & $\mathrm{m}$ & $\mathrm{m}$ & $\mathrm{m}$ & $\mathrm{m}$ & $\mathrm{m}$ & $\mathrm{m}$ & $\mathrm{m}$ & $\mathrm{m}$ & 4.4 & 4.5 & 4.6 & 4.3 & 4.0 & 4.0 & 4.3 & 4.0 & 3.9 & 4.1 & 3.9 & 4.2 & 4.5 & 4.4 & 4.3 & 4.3 & 4.3 & 4.6 & 4.6 & 4.6 & 4.7 & 4.7 & 4.8 \\
\hline ortugal & 3.3 & 3.5 & 3.1 & 2.7 & 2.7 & 3.0 & 3.2 & 3.1 & 3.3 & 3.0 & 3.7 & 3.9 & 3.8 & 4.2 & 4.3 & 4.7 & 5.1 & 5.1 & 5.2 & 5.4 & 6.2 & 6.1 & 6.3 & 6.9 & 7.0 & 7.1 & 6.9 & 6.6 & 6.7 & 6.7 & 6.7 & 6.8 & 6.8 \\
\hline Slovak Rer & $\mathrm{m}$ & $\mathrm{m}$ & $\mathrm{m}$ & $\mathrm{m}$ & $\mathrm{m}$ & $\mathrm{m}$ & $\mathrm{m}$ & $\mathrm{m}$ & $\mathrm{m}$ & $\mathrm{m}$ & $\mathrm{m}$ & $\mathrm{m}$ & $\mathrm{m}$ & $\mathrm{m}$ & $\mathrm{m}$ & 5.7 & 5.5 & 5.3 & 5.2 & 5.2 & 4.9 & 4.9 & 5.0 & 5.1 & 5.3 & 5.2 & 5.0 & 5.2 & 5.2 & 5.3 & 5.4 & 5.4 & 5.5 \\
\hline Slovenia & $\mathrm{m}$ & $\mathrm{m}$ & $\mathrm{m}$ & $\mathrm{m}$ & $\mathrm{m}$ & $\mathrm{m}$ & $\mathrm{m}$ & $\mathrm{m}$ & $\mathrm{m}$ & $\mathrm{m}$ & $\mathrm{m}$ & $\mathrm{m}$ & $\mathrm{m}$ & $\mathrm{m}$ & $\mathrm{m}$ & $\mathrm{m}$ & 5.9 & 5.9 & 5.9 & 5.9 & 6.1 & 6.3 & 6.3 & 6.2 & 6.1 & 6.1 & 6.0 & 5.6 & 5.1 & 5.7 & 5.8 & 5.8 & 5.9 \\
\hline Spain & 4.2 & 4.3 & 4.4 & 4.8 & 4.5 & 4.3 & 4.2 & 4.3 & 4.7 & 4.8 & 5.1 & 5.2 & 5.5 & 5.7 & 5.5 & 5.4 & 5.4 & 5.3 & 5.3 & 5.3 & 5.2 & 5.2 & 5.2 & 5.7 & 5.8 & 5.8 & 6.0 & 6.1 & 6.1 & 6.1 & 6.2 & 6.2 & 6.2 \\
\hline Sweden & 8.3 & 8.3 & 8.4 & 8.3 & 8.1 & 7.7 & 7.4 & 7.4 & 7.3 & 7.3 & 7.4 & 7.1 & 7.1 & 6.7 & 6.3 & 6.2 & 6.4 & 6.2 & 6.3 & 6.3 & 6.3 & 6.6 & 6.8 & 6.9 & 6.7 & 6.7 & 6.6 & 6.6 & 6.6 & 6.6 & 6.7 & 6.7 & 6.7 \\
\hline itzerla & 3.6 & 3.7 & 3.7 & 4.0 & 3.8 & 3.9 & 4.0 & 4.1 & 4.1 & 4.3 & 3.9 & 4.3 & 4.5 & 4.6 & 4.6 & 4.6 & 4.9 & 4.9 & 4.9 & 5.0 & 5.0 & 5.4 & 5.7 & 5.9 & 5.9 & 6.0 & 5.7 & 5.6 & 5.5 & 5.8 & 5.6 & 5.4 & 5.2 \\
\hline Turkey & 0. & 1.0 & 1.1 & 1.1 & 0.9 & 0.8 & 0.8 & 0.8 & 1.2 & 1.4 & 1.6 & 1.8 & 1.9 & 1.8 & 1.9 & 1.8 & 2.0 & 2.2 & 2.6 & 2. & 3.0 & 3.2 & 3.3 & 3.4 & 3.6 & 3.7 & 4.0 & 4.1 & $m$ & $m$ & $m$ & $m$ & $m$ \\
\hline Ilean & 4. & 5.1 & 4.9 & 5.1 & 5.0 & 4.9 & 4.8 & 4.8 & 4.7 & 4.7 & 4.9 & 5.2 & 5.7 & 5. & 5.7 & 5.6 & 5.6 & 5.3 & 5.3 & 5.5 & 5.5 & 5.7 & 6.0 & 0.1 & 0.0 & 0.1 & 18 & 6.8 & 6.9 & 6.9 & 6.9 & 7.0 & 7.0 \\
\hline United States & 3.7 & 3.9 & 4.1 & 4.2 & 4.1 & 4.1 & 4.3 & 4.4 & 4.4 & 4.6 & 4.8 & 5.3 & 5.6 & 5.9 & 6.1 & 6.2 & 6.2 & 6.1 & 5.9 & 5.8 & 5.9 & 6.3 & 6.6 & 6.8 & 6.9 & 7.0 & 7.1 & 7.2 & 7.5 & 8.6 & 8.9 & 9.2 & 8.1 \\
\hline
\end{tabular}

Note: Social spending aggregates based on detailed data for 1980-2007; aggregate spending estimates for 2008-2012. 
DELSA/ELSA/WD/SEM(2011)9

Table A.I.1.4.d: Public social expenditures on other services as \% GDP, 1980 - 2012, estimated for 2008 to 2012

\begin{tabular}{|c|c|c|c|c|c|c|c|c|c|c|c|c|c|c|c|c|c|c|c|c|c|c|c|c|c|c|c|c|c|c|c|c|c|}
\hline & 1980 & 1981 & 1982 & 1983 & 1984 & 1985 & 1986 & 1987 & 1988 & 1989 & 1990 & 1991 & 1992 & 1993 & 1994 & 1995 & 1996 & 1997 & 1998 & 1999 & 2000 & 2001 & 2002 & 2003 & 2004 & 2005 & 2006 & 2007 & 2008 & 2009 & 2010 & 2011 & 2012 \\
\hline$\overline{\text { Australia }}$ & 0.5 & 0.4 & 0.6 & 0.7 & 0.7 & 1.0 & 0.9 & 1.5 & 1.5 & 1.5 & 1.6 & 1.9 & 2.3 & 2.3 & 2.4 & 2.5 & 2.3 & 2.2 & 2.9 & 2.8 & 2.9 & 2.8 & 3.1 & 3.2 & 3.2 & 3.1 & 2.9 & 2.9 & 3.2 & 3.7 & 3.0 & 2.9 & 2.7 \\
\hline Austria & 0.9 & $\mathrm{~m}$ & $\mathrm{~m}$ & $\mathrm{~m}$ & $\mathrm{~m}$ & 1.2 & $\mathrm{~m}$ & $\mathrm{~m}$ & $\mathrm{~m}$ & $\mathrm{~m}$ & 1.3 & 1.4 & 1.4 & 1.4 & 1.6 & 1.5 & 1.7 & 1.8 & 1.7 & 1.9 & 1.8 & 1.8 & 1.8 & 1.9 & 2.0 & 2.0 & 2.1 & 2.1 & 2.3 & 4.2 & 4.0 & 3.4 & 3.1 \\
\hline Belgium & 0.3 & 0.3 & 0.3 & 0.3 & 0.3 & 1.4 & 1.5 & 1.5 & 1.4 & 1.4 & 1.4 & 1.3 & 1.3 & 1.8 & 1.9 & 2.4 & 2.6 & 2.2 & 3.1 & 3.1 & 3.3 & 3.4 & 3.4 & 2.8 & 2.8 & 2.8 & 3.1 & 3.0 & 3.7 & 5.2 & 4.9 & 4.4 & 4.0 \\
\hline Canada & 2.8 & 2.8 & 3.2 & 3.3 & 3.2 & 3.7 & 3.4 & 3.3 & 3.2 & 3.3 & 3.7 & 4.2 & 4.6 & 4.7 & 4.5 & 4.2 & 3.8 & 3.5 & 3.8 & 3.4 & 3.4 & 3.3 & 3.2 & 3.2 & 3.3 & 3.3 & 3.3 & 3.2 & 3.2 & 3.8 & 3.6 & 3.4 & 3.2 \\
\hline Chile & $\mathrm{m}$ & $\mathrm{m}$ & $\mathrm{m}$ & $\mathrm{m}$ & $\mathrm{m}$ & $\mathrm{m}$ & $\mathrm{m}$ & $\mathrm{m}$ & $\mathrm{m}$ & $\mathrm{m}$ & $\mathrm{m}$ & $\mathrm{m}$ & $\mathrm{m}$ & $\mathrm{m}$ & $\mathrm{m}$ & 0.7 & 0.8 & 0.7 & 0.7 & 0.9 & 0.9 & 1.0 & 1.1 & 0.9 & 0.8 & 0.8 & 0.7 & 0.9 & 0.9 & 1.4 & 1.2 & $m$ & $m$ \\
\hline Czech Republic & $\mathrm{m}$ & $\mathrm{m}$ & $\mathrm{m}$ & $\mathrm{m}$ & $\mathrm{m}$ & $\mathrm{m}$ & $\mathrm{m}$ & $\mathrm{m}$ & $\mathrm{m}$ & $\mathrm{m}$ & 0.5 & 0.7 & 1.0 & 1.0 & 1.0 & 0.9 & 0.9 & 1.0 & 1.6 & 1.7 & 1.9 & 1.8 & 1.9 & 1.8 & 1.8 & 1.8 & 1.7 & 1.2 & 1.4 & 2.8 & 3.0 & 3.1 & 3.0 \\
\hline enmark & 4.8 & 5.0 & 5.1 & 4.9 & 4.7 & 4.8 & 5.5 & 5.6 & 5.7 & 5.7 & 6.3 & 6.5 & 6.7 & 7.3 & 7.7 & 8.0 & 7.9 & 7.7 & 7.3 & 7.6 & 7.4 & 7.5 & 7.7 & 7.7 & 7.6 & 7.5 & 7.4 & 7.0 & 7.5 & 10.0 & 9.7 & 9.3 & 8.8 \\
\hline stonia & $\mathrm{m}$ & $\mathrm{m}$ & $\mathrm{m}$ & $\mathrm{m}$ & $\mathrm{m}$ & $\mathrm{m}$ & $\mathrm{m}$ & $\mathrm{m}$ & $\mathrm{m}$ & $\mathrm{m}$ & $\mathrm{m}$ & $\mathrm{m}$ & $\mathrm{m}$ & $\mathrm{m}$ & $\mathrm{m}$ & $\mathrm{m}$ & $\mathrm{m}$ & $\mathrm{m}$ & $\mathrm{m}$ & $\mathrm{m}$ & 0.7 & 0.6 & 0.6 & 0.7 & 0.7 & 0.7 & 0.7 & 0.7 & 2.2 & 4.6 & 3.9 & 3.0 & 2.4 \\
\hline Finland & 2.7 & 2.7 & 2.6 & 3.1 & 3.1 & 3.2 & 3.2 & 3.3 & 3.4 & 3.4 & 3.7 & 4.6 & 5.2 & 5.2 & 5.2 & 5.0 & 5.3 & 4.9 & 4.6 & 4.6 & 4.2 & 4.2 & 4.3 & 4.5 & 4.6 & 4.6 & 4.7 & 4.6 & 4.9 & 7.5 & 6.9 & 6.1 & 5.5 \\
\hline France & 1.3 & 1.4 & 1.6 & 1.6 & 1.7 & 2.3 & 2.4 & 2.5 & 2.4 & 2.3 & 3.1 & 3.2 & 3.4 & 3.9 & 3.9 & 4.0 & 4.0 & 4.0 & 4.5 & 4.5 & 4.0 & 3.9 & 3.9 & 3.9 & 3.9 & 3.8 & 3.8 & 3.8 & 3.8 & 5.3 & 5.4 & 4.8 & 4.4 \\
\hline Germany & 0.7 & 0.8 & 0.8 & 0.8 & 0.8 & 1.3 & 1.5 & 1.7 & 1.7 & 1.6 & 1.9 & 2.7 & 3.2 & 3.2 & 3.0 & 3.0 & 3.1 & 2.8 & 2.8 & 2.9 & 2.8 & 2.8 & 2.9 & 2.9 & 2.8 & 2.9 & 2.8 & 2.7 & 2.7 & 4.8 & 4.6 & 3.8 & 3.2 \\
\hline Sreece & 0.1 & 0.1 & 0.1 & 0.2 & 0.2 & 0.4 & 0.4 & 0.4 & 0.3 & 0.3 & 1.1 & 1.1 & 1.2 & 1.3 & 1.2 & 1.3 & 1.4 & 1.5 & 1.5 & 1.7 & 1.8 & 1.9 & 1.7 & 1.5 & 1.6 & 1.5 & 1.6 & 1.6 & 2.8 & 4.5 & 3.1 & 3.0 & 2.5 \\
\hline Hungary & $\mathrm{m}$ & $\mathrm{m}$ & $\mathrm{m}$ & $\mathrm{m}$ & $\mathrm{m}$ & $\mathrm{m}$ & $\mathrm{m}$ & $\mathrm{m}$ & $\mathrm{m}$ & $\mathrm{m}$ & $\mathrm{m}$ & $\mathrm{m}$ & $\mathrm{m}$ & $\mathrm{m}$ & $\mathrm{m}$ & $\mathrm{m}$ & $\mathrm{m}$ & $\mathrm{m}$ & $\mathrm{m}$ & 3.0 & 2.9 & 2.9 & 3.1 & 3.1 & 2.8 & 3.0 & 3.0 & 3.2 & 3.5 & 4.1 & 3.3 & 3.0 & 2.3 \\
\hline Iceland & $\mathrm{m}$ & $\mathrm{m}$ & $\mathrm{m}$ & $\mathrm{m}$ & $\mathrm{m}$ & $\mathrm{m}$ & $\mathrm{m}$ & $\mathrm{m}$ & $\mathrm{m}$ & $\mathrm{m}$ & 2.5 & 2.6 & 2.8 & 2.9 & 3.0 & 3.1 & 3.1 & 3.2 & 3.4 & 3.4 & 3.6 & 3.8 & 4.3 & 4.7 & 5.0 & 4.9 & 5.0 & 32 & 3.3 & 3.5 & 3.0 & 2.8 & 2.6 \\
\hline Ireland & 1.3 & 1.3 & 1.3 & 1.5 & 1.4 & 2.6 & 2.6 & 2.6 & 2.5 & 2.2 & 1.9 & 1.8 & 2.0 & 2.0 & 2.2 & 2.2 & 2.1 & 2.1 & 1.9 & 1.9 & 1.7 & 1.8 & 1.8 & 1.8 & 1.7 & 1.7 & 1.6 & 1.6 & 3.1 & 5.2 & 5.2 & 4.2 & 3.3 \\
\hline |srael & $\mathrm{m}$ & $\mathrm{m}$ & $\mathrm{m}$ & $\mathrm{m}$ & $\mathrm{m}$ & $\mathrm{m}$ & $\mathrm{m}$ & $\mathrm{m}$ & $\mathrm{m}$ & $\mathrm{m}$ & $\mathrm{m}$ & $\mathrm{m}$ & $\mathrm{m}$ & $\mathrm{m}$ & $\mathrm{m}$ & 2.2 & 2.3 & 2.4 & 2.4 & 2.4 & 2.4 & 2.6 & 2.7 & 2.6 & 2.5 & 2.4 & 2.4 & 2.3 & 2.4 & 2.5 & 2.5 & 2.5 & 2.5 \\
\hline Italy & 0.3 & 0.3 & 0.3 & 0.3 & 0.3 & 0.3 & 0.3 & 0.3 & 0.3 & 0.3 & 0.6 & 0.7 & 0.6 & 0.6 & 0.6 & 0.6 & 0.7 & 0.7 & 1.2 & 1.3 & 1.4 & 1.5 & 1.5 & 1.7 & 1.5 & 1.5 & 1.5 & 1.4 & 2.1 & 3.7 & 3.7 & 3.2 & 2.6 \\
\hline Japan & 0.5 & 0.5 & 0.5 & 0.4 & 0.4 & 0.4 & 0.4 & 0.4 & 0.4 & 0.4 & 0.7 & 0.8 & 0.8 & 0.9 & 1.0 & 1.0 & 1.0 & 1.0 & 1.1 & 1.2 & 1.6 & 1.8 & 1.9 & 2.0 & 2.1 & 2.1 & 2.0 & 2.0 & 2.3 & $m$ & $m$ & $m$ & $m$ \\
\hline Luxembou & 0.6 & 1.2 & 1.1 & 1.5 & 1.0 & 0.9 & 0.8 & 0.8 & 0.9 & 0.9 & 1.0 & 0.9 & 1.0 & 0.9 & 1.0 & 1.0 & 1.1 & 1.1 & 1.1 & 1.4 & 1.4 & 1.7 & 1.8 & 2.0 & 2.2 & 2.3 & 2.2 & 2.1 & 2.3 & 4.6 & 4.0 & 3.9 & 3.9 \\
\hline Mexico & $\mathrm{m}$ & $\mathrm{m}$ & $\mathrm{m}$ & $\mathrm{m}$ & $\mathrm{m}$ & 0.6 & 0.6 & 0.5 & 0.6 & 0.7 & 0.9 & 1.0 & 1.1 & 1.2 & 1.2 & 1.0 & 1.1 & 1.0 & 1.1 & 1.5 & 1.6 & 1.6 & 1.8 & 2.0 & 1.8 & 1.9 & 2.2 & 2.3 & 2.7 & 2.8 & 2.9 & 2.8 & m \\
\hline Netherland & 1.9 & 2.0 & 2.1 & 2.2 & 1.9 & 2.5 & 2.6 & 2.7 & 2.6 & 2.7 & 2.6 & 2.6 & 2.6 & 2.7 & 3.1 & 2.9 & 2.8 & 3.1 & 3.8 & 3.5 & 3.6 & 3.5 & 3.8 & 3.8 & 3.9 & 3.8 & 3.9 & 4.1 & 4.1 & 5.8 & 5.8 & 5.3 & 4.6 \\
\hline New Zeala & 0.7 & 0.7 & 0.8 & 0.9 & 0.9 & 1.1 & 1.0 & 1.0 & 1.2 & 1.2 & 1.2 & 1.2 & 1.7 & 1.5 & 1.6 & 1.6 & 1.7 & 1.8 & 2.0 & 2.0 & 2.0 & 1.9 & 1.8 & 1.8 & 1.8 & 1.9 & 1.9 & 1.9 & 2.1 & 2.3 & 2.1 & 2.1 & 1.9 \\
\hline Norway & 2.4 & $\mathrm{~m}$ & $\mathrm{~m}$ & $\mathrm{~m}$ & $\mathrm{~m}$ & 2.9 & $\mathrm{~m}$ & $\mathrm{~m}$ & 4.9 & 5.4 & 4.9 & 5.2 & 5.7 & 5.9 & 6.2 & 6.1 & 5.8 & 5.4 & 5.7 & 5.7 & 5.2 & 5.5 & 5.2 & 5.4 & 5.4 & 5.0 & 4.8 & 5.0 & 4.9 & 7.2 & 7.1 & 5.9 & 5.5 \\
\hline Poland & $\mathrm{m}$ & $\mathrm{m}$ & $\mathrm{m}$ & $\mathrm{m}$ & $\mathrm{m}$ & $\mathrm{m}$ & $\mathrm{m}$ & $\mathrm{m}$ & $\mathrm{m}$ & $\mathrm{m}$ & 0.5 & 0.9 & 1.1 & 1.4 & 1.1 & 1.1 & 1.3 & 1.2 & 1.4 & 1.2 & 0.8 & 0.8 & 0.8 & 1.0 & 0.9 & 1.0 & 1.0 & 1.1 & 1.4 & 3.0 & 3.5 & 3.3 & 3.2 \\
\hline Portugal & 0.0 & 0.0 & 0.0 & 0.0 & 0.0 & 0.0 & 0.2 & 0.3 & 0.3 & 0.4 & 0.7 & 0.7 & 0.8 & 0.8 & 0.7 & 0.7 & 0.7 & 0.7 & 1.0 & 1.0 & 1.1 & 1.2 & 1.7 & 1.9 & 1.3 & 1.3 & 1.2 & 1.1 & 1.3 & 3.4 & 3.4 & 2.4 & 2.5 \\
\hline Slovak Republic & $\mathrm{m}$ & $\mathrm{m}$ & $\mathrm{m}$ & $\mathrm{m}$ & $\mathrm{m}$ & $\mathrm{m}$ & $\mathrm{m}$ & $\mathrm{m}$ & $\mathrm{m}$ & $\mathrm{m}$ & $\mathrm{m}$ & $\mathrm{m}$ & $\mathrm{m}$ & $\mathrm{m}$ & $\mathrm{m}$ & 1.2 & 1.2 & 1.2 & 0.9 & 1.2 & 1.2 & 1.3 & 1.4 & 1.3 & 1.3 & 1.3 & 1.3 & 1.1 & 1.3 & 3.2 & 3.0 & 2.4 & 1.9 \\
\hline Slovenia & $\mathrm{m}$ & $\mathrm{m}$ & $\mathrm{m}$ & $\mathrm{m}$ & $\mathrm{m}$ & $\mathrm{m}$ & $\mathrm{m}$ & $\mathrm{m}$ & $\mathrm{m}$ & $\mathrm{m}$ & $\mathrm{m}$ & $\mathrm{m}$ & $\mathrm{m}$ & $\mathrm{m}$ & $\mathrm{m}$ & $\mathrm{m}$ & 1.1 & 1.1 & 1.1 & 1.1 & 1.1 & 1.1 & 1.0 & 1.3 & 1.2 & 1.3 & 1.2 & 1.1 & 1.5 & 3.7 & 3.7 & 3.6 & 3.2 \\
\hline Spain & 0.3 & 0.4 & 0.4 & 0.4 & 0.4 & 0.5 & 0.8 & 0.8 & 1.0 & 1.1 & 1.2 & 1.1 & 1.1 & 1.0 & 0.9 & 1.1 & 1.2 & 1.2 & 1.7 & 2.0 & 2.1 & 2.0 & 2.1 & 2.1 & 2.2 & 2.3 & 2.4 & 2.4 & 3.3 & 5.4 & 5.3 & 4.6 & 4.1 \\
\hline Sweden & 5.0 & 5.0 & 4.8 & 5.0 & 4.9 & 6.8 & 6.9 & 6.8 & 6.8 & 6.3 & 6.7 & 7.6 & 9.3 & 10.3 & 10.2 & 9.1 & 9.2 & 8.8 & 9.0 & 8.8 & 8.1 & 8.1 & 8.3 & 8.2 & 8.1 & 8.2 & 8.3 & 8.0 & 8.0 & 9.7 & 8.6 & 7.8 & 7.3 \\
\hline Switzerland & 0.5 & 0.5 & 0.5 & 0.6 & 0.5 & 0.7 & 0.7 & 0.7 & 0.8 & 0.8 & 1.1 & 1.2 & 1.4 & 1.6 & 1.7 & 1.7 & 1.8 & 2.1 & 2.2 & 2.1 & 2.0 & 2.1 & 2.2 & 2.4 & 2.5 & 2.5 & 2.3 & 2.2 & 2.0 & 2.8 & 3.0 & 2.7 & 2.3 \\
\hline Turkey & $\mathrm{m}$ & $\mathrm{m}$ & $\mathrm{m}$ & $\mathrm{m}$ & $\mathrm{m}$ & $\mathrm{m}$ & $\mathrm{m}$ & $\mathrm{m}$ & $\mathrm{m}$ & $\mathrm{m}$ & $\mathrm{m}$ & $\mathrm{m}$ & $\mathrm{m}$ & $\mathrm{m}$ & $\mathrm{m}$ & $\mathrm{m}$ & $\mathrm{m}$ & $\mathrm{m}$ & $m$ & $\mathrm{~m}$ & $\mathrm{~m}$ & $\mathrm{~m}$ & $\mathrm{~m}$ & $\mathrm{~m}$ & $\mathrm{~m}$ & $\mathrm{~m}$ & $\mathrm{~m}$ & $\mathrm{~m}$ & $m$ & $m$ & $m$ & $m$ & m \\
\hline United Ki & 1.7 & 1.8 & 2.0 & 2.5 & 2.5 & 2.9 & 3.0 & 3.0 & 2.6 & 2.6 & 2.9 & 2.9 & 3.3 & 3.6 & 3.6 & 3.6 & 3.6 & 3.5 & 4.0 & 3.4 & 3.4 & 3.5 & 3.6 & 3.8 & 3.9 & 3.8 & 3.7 & 3.8 & 4.5 & 6.5 & 6.5 & 5.8 & 5.1 \\
\hline United States & 0.9 & 0.9 & 0.8 & 0.8 & 0.7 & 1.0 & 0.9 & 0.9 & 0.8 & 0.8 & 0.8 & 0.9 & 0.9 & 1.0 & 0.9 & 0.9 & 0.8 & 0.8 & 1.1 & 1.0 & 1.0 & 1.0 & 1.1 & 1.0 & 1.0 & 1.0 & 1.0 & 1.0 & 1.1 & 1.3 & 1.5 & 1.5 & 1.5 \\
\hline
\end{tabular}

Note: Social spending aggregates based on detailed data for 1980-2007; aggregate spending estimates for 2008-2012. 


\section{DELSA/ELSA/WD/SEM(2011)9}

Table A.I.1.5: Public social expenditures as \% of Trend GDP, 1980 - 2012, estimated for 2008 to 2012

\begin{tabular}{|c|c|c|c|c|c|c|c|c|c|c|c|c|c|c|c|c|c|c|c|c|c|c|c|c|c|c|c|c|c|c|c|c|c|}
\hline & 1980 & 1981 & 1982 & 1983 & 1984 & 1985 & 1986 & 1987 & 1988 & 1989 & 1990 & 1991 & 1992 & 1993 & 1994 & 1995 & 1996 & 1997 & 1998 & 1999 & 2000 & 2001 & 2002 & 2003 & 2004 & 2005 & 2006 & 2007 & 2008 & 2009 & 2010 & 2011 & 2012 \\
\hline$\overline{\text { Australia }}$ & 111.1 & 111.1 & 111.3 & 11.9 & 12.4 & 12.8 & 12.6 & 13.1 & 12.4 & 12.4 & 13.3 & 14.0 & 15.2 & 15.6 & 15.6 & 16.6 & 16.5 & 16.4 & 17.3 & 17.3 & 17.9 & 17.3 & 17.5 & 18.0 & 17.8 & 17.3 & 16.9 & 16.9 & \begin{tabular}{|l|l}
16.8 \\
\end{tabular} & 18.0 & 16.5 & 16.2 & 16.2 \\
\hline Austria & 22.8 & $\mathrm{~m}$ & $\mathrm{~m}$ & $\mathrm{~m}$ & $\mathrm{~m}$ & 23.2 & $\mathrm{~m}$ & $\mathrm{~m}$ & $\mathrm{~m}$ & $\mathrm{~m}$ & 24.4 & 24.7 & 25.1 & 25.9 & 26.6 & 26.3 & 26.5 & 26.5 & 26.7 & 27.5 & 27.4 & 27.1 & 27.0 & 27.2 & 27.2 & 27.1 & 27.1 & 27.0 & 27.4 & 28.3 & 28.1 & 27.8 & 27.7 \\
\hline Belgium & 23.9 & 24.9 & 25.2 & 25.0 & 24.4 & 25.2 & 25.1 & 25.0 & 25.4 & 24.8 & 25.3 & 25.9 & 25.9 & 26.2 & 26.0 & 26.0 & 26.3 & 25.7 & 25.8 & 26.0 & 25.8 & 25.9 & 26.0 & 25.9 & 26.6 & 26.4 & 26.5 & 26.6 & 27.4 & 28.5 & 28.4 & 28.3 & 28.2 \\
\hline anada & 14.1 & 14.2 & 15.8 & 16.1 & 16.4 & 17.3 & 17.4 & 17.3 & 17.3 & 17.5 & 18.3 & 19.7 & 20.2 & 20.4 & 19.7 & 18.8 & 17.8 & 17.3 & 17.9 & 17.3 & 17.0 & 17.3 & 17.5 & 17.5 & 17.4 & 17.4 & 17.3 & 17.2 & 17.3 & 18.4 & 18.4 & 18.5 & 18.7 \\
\hline $\operatorname{ech} \mathrm{F}$ & $\mathrm{m}$ & $\mathrm{m}$ & $\mathrm{m}$ & $\mathrm{m}$ & $\mathrm{m}$ & $\mathrm{m}$ & $\mathrm{m}$ & $\mathrm{m}$ & $\mathrm{m}$ & $\mathrm{m}$ & $\mathrm{m}$ & $\mathrm{m}$ & $\mathrm{m}$ & $\mathrm{m}$ & 17.8 & 18.5 & 18.7 & 19.0 & 18.8 & 19.1 & 19.7 & 19.6 & 20.2 & 20.3 & 19.3 & 19.5 & 19.4 & 19.4 & 19.3 & 19.8 & 20.0 & 20.1 & 20.0 \\
\hline nmark & 24.5 & 23.9 & 24.3 & 24.6 & 23.9 & 23.8 & 24.1 & 24.3 & 25.0 & 25.0 & 24.7 & 25.4 & 25.9 & 27.1 & 29.3 & 28.9 & 28.2 & 27.4 & 26.6 & 26.6 & 26.2 & 26.4 & 26.6 & 27.5 & 27.6 & 27.3 & 27.1 & 26.6 & 26.5 & 28.2 & 28.4 & 28.5 & 28.3 \\
\hline tonia & $\mathrm{m}$ & $\mathrm{m}$ & $\mathrm{m}$ & $\mathrm{m}$ & $\mathrm{m}$ & $\mathrm{m}$ & $\mathrm{m}$ & $\mathrm{m}$ & $\mathrm{m}$ & $\mathrm{m}$ & $\mathrm{m}$ & $\mathrm{m}$ & $\mathrm{m}$ & $\mathrm{m}$ & $\mathrm{m}$ & $\mathrm{m}$ & $\mathrm{m}$ & $\mathrm{m}$ & $\mathrm{m}$ & $\mathrm{m}$ & 14.1 & 13.1 & 12.9 & 13.1 & 13.7 & 13.8 & 14.3 & 15.1 & 16.7 & 17.9 & 18.2 & 17.6 & 16.9 \\
\hline land & 18.4 & 18.4 & 19.4 & 20.3 & 21.3 & 22.5 & 23.0 & 23.9 & 23.8 & 24.1 & 25.4 & 28.4 & 30.8 & 30.5 & 30.8 & 29.5 & 29.7 & 28.4 & 26.4 & 25.9 & 24.8 & 24.4 & 24.7 & 25.3 & 25.7 & 25.8 & 26.0 & 25.7 & 25.9 & 27.0 & 26.9 & 26.9 & 26.9 \\
\hline ance & 21.1 & 21.7 & 22.2 & 22.2 & 22.3 & 25.3 & 25.3 & 25.5 & 25.8 & 25.3 & 25.4 & 25.8 & 26.5 & 27.4 & 27.5 & 28.3 & 28.4 & 28.3 & 28.8 & 29.1 & 28.2 & 28.0 & 28.3 & 28.7 & 29.0 & 28.9 & 28.7 & 28.6 & 28.4 & 29.4 & 29.7 & 29.4 & 29.0 \\
\hline ermany & $\mathrm{m}$ & $\mathrm{m}$ & $\mathrm{m}$ & $\mathrm{m}$ & $\mathrm{m}$ & $\mathrm{m}$ & $\mathrm{m}$ & $\mathrm{m}$ & $\mathrm{m}$ & $\mathrm{m}$ & $\mathrm{m}$ & 24.2 & 26.0 & 25.9 & 26.2 & 26.7 & 27.1 & 26.5 & 26.4 & 26.6 & 26.8 & 26.9 & 27.2 & 27.1 & 26.6 & 26.7 & 26.2 & 25.6 & 25.5 & 26.3 & 26.6 & 26.3 & 25.9 \\
\hline eece & 10.5 & 12.3 & 14.1 & 14.4 & 14.9 & 15.8 & 15.6 & 15.1 & 14.5 & 15.7 & 16.5 & 16.1 & 16.1 & 16.4 & 16.5 & 16.9 & 17.4 & 17.6 & 18.3 & 18.8 & 18.9 & 20.2 & 19.7 & 19.7 & 19.8 & 20.6 & 21.2 & 21.6 & 22.7 & 23.7 & 21.2 & 20.6 & 20.3 \\
\hline ngary & $\mathrm{m}$ & $\mathrm{m}$ & $\mathrm{m}$ & $\mathrm{m}$ & $\mathrm{m}$ & $\mathrm{m}$ & $\mathrm{m}$ & $\mathrm{m}$ & $\mathrm{m}$ & $\mathrm{m}$ & $\mathrm{m}$ & $\mathrm{m}$ & $\mathrm{m}$ & $\mathrm{m}$ & $\mathrm{m}$ & $\mathrm{m}$ & $\mathrm{m}$ & $\mathrm{m}$ & $\mathrm{m}$ & 21.0 & 20.4 & 20.1 & 21.4 & 22.5 & 22.1 & 23.1 & 23.5 & 23.2 & 23.6 & 22.6 & 21.9 & 21.9 & 21.3 \\
\hline land & $\mathrm{m}$ & $\mathrm{m}$ & $\mathrm{m}$ & $\mathrm{m}$ & $\mathrm{m}$ & $\mathrm{m}$ & $\mathrm{m}$ & $\mathrm{m}$ & $\mathrm{m}$ & $\mathrm{m}$ & 13.9 & 14.2 & 14.0 & 14.3 & 14.5 & 14.5 & 14.6 & 14.5 & 15.0 & 15.6 & 15.5 & 15.5 & 16.5 & 17.2 & 17.5 & 17.1 & 16.4 & 15.3 & 15.3 & 16.9 & 14.6 & 14.0 & 13.4 \\
\hline and & 17.1 & 17.2 & 17.6 & 17.1 & 16.7 & 21.1 & 20.5 & 20.0 & 19.0 & 17.8 & 15.5 & 15.8 & 16.1 & 15.7 & 15.4 & 15.3 & 14.5 & 14.2 & 13.1 & 14.5 & 13.9 & 14.7 & 15.6 & 16.0 & 16.3 & 16.2 & 16.3 & 17.1 & 18.5 & 20.3 & 20.5 & 19.2 & 18.2 \\
\hline ael & $\mathrm{m}$ & $\mathrm{m}$ & $\mathrm{m}$ & $\mathrm{m}$ & $\mathrm{m}$ & $\mathrm{m}$ & $\mathrm{m}$ & $\mathrm{m}$ & $\mathrm{m}$ & $\mathrm{m}$ & $\mathrm{m}$ & $\mathrm{m}$ & $\mathrm{m}$ & $\mathrm{m}$ & $\mathrm{m}$ & $\mathrm{m}$ & $\mathrm{m}$ & $\mathrm{m}$ & $\mathrm{m}$ & 17.3 & 18.0 & 18.7 & 18.3 & 17.6 & 16.7 & 16.2 & 16.0 & 15.8 & 16.1 & 16.0 & 16.1 & 16.1 & 15.8 \\
\hline Italy & 18.4 & 19.5 & 19.5 & 20.2 & 19.8 & 20.3 & 20.5 & 20.9 & 21.3 & 21.6 & 20.2 & 20.4 & 20.7 & 20.4 & 20.4 & 19.7 & 21.7 & 22.5 & 22.7 & 23.0 & 23.4 & 23.8 & 24.0 & 24.2 & 24.7 & 25.0 & 25.5 & 25. & 25.7 & 26.2 & 26.5 & 26.3 & 26.0 \\
\hline apar & 10.6 & 11.0 & 11.2 & 11.5 & 11.3 & 11.3 & 11.6 & 11.7 & 11.7 & 11.6 & 11.9 & 12.0 & 12.3 & 12.8 & 13.4 & 14.3 & 14.8 & 14.8 & 15.1 & 15.7 & 16.3 & 17.0 & 17.3 & 17.7 & 18.0 & 18.6 & 18.7 & 19.1 & 19.8 & $m$ & $m$ & $m$ & $m$ \\
\hline rea & $\mathrm{m}$ & $\mathrm{m}$ & $\mathrm{m}$ & $\mathrm{m}$ & $\mathrm{m}$ & $\mathrm{m}$ & $\mathrm{m}$ & $\mathrm{m}$ & $\mathrm{m}$ & $\mathrm{m}$ & 2.9 & 2.8 & 3.0 & 3.0 & 3.1 & 3.3 & 3.5 & 3.8 & 4.7 & 6.0 & 4.8 & 5.2 & 5.2 & 5.4 & 6.0 & 6.5 & 7.5 & 7.7 & 8.1 & 8.7 & 8.9 & 9.3 & 9.7 \\
\hline xxembour & 20.8 & 21.6 & 20.5 & 20.6 & 19.7 & 19.3 & 19.4 & 20.0 & 19.7 & 19.7 & 19.8 & 20.7 & 20.4 & 20.6 & 20.2 & 20.3 & 19.8 & 20.3 & 20.4 & 20.5 & 20.5 & 21.1 & 22.4 & 23.1 & 23.8 & 23.2 & 22.2 & 21.6 & 21.6 & 23.0 & 22.2 & 22.3 & 22.6 \\
\hline therlands & 25.2 & 25.5 & 26.2 & 26.7 & 25.8 & 25.2 & 24.7 & 24.4 & 24.1 & 24.1 & 26.0 & 25.8 & 26.0 & 25.7 & 24.3 & 23.6 & 22.5 & 22.0 & 21.7 & 21.0 & 20.3 & 20.0 & 20.3 & 20.7 & 20.8 & 20.4 & 20.4 & 20.5 & 20.6 & 21.8 & 22.0 & 21.9 & 21.3 \\
\hline New Zeal & 18.0 & 18.3 & 19.0 & 18.7 & 18.3 & 18.4 & 18.9 & 19.3 & 19.9 & 21.2 & 21.0 & 21.0 & 21.0 & 20.0 & 19.7 & 19.2 & 19.0 & 19.8 & 19.8 & 19.6 & 19.3 & 18.7 & 19.0 & 18.7 & 18.3 & 18.8 & 19.3 & 18.9 & 19.9 & 20.7 & 21.1 & 21.4 & 21.7 \\
\hline rway & 20.5 & $\mathrm{~m}$ & $\mathrm{~m}$ & $\mathrm{~m}$ & $\mathrm{~m}$ & 22.8 & $\mathrm{~m}$ & $\mathrm{~m}$ & 23.0 & 24.4 & 25.4 & 26.8 & 27.7 & 27.9 & 27.8 & 27.2 & 27.5 & 27.3 & 27.7 & 28.3 & 28.6 & 29.0 & 29.4 & 30.1 & 29.9 & 29.3 & 28.3 & 28.0 & 28.8 & 30.1 & 30.3 & 30.3 & 29.8 \\
\hline land & $\mathrm{m}$ & $\mathrm{m}$ & $\mathrm{m}$ & $\mathrm{m}$ & $\mathrm{m}$ & $\mathrm{m}$ & $\mathrm{m}$ & $\mathrm{m}$ & $\mathrm{m}$ & $\mathrm{m}$ & $\mathrm{m}$ & $\mathrm{m}$ & $\mathrm{m}$ & $\mathrm{m}$ & & 21.9 & 22.4 & 22.3 & 21.5 & 22.0 & 20.9 & 21.8 & 21.7 & 21.8 & 21.3 & 20.7 & 20.7 & 19.9 & 20.2 & 21.2 & 21.7 & 21.5 & 21.4 \\
\hline rtugal & 10.2 & 10.8 & 10.0 & 9.8 & 9.2 & 9.3 & 10.1 & 10.7 & 11.3 & 11.2 & 13.2 & 14.2 & 14.6 & 15.3 & 15.2 & 16.2 & 16.9 & 16.9 & 17.5 & 18.0 & 19.4 & 19.6 & 20.7 & 21.9 & 22.1 & 22.5 & 22.6 & 22.5 & 22.8 & 24.7 & 25.2 & 23.8 & 23.3 \\
\hline ovak & $\mathrm{m}$ & $\mathrm{m}$ & $\mathrm{m}$ & $\mathrm{m}$ & $\mathrm{m}$ & $\mathrm{m}$ & $\mathrm{m}$ & $\mathrm{m}$ & $\mathrm{m}$ & $\mathrm{m}$ & $\mathrm{m}$ & $\mathrm{m}$ & $\mathrm{m}$ & $\mathrm{m}$ & $\mathrm{m}$ & 18.6 & 18.7 & 18.6 & 18.8 & 18.7 & 17.5 & 17.2 & 17.3 & 16.6 & 16.0 & 16.0 & 16.0 & 16.4 & 16.8 & 17.9 & 17.7 & 17.1 & 16.6 \\
\hline ovenia & $\mathrm{m}$ & $\mathrm{m}$ & $\mathrm{m}$ & $\mathrm{m}$ & $\mathrm{m}$ & $\mathrm{m}$ & $\mathrm{m}$ & $\mathrm{m}$ & $\mathrm{m}$ & $\mathrm{m}$ & $\mathrm{m}$ & $\mathrm{m}$ & $\mathrm{m}$ & $\mathrm{m}$ & $\mathrm{m}$ & $\mathrm{m}$ & $\mathrm{m}$ & $\mathrm{m}$ & $\mathrm{m}$ & 23.1 & 23.0 & 22.8 & 22.9 & 22.2 & 22.0 & 22.0 & 22.2 & 21.7 & 22.5 & 23.2 & 23.2 & 23.4 & 23.3 \\
\hline Spain & 15.1 & 15.9 & 15.8 & 16.5 & 16.3 & 17.0 & 16.9 & 17.2 & 18.1 & 18.5 & 20.5 & 21.2 & 21.8 & 22.3 & 21.3 & 20.7 & 20.6 & 20.2 & 20.4 & 20.5 & 20.8 & 20.5 & 20.5 & 21.0 & 21.1 & 21.4 & 21.4 & 21.6 & 22.7 & 24.5 & 24.7 & 24.0 & 23.5 \\
\hline veden & 27.2 & 27.5 & 27.4 & 27.7 & 27.5 & 29.8 & 30.0 & 30.5 & 31.1 & 30.5 & 31.0 & 31.7 & 33.8 & 33.7 & 33.1 & 31.5 & 30.8 & 29.8 & 30.1 & 29.8 & 28.9 & 28.7 & 29.3 & 30.0 & 29.9 & 29.6 & 29.5 & 28.5 & 27.5 & 27.7 & 27.2 & 26.9 & 26.4 \\
\hline Switzerlan & $\mathrm{m}$ & 13.7 & 14.0 & 14.3 & 14.7 & 14.8 & 14.9 & 14.9 & 15.1 & 15.1 & 14.1 & 14.7 & 16.0 & 17.2 & 17.2 & 17.2 & 17.7 & 18.5 & 18.8 & 18.5 & 18.1 & 18.5 & 19.0 & 19.7 & 19.8 & 19.9 & 19.3 & 18.9 & 18.4 & 19.1 & 19.3 & 18.9 & 18.5 \\
\hline rkey & $\mathrm{m}$ & $\mathrm{m}$ & $\mathrm{m}$ & $\mathrm{m}$ & $\mathrm{m}$ & $\mathrm{m}$ & $\mathrm{m}$ & $\mathrm{m}$ & $\mathrm{m}$ & $\mathrm{m}$ & $\mathrm{m}$ & $\mathrm{m}$ & $\mathrm{m}$ & $\mathrm{m}$ & $\mathrm{m}$ & $\mathrm{m}$ & $\mathrm{m}$ & $\mathrm{m}$ & $\mathrm{m}$ & $\mathrm{m}$ & $\mathrm{m}$ & $\mathrm{m}$ & $\mathrm{m}$ & $\mathrm{m}$ & $\mathrm{m}$ & $\mathrm{m}$ & $\mathrm{m}$ & $\mathrm{m}$ & $m$ & $\mathrm{~m}$ & $m$ & $m$ & $m$ \\
\hline United King & 16.7 & 17.7 & 18.2 & 19.2 & 19.3 & 19.6 & 20.0 & 19.7 & 18.7 & 17.9 & 17.3 & 18.1 & 19.5 & 20.2 & 20.2 & 20.2 & 19.9 & 19.0 & 19.2 & 18.9 & 19.0 & 19.7 & 19.7 & 20.2 & 20.9 & 21.1 & 21.1 & 21.2 & 21.6 & 23.0 & 23.3 & 22.7 & 22.1 \\
\hline United States & 12.7 & 12.8 & 12.8 & 13.1 & 12.7 & 12.8 & 12.9 & 12.9 & 12.9 & 13.0 & 13.2 & 13.9 & 14.5 & 14.8 & 14.9 & 14.9 & 14.8 & 14.5 & 14.6 & 14.4 & 14.5 & 15.1 & 15.5 & 15.6 & 15.7 & 15.7 & 15.9 & 16.1 & 16.5 & 18.5 & 19.6 & 19.6 & 19.0 \\
\hline
\end{tabular}


ANNEX I.2: ADDITIONAL NET SOCIAL EXPENDITURE INDICATORS:

A.I.2.1: Individual country data : 2001, 2003, 2005 and 2007 
DELSA/ELSA/WD/SEM(2011)9

Table Annex I.A.2.1

Detailed information on the impact of the tax system on social expenditure

\section{AUSTRALIA}

A. Average Itemised Tax Rates (AITR \%)

\begin{tabular}{|c|c|c|c|c|c|}
\hline & & 2001 & 2003 & 2005 & 2007 \\
\hline \multirow[t]{4}{*}{1} & $\begin{array}{l}\text { Old-age cash benefits } \\
\text { - public pensions }\end{array}$ & & & & \\
\hline & Age Pension & 0.38 & 0.03 & 0.84 & 0.12 \\
\hline & Wife's Pension & 0.38 & 1.25 & 0.14 & 0.07 \\
\hline & Widow's B Pension & 0.26 & 0.77 & 0.67 & 0.67 \\
\hline \multirow{4}{*}{$\begin{array}{l}1 \mathrm{~b} \\
1 \mathrm{c}\end{array}$} & - early retirement benefits & & & & \\
\hline & - private pensions & & & & \\
\hline & Superannuation pension & 16.83 & 15.07 & 13.58 & 13.30 \\
\hline & Superannuation Lump Sums & 2.66 & 10.45 & 9.50 & 20.80 \\
\hline \multirow{3}{*}{2 a } & Survivors' benefits & & & & \\
\hline & - public pensions & & & & \\
\hline & Veteran's Service Pensions & 0.68 & 0.00 & 0.54 & 0.21 \\
\hline \multirow{3}{*}{$3 \mathrm{c}$} & Incapacity-related benefits & & & & \\
\hline & - Sickness payments & & & & \\
\hline & Sickness Allowance & 0.45 & 0.09 & 0.05 & 0.00 \\
\hline 4 & Family cash benefits & & & & \\
\hline \multirow{4}{*}{$4 a$} & - Family benefits & & & & \\
\hline & Parenting Allowance & 1.03 & 1.49 & 1.44 & 0.24 \\
\hline & Partner Allowance & 0.17 & 1.02 & 0.61 & 0.14 \\
\hline & Carer's Payment & 0.00 & 0.19 & 0.31 & 0.00 \\
\hline \multirow[t]{2}{*}{$4 c$} & - Sole parent benefits & & & & \\
\hline & Sole Parent & 0.64 & 0.92 & 1.43 & 0.72 \\
\hline \multirow{3}{*}{6} & Unemployment & & & & \\
\hline & - unemployment assistance benefit & & & & \\
\hline & Unemployment Benefits & 1.10 & 0.81 & 1.11 & 0.33 \\
\hline 9 & Wage income & 23.59 & 24.63 & 23.78 & 22.48 \\
\hline
\end{tabular}

The AITRs for wage income, superannuation pensions and superannuation lump sums were calculated using a sample file of Australian tax returns in 2001. All other AITRs were calculated using the STINMOD model, a static microsimulation model developed by the National Center for Social and Economic Modelling (NATSEM).

The AITRs were obtained by calculating the amount of tax paid in aggregate with and without the income streams. The difference between the taxes paid was then divided by the value of the income stream to reveal the value of the AITR.

Sources: STINMOD distributional model. Revenue Group of The Treasury, Australian Government.

B. Average implicit indirect tax rates of consumption out of benefit income

Indirect taxes paid out of consumption of cash transfers, in millions of Australian dollars

\begin{tabular}{|c|c|c|c|c|c|}
\hline & & 2001 & 2003 & 2005 & 2007 \\
\hline (1) & Private final consumption expenditure & 444473 & 504948 & 565338 & 655287 \\
\hline (2) & Private consumption plus Government consumption minus Government wages & 509009 & 581609 & 658600 & 772490 \\
\hline \multirow[t]{2}{*}{ (3) } & General consumption taxes plus excise duties $(5110+5121)$ & 48674 & 57029 & 62895 & 69843 \\
\hline & $\begin{array}{l}5110 \text { General taxes } \\
5121 \text { Excises }\end{array}$ & $\begin{array}{l}28180 \\
20494\end{array}$ & $\begin{array}{l}35123 \\
21906\end{array}$ & $\begin{array}{l}40086 \\
22809\end{array}$ & $\begin{array}{l}45486 \\
24357\end{array}$ \\
\hline (4) & Taxes on production sale transfer $(5100)$ & 60438 & 69922 & 75994 & 85023 \\
\hline (5) & Taxes on Goods and Services (5000) & 65843 & 76331 & 82880 & 92872 \\
\hline \multicolumn{6}{|c|}{ Implicit average indirect tax rate on consumption out of benefit income: } \\
\hline (6) & using general consumption taxes plus excise duties (3)/(2) & $9.6 \%$ & $9.8 \%$ & $9.5 \%$ & $9.0 \%$ \\
\hline (7) & using a broad concept of the indirect tax base (5)/(2) & $12.9 \%$ & $13.1 \%$ & $12.6 \%$ & $12.0 \%$ \\
\hline (8) & using a broad concept of the indirect tax base and ignoring government consumpion (5)/(1) & $14.8 \%$ & $15.1 \%$ & $14.7 \%$ & $14.2 \%$ \\
\hline
\end{tabular}

Source: OECD on-line National Accounts database (http://stats.oecd.org/Indexaspx?DataSetCode=SNA TABLE1) for lines 1 and 2; and OECD Revenue Statistics database (http://stats.oecd.org/Index.aspx?DataSetCode=REV) for lines 3, 4, and 5. 
DELSA/ELSA/WD/SEM(2011)9

Table Annex I.A.2.1

Detailed information on the impact of the tax system on social expenditure (cont.)

AUSTRALIA

C. Tax breaks for social purposes (in millions of Australian dollars)

\begin{tabular}{|c|c|c|c|c|}
\hline & 2001 & 2003 & 2005 & 2007 \\
\hline Tax breaks similar to cash benefits & 1929 & 2138 & 7951 & 8614 \\
\hline Taxoffsets for taxpayers with dependants & 16 & 15 & 20 & - \\
\hline Tax offset for housekeeper who cares for a prescribed dependant & 360 & 370 & 390 & - \\
\hline Tax offset for low income earners & 460 & 400 & 670 & - \\
\hline Exemption for Medicare level for residents with a taxable income below a threshold & 340 & 380 & 374 & 446 \\
\hline Medical expenses tax offset & 150 & 220 & 305 & 385 \\
\hline Exemption of rent subsidy payments under the Commonwealth/State mortgage and rent relief schemes & 13 & 13 & 3 & 3 \\
\hline $30 \%$ tax offset for expenditure on private health insurance & 590 & 740 & 900 & 1020 \\
\hline Exemption of the Baby Bonus & - & - & 140 & 165 \\
\hline Senior Australian Tax Offset & - & - & 606 & 410 \\
\hline Tax offset for child care & - & - & 0 & 365 \\
\hline Tax offset for dependant spouse, child-housekeeper and housekeeper who cares for prescribed dependent & - & - & - & 319 \\
\hline Tax offsets for taxpayers supporting a parent, parent-in-law, or invalid relative & - & - & 20 & 40 \\
\hline Mature Age Worker Tax Offset & - & - & 425 & 455 \\
\hline Exemption of certain income support benefits, pensions or allowances & - & - & 820 & 1000 \\
\hline Exemption of certain war-related payments and pensions & - & - & 290 & 250 \\
\hline Exemption of Child Care Benefit & - & - & 410 & 445 \\
\hline Exemption of Family Tax Benefit,Parts A and B, including expense equivalent & - & - & 1880 & 2480 \\
\hline Exemption of the first child tax offset (Baby Bonus) & - & - & 31 & 17 \\
\hline Exemption of Utilities Allowance and Seniors' Concession Allowance & - & - & 14 & 38 \\
\hline Exemption of payments made under the First Home Owners Grant Scheme & - & - & 285 & 325 \\
\hline Income averaging for authors, inventors, performing artists, production associates and sportspersons & - & - & 7 & 10 \\
\hline Exemption of post-judgment injury awards in personal injury compensation cases & - & - & 2 & 2 \\
\hline Child Care Services (GST - Goods and Services Tax) & - & - & 360 & 440 \\
\hline Tax breaks to stimulate private social protection (not including pensions) & 580 & 770 & 1092 & 1780 \\
\hline Partial rebate for certain non-profit, non-government bodies & 40 & 20 & 19 & 45 \\
\hline Deduction for gifts to approved donees & 300 & 540 & 730 & 810 \\
\hline Capped exemption for public benevolent institutions (excluding public hospitals) & 240 & 210 & 250 & 710 \\
\hline Deduction for contributions with an associated minor benefit & - & - & 3 & 5 \\
\hline Deduction for donations to prescribed private funds & - & - & 90 & 210 \\
\hline \multicolumn{5}{|l|}{ Memorandum Items } \\
\hline Tax breaks for pensions & 10575 & 14255 & 20285 & 31690 \\
\hline Concessional taxation of funded superannuation & 9215 & 13400 & 17930 & 26600 \\
\hline Concessional taxation of unfunded superannuation lump sums & - & 140 & 150 & 380 \\
\hline Concessional treatment of non-superannuation termination benefits & 990 & 320 & 310 & 1200 \\
\hline Capped taxation rates for lump sumpayments for unused recreation and long service leave & 210 & 190 & 150 & 115 \\
\hline Taxation of five per cent of unused longservice leave accumulated by 15 August 1978 & 135 & 85 & 85 & 75 \\
\hline Capital gains tax exemption on the sale of a small business at retirement & 25 & 120 & 180 & 410 \\
\hline Superannuation - capital gains tax discount for funds & .. & .. & 1090 & 890 \\
\hline Superannuation - deduction and concess ional taxation of certain personal contributions & .. & .. & 410 & 1550 \\
\hline Superannuation - measures for low-income earners & .. & .. & 90 & 550 \\
\hline Superannuation - spouse contribution offset & .. & .. & 15 & 10 \\
\hline Superannuation - tax on funded lump sums relating to post-June 1983 service & .. & .. & -160 & -180 \\
\hline Small business capital gains tax exemption for assets held for more than 15 years & .. & .. & 35 & 90 \\
\hline
\end{tabular}

Source: Australian Government (2009, 2007,2005, 2003), Tax Expenditure Statement, The Treasury, Canberra. 
DELSA/ELSA/WD/SEM(2011)9

Table Annex I.A.2.1

Detailed information on the impact of the tax system on social expenditure (cont.)

AUSTRIA

A. Average Itemised Tax Rates (AITR \% )

\begin{tabular}{|c|c|c|c|c|}
\hline & 2001 & 2003 & 2007 & 2007 \\
\hline Old-age cash benefits $(1+3)$ & $17.7 \%$ & & & \\
\hline - public pensions & & $17.0 \%$ & $16.6 \%$ & $15.9 \%$ \\
\hline - private pensions & & $13.0 \%$ & $16.6 \%$ & $15.9 \%$ \\
\hline \multicolumn{5}{|l|}{ Incapacity-related benefits } \\
\hline - Disability pensions & $17.7 \%$ & $17.0 \%$ & $16.6 \%$ & $15.9 \%$ \\
\hline - Occupational Injury benefits & $0.0 \%$ & $0.0 \%$ & $0.0 \%$ & $0.0 \%$ \\
\hline - Sickness payments & $30.0 \%$ & $30.0 \%$ & $29.3 \%$ & $22.0 \%$ \\
\hline
\end{tabular}

Source: Ministry of Finance (Bundesministerium fur Finanzen), Wage Tax Statistics (2001, 2003, 2005, 2007).

B. Average implicit indirect tax rates of consumption out of benefit income

Indirect taxes paid out of consumption of cash transfers, in millions of euros

\begin{tabular}{|c|c|c|c|c|c|}
\hline & & 2001 & 2003 & 2005 & 2007 \\
\hline (1) & Private final consumption expenditure & 117223 & 122481 & 133767 & 143812 \\
\hline (2) & Private consumption plus Government consumption minus Government wages & 136387 & 143029 & 156019 & 168524 \\
\hline \multirow[t]{2}{*}{ (3) } & General consumption taxes plus excise duties $(5110+5121)$ & 22917 & 24052 & 25935 & 27634 \\
\hline & $\begin{array}{l}5110 \text { General taxes } \\
5121 \text { Excises }\end{array}$ & $\begin{array}{c}17301 \\
5616\end{array}$ & $\begin{array}{c}17944 \\
6108\end{array}$ & $\begin{array}{c}19466 \\
6469\end{array}$ & $\begin{array}{c}20988 \\
6646\end{array}$ \\
\hline (4) & Taxes on production sale transfer $(5100)$ & 24814 & 26043 & 27863 & 29716 \\
\hline (5) & Taxes on Goods and Services (5000) & 26438 & 27813 & 29615 & 31574 \\
\hline \multicolumn{6}{|c|}{ Implicit average indirect tax rate on consumption out of benefit income: } \\
\hline (6) & using general consumption taxes plus excise duties $(3) /(2)$ & $16.8 \%$ & $16.8 \%$ & $16.6 \%$ & $16.4 \%$ \\
\hline "(7) & using a broad concept of the indirect tax base (5)/(2) & $19.4 \%$ & $19.4 \%$ & $19.0 \%$ & $18.7 \%$ \\
\hline (8) & using a broad concept of the indirect tax base and ignoring government consumpion (5)/(1) & $22.6 \%$ & $22.7 \%$ & $22.1 \%$ & $22.0 \%$ \\
\hline
\end{tabular}

Source: OECD on-line National Accounts database (http://stats.oecd.org/Index.aspx?DataSetCode=SNA_TABLE1) for lines 1 and 2; and OECD Revenue Statistics database (http://stats.oecd.org/Index.aspx?DataSetCode=REV) for lines 3, 4, and 5.

\section{Tax breaks for social purposes (in millions of euros)}

\begin{tabular}{|c|c|c|c|c|}
\hline & 2001 & 2003 & 2005 & 2007 \\
\hline Tax breaks similar to cas $h$ benefits & 42.0 & 82.0 & 152.0 & 152.0 \\
\hline Appliances for the disabled (Befreiung für Versehrten- und Invalidenfahrzeuge) $\S 2 \mathrm{Abs} .1 \mathrm{Z} \mathrm{5,12}$ & 2.0 & 2.0 & 2.0 & 2.0 \\
\hline Special tax relief (Außergewöhnliche Belastungen) $\S 34,35$ & 40.0 & 40.0 & 40.0 & 40.0 \\
\hline Tax credits for one-parent families & & 40.0 & 110.0 & 110.0 \\
\hline Tax breaks to stimulate private social protection (not including pensions) & 20.0 & 20.0 & 20.0 & 20.0 \\
\hline Contributions to health, accident and pension insurance (Versicherungs beiträge) $\S 18 \mathrm{Abs} .1 \mathrm{Z} 2$ & 20.0 & 20.0 & 20.0 & 20.0 \\
\hline \multicolumn{5}{|l|}{ Memorandum Items } \\
\hline Tax breaks for pensions & 130.0 & 130.0 & 195.0 & 205.0 \\
\hline - Deduction of contributions to private pension insurances or funds as "special expenses" & 130.0 & 130.0 & 150.0 & 150.0 \\
\hline - Premium (payable tax credit) for contributions to pension funds & & & 45.0 & 55.0 \\
\hline
\end{tabular}

Source: Budget Accounts, Forderungsbericht (2001, 2003, 2005, 2007), Ministry of Finance, Austria. 
DELSA/ELSA/WD/SEM(2011)9

Table Annex I.A.2.1

Detailed information on the impact of the tax system on social expenditure (cont.)

BELGIUM

A. Amount of direct tax paid on benefit income (in millions of euros)

\begin{tabular}{|c|c|c|c|c|}
\hline & 2001 & 2003 & 2005 & 2007 \\
\hline A.Taxes paid on transfer income (A1 minus A2, then adding local tax rate *) & 4093.5 & 4370.1 & 4079.4 & 4058.1 \\
\hline Pensions: PIT & 5060.1 & 5457.8 & 5972.0 & 3451.1 \\
\hline Sickness: PIT & 449.0 & 495.7 & 485.0 & 178.0 \\
\hline Early retirement: PIT & 2.1 & 0.8 & & \\
\hline Unemployment benefits: PIT & 525.2 & 581.1 & 536.0 & 149.4 \\
\hline Total PIT (A1) & 6036.4 & 6535.4 & 6993.0 & 3778.5 \\
\hline - Pensions: tax credit & 1722.1 & 1923.9 & 2525.0 & - \\
\hline - Sickness: tax credit & 177.2 & 213.3 & 304.0 & - \\
\hline - Early retirement: tax credit & 1.2 & 0.3 & - & - \\
\hline - Unemployment benefits: tax credit & 214.5 & 301.5 & 323.0 & - \\
\hline - Others & 98.4 & 26.4 & 32.0 & - \\
\hline Total tax credits (A2) & 2213.4 & 2465.4 & 3184.0 & \\
\hline Local tax rate & $7.1 \%$ & $7.4 \%$ & $7.1 \%$ & $7.4 \%$ \\
\hline B. Social security contributions & 885.1 & 999.3 & 1109.4 & 1182.0 \\
\hline Total $(A+B)$ & 4978.6 & 5369.4 & 5188.8 & 5240.1 \\
\hline
\end{tabular}

* Local tax rate is applied to income tax, which overestimates local tax amount as local taxes are only paid when the amount of the credit does not exhaust income tax.

B. Average implicit indirect tax rates of consumption out of benefit income

Indirect taxes paid out of consumption of cash transfers, in millions of euros

\begin{tabular}{|c|c|c|c|c|c|}
\hline & & 2001 & 2003 & 2005 & 2007 \\
\hline (1) & Private final consumption expenditure & 138912 & 144383 & 155751 & 170965 \\
\hline (2) & Private consumption plus Government consumption minus Government wages & 164980 & 173729 & 188353 & 206442 \\
\hline \multirow[t]{3}{*}{ (3) } & General consumption taxes plus excise duties $(5110+5121)$ & 23692 & 25284 & 28981 & 31191 \\
\hline & 5110 General taxes & 18060 & 19039 & 21854 & 23931 \\
\hline & 5121 Excises & 5632 & 6245 & 7126 & 7259 \\
\hline (4) & Taxes on production sale transfer $(5100)$ & 26052 & 27952 & 31772 & 34506 \\
\hline (5) & Taxes on Goods and Services (5000) & 28374 & 30165 & 34152 & 36957 \\
\hline \multicolumn{6}{|c|}{ Implicit average indirect tax rate on consumption out of benefit income: } \\
\hline (6) & using general consumption taxes plus excise duties $(3) /(2)$ & $14.4 \%$ & $14.6 \%$ & $15.4 \%$ & $15.1 \%$ \\
\hline (7) & using a broad concept of the indirect tax base (5)/(2) & $17.2 \%$ & $17.4 \%$ & $18.1 \%$ & $17.9 \%$ \\
\hline (8) & using a broad concept of the indirect tax base and ignoring government consumpion (5)/(1) & $20.4 \%$ & $20.9 \%$ & $21.9 \%$ & $21.6 \%$ \\
\hline
\end{tabular}

Source: OECD on-line National Accounts database (http://stats.oecd.org/Indexaspx?DataSetCode=SNA_TABLE1) for lines 1 and 2; and OECD Revenue Statistics database (http://stats.oecd.org/Indexaspx?DataSetCode=REV) for lines 3,4 , and 5 .

C. Tax breaks for social purposes (in million of Euros)

\begin{tabular}{|c|c|c|c|c|}
\hline & 2001 & 2003 & 2005 & 2007 \\
\hline Tax breaks similar to cash benefits & 1355.8 & 1521.0 & 1827.0 & 1968.1 \\
\hline Tax credit for children & 1275.6 & 1443.8 & 1722.9 & 1861.4 \\
\hline Compl. Sickness contr. & 0.0 & 0.0 & 0.0 & 0.0 \\
\hline Allowance "ALE" & 16.9 & 17.3 & 12.1 & 13.1 \\
\hline Allowance Childcare expenses & 63.3 & 59.9 & 92.1 & 93.6 \\
\hline Tax breaks to stimulate private social protection (not including pensions) & 0.0 & 0.0 & 0.0 & 0.0 \\
\hline \multicolumn{5}{|l|}{ Memorandum Items } \\
\hline Tax breaks for pensions & 363.3 & 382.1 & 518.5 & 475.5 \\
\hline Pension savings (3rd pillar) & 262.8 & 287.7 & 409.7 & 387.9 \\
\hline Pension savings ( $2 \mathrm{~d}$ pillar) & 100.5 & 94.4 & 108.8 & 87.6 \\
\hline
\end{tabular}

Source: Ministry of Finance, Belgium, from IPP (Impôt des Personnes Physiques). 
DELSA/ELSA/WD/SEM(2011)9

Table Annex I.A.2.1

Detailed information on the impact of the tax system on social expenditure (cont.)

CANADA

A. Average Itemised Tax Rates (AITR \%)

\begin{tabular}{|c|c|c|c|c|}
\hline & 2001 & 2003 & 2005 & 2007 \\
\hline 1 Old-age Cash Benefits & $15.02 \%$ & $14.28 \%$ & $14.61 \%$ & $13.78 \%$ \\
\hline la - Public Pensions (OAS) & $6.06 \%$ & $5.76 \%$ & $5.89 \%$ & $5.91 \%$ \\
\hline $\mathrm{lb}$ - Private Pensions & $18.49 \%$ & $17.54 \%$ & $17.70 \%$ & $16.47 \%$ \\
\hline 2 Canadian Pension Plan (Retirement) & $9.46 \%$ & $8.70 \%$ & $8.78 \%$ & $8.72 \%$ \\
\hline 3 Canadian Pension Plan (Disability) & $2.57 \%$ & $1.86 \%$ & $1.94 \%$ & $1.74 \%$ \\
\hline 4 Employment Insurance & $9.05 \%$ & $8.35 \%$ & $6.54 \%$ & $6.01 \%$ \\
\hline 4a - - Regular Employment Insurance & .. & $8.63 \%$ & $7.07 \%$ & $6.21 \%$ \\
\hline 4b - Parental Leave Payments & .. & .. & .. & .. \\
\hline 4c - Active Labour Market Programs & .. & $0.37 \%$ & $1.41 \%$ & $-0.58 \%$ \\
\hline 5 Worker's Compensation & $2.41 \%$ & $2.48 \%$ & $1.41 \%$ & $1.46 \%$ \\
\hline 6 Guaranteed Income Supplement & $0.52 \%$ & $0.54 \%$ & $1.01 \%$ & $1.17 \%$ \\
\hline 7 Social Assistance & $0.02 \%$ & $0.12 \%$ & $0.14 \%$ & $-0.21 \%$ \\
\hline 8 Wage Income & $22.20 \%$ & $22.13 \%$ & $21.48 \%$ & $21.12 \%$ \\
\hline
\end{tabular}

The sample used for the microdataset simulation was a stratified sample of approximately 450000 records, weighted to represent all taxfilers in Canada. The sample is provided by the Canada Revenue Agency.

B. Average implicit indirect tax rates of consumption out of benefit income

Indirect taxes paid out of consumption of cash transfers, in millions of Canadian dollars

\begin{tabular}{|c|c|c|c|c|c|}
\hline & & 2001 & 2003 & 2005 & 2007 \\
\hline (1) & Private final consumption expenditure & 608549 & 670618 & 760701 & 851603 \\
\hline "(2) & Private consumption plus Government consumption minus Government wages & 693669 & 763841 & 866787 & 969399 \\
\hline \multirow[t]{2}{*}{ "(3) } & General consumption taxes plus excise duties $(5110+5121)$ & 77609 & 85489 & 92865 & 93475 \\
\hline & $\begin{array}{l}5110 \text { General taxes } \\
5121 \text { Excises }\end{array}$ & $\begin{array}{l}58093 \\
19516\end{array}$ & $\begin{array}{l}62604 \\
22885\end{array}$ & $\begin{array}{l}69902 \\
22963\end{array}$ & $\begin{array}{l}70385 \\
23090\end{array}$ \\
\hline "(4) & Taxes on production sale transfer $(5100)$ & 92940 & 102262 & 111536 & 113753 \\
\hline (5) & Taxes on Goods and Services (5000) & 98466 & 108132 & 118599 & 121925 \\
\hline \multicolumn{6}{|c|}{ Implicit average indirect tax rate on consumption out of benefit income: } \\
\hline (6) & using general consumption taxes plus excise duties (3)/(2) & $11.2 \%$ & $11.2 \%$ & $10.7 \%$ & $9.6 \%$ \\
\hline (7) & using a broad concept of the indirect tax base (5)/(2) & $14.2 \%$ & $14.2 \%$ & $13.7 \%$ & $12.6 \%$ \\
\hline "(8) & using a broad concept of the indirect tax base and ignoring government consumpion (5)/(1) & $16.2 \%$ & $16.1 \%$ & $15.6 \%$ & $14.3 \%$ \\
\hline
\end{tabular}

Source: OECD on-line National Accounts database (http://stats.oecd.org/Indexaspx?DataSetCode=SNA_TABLEl) for lines 1 and 2; and OECD Revenue Statistics database (http://stats.oecd.org/Indexaspx?DataSetCode=REV) for lines 3,4 , and 5 . 
DELSA/ELSA/WD/SEM(2011)9

Table Annex I.A.2.1

Detailed information on the impact of the tax system on social expenditure (cont.)

CANADA

C. Tax breaks for social purposes (in millions of Canadian dollars)

\begin{tabular}{|c|c|c|c|c|}
\hline \multirow[b]{2}{*}{ Tax breaks similar to cash benefits (total without 5 and 8 below) } & \multirow{2}{*}{$\begin{array}{c}2001 \\
\mathbf{1 1 1 8 9}\end{array}$} & \multirow{2}{*}{$\begin{array}{c}2003 \\
12741\end{array}$} & \multirow{2}{*}{$\begin{array}{c}2005 \\
13914\end{array}$} & \multirow{2}{*}{$\begin{array}{r}2007 \\
20519\end{array}$} \\
\hline & & & & \\
\hline 1 Disability TaxCredit & 464 & 512 & 561 & 685 \\
\hline 2 Provincial Tax Reduction & 339 & 409 & 530 & 615 \\
\hline 3 Infirm dependant credit & & & & \\
\hline Caregiver credit & 85 & 107 & 117 & 126 \\
\hline 4 Attendant Care expense & 0.3 & 0.8 & 1.7 & 3.2 \\
\hline 5 Canadian Child Tax Benefit/National Child Benefit (amount not included in total TBSP, as already accounted in SOCX & 7640 & 8185 & 9145 & 9633 \\
\hline 6 Medical expense supplement for earners & 55 & 68 & 92 & 111 \\
\hline 7 Deductibility of charitable donations from corporate income tax & 260 & 290 & 345 & 465 \\
\hline 8 Age amount (amount not included in total TBSP, as already taken into account when calculating AITRs) & 1916 & 2036 & 2010 & 2496 \\
\hline 9 Medical expense tax credit & 920 & 1116 & 1476 & 1349 \\
\hline 10 Child care expense deduction & 1112 & 1002 & 1207 & 1372 \\
\hline 11 Adoption Expense Deduction & - & - & 4 & 4 \\
\hline 12 Amount for an eligible dependant & 824 & 873 & 880 & 984 \\
\hline 13 Non-taxation of employer paid premiums & 4444 & 5474 & 5989 & 5751 \\
\hline 14 Portion of refundable tax credits that offsets tax liability & 2686 & 2890 & 2712 & 2314 \\
\hline 15 Non-taxation of employee-paid EI premiums & - & - & - & 2801 \\
\hline 16 Employment credit & - & - & - & 1838 \\
\hline 17 Universal child care benefit (amount not included in total TBSP) & - & - & - & 2714 \\
\hline 18 Children's fitness tax credit & - & - & - & 87 \\
\hline 19 Working income tax benefit & - & - & - & 602 \\
\hline 20 Child tax credit & - & - & - & 1411 \\
\hline 21 Investment tax credit for child care spaces & - & - & - & 2 \\
\hline Tax breaks to stimulate private social protection (not including pensions) & 7646 & 9309 & 13116 & 13427 \\
\hline Non-Taxation of employer-paid CPP premiums & 4445 & 5474 & 5989 & 5751 \\
\hline Non-Taxation of employer-paid El premiums & - & & 2735 & 2600 \\
\hline Non-taxation of employer paid health and dental benefits & 1710 & 2010 & 2135 & 2490 \\
\hline Charitable donations credit & 1491 & 1825 & 2257 & 2537 \\
\hline Non-taxation of donations of publicly-listed securities & - & - & - & 50 \\
\hline \multicolumn{5}{|l|}{ Memorandum item } \\
\hline Tax breaks for pensions $(1+2+3)$ & 5611 & 14628 & 24144 & 30985 \\
\hline \multicolumn{5}{|l|}{ Revenue foregone method: } \\
\hline 1 Pension Income Deduction & 626 & 653 & 679 & 1315 \\
\hline \multicolumn{5}{|l|}{2 Registered retirement savings plans (RRSPs) } \\
\hline Deduction for contributions & 6225 & 6000 & 6760 & 7585 \\
\hline Non-taxation of investment income & 1280 & 4080 & 7160 & 9090 \\
\hline Taxation of withdrawals & -3465 & -3670 & -4155 & -4600 \\
\hline Net tax expenditure & 4040 & 6410 & 9765 & 12075 \\
\hline \multicolumn{5}{|l|}{3 Registered pension plans (RPPs) } \\
\hline Deduction for contributions & 4575 & 6615 & 8415 & 9450 \\
\hline Non-taxation of investment income & 2785 & 7530 & 12465 & 14875 \\
\hline Taxation of withdrawals & -6415 & -6580 & -7180 & -6730 \\
\hline Net tax expenditure & 945 & 7565 & 13700 & 17595 \\
\hline \multicolumn{5}{|l|}{ Supplementary information: } \\
\hline Present value of taxassistance for retirement savings plans * & 5670 & 6820 & 8340 & 9395 \\
\hline
\end{tabular}

Data supplied by Finance Canada, from Department of Finance Canada (2009, 2004, 2002), Tax Expenditures and Evaluations, Ottawa. 
DELSA/ELSA/WD/SEM(2011)9

Table Annex I.A.2.1

Detailed information on the impact of the tax system on social expenditure (cont.)

\section{CZECH REPUBLIC}

A. Amount of direct tax paid on benefit income (in millions of Czech koruny)

\begin{tabular}{|c|c|c|c|c|}
\hline & 2001 & 2003 & 2005 & 2007 \\
\hline Total tax paid on transfer income & & & & \\
\hline old-age pensions & 1.0 & 3.0 & 14.6 & 11.0 \\
\hline
\end{tabular}

Source: Ministry of Finance, Tax Policy Unit; Czech Social Security Administation.

B. Average implicit indirect tax rates of consumption out of benefit income

Indirect taxes paid out of consumption of cash transfers, in millions of Czech koruny

\begin{tabular}{|c|c|c|c|c|c|}
\hline & & 2001 & 2003 & 2005 & 2007 \\
\hline (1) & Private final consumption expenditure & 1220316 & 1332470 & 1464486 & 1686837 \\
\hline (2) & Private consumption plus Government consumption minus Government wages & 1544042 & 1721471 & 1885005 & 2136460 \\
\hline \multirow[t]{2}{*}{ (3) } & General consumption taxes plus excise duties $(5110+5121)$ & 226686 & 251925 & 325652 & 365780 \\
\hline & $\begin{array}{l}5110 \text { General taxes } \\
5121 \text { Excises }\end{array}$ & $\begin{array}{c}149893 \\
76793\end{array}$ & $\begin{array}{c}164250 \\
87675\end{array}$ & $\begin{array}{l}215118 \\
110534\end{array}$ & $\begin{array}{l}232288 \\
133492\end{array}$ \\
\hline (4) & Taxes on production sale transfer $(5100)$ & 236864 & 262453 & 325861 & 365998 \\
\hline (5) & Taxes on Goods and Services (5000) & 255029 & 285108 & 350949 & 393042 \\
\hline \multicolumn{6}{|c|}{ Implicit average indirect tax rate on consumption out of benefit income: } \\
\hline (6) & using general consumption taxes plus excise duties (3)/(2) & $14.7 \%$ & $14.6 \%$ & $17.3 \%$ & $17.1 \%$ \\
\hline (7) & using a broad concept of the indirect tax base (5)/(2) & $16.5 \%$ & $16.6 \%$ & $18.6 \%$ & $18.4 \%$ \\
\hline (8) & using a broad concept of the indirect tax base and ignoring government consumpion (5)/(1) & $20.9 \%$ & $21.4 \%$ & $24.0 \%$ & $23.3 \%$ \\
\hline
\end{tabular}

Source: OECD on-line National Accounts database (http://stats.oecd.org/Index.aspx?DataSetCode=SNA TABLE1) for lines 1 and 2; and OECD Revenue Statistics database (http://stats.oecd.org/Index.aspx?DataSetCode=REV) for lines 3, 4, and 5 . 
DELSA/ELSA/WD/SEM(2011)9

Table Annex I.A.2.1

Detailed information on the impact of the tax system on social expenditure (cont.)

\section{CZECH REPUBLIC}

C. Tax breaks for social purposes (in millions of Czech koruny)

\begin{tabular}{|c|c|c|c|c|}
\hline & 2001 & 2003 & 2005 & 2007 \\
\hline Tax breaks similar to cash benefits & 11084 & 11731 & 14688 & 18532 \\
\hline \multicolumn{5}{|l|}{ 1. Tax breaks similar to cash benefits } \\
\hline \multicolumn{5}{|l|}{ 1.1 Tax exemptions (Personal Income Tax): } \\
\hline $\begin{array}{l}\text { * non-monetary benefits covered from the fund for cultural and social needs or profit after tax provided by an } \\
\text { employer to his employee in form of recreational, health care, educational facilities, etc. }\end{array}$ & 528 & 648 & 795.3 & 976.0 \\
\hline \multicolumn{5}{|l|}{ 1.2 Allowances from the tax base (Personal Income Tax; social insurance contributions are deductible from the tax base): } \\
\hline * per each dependent child living with the taxpayer in one household & 10000 & 10500 & \multirow{2}{*}{13484} & \multirow{2}{*}{16683} \\
\hline * per each handicapped dependent child requiring an escort & 188 & 197 & & \\
\hline $\begin{array}{l}\text { * per handicapped spouse requiring an escort living with taxpayer in one household unless the spouse's own } \\
\text { income exceeds low income limit }\end{array}$ & 8 & 8 & 9 & 2 \\
\hline * per handicapped taxpayer requiring an escort & 60 & 63 & 66 & 34 \\
\hline $\begin{array}{l}\text { * gifts donated to municipalities or to legal entities for financing science, education, culture, schools, police, } \\
\text { youth welfare, animal protection, environment, humanitarian projects etc. }\end{array}$ & 300 & 315 & 334 & 837 \\
\hline Tax breaks to stimulate private social protection (not including pensions) & 4073 & 1485 & 1701 & 1966 \\
\hline \multicolumn{5}{|l|}{2.1 Corporate Income Tax } \\
\hline * tax credits for disabled employees & 3973 & 1352 & 1564 & 1801 \\
\hline \multicolumn{5}{|l|}{ 2.2 Personal Income Tax } \\
\hline * tax credits for disabled employee & 100 & 133 & 137 & 165 \\
\hline \multicolumn{5}{|l|}{ Memorandum item } \\
\hline Tax breaks for pensions & 1105 & 1679 & 3390 & 3724 \\
\hline 1. Deduction of contributions to private pensions - income tax exemptions and allowances from the tax base & & & & \\
\hline $\begin{array}{l}\text { * exemption of contributions of employers on behalf of their employees on pension insurance with state } \\
\text { contribution from personal income tax up to a ceiling of } 5 \text { per cent of employer's gross wage }\end{array}$ & 380 & 540 & 779 & 850 \\
\hline $\begin{array}{l}\text { * deduction of contributions of employers on behalf of their employees on pension insurance with state } \\
\text { contribution from employer's tax base up to a ceiling of } 3 \text { per cent of gross wage of the employee }\end{array}$ & 465 & 759 & 694 & 612 \\
\hline * contributions of employees on their pension insurance with state contribution & 260 & 380 & 644 & 814 \\
\hline * deduction on behalf on pension insurance with state contribution from personal income tax up to a ceiling & - & - & 1273 & 1448 \\
\hline \multicolumn{5}{|l|}{ 2. Non-taxation of investment of private pension funds } \\
\hline * there is income tax of $15 \%$ from the returns of private pension funds - standard tax rate is $28 \%$ & - & - & - & - \\
\hline * reduced $15 \%$ withholding tax on benefits/returns paid by the pension funds to the contributors & .. & .. & .. & - \\
\hline * reduced $15 \%$ withholding tax on benefits/returns paid by the life insurance to the contributors & .. & .. &.. & - \\
\hline
\end{tabular}

Sources: Tax Statistics, Czech Ministry of Finance; and the Association of Pension Funds of the Czech Republic. 
DELSA/ELSA/WD/SEM(2011)9

Table Annex I.A.2.1

Detailed information on the impact of the tax system on social expenditure (cont.)

DENMARK

A. Average Itemis ed Tax Rates - AITR \%

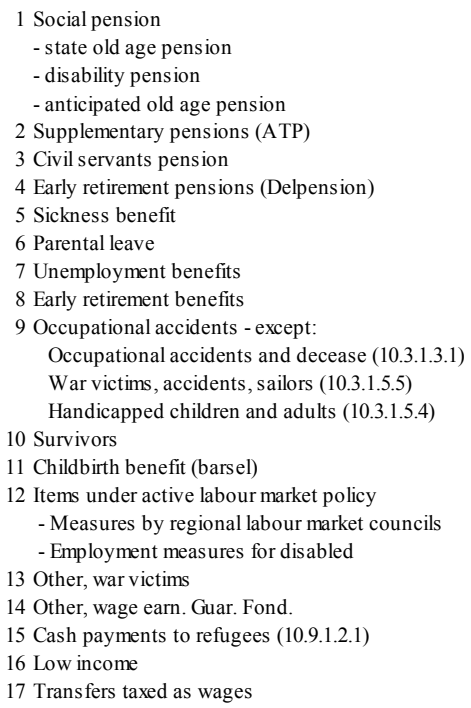

Taxand Social security Taxand Social securitxax an $\frac{2005}{\underline{d \text { Social securitjax and Social secu1 }}}$

$\begin{array}{lllllll}27.68 & (98 \%) & 27.20 & (98 \%) & 27.25 & (98 \%) & 27.13 \\ 26.92 & (75 \%) & 26.22 & (75 \%) & 26.38 & (77 \%) & 27.16 \\ 23.78 & (84 \%) & 23.69 & (84 \%) & 26.57 & (90 \%) & 23.37 \\ 30.68 & & 29.79 & & 29.83 & & 29.56 \\ 34.77 & & 34.00 & & 33.66 & & 33.81 \\ 32.30 & & 31.48 & & 30.19 & & 30.00 \\ 36.36 & & 36.49 & & 36.75 & & 35.16 \\ 28.85 & & 28.56 & & 28.69 & & 29.47 \\ 32.78 & & 32.66 & & 32.58 & & 30.80 \\ 29.38 & & 28.16 & & 28.09 & & 30.29 \\ 36.07 & & 32.05 & & 36.29 & & 32.70 \\ & & & & 35.34 & (22 \%) & 35.09 \\ & & & & & (60 \%) & 32.70 \\ 10.00 & & 10.00 & & 10.00 & & 39.85 \\ 39.71 & & 37.21 & & 37.59 & & 35.44 \\ & & & & & & \\ 26.89 & & 26.42 & & 26.02 & & 25.37 \\ 31.30 & & 30.72 & & 30.87 & & 30.21 \\ 35.30 & (90 \%) & 33.95 & (90 \%) & 33.50 & (84 \%) & 33.46 \\ 44.10 & & 39.31 & & 35.40 & & 37.48 \\ & & & & 5.70 & & 3.18 \\ 26.73 & & 26.04 & & 25.80 & & 24.97 \\ 43.54 & & 42.07 & & 41.21 & & 39.85 \\ & & & & & \end{array}$

In parentheses: proportion of spending subject to taxation if not $100 \%$.

B. Average implicit indirect tax rates of cons umption out of benefit income

Indirect taxes paid out of consumption of cash transfers, in millions Danish kroner

\begin{tabular}{|c|c|c|c|c|c|}
\hline & & 2001 & 2003 & 2005 & 2007 \\
\hline (1) & Private final consumption expenditure & 631687 & 666942 & 745139 & 821664 \\
\hline (2) & Private consumption plus Government consumption minus Government wages & 742089 & 785707 & 880249 & 976555 \\
\hline \multirow[t]{2}{*}{ (3) } & General consumption taxes plus excise duties $(5110+5121)$ & 195178 & 203394 & 230549 & 254214 \\
\hline & $\begin{array}{l}5110 \text { General taxes } \\
5121 \text { Excises }\end{array}$ & $\begin{array}{l}127938 \\
67240\end{array}$ & $\begin{array}{c}134442 \\
68952\end{array}$ & $\begin{array}{c}154698 \\
75851\end{array}$ & $\begin{array}{c}175426 \\
78788\end{array}$ \\
\hline (4) & Taxes on production sale transfer $(5100)$ & 199772 & 208841 & 237655 & 260896 \\
\hline (5) & Taxes on Goods and Services $(5000)$ & 211685 & 220960 & 251007 & 275763 \\
\hline \multicolumn{6}{|c|}{ Implicit average indirect tax rate on consumption out of benefit income: } \\
\hline (6) & using general cons umption taxes plus excise duties $(3) /(2)$ & $26.3 \%$ & $25.9 \%$ & $26.2 \%$ & $26.0 \%$ \\
\hline (7) & using a broad concept of the indirect tax base (5)/(2) & $28.5 \%$ & $28.1 \%$ & $28.5 \%$ & $28.2 \%$ \\
\hline (8) & using a broad concept of the indirect tax base and ignoring government consumpion (5)/(1) & $33.5 \%$ & $33.1 \%$ & $33.7 \%$ & $33.6 \%$ \\
\hline
\end{tabular}

Source: OECD on-line National Accounts database (http://stats.oecd.org/Indexaspx?DataSetCode=SNA_TABLE1) for lines 1 and 2; and OECD Revenue Statistics database (http://stats.oecd.org/Index.aspx?DataSetCode=REV) for lines 3,4, and 5 .

C. Tax breaks for social purposes (in millions of Danish kroner)

\begin{tabular}{|c|c|c|c|c|}
\hline & 2001 & 2003 & 2005 & 2007 \\
\hline Tax breaks similar to cash benefits & 182 & 189 & 201 & \\
\hline Supplement for older people "Engangsbelob" (67+) & 135 & 140 & 147 & .. \\
\hline Capital tax reduction for older people $(67+)$ & 0 & 0 & & \\
\hline Housing for older people "Plejehjem" & 47 & 49 & 54 & .. \\
\hline Tax breaks to stimulate private social protection (not including pensions) & 0 & 0 & 0 & 0 \\
\hline \multicolumn{5}{|l|}{ Memorandum item } \\
\hline Tax breaks for pensions & 0 & 0 & 0 & 0 \\
\hline
\end{tabular}

Source: Ministry of Finance, Denmark. 
DELSA/ELSA/WD/SEM(2011)9

Table Annex I.A.2.1

Detailed information on the impact of the tax system on social expenditure (cont.)

\section{FINLAND}

A. Average Itemised Tax Rates (\%)

\begin{tabular}{|c|c|c|c|c|c|}
\hline & & 2001 & 2003 & 2005 & 2007 \\
\hline 1 & Old-age cash benefits & & & & \\
\hline 1a & - public pensions & 19.7 & 19.4 & 19.9 & 20.0 \\
\hline $1 \mathrm{c}$ & - private pensions & 28.6 & 28.9 & 28.7 & 28.4 \\
\hline 2 & Survivors' benefits & & & & \\
\hline $2 \mathrm{a}$ & - public pensions & 19.1 & 19.8 & 19.9 & 18.6 \\
\hline 3 & Incapacity-related benefits & & & & \\
\hline $3 a$ & - Disability pensions & 17.5 & 17.6 & 16.5 & 15.5 \\
\hline $3 b$ & - Occupational Injury benefits & 26.2 & 23.5 & 24.3 & 23.0 \\
\hline $3 \mathrm{c}$ & - Sickness payments & 26.8 & 24.4 & 24.5 & 22.4 \\
\hline 4 & Family cash benefits & & & & \\
\hline $4 a$ & - Family benefits & 16.7 & 17.0 & 17.2 & 15.5 \\
\hline $4 \mathrm{~b}$ & - Maternity and parental leave payments & 21.7 & 21.5 & 21.6 & 19.7 \\
\hline 5 & Active labour market policies & & & & \\
\hline $5 \mathrm{a}$ & - benefits while on training & 19.4 & 19.9 & 20.0 & 18.5 \\
\hline 6 & Unemployment & & & & \\
\hline $6 a$ & - unemployment insurance benefit & 20.5 & 20.5 & 20.5 & 18.9 \\
\hline $6 \mathrm{~b}$ & - unemployment assistance benefit & 18.6 & 18.1 & 18.2 & 17.8 \\
\hline
\end{tabular}

The micro-simulation model used in the Ministry of Finance is based on a representative sample of some 25000 individual taxpayers. The model is used for the planning of national tax policies and for estimating the effect of tax policy alterations on tax revenues and on the income tax liabilities of taxpayers at different income levels. The information is in principle collected for the Income Distribution Survey from Statistics Finland. The sample covers about 0,5\% of the total taxpayer population, but the model has been made representative for the total taxpayer population. The dataset is updated annually.

Source: Ministry of Finance.

B. Average implicit indirect tax rates of consumption out of benefit income

Indirect taxes paid out of consumption of cash transfers, in millions of euros

\begin{tabular}{|c|c|c|c|c|c|}
\hline & & 2001 & 2003 & 2005 & 2007 \\
\hline (1) & Private final consumption expenditure & 68877 & 75156 & 81095 & 90708 \\
\hline$(2)$ & Private consumption plus Government consumption minus Government wages & 79559 & 87317 & 94800 & 106090 \\
\hline (3) & General consumption taxes plus excise duties $(5110+5121)$ & 16789 & 18657 & 19623 & 21070 \\
\hline & $\begin{array}{l}5110 \text { General taxes } \\
5121 \text { Excises }\end{array}$ & $\begin{array}{c}11118 \\
5671\end{array}$ & $\begin{array}{l}12455 \\
6202\end{array}$ & $\begin{array}{c}13658 \\
5965\end{array}$ & $\begin{array}{c}15054 \\
6016\end{array}$ \\
\hline (4) & Taxes on production sale transfer $(5100)$ & 18004 & 20011 & 21098 & 22605 \\
\hline (5) & Taxes on Goods and Services (5000) & 18462 & 20509 & 21663 & 23242 \\
\hline \multicolumn{6}{|c|}{ Implicit average indirect tax rate on consumption out of benefit income: } \\
\hline$(6)$ & using general consumption taxes plus excise duties $(3) /(2)$ & $21.1 \%$ & $21.4 \%$ & $20.7 \%$ & $19.9 \%$ \\
\hline (7) & using a broad concept of the indirect tax base $(5) /(2)$ & $23.2 \%$ & $23.5 \%$ & $22.9 \%$ & $21.9 \%$ \\
\hline (8) & using a broad concept of the indirect tax base and ignoring government consumpion (5)/(1) & $26.8 \%$ & $27.3 \%$ & $26.7 \%$ & $25.6 \%$ \\
\hline
\end{tabular}

Source: OECD on-line National Accounts database (http://stats.oecd.org/Index.aspx?DataSetCode=SNA_TABLE1) for lines 1 and 2; and OECD Revenue Statistics database (http://stats.oecd.org/Index.aspx? DataSetCode=REV) for lines 3, 4, and 5.

C. Tax breaks for social purposes (in millions of euros)

\begin{tabular}{|c|c|c|c|c|}
\hline & 2001 & 2003 & 2005 & 2007 \\
\hline Tax breaks similar to cash benefits & $\mathbf{0}$ & $\mathbf{0}$ & $\mathbf{0}$ & $\mathbf{0}$ \\
\hline Tax breaks to stimulate private social protection (not including pensions) & $\mathbf{0}$ & $\mathbf{0}$ & $\mathbf{0}$ & $\mathbf{0}$ \\
\hline \multicolumn{5}{|l|}{ Cash donations to Charities } \\
\hline \multicolumn{5}{|l|}{ Memorandum Items } \\
\hline Tax breaks for pension & 170 & 205 & 220 & 125 \\
\hline - Deduction of contributions to private pensions & 170 & 205 & 220 & 125 \\
\hline
\end{tabular}

Source: Ministry of Finance, Finland. 
DELSA/ELSA/WD/SEM(2011)9

Table Annex I.A.2.1

Detailed information on the impact of the tax system on social expenditure (cont.)

FRANCE

A. Amount of direct tax paid on benefit income (in millions of euros)

\begin{tabular}{|c|c|c|c|c|c|}
\hline & & 2001 & 2003 & 2005 & 2007 \\
\hline & Total (income tax + social security contributions) & 21192.2 & 22247.8 & 25385 & 26549 \\
\hline $1-2-3 a$ & Old-age, survivors' benefits, disability pensions & 15160 & 16292 & 19026 & 19812 \\
\hline $3 b$ & Occupational Injury benefits & 130 & 154 & 156 & 210 \\
\hline $3 \mathrm{c}$ & Sickness payments & 1352 & 1365 & 1675 & 1896 \\
\hline $4 a-4 c$ & Family and sole parent cash benefits & 660 & 684 & 738 & 888 \\
\hline $4 \mathrm{~b}$ & Maternity and parental leave payments & 220 & 283 & 282 & 304 \\
\hline 5 & Active labour market policies & 1570 & 1417 & 973 & 1096 \\
\hline 6 & Unemployment & 2040 & 1987 & 2469 & 2275 \\
\hline 7 & Housing & 60 & 66 & 66 & 68 \\
\hline
\end{tabular}

Sources: See Table C below.

B. Average implicit indirect tax rates of cons umption out of benefit income

Indirect taxes paid out of consumption of cash transfers, in millions of euros

\begin{tabular}{|c|c|c|c|c|c|}
\hline & & 2001 & 2003 & 2005 & 2007 \\
\hline$(1)$ & Private final consumption expenditure & 838227 & 900035 & 981500 & 1074170 \\
\hline$(2)$ & Private consumption plus Government consumption minus Government wages & 980235 & 1062806 & 1161901 & 1268834 \\
\hline \multirow[t]{2}{*}{$(3)$} & General consumption taxes plus excise duties $(5110+5121)$ & 149446 & 158770 & 173531 & 183323 \\
\hline & $\begin{array}{l}5110 \text { General taxes } \\
5121 \text { Excises }\end{array}$ & $\begin{array}{c}109397 \\
40050\end{array}$ & $\begin{array}{c}115770 \\
43000\end{array}$ & $\begin{array}{c}130226 \\
43306\end{array}$ & $\begin{array}{c}139884 \\
43438\end{array}$ \\
\hline$(4)$ & Taxes on production sale transfer $(5100)$ & 161496 & 171585 & 187777 & 197714 \\
\hline$(5)$ & Taxes on Goods and Services (5000) & 165803 & 175788 & 192326 & 203104 \\
\hline \multicolumn{6}{|c|}{ Implicit average indirect tax rate on consumption out of benefit income: } \\
\hline (6) & using general cons umption taxes plus excise duties (3)/(2) & $15.2 \%$ & $14.9 \%$ & $14.9 \%$ & $14.4 \%$ \\
\hline (7) & using a broad concept of the indirect tax base $(5) /(2)$ & $16.9 \%$ & $16.5 \%$ & $16.6 \%$ & $16.0 \%$ \\
\hline$(8)$ & using a broad concept of the indirect tax base and ignoring government consumpion (5)/(1) & $19.8 \%$ & $19.5 \%$ & $19.6 \%$ & $18.9 \%$ \\
\hline
\end{tabular}

Source: OECD on-line National Accounts database (http://stats.oecd.org/Index.aspx?DataSetCode=SNA_TABLE1) for lines 1 and 2; and OECD Revenue Statistics database (http://stats.oecd.org/Index.aspx?DataSetCode=REV) for lines 3, 4, and 5. 
DELSA/ELSA/WD/SEM(2011)9

Table Annex I.A.2.1

Detailed information on the impact of the tax system on social expenditure (cont.)

FRANCE

C. Tax breaks for social purposes (in millions of euros)

\begin{tabular}{|c|c|c|c|c|}
\hline & 2001 & 2003 & 2005 & 2007 \\
\hline Tax breaks Similar to cash benefits & 14750 & 16280 & 18404 & 20153 \\
\hline \multicolumn{5}{|l|}{ Vieillesse } \\
\hline Foncier bâti : Dégrèvement partiel & 50 & 138 & 55 & 82 \\
\hline \multicolumn{5}{|l|}{ Vieillesse - invalidité } \\
\hline Foncier bâti : exonération totale & 290 & 300 & 339 & 506 \\
\hline \multicolumn{5}{|l|}{ Vieillesse - invalidité-survie } \\
\hline Taxe d'habitation : exonération totale & 1220 & 1250 & 1345 & 1327 \\
\hline & & & & 8 \\
\hline Impôt sur le revenu: Réduction pour dépenses d'accueil dans un établissement pour pe & âgées de & tes (long & & 15 \\
\hline \multicolumn{4}{|c|}{ Impôt sur le revenu: Déduction pour cotisations épargne retraite } & 360 \\
\hline \multicolumn{5}{|l|}{ Famille-invalidité } \\
\hline Impôt sur le revenu : quotient familial & 9700 & 9866 & 10987 & 11528 \\
\hline Taxe d'habitation : abattement pour charge de famille & 1070 & 1130 & 1300 & 1283 \\
\hline Taxe d'habitation : effet du quotient familial sur les dégrèvements partiels & 100 & 100 & 120 & 118 \\
\hline \multicolumn{5}{|l|}{ Famille } \\
\hline Impôt sur le revenu : déduction des pensions pour enfants majeurs étudiants & 280 & 290 & 333 & 265 \\
\hline Impôt sur le revenu : réduction d'impôt pour enfants scolarisés & 400 & 399 & 372 & 216 \\
\hline Impôt sur le revenu : réduction d'impôt pour frais de garde d'enfants de moins de 7 ans & 190 & 200 & 131 & 199 \\
\hline \multicolumn{5}{|l|}{ Autres domaines de politique sociale } \\
\hline Prime pour l'Emploi & 1250 & 2322 & 3127 & 3948 \\
\hline Taxe d'habitation : dégrèvement total pour les titulaires du RMI & 200 & 285 & 295 & 291 \\
\hline \multicolumn{5}{|l|}{ Logement } \\
\hline \multicolumn{4}{|c|}{ Impôt sur le revenu: crédit d'impôt sur les dépenses d'équipement de l'habitation principale en faveur de l'aide aux personnes } & 5 \\
\hline Tax breaks to stimulate private social protection (not including pensions) & 300 & 320 & 382 & 443 \\
\hline \multicolumn{5}{|l|}{ Logement } \\
\hline Impôt sur le revenu : déductions pour la location à des ménages modestes & - & - & - & - \\
\hline Impôt sur les bénéfices : exonération des offices HLM et OPAC & 260 & 270 & 300 & 310 \\
\hline \multicolumn{5}{|l|}{ Autres domaines de politique sociale } \\
\hline Impôt sur le revenu : réduction pour dons aux personnes en difficulté & 40 & 50 & 82 & 133 \\
\hline
\end{tabular}

Sources: Amounts supplied by DREES/INSEE, based on estimates from ACOSS (Agence Centrale des Organismes de Sécurité Sociale) and Drees (comptes de la protection sociale).

Income tax and tax breaks for social purposes: Direction Générale des Impôts, INES model (Insee-Drees). 
DELSA/ELSA/WD/SEM(2011)9

Table Annex I.A.2.1

Detailed information on the impact of the tax system on social expenditure (cont.)

GERMANY

A. Amount of direct tax paid on benefit income (in millions of euros)

\begin{tabular}{|c|c|c|c|c|}
\hline & 2001 & 2003 & 2005 & 2007 \\
\hline Direct taxes and social contributions paid on public cash benefits & 26445.6 & 26856.1 & 30911.6 & 32519.3 \\
\hline Social contributions paid by recipients of benefits (unemployment, disability, etc.) & 16308.0 & 17141.0 & 18728.0 & 19946.0 \\
\hline Income tax on pensions & 5134.0 & 4748.8 & 6900.0 & 8050.0 \\
\hline Social security contributions & 1009.9 & 928.1 & 1322.7 & 1442.9 \\
\hline Soc. Sec. Cont. on pensions for farmers & 255.7 & 227.2 & 266.4 & 273.5 \\
\hline Soc Sec Cont on "versorgungswerke" & 146.7 & 155.9 & 263.1 & 290.6 \\
\hline Supplementary Pensions in Civil Service & 607.4 & 545.0 & 793.2 & 878.8 \\
\hline Progressionsvorbehalt & 1600.0 & 1670.0 & 1895.0 & 2070.0 \\
\hline Tax and social contributions on family wage supplements (public employers) & 2393.7 & 2368.3 & 2065.9 & 1010.4 \\
\hline Direct taxes and social contributions paid on mandatory private cash benefits & 9445.6 & 9271.5 & 8595.2 & 9195.7 \\
\hline Continued wage payments in case of sickness (Entgeltfortzahlung) & 8919.4 & 8746.2 & 8176.5 & 8785.0 \\
\hline Continued Wage Payments: Maternity and parental leave & 526.2 & 525.3 & 418.7 & 410.7 \\
\hline Direct taxes and social contributions paid on voluntary private cash benefits & 2562.7 & 3192.7 & 3856.0 & 4262.1 \\
\hline Taxover BAV (company pension) & 1189.5 & 1305.5 & 1775.0 & 1935.0 \\
\hline Soc. Sec. cont over BAV(company pension) & 1092.8 & 1492.0 & 1713.2 & 2033.2 \\
\hline Tax and soc.sec. cont over other payments & 280.4 & 395.2 & 367.8 & 293.9 \\
\hline
\end{tabular}

Source: Bundes ministerium für Arbeit und Sozialordnung; Calculated while using the nettolohnquote as in the national accounts.

B. Average implicit indirect tax rates of cons umption out of benefit income

Indirect taxes paid out of consumption of cash transfers, in millions of euros

(1) Private final consumption expenditure

(2) Private consumption plus Government consumption minus Government wages

(3) General consumption taxes plus excise duties $(5110+5121)$

5110 General taxes

5121 Excises

(4) Taxes on production sale transfer (5100)

(5) Taxes on Goods and Services (5000)

\begin{tabular}{cccc}
\hline 2001 & 2003 & 2005 & 2007 \\
\hline 1258570 & 1284600 & 1325440 & 1378940 \\
1492580 & 1532210 & 1576100 & 1645440 \\
200521 & 206353 & 205306 & 234198 \\
138935 & 137568 & 140121 & 170387 \\
61586 & 68785 & 65185 & 63811 \\
210991 & 218371 & 217622 & 247858 \\
219602 & 225951 & 226554 & 257019 \\
& & & \\
$\mathbf{1 3 . 4 \%}$ & $\mathbf{1 3 . 5 \%}$ & $\mathbf{1 3 . 0} \%$ & $\mathbf{1 4 . 2 \%}$ \\
$14.7 \%$ & $14.7 \%$ & $14.4 \%$ & $15.6 \%$ \\
$17.4 \%$ & $17.6 \%$ & $17.1 \%$ & $18.6 \%$ \\
\hline
\end{tabular}

Implicit average indirect tax rate on consumption out of benefit income:

\begin{tabular}{|c|c|c|c|c|}
\hline (6) using general consumption taxes plus excise duties (3)/(2) & $13.4 \%$ & $13.5 \%$ & $13.0 \%$ & $14.2 \%$ \\
\hline using a broad concept of the indirect tax base (5)/(2) & $14.7 \%$ & $14.7 \%$ & $14.4 \%$ & $15.6 \%$ \\
\hline using a broad concept of the indirect tax base and ignoring government consumpion (5)/(1) & $17.4 \%$ & $17.6 \%$ & $17.1 \%$ & $18.6 \%$ \\
\hline
\end{tabular}

Source: OECD on-line National Accounts database (http://stats.oecd.org/Index.aspx?DataSetCode=SNA_TABLE1) for lines 1 and 2; and OECD Revenue Statistics database (http://stats.oecd.org/Index.aspx?DataSetCode=REV) for lines 3, 4, and 5. 
DELSA/ELSA/WD/SEM(2011)9

Table Annex I.A.2.1

Detailed information on the impact of the tax system on social expenditure (cont.)

\section{GERMANY}

C. Tax breaks for social purposes (in millions of euros)

\section{Tax breaks similar to cash benefits}

Special expenses for owner-occupied homes

Child component in conjunction with sec. 10 e EStG (sec. $34 \mathrm{f} \mathrm{EStG}$ )

Owner-occupied Homes Premium Law, total

Employee savings premiums for productive investment (sec. 19 a EStG)

Deduction of occupational training expenses

Motor vehicle tax exemption for physically disabled persons

Household allowance

Deduction for extraordinary financial burdens

Deduction for extraordinary financial burdens in special instances

Lump sum allowances for the physically disabled and others

Lump sum care allowance

Childcare expenses (Kinderbetreuungskosten)

Family benefits (child tax credit )

For information: Family benefits (Familienlastenausgleich: tax credit + cash benefits)

Tax breaks to stimulate private social protection (not including pensions)

Exclusion of contributions to health and accident insurance

Donations to (approved) NGOs and political parties

\begin{tabular}{|c|c|c|c|}
\hline 2001 & 2003 & 2005 & 2007 \\
\hline 33327.0 & 36473.0 & 33108.0 & 32483.0 \\
\hline 1733.0 & 430.0 & 30.0 & - \\
\hline 460.0 & 65.0 & 30.0 & - \\
\hline 8050.0 & 10536.0 & 10247.0 & 7722.0 \\
\hline 41.0 & 41.0 & 36.0 & 80.0 \\
\hline 79.0 & 85.0 & 315.0 & 350.0 \\
\hline 135.0 & 130.0 & 140.0 & 146.0 \\
\hline 1100.0 & 1000.0 & 580.0 & 410.0 \\
\hline 465.0 & 475.0 & 650.0 & 730.0 \\
\hline 1094.0 & 659.0 & 655.0 & 610.0 \\
\hline 798.0 & 810.0 & 805.0 & 865.0 \\
\hline 72.0 & 72.0 & 60.0 & 60.0 \\
\hline - & 170.0 & 160.0 & 620.0 \\
\hline 19300.0 & 22000.0 & 19400.0 & 20890.0 \\
\hline$F(32020.7)$ & (36 080) & (36 500) & (36 600) \\
\hline 9480.0 & 9485.0 & 9480.0 & 10580.0 \\
\hline 8400.0 & 8400.0 & 8400.0 & 9300.0 \\
\hline 1080.0 & 1085.0 & 1080.0 & 1280.0 \\
\hline 17520.0 & 18280.0 & 19585.0 & 20270.0 \\
\hline 14300.0 & 15100.0 & 16500.0 & 17400.0 \\
\hline 2300.0 & 2200.0 & 2100.0 & 2000.0 \\
\hline 920.0 & 980.0 & 985.0 & 870.0 \\
\hline
\end{tabular}

a) Donation to political parties are not in the social domain. However, the value of these donations cannot be separately identified, but is considered smaller that the donations to NGOs, and therefore this item is included in the list here.

Source: Bundesministerium fur Arbeit und Sozialordnung, Bundesministerium für Finanzen, Germany. 
DELSA/ELSA/WD/SEM(2011)9

Table Annex I.A.2.1

Detailed information on the impact of the tax system on social expenditure (cont.)

\section{ICELAND}

A. Amount of direct tax paid on benefit income (in millions of Islandic krónur)

\begin{tabular}{|c|c|c|c|c|}
\hline & 2001 & 2003 & 2005 & $2007 *$ \\
\hline $\begin{array}{l}\text { Total tax paid (including soc. sec. cont.) on public transfer income } \\
\text { of which: }\end{array}$ & 4671 & 6075 & 6767 & 8504 \\
\hline - Income tax & 4671 & 6075 & 6767 & 8504 \\
\hline $\begin{array}{l}\text { Total tax paid (including soc. sec. cont.) on private transfer income } \\
\text { of which: }\end{array}$ & 4457 & 5942 & 7407 & 9308 \\
\hline - Income tax & 4457 & 5942 & 7407 & 9308 \\
\hline
\end{tabular}

Source: Ministry of Finance, Economic Department.

B. Average implicit indirect tax rates of cons umption out of benefit income

Indirect taxes paid out of consumption of cash transfers, in millions of Islandic krónur

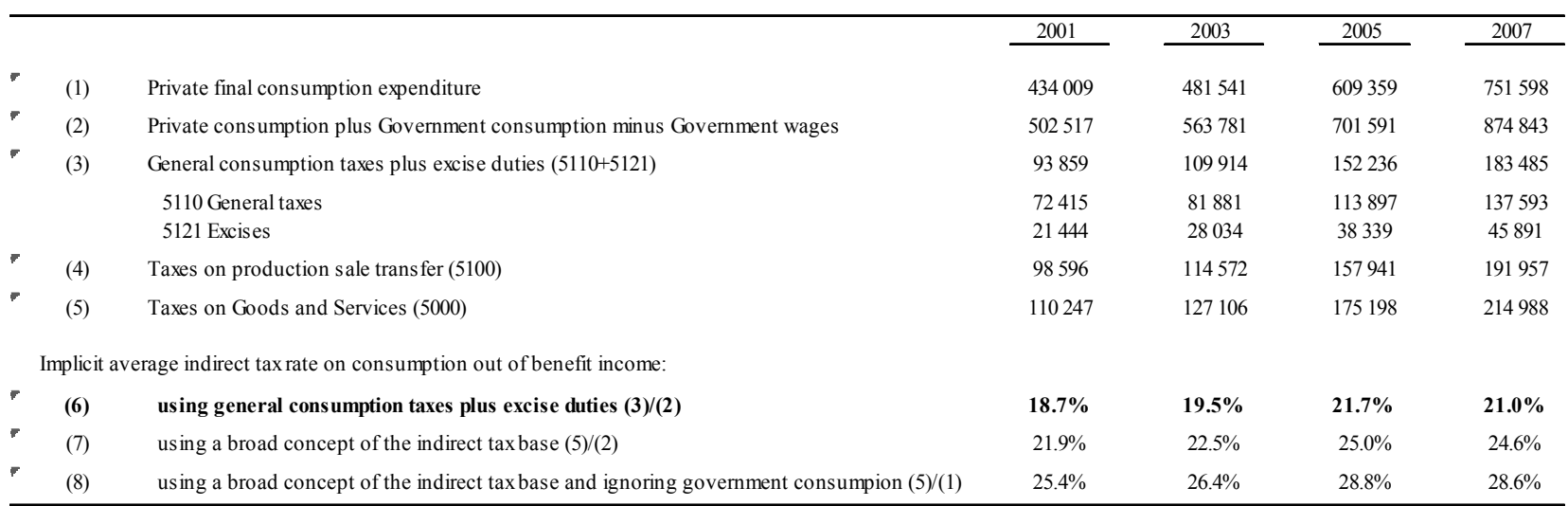

Source: OECD on-line National Accounts database (http://stats.oecd.org/Index.aspx?DataSetCode=SNA TABLE1) for lines 1 and 2; and OECD Revenue Statistics database (http://stats.oecd.org/Index.aspx?DataSetCode=REV) for lines 3, 4, and 5 .

C. Tax breaks for social purposes (in millions of Is landic krónur)

\begin{tabular}{|c|c|c|c|c|}
\hline & 2001 & 2003 & 2005 & $2007 *$ \\
\hline Tax breaks similar to cash benefits & 0 & 0 & 0 & 0 \\
\hline Tax breaks to stimulate private social protection (not including pensions) & 0 & 0 & 0 & 0 \\
\hline \multicolumn{5}{|l|}{ Memorendum item } \\
\hline Tax breaks for pension & 6888 & 8619 & 10596 & 13315 \\
\hline
\end{tabular}

* 2007 figures are estimates based on the 2007/2005 growth rate in public cash social spending, $+25.67 \%$.

Source: Ministry of Finance of Iceland, Economic Department. 
DELSA/ELSA/WD/SEM(2011)9

Table Annex I.A.2.1

Detailed information on the impact of the tax system on social expenditure (cont.)

IRELAND

A. Amount of direct tax paid on benefit income (in millions of euros)

\begin{tabular}{|c|c|c|c|c|}
\hline & 2001 & 2003 & 2005 & 2007 \\
\hline Total tax paid (including soc. sec. cont.) on transfer income & 299.4 & 331.6 & 371.3 & 470.6 \\
\hline Social Security Pension & 267.6 & 291.4 & 326.7 & 408.4 \\
\hline Social Security Disability Benefit & 20.6 & 23.2 & 27.8 & 33.8 \\
\hline Social security Unemployment Benefit & 11.2 & 17.0 & 16.8 & 28.4 \\
\hline
\end{tabular}

Source: Ireland Revenue, Income Tax Returns for 2003, 2004, 2006 (SAS).

B. Average implicit indirect tax rates of cons umption out of benefit income

Indirect taxes paid out of consumption of cash transfers, in millions of euros)

(1) Private final consumption expenditure

(2) Private consumption plus Government consumption minus Government wages

\begin{tabular}{|c|c|c|c|}
\hline & & & \\
\hline 55675 & 65249 & 74632 & 88882 \\
\hline 62760 & 73627 & 83419 & 100215 \\
\hline 11675 & 14193 & 17473 & 20085 \\
\hline 7546 & 9524 & 12134 & 14156 \\
\hline 4129 & 4669 & 5339 & 5930 \\
\hline 11830 & 14337 & 17618 & 20211 \\
\hline 12389 & 15032 & 18435 & 21185 \\
\hline
\end{tabular}

12389

$15032 \quad 1843$

21185

Implicit average indirect tax rate on consumption out of benefit income:

(6) using general consumption taxes plus excise duties (3)/(2)

(7) using a broad concept of the indirect tax base (5)/(2)

$\begin{array}{llll}18.6 \% & 19.3 \% & 20.9 \% & 20.0 \%\end{array}$

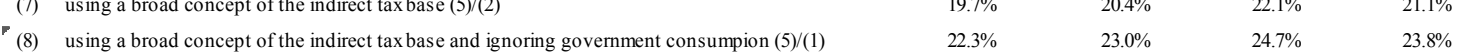

Source: OECD on-line National Accounts database (http://stats.oecd.org/Index.aspx?DataSetCode=SNA_TABLE1) for lines 1 and 2; and OECD Revenue Statistics database (http://stats.oecd.org/Index.aspx?DataSetCode=REV) for lines 3, 4, and 5 .

C. Tax breaks for social purposes (in millions of euros)

\begin{tabular}{|c|c|c|c|c|}
\hline & 2001 & $2003 *$ & $2005 *$ & $2007 *$ \\
\hline Tax breaks similar to cash benefits & 196.6 & 319.3 & 395.6 & 420.4 \\
\hline Exemption of statutory redundancy payments & 8.7 & 25.1 & 76.9 & - \\
\hline Widowed person's allowance (data do not cover non-tax payers) & 82.6 & 122.3 & 125.7 & 155.2 \\
\hline Additional allowance to widowed person in year of bereavement & 4.2 & 6.1 & 4.9 & 4.5 \\
\hline Additional bereavement allowance to widowed parent & 3.9 & 5.6 & 4.0 & 4.9 \\
\hline Additional personal allowance for one parent family & 91.5 & 137.8 & 150.8 & 186.1 \\
\hline Additional allowance for incapacitated child & 2.7 & 4.4 & 5.0 & 16.0 \\
\hline Dependent relative allowance & 0.8 & 1.1 & 1.1 & 1.4 \\
\hline Person taking care of incapacitated taxpayer & 0.4 & 0.6 & 1.3 & 2.8 \\
\hline Donations to approved bodies & 1.8 & 16.3 & 25.9 & 49.5 \\
\hline Tax breaks to stimulate private social protection (not including pensions) & 205.5 & 226.6 & 330.3 & 430.8 \\
\hline Relief in respect of medical insurance premiums & 168 & 161.7 & 218.2 & 260.5 \\
\hline Health expenses relief & 36 & 63.2 & 109.6 & 167.2 \\
\hline $\begin{array}{l}\text { Contributions under permanent health benefit schemes after deduction of tax on benefits received } \\
\text { Exemption of income of charities, colleges, hospitals, schools, friendly societies, etc. }\end{array}$ & 1.5 & 1.7 & 2.5 & 3.1 \\
\hline \multicolumn{5}{|l|}{ Memorendum item } \\
\hline Tax breaks to pensions & 2009.5 & 2708.9 & 2240.0 & 2299.2 \\
\hline Employee's Contributions to Approved Superannuation Schemes: & 388.7 & 563.3 & 540.0 & 543.3 \\
\hline Employer's Contributions to Approved Superannuation Schemes ** & 497.7 & 623.1 & 120.0 & 120.0 \\
\hline Exemption of Net Income of Approved Superannuation Funds & 938.4 & 1271.6 & 1200.0 & 1200.0 \\
\hline Retirement Annuity Premiums (Individual private pensions) & 184.7 & 250.9 & 380.0 & 435.9 \\
\hline
\end{tabular}

* TBSPs are for 2002 instead of 2003, 2004 instead of 2005, and 2006 intstead of 2007.

** Newly available 2006 aggregate data on contributions to pension schemes by employers and employees arising from a P35 initiative introduced on foot of provisions that were included in Finance Act 2004 with a view to improving data quality have allowed estimates of the cost of tax for private pension provision for 2006 to be made. Further work is ongoing to provide similar estimates for 2005. As similar data sources would not be available for previous years, it is not possible to provide costings on a similar basis for those years.

Source: Office of the Revenue Commissioners, Statistical Reports. 
DELSA/ELSA/WD/SEM(2011)9

Table Annex I.A.2.1

Detailed information on the impact of the tax system on social expenditure (cont.)

TTALY

A. Amount of direct tax paid on benefit income (in millions of euros)

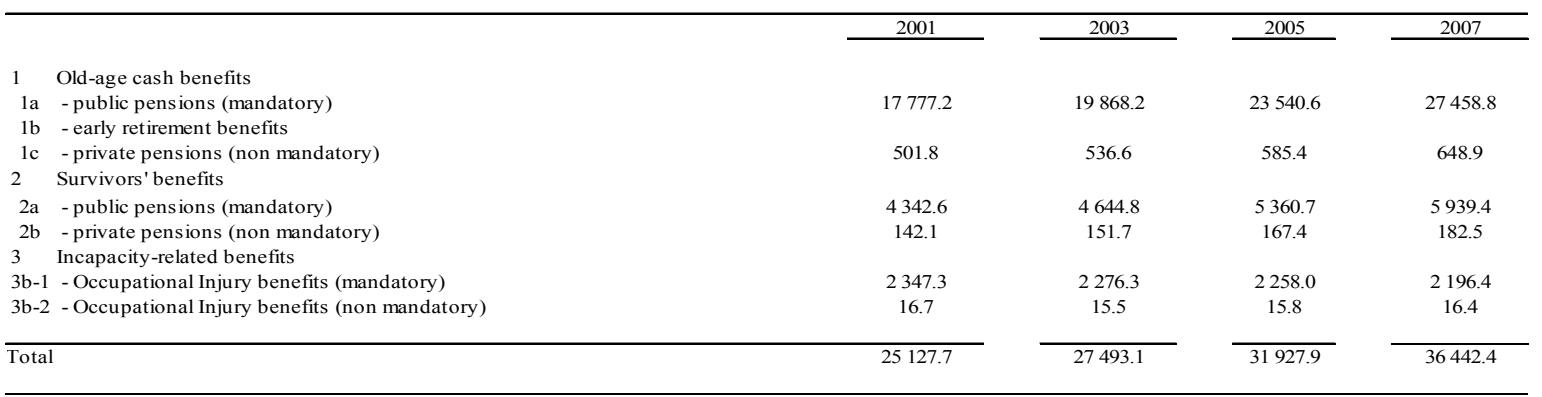

Source: Official administrative information from National Institute for Social Security (INPS).

B. Average implicit indirect tax rates of cons umption out of benefit income

Indirect taxes paid out of consumption of cash trans fers, in millions of euros

\begin{tabular}{|c|c|c|c|c|c|}
\hline & & 2001 & 2003 & 2005 & 2007 \\
\hline (1) & Private final consumption expenditure & 737680 & 789026 & 843978 & 907546 \\
\hline & Private consumption plus Government consumption minus Government wages & 842890 & 907219 & 978254 & 1047738 \\
\hline & General consumption taxes plus excise duties $(5110+5121)$ & 109277 & 111784 & 117712 & 128527 \\
\hline & $\begin{array}{l}5110 \text { General taxes } \\
5121 \text { Excises }\end{array}$ & $\begin{array}{l}78056 \\
31221\end{array}$ & $\begin{array}{l}79099 \\
32685\end{array}$ & $\begin{array}{l}85317 \\
32395\end{array}$ & $\begin{array}{l}95623 \\
32904\end{array}$ \\
\hline (4) & Taxes on production sale transfer $(5100)$ & 125750 & 128966 & 138938 & 152930 \\
\hline \multicolumn{6}{|c|}{ Implicit average indirect tax rate on consumption out of benefit income: } \\
\hline (6) & using general cons umption taxes plus excise duties (3)/(2) & $13.0 \%$ & $12.3 \%$ & $12.0 \%$ & $12.3 \%$ \\
\hline (7) & using a broad concept of the indirect tax base (5)/(2) & $16.7 \%$ & $15.8 \%$ & $15.8 \%$ & $16.2 \%$ \\
\hline$(8)$ & using a broad concept of the indirect tax base and ignoring government consumpion ( 5 & $19.0 \%$ & $18.2 \%$ & $18.3 \%$ & $18.7 \%$ \\
\hline
\end{tabular}

Source: OECD on-line National Accounts database (http://stats.oecd.org/Indexaspx?DataSetCode=SNA_TABLE1) for lines 1 and 2; and OECD Revenue Statistics database (http://stats.oecd.org/Index.aspx?DataSetCode=REV) for lines 3, 4, and 5.

C. Tax breaks for social purposes (in millions of euros)

\begin{tabular}{|c|c|c|c|c|}
\hline & 2001 & 2003 & 2005 & 2007 \\
\hline \multicolumn{5}{|l|}{ Taxcredits: } \\
\hline Healthcare expenses for disabled (1) & 7.1 & & & \\
\hline Other expenses for disabled (vehicles, dogs, $\ldots$ ) & 17.8 & & & \\
\hline Education expenses & 188.5 & 221.6 & 249.6 & 282.5 \\
\hline \multicolumn{5}{|l|}{ Tax deductions: } \\
\hline Tax breaks to stimulate private social protection (not including pensions) & 1691.9 & 1458.2 & 1236.0 & 1064.4 \\
\hline \multicolumn{5}{|l|}{ Tax credits: } \\
\hline Donations to ONLUS & 24.9 & 36.4 & 38.7 & 38.7 \\
\hline Contributions to mutual assistance associations & 4.8 & & & \\
\hline Contributions to health and accident insurance & 1662.2 & 1421.8 & 1197.3 & 1025.6 \\
\hline
\end{tabular}

1) For years 2003, 2005 and 2007 healthcare expenses include healthcare expenses for disabled

2) The estimate is based on the amount of the relevant deductions as recorded in all individual tax returns, distributed in 30 income class. The revenue foregone is calculated applying to the deductions in each income class the corresponding P.I.T. average implicit tax rate.

3) The tax break in years 2001, 2003, 2005 and 2007 are the reduction of tax rate by $1.5 \%$. The estimate is based on administrative data for the tax revenue from income of pension funds. The tax revenue without the tax break is estimated by applying the ordinary tax rate of $12.5 \%$ to the 2001, 2003, 2005 and 2007 taxable income. The revenue foregone is then calculated as the difference between this estimated tax revenue and the actual tax revenue.

Source: Official administrative in formation from National Institute for Social Security (INPS). 
DELSA/ELSA/WD/SEM(2011)9

Table Annex I.A.2.1

Detailed information on the impact of the tax system on social expenditure (cont.)

JAPAN

A. Amount of direct tax paid on benefit income (in millions of yen)

\begin{tabular}{|c|c|c|c|c|}
\hline & 2001 & 2003 & 2005 & 2007 \\
\hline \multicolumn{5}{|c|}{$\begin{array}{l}\text { Income tax rate of } 0.01 \%+\text { Health insurance contribution of } 2.72 \%+\text { Long term care insurance } \\
\text { contribution of } 1.82 \% \text { in } 2005\end{array}$} \\
\hline - public old pensions & 1097156.9 & 1167918 & 1994849 & 2082382 \\
\hline - mandatory private old age pensions & 83106.0 & 77246 & 89074 & 91302 \\
\hline - voluntary private old age pensions & 392625.3 & 343467 & 305574 & 354772 \\
\hline
\end{tabular}

Source: Ministry of Finance of Japan and estimates from National Institute of Population and Social Security Research.

B. Average implicit indirect tax rates of cons umption out of benefit income Indirect taxes paid out of consumption of cash trans fers, in millions of yen

\begin{tabular}{|c|c|c|c|c|c|}
\hline & & 2001 & 2003 & 2005 & 2007 \\
\hline$(1)$ & Private final consumption expenditure & 284216600 & 281791000 & 285935600 & 292523200 \\
\hline$(2)$ & Private consumption plus Government consumption minus Government wages & 338483700 & 337985900 & 345042500 & 353089300 \\
\hline \multirow[t]{2}{*}{ (3) } & General consumption taxes plus excise duties $(5110+5121)$ & 21985200 & 21792400 & 22705400 & 22214700 \\
\hline & $\begin{array}{l}5110 \text { General taxes } \\
5121 \text { Excises }\end{array}$ & $\begin{array}{c}12241600 \\
9743600\end{array}$ & $\begin{array}{l}12106400 \\
9686000\end{array}$ & $\begin{array}{c}13134600 \\
9570800\end{array}$ & $\begin{array}{c}12841100 \\
9373600\end{array}$ \\
\hline (4) & Taxes on production sale transfer $(5100)$ & 22990800 & 22731900 & 23722200 & 23240700 \\
\hline$(5)$ & Taxes on Goods and Services (5000) & 26053000 & 25792300 & 26786000 & 26255800 \\
\hline \multicolumn{6}{|c|}{ Implicit average indirect tax rate on consumption out of benefit income: } \\
\hline (6) & using general consumption taxes plus excise duties (3)/(2) & $6.5 \%$ & $6.4 \%$ & $6.6 \%$ & $6.3 \%$ \\
\hline$(7)$ & using a broad concept of the indirect tax base (5)/(2) & $7.7 \%$ & $7.6 \%$ & $7.8 \%$ & $7.4 \%$ \\
\hline$(8)$ & using a broad concept of the indirect tax base and ignoring government consumpion (5)/(1) & $9.2 \%$ & $9.2 \%$ & $9.4 \%$ & $9.0 \%$ \\
\hline
\end{tabular}

Source: OECD on-line National Accounts database (http://stats.oecd.org/Index.aspx?DataSetCode=SNA_TABLE1) for lines 1 and 2; and OECD Revenue Statistics database (http://stats.oecd.org/Index.aspx?DataSetCode=REV) for lines 3, 4, and 5.

C. Tax breaks for social purposes (in millions of yen)

\begin{tabular}{|c|c|c|c|c|}
\hline & 2001 & 2003 & 2005 & 2007 \\
\hline TBSPs similar to cas $h$ benefits & 2800000 & 2720000 & 2600000 & 2700000 \\
\hline Deduction for dependent family other than spouses (General taxation) & 2100000 & 2370000 & 2400000 & 2600000 \\
\hline Deduction for handicapped, survivors and working students (General taxation) & 700000 & 140000 & 100000 & 100000 \\
\hline Deduction for the elderly * & .. & 210000 & 100000 & - \\
\hline Deduction for medical expenses & .. & .. & .. & .. \\
\hline Deduction for retirement income & .. & .. & .. & .. \\
\hline Tax breaks to stimulate private social protection (not including pensions) & 110000 & 132000 & 129000 & 136000 \\
\hline Special treatment of fees for medical care under the social insurance system & 28000 & 25000 & 25000 & 31000 \\
\hline Enterprise tax exemption of medical care under the social insurance system & 82000 & 107000 & 104000 & 105000 \\
\hline Tax exemption for social welfare juridical persons & .. & .. & .. & .. \\
\hline \multicolumn{5}{|l|}{ Memorendum item } \\
\hline Tax breaks to pensions & 3620000 & 3040000 & 3190000 & 3640000 \\
\hline $\begin{array}{l}\text { - Deduction of contributions to private pensions (e.g occupational pension plans, individual } \\
\text { retirement accounts, RRSPs, Superannuation, etc) }\end{array}$ & - & 2580000 & 2890000 & 3330000 \\
\hline - Non-taxation of investment of private pension funds & - & 460000 & 300000 & 310000 \\
\hline
\end{tabular}

* The amount of the "Deduction for the elderly" is not available in 2001.

Source: Information supplied by the Ministry of Finance of Japan. 
DELSA/ELSA/WD/SEM(2011)9

Table Annex I.A.2.1

Detailed information on the impact of the tax system on social expenditure (cont.)

KOREA

A. Amount of direct tax paid on benefit income (in millions wons)

\begin{tabular}{|c|c|c|c|c|c|c|c|}
\hline & 2001 & 2002 & 2003 & 2004 & 2005 & 2006 & 2007 \\
\hline \multicolumn{8}{|l|}{ Public social expenditure } \\
\hline Social contributions & 34842 & 49669 & 51109 & 115549 & 131947 & 161743 & 210991 \\
\hline Mandatory private social expenditure & 208942 & 189391 & 216186 & 258537 & 260528 & 287248 & 322893 \\
\hline Voluntary private social expenditure & - & - & - & - & - & - & - \\
\hline
\end{tabular}

Source: See table $\mathrm{C}$ below.

B. Average implicit indirect tax rates of consumption out of benefit income

Indirect taxes paid out of consumption of cash transfers, in millions of wons

\begin{tabular}{|c|c|c|c|c|c|}
\hline & & 2001 & 2003 & 2005 & 2007 \\
\hline (1) & Private final consumption expenditure & 364251400 & 420098600 & 465430500 & 530264100 \\
\hline (2) & Private consumption plus Government consumption minus Government wages & 404232000 & 468019300 & 522951200 & 602100500 \\
\hline \multirow[t]{2}{*}{ (3) } & General consumption taxes plus excise duties $(5110+5121)$ & 48448000 & 57438000 & 61006000 & 68822000 \\
\hline & $\begin{array}{l}5110 \text { General taxes } \\
5121 \text { Excises }\end{array}$ & $\begin{array}{l}25835000 \\
22613000\end{array}$ & $\begin{array}{l}33447000 \\
23991000\end{array}$ & $\begin{array}{l}36118000 \\
24888000\end{array}$ & $\begin{array}{l}40942000 \\
27880000\end{array}$ \\
\hline (4) & Taxes on production sale transfer $(5100)$ & 57430000 & 66354000 & 69069000 & 78414000 \\
\hline$(5)$ & Taxes on Goods and Services $(5000)$ & 59377000 & 68197000 & 71041000 & 80861000 \\
\hline \multicolumn{6}{|c|}{ Implicit average indirect tax rate on consumption out of benefit income: } \\
\hline (6) & using general consumption taxes plus excise duties (3)/(2) & $12.0 \%$ & $12.3 \%$ & $11.7 \%$ & $11.4 \%$ \\
\hline (7) & using a broad concept of the indirect tax base (5)/(2) & $14.7 \%$ & $14.6 \%$ & $13.6 \%$ & $13.4 \%$ \\
\hline (8) & $\begin{array}{l}\text { using a broad concept of the indirect tax base and ignoring government } \\
\text { consumption }(5) /(1)\end{array}$ & $16.3 \%$ & $16.2 \%$ & $15.3 \%$ & $15.2 \%$ \\
\hline
\end{tabular}

Source: OECD on-line National Accounts database (http://stats.oecd.org/Index.aspx?DataSetCode=SNA_TABLE1) for lines 1 and 2; and OECD Revenue Statistics database (http://stats.oecd.org/Index.aspx?DataSetCode=REV) for lines 3,4 , and 5 .

C. Tax breaks for social purposes (in millions of wons)

\begin{tabular}{|c|c|c|c|c|c|c|c|}
\hline & 2001 & 2002 & 2003 & 2004 & 2005 & 2006 & 2007 \\
\hline Tax breaks similar to cash benefits & 2024445 & 3990206 & 4709134 & 4839672 & 5658833 & 6106589 & 7120604 \\
\hline Tax abatement for non-profit corporation & 20793 & 24949 & 30480 & 32708 & 44377 & 56721.534 & 104645 \\
\hline for social welfare support institution & 186937 & 197893 & 202616 & 217781 & 179389 & 196207.78 & 213679 \\
\hline for imported goods for the disabled & 7744 & 14369 & 26292 & 31825 & 8190 & 8405.504 & 9049 \\
\hline for the cars used by the disabled & 178192 & 72769 & 65743 & 33059 & 37776 & 67754 & 54731 \\
\hline Income deduction (tax credit) & 1630780 & 3680226 & 4384003 & 4524300 & 5389100 & 5777500 & 6738500 \\
\hline Tax breaks to stimulate private social protection (not including pensions) & 0 & 0 & 0 & 0 & 0 & 0 & 0 \\
\hline
\end{tabular}

Gho, K.H, Chang Y.S and J.W, Kang (2009), Estimation of Social Expenditure in Korea on the Basis of the OECD Guidelines: 1995 - 2007 (in Korean only), Korean Institute for Health and Social Affairs (KIHASA), Korea. 
DELSA/ELSA/WD/SEM(2011)9

Table Annex I.A.2.1

Detailed information on the impact of the tax system on social expenditure (cont.)

Luxembourg

A. Average Itemised Tax Rates (AITR \%)

\begin{tabular}{|c|c|c|c|c|c|c|c|c|}
\hline & & ESSPROS scheme(s) & ESSPROS category (1) & 2003 & 2004 & 2005 & 2006 & 2007 \\
\hline 1 & Old-age cash benefits & & & & & & & \\
\hline 1a & - public pensions (2) & 3: Pension scheme & 1131111 & $8.3 \%$ & $8.7 \%$ & $9.4 \%$ & $9.7 \%$ & $10.5 \%$ \\
\hline $1 \mathrm{~b}$ & - early retirement benefits (public) & 3: Pension scheme & 1131112 & $8.0 \%$ & $8.6 \%$ & $9.4 \%$ & $9.8 \%$ & $10.5 \%$ \\
\hline 1c1 & -private pensions (3) & 17+18+19+20: Special Pension scheme & 1131111 & $17.3 \%$ & $17.6 \%$ & $18.6 \%$ & $19.5 \%$ & $20.3 \%$ \\
\hline 1c2 & - early retirement benefits (private) & 17+18+19+20: Special Pension scheme & 1131112 & $18.3 \%$ & $18.7 \%$ & $19.6 \%$ & $19.7 \%$ & $20.6 \%$ \\
\hline$\underline{2}$ & Old-age cash benefits (survivor) & & & & & & & \\
\hline $2 \mathrm{a}$ & -public pensions (2) & 3: Pension scheme & 1141111 & $5.7 \%$ & $5.9 \%$ & $6.5 \%$ & $6.9 \%$ & $7.5 \%$ \\
\hline $2 \mathrm{~b}$ & - private pensions (3) & $17+18+19+20$ : Special Pension scheme & 1141111 & $10.6 \%$ & $11.3 \%$ & $12.1 \%$ & $12.2 \%$ & $13.2 \%$ \\
\hline 3 & Incapacity-related benefits & & & & & & & \\
\hline $3 \mathrm{a} 1$ & - Disability pensions (public) & 3: Pension scheme & 1121111 & $5.7 \%$ & $6.0 \%$ & $6.5 \%$ & $6.7 \%$ & $7.2 \%$ \\
\hline $3 \mathrm{a} 2$ & - Disability pensions (private) & 17+18+19+20: Special Pension scheme & 1121111 & $13.2 \%$ & $13.4 \%$ & $13.7 \%$ & $12.5 \%$ & $13.6 \%$ \\
\hline $3 b$ & - Occupational Injury benefits & 4: Occupational injury & 1121111 & $0.0 \%$ & $0.0 \%$ & $0.0 \%$ & $0.0 \%$ & $0.0 \%$ \\
\hline $4^{3 c}$ & $\begin{array}{l}\text { - Sickness payments (4) } \\
\text { Family cash benefits }\end{array}$ & 4: Occupational injury +2 : Health care and paid sick leave & 1111111 & $16.7 \%$ & $16.9 \%$ & $17.3 \%$ & $17.5 \%$ & $18.3 \%$ \\
\hline $4 \mathrm{a}$ & -Family benefits & 1: Family allowances & $1151113+1151121+1151123$ & $0.0 \%$ & $0.0 \%$ & $0.0 \%$ & $0.0 \%$ & $0.0 \%$ \\
\hline $4 \mathrm{~b}$ & - Maternity and parental leave payments & 1: Family allowances +2 : Health care and paid sick leave & $1151112+1151114+1151111$ & $14.4 \%$ & $14.6 \%$ & $14.8 \%$ & $15.1 \%$ & $15.9 \%$ \\
\hline $6^{4 c}$ & $\begin{array}{l}\text { - Sole parent benefits } \\
\text { Unemployment }\end{array}$ & & & & & & & \\
\hline $8^{6 a}$ & $\begin{array}{l}\text { - unemployment insurance benefit } \\
\text { Other contingencies }\end{array}$ & 5: Employment measures & $1161111+1161113+1161115$ & $16.6 \%$ & $16.5 \%$ & $16.8 \%$ & $16.7 \%$ & $17.4 \%$ \\
\hline $9^{8 \mathrm{a}}$ & $\begin{array}{l}\text { - Low Income benefits } \\
\text { Wage income (5) }\end{array}$ & 10: national solidarity & $1151114+1182111$ & $\begin{array}{r}6.2 \% \\
22.7 \% \\
\end{array}$ & $\begin{array}{r}6.3 \% \\
23.1 \% \\
\end{array}$ & $\begin{array}{r}6.3 \% \\
23.7 \% \\
\end{array}$ & $\begin{array}{r}5.9 \% \\
24.1 \% \\
2\end{array}$ & $\begin{array}{r}6.5 \% \\
25.0 \% \\
\end{array}$ \\
\hline 10 & Wage income + transferts & & & $19.8 \%$ & $20.2 \%$ & $20.8 \%$ & $21.2 \%$ & $22.2 \%$ \\
\hline
\end{tabular}

1) Correspondance of EUROSTAT ESSPROS scheme and category.

2) Public transfer income concerns all cash benefits paid by general government (different levels of government and social security institutions). Other social benefits, e.g. occupational pension payments, are considered private.

3) All pension income (old-age cash benefits) paid by the state or a social security fund.

4) If sickness benefits paid through social insurance funds (please indicate whether or not his covers maternity and parental benefits in your data-set).

5) This category is included for reference purposes, but can be used to estimate tax paid by recipients of continued wage payments in case of absence because of illness.

Source: Microsimulation de l'Inspection générale de la sécurité sociale (IGSS) du Luxembourg (www.mss.public.lu).

B. Taux indirect implicite moyen d'imposition sur la consommation

Impôts indirects payés sur la consommation des prestations en espèces, en millions de la monnaies nationale

(1) Dépense privée de consommation finale des ménages

\begin{tabular}{|c|c|c|}
\hline 2003 & 2005 & 2007 \\
\hline 9731 & 10749 & 11826 \\
\hline 11898 & 13372 & 14733 \\
\hline 2628 & 3204 & 3484 \\
\hline 1464 & 1863 & 2090 \\
\hline 1164 & 1342 & 1394 \\
\hline 2682 & 3262 & 3546 \\
\hline 2713 & 3295 & 3614 \\
\hline $22.1 \%$ & $24.0 \%$ & $23.7 \%$ \\
\hline $22.8 \%$ & $24.6 \%$ & $24.5 \%$ \\
\hline $27.9 \%$ & $30.7 \%$ & $30.6 \%$ \\
\hline
\end{tabular}

Consommation des ménages et des

administrations publiques moins les

(2) salaires des administrations publiques

(3) Impôts sur la consommation plus droits d'accise $(5110+5121)$ 5110 Impôts généraux 5121 Accises

(4) Impôts sur production, vente, trans fert (5100)

(5) Impôts sur les biens et services (5000)

Taux indirect implicite moyen d'imposition sur la consommation :

- (6) par les impôts sur la consommation et les droits d'accise (3)/(2)

- (7) par un concept plus large de base d'imposition (5)/(2) par un concept plus large de base d'imposition et en ignorant la

consommation des administrations

(8) publiques $(5) /(1)$

Source: OECD on-line National Accounts database (http://stats.oecd.org/Index.aspx?DataSetCode=SNA_TABLE1) for lines 1 and 2; and OECD Revenue Statistics database (http://stats.oecd.org/Index.aspx? DataSetCode=REV) for lines 3, 4, and 5 .

C. Avantages fiscaux à finalité sociale (en millions d'euros)

Aucun. 
DELSA/ELSA/WD/SEM(2011)9

Table Annex I.A.2.1

Detailed information on the impact of the tax system on social expenditure (cont.)

MEXICO

A. Amount of direct tax paid on benefit income (in millions of pesos)

Almost all the income of individuals from social programs is exempt, as stated in article 109 of the Income Tax Law of 2003-2005 (article 177 in 2001), and there are no revenue statistics of the portion of such income that is considered taxable income.

B. Average implicit indirect tax rates of consumption out of benefit income

Indirect taxes paid out of consumption of cash transfers, in millions of pesos)

(1) Private final consumption expenditure

(2) Private consumption plus Government consumption minus Government wages

(3) General consumption taxes plus excise duties $(5110+5121)$ 5110 General taxes 5121 Excises

(4) Taxes on production sale transfer (5100)

(5) Taxes on Goods and Services (5000)

\begin{tabular}{|c|c|c|c|}
\hline 2001 & 2003 & 2005 & 2007 \\
\hline 4306162 & 5042755 & 6141604 & 7316008 \\
\hline $4395 \quad 172$ & 5247724 & 6353356 & 7575007 \\
\hline 323706 & 376888 & 373718 & 456021 \\
\hline 208408 & 254433 & 318432 & 409013 \\
\hline 115298 & 122455 & 55286 & 47008 \\
\hline 550184 & 675640 & 928981 & 1042372 \\
\hline 561704 & 689054 & 944881 & 1063583 \\
\hline $7.4 \%$ & $7.2 \%$ & $5.9 \%$ & $6.0 \%$ \\
\hline $12.8 \%$ & $13.1 \%$ & $14.9 \%$ & $14.0 \%$ \\
\hline $13.0 \%$ & $13.7 \%$ & $15.4 \%$ & $14.5 \%$ \\
\hline
\end{tabular}

Implicit average indirect tax rate on consumption out of benefit income:

(6) using general consumption taxes plus excise duties (3)/(2)

(7) using a broad concept of the indirect tax base (5)/(2)

(8) using a broad concept of the indirect tax base and ignoring government consumpion (5)/(1)

$13.0 \%$

$13.7 \%$

$15.4 \%$

$1.0 \%$

Source: OECD on-line National Accounts database (http://stats.oecd.org/Indexaspx?DataSetCode=SNA_TABLE1) for lines 1 and 2; and OECD Revenue Statistics database

(http://stats.oecd.org/Index.aspx?DataSetCode=REV) for lines 3,4, and 5 .

C. Tax breaks for social purposes (in millions of pesos)

Tax breaks Similar to cash benefits

- Fiscal subsidy (Art. 80-A of ITL 2001, Art. 114,178 of 2005 ITL)

(wastable tax credit eliminating the existing inequity in the employees' income tax by reducing the tax burden

of those workers that receive a minor proportion of exempt fringe benefits, consequently reducing the

difference with those who earn the same level of income and obtain a significant percentage of exempt fringe

benefits)

- Social prevision services (section VI Art.77 of ITL 2001, Art.109 of 2005 ITL) **

(includes income received as subsidies for disabilities, educational scholarships for workers or their children, day care, cultural and sports activities, and other, similar social benefits that are given across the board, in accordance with laws or labor contracts)

Tax breaks to stimulate private social protection (not including pensions)

- Income from saving funds (section VIII Art.77 of ITL 2001, Art.109 of 2005 ITL)

- Reimbursement of medical, dental and funeral expenses (section IV Art.77 of ITL 2001, Art.109 of 2005 ITL)

- Donations to (approved) institutions (Art.31 and 176 section III of ITL 2005)

\section{Memorendum item}

Tax breaks to pensions

- Retirement and pensions (Art. 109 section III of 2005 ITL) **

- Deduction of employers contributions to retirement and pension funds (voluntary contributions) (Art.29 section VII of 2005 ITL)

\begin{tabular}{|c|c|c|c|}
\hline 2001 * & 2003 & 2005 & 2007 \\
\hline 66590.0 & 50320.0 & 77533.2 & 99893.2 \\
\hline 52910.0 & 34108.0 & 58938.4 & 77069.2 \\
\hline 13680.0 & 16212.0 & 18594.8 & 22824.0 \\
\hline
\end{tabular}

* TBSPs are for 2002 as 2001 figures are not available.

** 2004 figure (17 995.5 for Social prevision services, and 2342 for Retirement and pensions) asjusted with 2005 inflation.

Source: Tax Expenditures Budget 2002-2009 (Presupuesto de Gastos Fiscales 2002-2009) 
DELSA/ELSA/WD/SEM(2011)9

Table Annex I.A.2.1

Detailed information on the impact of the tax sys tem on social expenditure (cont.)

NETHERLANDS

A. Average Itemised Tax Rates (AITR \%)

\begin{tabular}{|c|c|c|c|c|c|}
\hline & & 2001 & 2003 & 2005 & $2007 *$ \\
\hline & Old-age cash benefits & 14.6 & - & - & - \\
\hline 1a & -public pensions & 7.1 & 13.8 & 13.8 & 9.2 \\
\hline $1 \mathrm{~b}$ & - early retirement benefits & 27.9 & 32.3 & 32.9 & 26.8 \\
\hline $1 \mathrm{c}$ & - private pensions & 16.8 & 21.5 & 22.1 & 17.0 \\
\hline 2 & Survivors' benefits & 25.3 & & & \\
\hline $2 \mathrm{a}$ & - public pensions & 23.5 & 24.1 & 24.3 & 25.5 \\
\hline $2 b$ & - private pensions & 29.5 & 24.1 & 24.3 & 25.5 \\
\hline 3 & Incapacity-related benefits & & & & \\
\hline $3 a$ & - Disability pensions & 20.4 & 27.1 & 27.6 & 27.6 \\
\hline 6 & Unemployment & & & & \\
\hline $6 \mathrm{a}$ & - unemployment insurance benefit & 21.6 & 25.5 & 25.8 & 24.2 \\
\hline $6 \mathrm{~b}$ & - unemployment assistance benefit & 14.6 & 16.5 & 16.5 & 16.2 \\
\hline 8 & Other contingencies & & & & \\
\hline $8 \mathrm{a}$ & - Low Income benefits & 14.0 & 16.5 & 16.5 & 16.2 \\
\hline 9 & Wage income & 25.5 & 32.5 & 33.4 & 27.0 \\
\hline
\end{tabular}

* 2007 tax rates in italics are Secretariat estimes.

The micro-simulation model used is based on annual tax data from a representative sample of taxpayers (220 000 individuals, of whom 150000 have income, or $1.5 \%$ of the taxpaying population). These tax data mainly comprise information from income and wage tax returns and assessments. It normally takes three years before sufficient tax data are available and the simulation model is adjusted, and before reliable up-to-date estimates can be made for current and future years.

Source: Ministry of Finance, The Netherlands.

B. Average implicit indirect tax rates of consumption out of benefit income Indirect taxes paid out of consumption of cash transfers, in millions of euros

\begin{tabular}{|c|c|c|c|c|c|}
\hline & & 2001 & 2003 & 2005 & 2007 \\
\hline$(1)$ & Private final consumption expenditure & 224244 & 238103 & 250343 & 264099 \\
\hline (2) & Private consumption plus Government consumption minus Government wages & 282809 & 306856 & 322485 & 355618 \\
\hline \multirow[t]{2}{*}{ (3) } & General consumption taxes plus excise duties $(5110+5121)$ & 46952 & 49440 & 55371 & 59857 \\
\hline & $\begin{array}{l}5110 \text { General taxes } \\
5121 \text { Excises }\end{array}$ & $\begin{array}{l}32509 \\
14443\end{array}$ & $\begin{array}{l}34754 \\
14686\end{array}$ & $\begin{array}{l}38566 \\
16805\end{array}$ & $\begin{array}{l}42216 \\
17641\end{array}$ \\
\hline (4) & Taxes on production sale transfer $(5100)$ & 48060 & 50844 & 56892 & 60742 \\
\hline$(5)$ & Taxes on Goods and Services (5000) & 52754 & 55918 & 62714 & 63603 \\
\hline \multicolumn{6}{|c|}{ Implicit average indirect tax rate on consumption out of benefit income: } \\
\hline (6) & using general cons umption taxes plus excise duties $(3) /(2)$ & $16.6 \%$ & $16.1 \%$ & $17.2 \%$ & $16.8 \%$ \\
\hline$(7)$ & using a broad concept of the indirect tax base (5)/(2) & $18.7 \%$ & $18.2 \%$ & $19.4 \%$ & $17.9 \%$ \\
\hline$(8)$ & using a broad concept of the indirect tax base and ignoring government consumpion (5)/(1) & $23.5 \%$ & $23.5 \%$ & $25.1 \%$ & $24.1 \%$ \\
\hline
\end{tabular}

Source: OECD on-line National Accounts database (http://stats.oecd.org/Index.aspx?DataSetCode=SNA_TABLE1) for lines 1 and 2; and OECD Revenue Statistics database (http://stats.oecd.org/Index.aspx?DataSetCode=REV) for lines 3, 4, and 5.

C. Tax breaks for social purposes (in millions of euros)

\begin{tabular}{|c|c|c|c|c|}
\hline & 2001 & 2003 & 2005 & $2007 *$ \\
\hline Tax breaks similar to cas $h$ benefits & 1440.0 & 2444.0 & 3855.0 & 4834.0 \\
\hline Child credits & 155.0 & 352.0 & 621 & 727 \\
\hline Combination credit (combination of work and care for children) & 263.0 & 409.0 & 754 & 955 \\
\hline Single parent credits & 472.0 & 506.0 & 618 & 687 \\
\hline Deduction for medical, disability, chronically ill or handicapped expenses, child adoption & 364.0 & 944.0 & 1712 & 2300 \\
\hline Deduction for support expenses for children & 106.0 & 136.0 & 150 & 165 \\
\hline Deduction for child care contributions & 62.0 & 97.0 & .. & .. \\
\hline Exemption for certain sign-on premiums & 18.0 & 0.0 & .. & .. \\
\hline Tax breaks to stimulate private social protection (not including pensions) & 1920.0 & 1648.0 & 680.0 & 680.0 \\
\hline Reduced wage tax for low wage employees & 890.0 & 620.0 & 186 & 186 \\
\hline Reduced wage tax for long-term unemployed & 207.0 & 130.0 & 39 & 39 \\
\hline Reduced wage tax for child care & 92.0 & 162.0 & - & - \\
\hline Reduced wage tax for paid parental leave & 18.0 & 42.0 & 51 & 51 \\
\hline Deduction of charitable and other donations & 214.0 & 231.0 & 246 & 313 \\
\hline Reduced succession duty for donations to institutions with a public interest & 117.0 & 150.0 & 158 & 158 \\
\hline Temporary additional tax credit for home help & 52.0 & 41.0 & - & - \\
\hline Tax deduction towards employment/training of workforce & 330.0 & 272.0 & .. & .. \\
\hline Reduced wage tax for schooling (non profit) & 59.0 & 72.0 &.. & .. \\
\hline Deduction for schooling (employer) & 271.0 & 200.0 & .. &.. \\
\hline \multicolumn{5}{|l|}{ Memorandum item } \\
\hline Tax breaks for pensions & .. & .. & .. & 10400.0 \\
\hline - Non-taxation of investment of private pension funds & & & & 4000.0 \\
\hline
\end{tabular}

* 2007 data in italics are Secretariat estimes.

Source: Ministry of Finance, and Ministry of Social Affairs and Employment, The Netherlands. 
DELSA/ELSA/WD/SEM(2011)9

Table Annex I.A.2.1

Detailed information on the impact of the tax system on social expenditure (cont.)

NEW ZEALAND

A. Average Itemised Tax Rates (AITR \%)

\begin{tabular}{|c|c|c|c|c|}
\hline & 2001 & 2003 & 2005 & 2007 \\
\hline Transitional Retirement Benefit & 15.9 & 16.0 & - & - \\
\hline War Veterans' allowances & 16.8 & 17.0 & 17.1 & 17.4 \\
\hline Widows Benefit & 16.3 & 16.0 & 16.1 & 16.4 \\
\hline Invalids Benefit & 16.3 & 16.3 & 16.4 & 16.6 \\
\hline Sickness benefit & 16.3 & 15.3 & 15.4 & 15.7 \\
\hline Earners account (ACC) & 7.4 & 7.0 & 6.4 & 6.3 \\
\hline Motor vehicle account (ACC) & 7.4 & 7.0 & 6.4 & 6.3 \\
\hline Non earners account (ACC) & 7.4 & 7.0 & 6.4 & 6.3 \\
\hline Medical misadventure account (ACC) & 7.4 & 7.0 & 6.4 & 6.3 \\
\hline Occupational injury: residual claims account (ACC) (formerly Employers account) & 7.4 & 7.0 & 6.4 & 6.3 \\
\hline Occupational injury: self-employed account (ACC) & 7.4 & 7.0 & 6.4 & 6.3 \\
\hline Occupational injury: employers account (ACC) & 7.4 & 7.0 & 6.4 & 6.3 \\
\hline Domestic Purposes Benefit for lone parents & 17.3 & 17.0 & 17.1 & 17.3 \\
\hline Training Benefit & 15.8 & - & - & - \\
\hline Unemployment Benefit and Emergency Unemployment Benefit & 16.5 & 15.3 & 15.4 & 15.6 \\
\hline Independent Youth Benefit & 15.5 & 15.0 & 15.0 & 15.0 \\
\hline
\end{tabular}

Source: New Zealand Treasury.

B. Average implicit indirect tax rates of consumption out of benefit income

Indirect taxes paid out of consumption of cash transfers, in millions of New Zealand dollars

\begin{tabular}{|c|c|c|c|c|c|}
\hline & & 2001 & 2003 & 2005 & 2007 \\
\hline$(1)$ & Private final consumption expenditure & 73401 & 83905 & 95498 & 105515 \\
\hline$(2)$ & Private consumption plus Government consumption minus Government wages & 84426 & 95970 & 109684 & 122381 \\
\hline \multirow[t]{3}{*}{ (3) } & General consumption taxes plus excise duties $(5110+5121)$ & 12924 & 15120 & 16438 & 16676 \\
\hline & 5110 General taxes & 10645 & 12775 & 14133 & 15046 \\
\hline & 5121 Excises & 2279 & 2345 & 2305 & 1630 \\
\hline (4) & Taxes on production sale transfer $(5100)$ & 13831 & 16135 & 17822 & 18835 \\
\hline$(5)$ & Taxes on Goods and Services (5000) & 14799 & 17259 & 19054 & 20301 \\
\hline \multicolumn{6}{|c|}{ Implicit average indirect tax rate on consumption out of benefit income: } \\
\hline (6) & using general cons umption taxes plus excise duties (3)/(2) & $15.3 \%$ & $15.8 \%$ & $15.0 \%$ & $13.6 \%$ \\
\hline (7) & using a broad concept of the indirect tax base (5)/(2) & $17.5 \%$ & $18.0 \%$ & $17.4 \%$ & $16.6 \%$ \\
\hline$(8)$ & using a broad concept of the indirect tax base and ignoring government consumpion (5)/(1) & $20.2 \%$ & $20.6 \%$ & $20.0 \%$ & $19.2 \%$ \\
\hline
\end{tabular}

Source: OECD on-line National Accounts database (http://stats.oecd.org/Index.aspx?DataSetCode=SNA_TABLE1) for lines 1 and 2; and OECD Revenue Statistics database (http://stats.oecd.org/Index.aspx?DataSetCode=REV) for lines 3, 4, and 5.

C. Tax breaks for social purposes (in millions of New Zealand dollars)

\begin{tabular}{|c|c|c|c|c|}
\hline & 2001 & 2003 & 2005 & 2007 \\
\hline Tax breaks similar to cas $h$ benefits & 23.5 & 19.7 & 20.3 & 47.0 \\
\hline Child rebate & 6.6 & 6.0 & 6.0 & 26.0 \\
\hline Child Care & 16.9 & 13.7 & 14.3 & 14.0 \\
\hline Redundancy rebate & - & - & - & 7.0 \\
\hline Tax breaks to stimulate private social protection (not including pensions) & 77.4 & 89.9 & 98.6 & 113.0 \\
\hline Charitable Donations & 77.4 & 89.9 & 98.6 & 113.0 \\
\hline
\end{tabular}

Source: New Zealand Treasury. 
DELSA/ELSA/WD/SEM(2011)9

Table Annex I.A.2.1

Detailed information on the impact of the tax system on social expenditure (cont.)

NORWAY

A. Average Itemised Tax Rates (AITR \%)

\begin{tabular}{|c|c|c|c|c|c|}
\hline & & 2001 & 2003 & 2005 & $2007 *$ \\
\hline 1 & Old-age cash benefits & 18.3 & 17.8 & 17.6 & 18.3 \\
\hline 1a & - public pensions & 16.5 & 16.0 & 15.7 & 16.6 \\
\hline $1 b$ & - early retirement benefits & 22.4 & 22.5 & 21.1 & 21.1 \\
\hline $1 \mathrm{c}$ & - private pensions & 22.1 & 21.3 & 21.3 & 21.9 \\
\hline 2 & Survivors' benefits & 19.4 & 18.9 & 18.1 & 18.7 \\
\hline $2 \mathrm{a}$ & - public pensions & 19.4 & 18.9 & 18.1 & 18.7 \\
\hline 3 & Incapacity-related benefits & 16.3 & 15.7 & 15.6 & 15.4 \\
\hline $3 a$ & - Disability pensions & 15.8 & 15.3 & 15.3 & 15.0 \\
\hline $3 \mathrm{c}$ & - Sickness payments & 25.4 & 25.0 & 26.0 & 26.1 \\
\hline 4 & Family cash benefits & 0.2 & 0.2 & 0.3 & 0.3 \\
\hline $4 c$ & - Sole parent benefits & 1.6 & 0.9 & 1.2 & 1.7 \\
\hline 5 & Active labour market policies & 17.2 & 19.8 & 19.9 & 19.4 \\
\hline $5 a$ & - benefits while on training & 17.2 & 19.8 & 19.9 & 19.4 \\
\hline 6 & Unemployment & 20.4 & 20.8 & 20.1 & 19.9 \\
\hline $6 a$ & - unemployment insurance benefit & 20.4 & 20.8 & 20.1 & 19.9 \\
\hline
\end{tabular}

Source: Ministry of Finance.

B. Average implicit indirect tax rates of consumption out of benefit income

Indirect taxes paid out of consumption of cash transfers, in millions Norwegian kroner

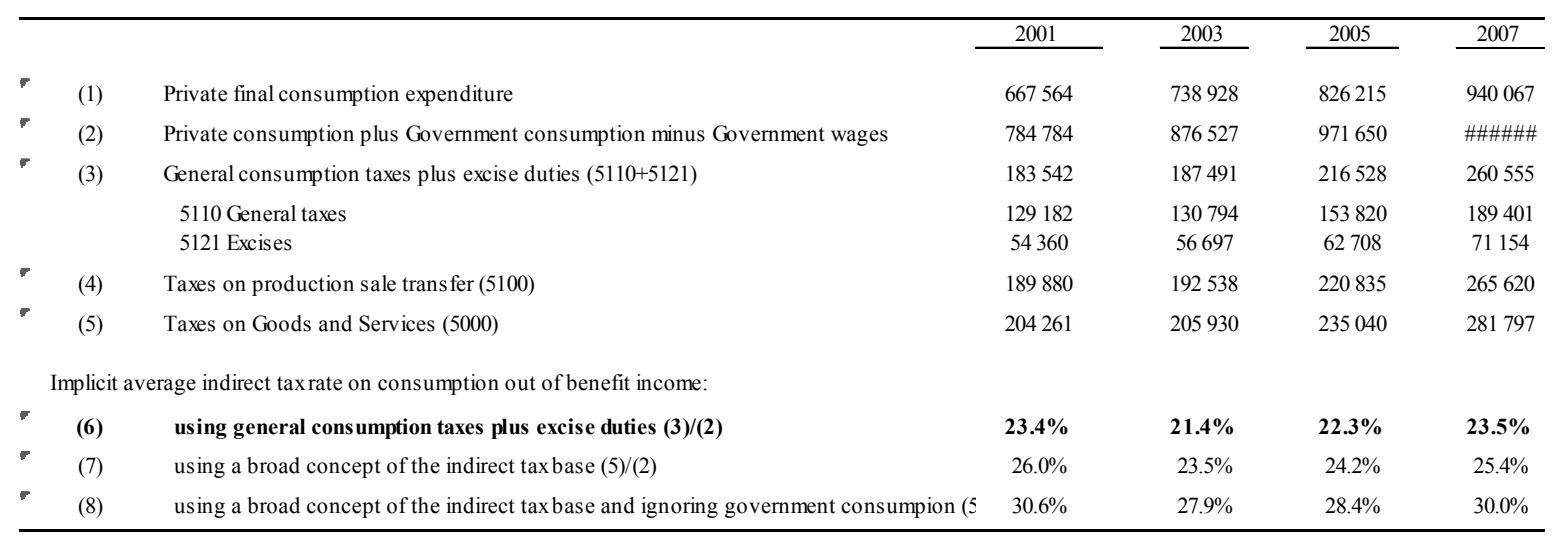

Source: OECD on-line National Accounts database (http://stats.oecd.org/Index.aspx?DataSetCode=SNA TABLE1) for lines 1 and 2; and OECD Revenue Statistics database (http://stats.oecd.org/Index.aspx?DataSetCode=REV) for lines 3, 4, and 5.

C. Tax breaks for social purposes (in millions of Norwegian kroner)

\begin{tabular}{|c|c|c|c|c|}
\hline & 2001 & 2003 & 2005 & 2007 \\
\hline Tax breaks similar to cash benefits & $\mathbf{0}$ & 2290 & 2575 & 2575 \\
\hline Childcare expense deduction & - & 1410 & 1525 & 1525 \\
\hline Healthcare expense deduction & - & 330 & 275 & 275 \\
\hline Additional personal allowance for one-parent families & - & 550 & 775 & 775 \\
\hline Tax breaks to stimulate private social protection (not including pensions) & $\mathbf{0}$ & $\mathbf{0}$ & $\mathbf{0}$ & $\mathbf{0}$ \\
\hline \multicolumn{5}{|l|}{ Memorandum item } \\
\hline Tax breaks for pensions & 11500 & 11795 & 11795 & 11795 \\
\hline Occupational pension schemes & 11500 & 11500 & 11500 & 11500 \\
\hline Individual pension schemes & - & 295 & 295 & 295 \\
\hline
\end{tabular}

* 2007 TPBSPs are estimed as in 2005.

Source: Ministry of Finance, Norway. 
DELSA/ELSA/WD/SEM(2011)9

Table Annex I.A.2.1

Detailed information on the impact of the tax system on social expenditure (cont.)

\section{POLAND}

A. Average Itemised Tax Rates (AITR \%)

\begin{tabular}{|c|c|c|}
\hline & 2005 & 2007 \\
\hline \multicolumn{3}{|l|}{ Total tax paid on transfer income (effective tax rate + effective rate of contribution) } \\
\hline old-age and disability pensions & $13.53 \% *=5.90 \%(\operatorname{tax})+7.63 \%(\mathrm{SSC})$ & $14.28 \% *=6.65 \%(\operatorname{tax})+7.63 \%(\mathrm{SSC})$ \\
\hline unemployment benefits & $13.53 \% *=5.90 \%(\operatorname{tax})+7.63 \%(\mathrm{SSC})$ & $14.28 \% *=6.65 \%(\operatorname{tax})+7.63 \%(\mathrm{SSC})$ \\
\hline sickness benefits & $16.83 \% * *(=9.40 \%(\operatorname{tax})+7.43 \%(\mathrm{SSC}))$ & $16.33 \% * *(=8.90 \%(\operatorname{tax})+7.43 \%(\mathrm{SSC})$ \\
\hline wage income & $16.83 \%=9.40 \%(\operatorname{tax})+7.43 \%(\mathrm{SSC})$ & $16.33 \%=8,90 \%(\operatorname{tax})+7,43 \%(\mathrm{SSC})$ \\
\hline
\end{tabular}

* Rate can be lower.

** On $40 \%$ of benefits only.

Source: Ministry of Finance, Poland.

B. Average implicit indirect tax rates of cons umption out of benefit income

Indirect taxes paid out of consumption of cash transfers, in millions of Polish Zloty

\begin{tabular}{|c|c|c|c|}
\hline & & 2005 & 2007 \\
\hline (1) & Private final consumption expenditure & 623360 & 711872 \\
\hline "(2) & Private consumption plus Government consumption minus Government wages & 702330 & 809734 \\
\hline \multirow[t]{2}{*}{ (3) } & General consumption taxes plus excise duties $(5110+5121)$ & 115540 & 145209 \\
\hline & $\begin{array}{l}5110 \text { General taxes } \\
5121 \text { Excises }\end{array}$ & $\begin{array}{l}74311 \\
41229\end{array}$ & $\begin{array}{l}96152 \\
49057\end{array}$ \\
\hline "(4) & Taxes on production sale transfer $(5100)$ & 117349 & 148366 \\
\hline "(5) & Taxes on Goods and Services (5000) & 124967 & 156400 \\
\hline \multicolumn{4}{|c|}{ Implicit average indirect tax rate on consumption out of benefit income: } \\
\hline '(6) & using general consumption taxes plus excise duties (3)/(2) & $16.5 \%$ & $17.9 \%$ \\
\hline (7) & using a broad concept of the indirect tax base (5)/(2) & $17.8 \%$ & $19.3 \%$ \\
\hline (8) & using a broad concept of the indirect tax base and ignoring government consumpion (5)/(1) & $20.0 \%$ & $22.0 \%$ \\
\hline
\end{tabular}

Source: OECD on-line National Accounts database (http://stats.oecd.org/Index.aspx?DataSetCode=SNA_TABLE1) for lines 1 and 2; and OECD Revenue Statistics database (http://stats.oecd.org/Index.aspx?DataSetCode=REV) for lines 3, 4, and 5 .

C. Tax breaks for social purposes (in millions of Polish Zloty)

Tax breaks similar to cash benefits

- Value of revenue foregone because of including children in the tax unit (in case of lonly parent)

- Donations to (approved) NGOs

- Donations

- Expences for rehabilitation purposes

- Children allowance

\begin{tabular}{ccc}
2005 & & 2007 \\
\cline { 3 - 3 } $\mathbf{8 5 3}$ & & $\mathbf{1 2 2 4}$ \\
398 & & 403 \\
71 & & 298 \\
47 & & 78 \\
337 & & 445 \\
- & & 532 \\
$\mathbf{0}$ & & $\mathbf{0}$ \\
& \\
& \\
$\mathbf{1 9 6 3}$ & & \\
& & \\
1963 & &..
\end{tabular}

Source: Ministry of Finance, Poland. 
DELSA/ELSA/WD/SEM(2011)9

Table Annex I.A.2.1

Detailed information on the impact of the tax system on social expenditure (cont.)

PORTUGAL

A. Amount of direct tax paid on benefit income (in millions of euros)

\begin{tabular}{lrr}
\hline & 2003 & 2005 \\
\cline { 2 - 2 } Total income tax paid on public and private transfer income & 2007 & 138.2 \\
\hline
\end{tabular}

Source: estimate by the Ministry of Finance based on Personal Income Tax Returns data.

B. Average implicit indirect tax rates of cons umption out of benefit income Indirect taxes paid out of consumption of cash transfers, in millions of euros

\begin{tabular}{|c|c|c|c|c|}
\hline & & 2003 & 2005 & 2007 \\
\hline (1) & Private final consumption expenditure & 90799 & 99846 & 110635 \\
\hline$(2)$ & Private consumption plus Government consumption minus Government wages & 99504 & 110384 & 119920 \\
\hline \multirow[t]{3}{*}{ (3) } & General consumption taxes plus excise duties $(5110+5121)$ & 16657 & 18823 & 20274 \\
\hline & 5110 General taxes & 11092 & 13006 & 14339 \\
\hline & 5121 Excises & 5565 & 5816 & 5935 \\
\hline (4) & Taxes on production sale transfer $(5100)$ & 17919 & 20212 & 21862 \\
\hline$(5)$ & Taxes on Goods and Services (5000) & 18294 & 20564 & 22284 \\
\hline \multicolumn{5}{|c|}{ Implicit average indirect tax rate on consumption out of benefit income: } \\
\hline (6) & using general cons umption taxes plus excise duties (3)/(2) & $16.7 \%$ & $17.1 \%$ & $16.9 \%$ \\
\hline (7) & using a broad concept of the indirect tax base (5)/(2) & $18.4 \%$ & $18.6 \%$ & $18.6 \%$ \\
\hline$(8)$ & using a broad concept of the indirect tax base and ignoring government consumpion (5)/(1) & $20.1 \%$ & $20.6 \%$ & $20.1 \%$ \\
\hline
\end{tabular}

Source: OECD on-line National Accounts database (http://stats.oecd.org/Index.aspx?DataSetCode=SNA_TABLE1) for lines 1 and 2; and OECD Revenue Statistics database (http://stats.oecd.org/Index.aspx?DataSetCode=REV) for lines 3, 4, and 5.

C. Tax breaks for social purposes (in millions of euros)

\section{Tax breaks similar to cash benefits}

- Health care tax credits

- Housing tax credits

- Tax credits for education expenses and for payments to homes for the elderly on behalf of taxpayers,

their relatives in the ascending line and other close relatives whose incomes do not exceed the minimum wage;

- Child tax credits

- Taxcredit for supporting relatives in the ascending line whose income does not exceed the minimumpension

- Tax benefits for disabled people

Tax breaks to stimulate private social protection (not including pensions)

- Tax credits for contributions to personal accident and life insurance

- Tax credits for contributions to health insurance

- Donations to (approved) NGOs, churches, museums, libraries, schools, research institutes and associations, and other bodies (including government bodies)

\section{Memorandum item}

Tax breaks for pensions

- Tax credits for individual retirement accounts (PPR)

- Non-taxation of investment of private pension funds

\begin{tabular}{|c|c|c|}
\hline 2003 & 2005 & 2007 \\
\hline 1438.2 & 1377.7 & 1841.1 \\
\hline 434.9 & 517.0 & 610.0 \\
\hline 395.6 & 445.0 & 503.0 \\
\hline 224.6 & 6.0 & 277.0 \\
\hline 253.2 & 265.0 & 279.0 \\
\hline 2.6 & 4.0 & 2.0 \\
\hline 127.3 & 140.7 & 170.1 \\
\hline 88.0 & 111.0 & 121.0 \\
\hline 55.1 & 67.0 & 71.0 \\
\hline 18.5 & 28.0 & 33.0 \\
\hline 14.4 & 16.0 & 17.0 \\
\hline 158.0 & 94.1 & 112.6 \\
\hline 158.0 & 94.1 & 112.6 \\
\hline .. & .. & .. \\
\hline
\end{tabular}

.. 2001 figures are not available.

Source: Portuguese Ministry of Finance, 2006, 2008 and 2009 State Budget Report ; and Portuguese Ministry of Finance, Personal Income Tax Returns data. 
DELSA/ELSA/WD/SEM(2011)9

Table Annex I.A.2.1

Detailed information on the impact of the tax system on social expenditure (cont.)

SLOVAK REPUBLIC

A. Average Itemised Tax Rates / Amount of direct tax paid on benefit income (in millions of euro)

Cash benefits paid by general government in the Slovak Republic are not subject to income tax nor to social security contributions.

B. Average implicit indirect tax rates of consumption out of benefit income

Indirect taxes paid out of consumption of cash transfers, in millions of euro

(1) Private final consumption expenditure

(2) Private consumption plus Government consumption minus Government wages

(3) General consumption taxes plus excise duties $(5110+5121)$

5110 General taxes

5121 Excises

(4) Taxes on production sale transfer (5100)

(5) Taxes on Goods and Services (5000)

\begin{tabular}{|c|c|c|c|}
\hline 2001 & 2003 & 2005 & 2007 \\
\hline 649 & 765 & 938 & 1144 \\
\hline 781 & 921 & 1119 & 1358 \\
\hline 112 & 143 & 189 & 210 \\
\hline 81 & 101 & 129 & 138 \\
\hline 31 & 42 & 60 & 72 \\
\hline 118 & 150 & 192 & 215 \\
\hline 126 & 161 & 206 & 231 \\
\hline $14.3 \%$ & $15.5 \%$ & $16.9 \%$ & $15.4 \%$ \\
\hline $16.1 \%$ & $17.5 \%$ & $18.4 \%$ & $17.0 \%$ \\
\hline $19.4 \%$ & $21.1 \%$ & $21.9 \%$ & $20.2 \%$ \\
\hline
\end{tabular}

Implicit average indirect tax rate on consumption out of benefit income:

(6) using general cons umption taxes plus excise duties (3)/(2)

(7) using a broad concept of the indirect tax base (5)/(2)

(8) using a broad concept of the indirect tax base and ignoring government consumpion (5)/(1)

$19.4 \% \quad 21.1 \%$

$17.0 \%$

Source: OECD on-line National Accounts database (http://stats.oecd.org/Index.aspx?DataSetCode=SNA_TABLE1) for lines 1 and 2; and OECD Revenue Statistics database (http://stats.oecd.org/Index.aspx?DataSetCode=REV) for lines 3, 4, and 5 .

C. Tax breaks for social purposes (in millions of euro)

Tax breaks similar to cash benefits

Child tax allowance*

Taxallowance for partially disabled people*

Taxallowance for disabled people*

* Child tax allowance and tax allowances for disabled people were deductible from tax base.

Cancelled in 2004.

Child tax credit (non wastable)

Tax breaks to stimulate private social protection (not including pensions)

Assignments to non-profit sector for selected purposes*:

- assignments given by individuals

-assignments given by legal entities - social and health purposes

* Each taxpayer can assign $2 \%$ from his tax liability ( $1 \%$ in 2003$)$, limits for assignments:

taxpayer - individual - minimum 20 SKK, taxpayer - legal entity - minimum 250 SKK

Donations to municipatlities and legal entities for selected purposes**:

- donations given by individuals

- donations given by legal entities - social and health purposes

** Tax deductibility limits for donations: taxpayer - individual - minimum 500 SKK and the

value of donation can not exceed $10 \%$ of tax base, taxpayer - legal entity - minimum 2000 SKK and the value of donation can not exceed $2 \%$ of tax base. Cancelled in 2004.

Reduction of tax for employers hiring disabled people***:

- taxpayers who filed tax return for personal income tax purposes

taxpayers who filed tax return for corporate income tax purposes

*** Reduction of tax (tax relief) for employers hiring disabled people: $10000 \mathrm{SKK} / \mathrm{employee}$ or

24000 SKK/employee per year according to the level of dis ability. Cancelled in 2004.

\section{Memorendum iten}

\section{Tax breaks for pensions}

- Deduction of contributions to private pensions (e.g occupational pension plans, individual retirement accounts, RRSPs, Superannuation, etc) $* * * *$

**** In 2005 a mandatory fully funded pillar (privately managed) of the pension system has been introduced. Part of social security contributions ( 9 percentage points) is accumulated in private pension funds and these amounts are non-taxable. As all mandatory social security contributions are non taxable there are not considered as tax breaks.

\begin{tabular}{|c|c|c|c|}
\hline 2001 & 2003 & 2005 & 2007 \\
\hline 109.7 & 210.8 & 0.0 & 0.0 \\
\hline 100.9 & 200.5 & - & - \\
\hline 2.4 & 4.1 & - & - \\
\hline 6.4 & 6.2 & - & - \\
\hline - & - & $\mathrm{x}$ & $\mathrm{x}$ \\
\hline 22.6 & 54.4 & 30.9 & 42.1 \\
\hline - & 3.2 & 10.4 & 12.8 \\
\hline - & - & 20.5 & 29.3 \\
\hline 10.0 & 10.9 & - & - \\
\hline 6 & 37.6 & & \\
\hline
\end{tabular}

Source: Data provided by Ministry of Labour, Social Affairs and Family of the Slovak Republic - aggregate data from filed tax returns (personal income tax and corporate income tax) in 2001, 2003 and by Ministry of Finance of the Slovak Republic in 2005 and 2007. 
DELSA/ELSA/WD/SEM(2011)9

Table Annex I.A.2.1

Detailed information on the impact of the tax system on social expenditure (cont.)

SPAIN

A. Amount of direct tax paid on benefit income (in millions of euros)

\begin{tabular}{|c|c|c|c|c|}
\hline & 2001 & $2003 *$ & 2005 & 2007 \\
\hline $\begin{array}{l}\text { Total tax paid (including social security contribution) on public trans fer income } \\
\text { of which: }\end{array}$ & 7127 & 7805 & 9088 & 11785 \\
\hline - Income tax* & 6424 & 7433 & 8808 & 10666 \\
\hline - Social security contributions $* *$ & 703 & 372 & 280 & 1119 \\
\hline $\begin{array}{l}\text { Total tax paid (including social security contribution) on private transfer income } \\
\text { of which: }\end{array}$ & 1321 & 1646 & 1739 & 2115 \\
\hline - Income tax * & 1321 & 1646 & 1739 & 2115 \\
\hline Total tax paid (including social security contribution) on transfer income & 8448 & 9451 & 10827 & 13900 \\
\hline
\end{tabular}

* 2003 estimates based on 2002 PIT statistics. Data are based on income class data based on data-set of individual taxpayers. The estimation method is based on calculations made on personal income tax payments multiplied by fractions of net taxable income sources (as percentage of the total tax base) at the level of income classes/tax brackets.

** SSC paid by unemployed workers perceiving unemployment benefits.

Sources: Tax Expenditures Budget, Ministry of Finance and Ministry of Labor and Immigration, Spain.

B. Average implicit indirect tax rates of cons umption out of benefit income Indirect taxes paid out of consumption of cash trans fers, in millions of euros

\begin{tabular}{|c|c|c|c|c|c|}
\hline & & 2001 & 2003 & 2005 & 2007 \\
\hline$(1)$ & Private final consumption expenditure & 402294 & 451208 & 525124 & 604022 \\
\hline$(2)$ & Private consumption plus Government consumption minus Government wages & 449756 & 508436 & 597853 & 689661 \\
\hline (3) & General consumption taxes plus excise duties $(5110+5121)$ & 55908 & 65019 & 77250 & 86147 \\
\hline & $\begin{array}{l}5110 \text { General taxes } \\
5121 \text { Excises }\end{array}$ & $\begin{array}{l}39226 \\
16682\end{array}$ & $\begin{array}{l}45897 \\
19122\end{array}$ & $\begin{array}{l}56396 \\
20854\end{array}$ & $\begin{array}{l}63400 \\
22748\end{array}$ \\
\hline$(4)$ & Taxes on production sale transfer $(5100)$ & 60664 & 70315 & 83408 & 92785 \\
\hline$(5)$ & Taxes on Goods and Services (5000) & 66239 & 76059 & 89964 & 99653 \\
\hline \multicolumn{6}{|c|}{ Implicit average indirect tax rate on consumption out of benefit income: } \\
\hline (6) & using general consumption taxes plus excise duties (3)/(2) & $12.4 \%$ & $12.8 \%$ & $12.9 \%$ & $12.5 \%$ \\
\hline (7) & using a broad concept of the indirect tax base (5)/(2) & $14.7 \%$ & $15.0 \%$ & $15.0 \%$ & $14.4 \%$ \\
\hline$(8)$ & using a broad concept of the indirect tax base and ignoring government consumpion (5)/(1) & $16.5 \%$ & $16.9 \%$ & $17.1 \%$ & $16.5 \%$ \\
\hline
\end{tabular}

Source: OECD on-line National Accounts database (http://stats.oecd.org/Index.aspx?DataSetCode=SNA_TABLE1) for lines 1 and 2; and OECD Revenue Statistics database (http://stats.oecd.org/Index.aspx?DataSetCode=REV) for lines 3, 4, and 5.

C. Tax breaks for social purposes (in millions of euros)

\begin{tabular}{|c|c|c|c|c|}
\hline & 2001 & 2003 & 2005 & 2007 \\
\hline Tax breaks similar to cash benefits & 238 & 1064 & 1416 & 3373 \\
\hline PIT exemption of Invalidity, Terrorism and HIV Pensions & 238 & 289 & 329 & 419 \\
\hline Child Care Benefit exemption & - & 61 & 154 & 198 \\
\hline Child Tax Credit & - & 583 & 739 & 2046 \\
\hline Unemployment Lump-Sum Payment \& Cease Job Compensation & - & 132 & 161 & 174 \\
\hline Labour extension \& Labour mobility & - & - & 33 & 52 \\
\hline Disability of active workers & - & - & - & 245 \\
\hline Personal Allowance for one-parent families & - & - & - & 240 \\
\hline Tax breaks to stimulate private social protection (not including pensions) & $\mathbf{0}$ & 2185 & 2806 & 3237 \\
\hline Reduction of SSC for employers hiring disadvantaged groups (long-term unemployed, disabled $(* *)$ & - & 2185 & 2806 & 3237 \\
\hline \multicolumn{5}{|l|}{ Memorandum item } \\
\hline Tax breaks for pensions & 1208 & 1862 & 2408 & 2420 \\
\hline
\end{tabular}

Sources: Tax Expenditures Budget, Ministry of Finance, Spain. 
DELSA/ELSA/WD/SEM(2011)9

Table Annex I.A.2.1

Detailed information on the impact of the tax system on social expenditure (cont.)

SWEDEN

A. Average Itemis ed Tax Rates (AITR \%)

\begin{tabular}{|c|c|c|c|c|}
\hline & 2001 & 2003 & 2005 & 2007 \\
\hline \multicolumn{5}{|l|}{ 1. Old-age cash benefits } \\
\hline a - public pensions & 25.0 & 28.6 & 28.8 & 28.1 \\
\hline $\mathrm{b}$ - early retirement pensions & 29.0 & 28.6 & 28.8 & 28.1 \\
\hline $\mathrm{c}$ - private pensions & 32.1 & 28.6 & 28.8 & 28.1 \\
\hline \multicolumn{5}{|l|}{ 2. Survivors benefits } \\
\hline a - public pensions & 22.3 & 28.3 & 28.5 & 27.1 \\
\hline \multicolumn{5}{|l|}{$\mathrm{b}$ - private pensions } \\
\hline \multicolumn{5}{|l|}{ 3. Incapacity-related benefits } \\
\hline a - disability pensions & 24.8 & 27.7 & 28.1 & 26.7 \\
\hline b - occupational injury benefits & 32.4 & 30.8 & 30.6 & 27.5 \\
\hline c-sickness payments & 34.1 & 30.8 & 30.6 & 27.5 \\
\hline \multicolumn{5}{|l|}{ 4. Family cash benefits } \\
\hline a - family benefits & 12.9 & 12.9 & 13.6 & 12.8 \\
\hline b - maternity and parental leave payments & 33.8 & 30.8 & 30.6 & 27.5 \\
\hline \multicolumn{5}{|l|}{ c-sole parent benefits } \\
\hline \multicolumn{5}{|l|}{ 5. Active labor market policies } \\
\hline $\mathrm{a}$ - benefits while in training & 29.6 & 0.0 & 0.0 & 0.0 \\
\hline \multicolumn{5}{|l|}{ 6. Unemployment } \\
\hline a - unemployment insurance benefit & 29.8 & 28.7 & 28.0 & 25.1 \\
\hline
\end{tabular}

Source: Statistics Sweden - Social Protection Expenditure and Receipts in Sweden and Europe 2002-2007 http://www.scb.se/NR0201-EN

B. Average implicit indirect tax rates of cons umption out of benefit income

Indirect taxes paid out of consumption of cash transfers, in millions of Swedish kronor

\begin{tabular}{|c|c|c|c|c|c|}
\hline & & 2001 & 2003 & 2005 & 2007 \\
\hline$(1)$ & Private final consumption expenditure & 1145837 & 1241140 & 1336052 & 1460162 \\
\hline$(2)$ & Private consumption plus Government consumption minus Government wages & 1397867 & 1526984 & 1629743 & 1791632 \\
\hline \multirow[t]{2}{*}{ (3) } & General consumption taxes plus excise duties $(5110+5121)$ & 280075 & 306868 & 333060 & 371028 \\
\hline & $\begin{array}{l}5110 \text { General taxes } \\
5121 \text { Excises }\end{array}$ & $\begin{array}{c}206544 \\
73531\end{array}$ & $\begin{array}{l}226380 \\
80488\end{array}$ & $\begin{array}{c}250470 \\
82590\end{array}$ & $\begin{array}{c}286211 \\
84818\end{array}$ \\
\hline$(4)$ & Taxes on production sale transfer $(5100)$ & 288473 & 315650 & 342350 & 380990 \\
\hline$(5)$ & Taxes on Goods and Services (5000) & 296136 & 323978 & 353370 & 394073 \\
\hline \multicolumn{6}{|c|}{ Implicit average indirect tax rate on consumption out of benefit income: } \\
\hline$(6)$ & using general consumption taxes plus excise duties (3)/(2) & $20.0 \%$ & $20.1 \%$ & $20.4 \%$ & $20.7 \%$ \\
\hline$(7)$ & using a broad concept of the indirect tax base (5)/(2) & $21.2 \%$ & $21.2 \%$ & $21.7 \%$ & $22.0 \%$ \\
\hline$(8)$ & using a broad concept of the indirect tax base and ignoring government consumpion (5)/(1) & $25.8 \%$ & $26.1 \%$ & $26.4 \%$ & $27.0 \%$ \\
\hline
\end{tabular}

Source: OECD on-line National Accounts database (http://stats.oecd.org/Index.aspx?DataSetCode=SNA_TABLE1) for lines 1 and 2; and OECD Revenue Statistics database (http://stats.oecd.org/Index.aspx?DataSetCode=REV) for lines 3, 4, and 5.

\section{Tax breaks for social purposes (in millions of Swedish kronor)}

Information on TBSPs that were not accounted in the direct tax calculations is not available. 
Table Annex I.A.2.1

Detailed information on the impact of the tax system on social expenditure (cont.)

\section{TURKEY}

A. Amount of direct tax paid on benefit income (in millions of of New Turkish liras (TRY))

Social benefits are not subject to taxation (according to the Article 25 of the Law Number 193 (which is PIT Law).

B. Average implicit indirect tax rates of cons umption out of benefit income

Indirect taxes paid out of consumption of cash transfers, in millions of pesos)

\begin{tabular}{|c|c|c|c|}
\hline & & 2005 & 2007 \\
\hline$(1)$ & Private final consumption expenditure & 465402 & 601239 \\
\hline$(2)$ & Private consumption plus Government consumption minus Government wages & 541900 & 709055 \\
\hline \multirow[t]{3}{*}{ (3) } & General consumption taxes plus excise duties $(5110+5121)$ & 67685 & 82396 \\
\hline & 5110 General taxes & 34357 & 43285 \\
\hline & 5121 Excises & 33328 & 39111 \\
\hline$(4)$ & Taxes on production sale transfer $(5100)$ & 74561 & 92605 \\
\hline$(5)$ & Taxes on Goods and Services (5000) & 77605 & 96766 \\
\hline \multicolumn{4}{|c|}{ Implicit average indirect tax rate on consumption out of benefit income: } \\
\hline (6) & using general consumption taxes plus excise duties (3)/(2) & $12.5 \%$ & $11.6 \%$ \\
\hline (7) & using a broad concept of the indirect tax base (5)/(2) & $0.0 \%$ & $0.0 \%$ \\
\hline$(8)$ & using a broad concept of the indirect tax base and ignoring government consumpion $(5) /(1)$ & $0.0 \%$ & $0.0 \%$ \\
\hline
\end{tabular}

Source: OECD on-line National Accounts database (http://stats.oecd.org/Index.aspx?DataSetCode=SNA_TABLE1) for lines 1 and 2; and OECD Revenue Statistics database (http://stats.oecd.org/Index.aspx?DataSetCode=REV) for lines 3, 4, and 5.

C. Tax breaks for social purposes (in millions of pesos)

\begin{tabular}{|c|c|c|}
\hline & 2005 & 2007 \\
\hline Tax breaks Similar to cash benefits & 0.0 & 0.0 \\
\hline Tax breaks to stimulate private social protection (not including pensions) & $\mathbf{0 . 0}$ & $\mathbf{0 . 0}$ \\
\hline \multicolumn{3}{|l|}{ Memorendum item } \\
\hline Tax breaks to pensions & 0.0 & 0.0 \\
\hline
\end{tabular}

Source: Ministry of Finance. 
DELSA/ELSA/WD/SEM(2011)9

Table Annex I.A.2.1

Detailed information on the impact of the tax system on social expenditure (cont.)

\section{UNITED KINGDOM}

A. Average Itemised Tax Rates (AITR \%)

\begin{tabular}{|c|c|c|c|c|c|}
\hline & & 2001 & 2003 & 2005 & 2007 \\
\hline 1 & Old-age cash benefits & & & & \\
\hline & - public pensions & 3.00 & 3.26 & 4.15 & 4.45 \\
\hline $1 \mathrm{c}$ & - private pensions & 7.00 & 6.97 & 14.08 & 13.48 \\
\hline 2 & Survivors' benefits & & & & \\
\hline & - public pensions & 8.00 & 9.69 & 10.23 & 10.04 \\
\hline & - Widows Pension & 9.00 & 9.69 & 10.23 & 10.04 \\
\hline & - War Widows Pension & 0.00 & 0.00 & 0.00 & 0.00 \\
\hline 3 & Incapacity-related benefits & & & & \\
\hline $3 a$ & - Disability pensions & 0.00 & 0.00 & 0.00 & 0.00 \\
\hline $3 b$ & - Occupational Injury benefits & 0.00 & 0.00 & 0.00 & 0.00 \\
\hline $3 c$ & - Sickness payments & 10.00 & 10.00 & 13.38 & 14.93 \\
\hline & - Statutory sick pay & 14.00 & 15.25 & 14.74 & 12.89 \\
\hline & - Inacapacity Benefit: Short-term & 0.00 & 0.00 & 0.00 & 0.00 \\
\hline & - Inacapacity Benefit: Long-term & 2.00 & 2.82 & 13.28 & 15.04 \\
\hline $3 d$ & - Disability Allowances & 0.00 & 0.00 & 0.00 & 0.00 \\
\hline 4 & Family cash benefits & & & & \\
\hline $4 \mathrm{a}$ & - Family benefits & 0.00 & 0.00 & 0.00 & 0.00 \\
\hline $4 \mathrm{~b}$ & - Maternity and parental leave payments & 11.00 & 14.54 & 17.59 & 14.48 \\
\hline & - Maternity Allowance & 0.00 & 0.00 & 0.00 & 0.00 \\
\hline & - Statutory Maternity Allowance & 12.00 & 14.54 & 17.59 & 14.48 \\
\hline $4 \mathrm{c}$ & - Sole parent benefits & 0.00 & 0.00 & 0.00 & 0.00 \\
\hline $4 d$ & - Child Benefit & 0.00 & 0.00 & 0.00 & 0.00 \\
\hline 5 & Active labour market policies & & & & \\
\hline $5 \mathrm{a}$ & - benefits while on training & 0.00 & 0.00 & 0.00 & 0.00 \\
\hline 6 & Unemployment & & & & \\
\hline $6 \mathrm{~b}$ & - unemployment assistance benefit & 1.00 & 0.82 & 0.12 & 0.06 \\
\hline 7 & Housing & & & & \\
\hline $7 \mathrm{a}$ & - rent subsidies & 0.00 & 0.00 & 0.00 & 0.00 \\
\hline 9 & Wage income & 18.0 & 18.99 & 25.07 & 25.69 \\
\hline
\end{tabular}

Source: IGOTM Tax Benefit Model based on the (2001-02, 2003-04, 2005-06 and 2007-08) Family Resource Survey.

B. Average implicit indirect tax rates of cons umption out of benefit income

Indirect taxes paid out of consumption of cash transfers, in millions of pounds sterling

(1) Private final consumption expenditure

\begin{tabular}{|c|c|c|c|}
\hline 2001 & 2003 & 2005 & 2007 \\
\hline 672889 & 742276 & 814964 & 892990 \\
\hline 764548 & 853617 & 941450 & 1034132 \\
\hline 103648 & 115389 & 122675 & 132656 \\
\hline 67051 & 77308 & 83434 & 92043 \\
\hline 36597 & 38081 & 39241 & 40613 \\
\hline 110769 & 122847 & 130316 & 141358 \\
\hline 115324 & 128013 & 135552 & 147324 \\
\hline $13.6 \%$ & $13.5 \%$ & $13.0 \%$ & $12.8 \%$ \\
\hline $15.1 \%$ & $15.0 \%$ & $14.4 \%$ & $14.2 \%$ \\
\hline $17.1 \%$ & $17.2 \%$ & $16.6 \%$ & $16.5 \%$ \\
\hline
\end{tabular}

(2) Private consumption plus Government consumption minus Government wages

(3) General consumption taxes plus excise duties $(5110+5121)$

$$
\begin{aligned}
& 5110 \text { General taxes } \\
& 5121 \text { Excises }
\end{aligned}
$$

(4) Taxes on production sale transfer (5100)

(5) Taxes on Goods and Services (5000)

Implicit average indirect tax rate on consumption out of benefit income:

(6) using general cons umption taxes plus excise duties (3)/(2)

(7) using a broad concept of the indirect tax base (5)/(2)

$17.2 \%$

$16.5 \%$

(8) using a broad concept of the indirect tax base and ignoring government consumpion (5)/(1)

Source: OECD on-line National Accounts database (http://stats.oecd.org/Index.aspx?DataSetCode=SNA TABLE1) for lines 1 and 2; and OECD Revenue Statistics database (http://stats.oecd.org/Index.aspx?DataSetCode=REV) for lines 3, 4, and 5 . 
DELSA/ELSA/WD/SEM(2011)9

Table Annex I.A.2.1

Detailed information on the impact of the tax system on social expenditure (cont.)

\section{UNITED KINGDOM}

C. Tax breaks for social purposes (in millions of pounds sterling)

\begin{tabular}{|c|c|c|c|c|}
\hline & 2001 & 2003 & 2005 & 2007 \\
\hline Tax breaks similar to cas $h$ benefits & 793.0 & 4010.0 & 4400.0 & 4700.0 \\
\hline \multicolumn{5}{|l|}{ Family } \\
\hline Working families'Tax Credit (negative tax) & 758.0 & - & - & \\
\hline $\operatorname{tax}$ & 758.0 & & & \\
\hline cash & 4742.0 & & & \\
\hline total & 5500.0 & & & \\
\hline Working Tax Credit ( negative tax ) & & 1200.0 & 1000.0 & 1000.0 \\
\hline $\operatorname{tax}$ & & 1200.0 & 1000.0 & 1000.0 \\
\hline cash & & 3500.0 & 3700.0 & 3700.0 \\
\hline total & & 4700.0 & 4800.0 & 4700.0 \\
\hline Child Tax Credit (negative tax) & & 2800.0 & 3400.0 & 3700.0 \\
\hline $\operatorname{tax}$ & & 2800.0 & 3400.0 & 3700.0 \\
\hline cash & & 6000.0 & 9200.0 & 11700.0 \\
\hline total & & 8800.0 & 12600.0 & 15400.0 \\
\hline \multicolumn{5}{|l|}{ Other Income Maintenance } \\
\hline Exemption of the first 30000 pounds of severance payments & .. & .. & .. & .. \\
\hline Additional personal allowance for one parent families & .. & .. & .. & .. \\
\hline Charitable donations under the payroll giving scheme & 25.0 & .. & .. & .. \\
\hline Outplacement couns elling for redundant employees & 10.0 & 10.0 & .. & .. \\
\hline Tax breaks to stimulate private social protection (not including pensions) & 1200.0 & 1260.0 & 1560.0 & 1680.0 \\
\hline \multicolumn{5}{|l|}{ Health } \\
\hline Insurance premiums and medical care (abolished in 1999) & 0.0 & 0.0 & 0.0 & 0.0 \\
\hline \multicolumn{5}{|l|}{ Other } \\
\hline Income of charities & 860.0 & 920.0 & 1100.0 & 1300.0 \\
\hline Exemption to charities on death & 340.0 & 340.0 & 460.0 & 380.0 \\
\hline \multicolumn{5}{|l|}{ Memorandum item } \\
\hline Tax breaks for pensions & 9400.0 & 11700.0 & 15400.0 & .. \\
\hline Total reliefs & 17000.0 & 20100.0 & 25000.0 & \\
\hline Deduction of contributions to private pensions by employees and self-employed & 5550.0 & 5800.0 & 6600.0 & \\
\hline Deduction of contributions to private pensions by employers & 7400.0 & 10200.0 & 13600.0 & \\
\hline Non-taxation of investment of private pension funds & 3700.0 & 3800.0 & 4500.0 & \\
\hline Relief on lump sumpayments from unfunded schemes & 350.0 & 300.0 & 300.0 & \\
\hline - taxation of current pensions in payment & 7600.0 & 8400.0 & 9600.0 & \\
\hline
\end{tabular}

Sources: Estimates based on administrative data and information compiled from a variety of sources by the Office for National Statistics, for HM Revenue and Customs. 
DELSA/ELSA/WD/SEM(2011)9

Table Annex I.A.2.1

Detailed information on the impact of the tax system on social expenditure (cont.)

\section{UNITED STATES}

A. Average Itemis ed Tax Rates (AITR \%)

\begin{tabular}{|c|c|c|c|c|}
\hline & 2001 & 2003 & 2005 & 2007 \\
\hline Social Security Benefits & 4.0 & 3.6 & 3.8 & 4.6 \\
\hline Unemployment compensation & 7.7 & 5.7 & 5.6 & 6.0 \\
\hline Pension and IRA distributions & 14.5 & 11.7 & 12.4 & 12.9 \\
\hline
\end{tabular}

Source: US Department of Treasury.

The AITRs for wage income, superannuation pensions and superannuation lump sums were calculated using a sample file of

The AITRs were obtained by calculating the amount of tax paid in aggregate with and without the income streams. The difference

Sources: STINMOD distributional model. Revenue Group of The Treasury, Australian Government.

B. Average implicit indirect tax rates of cons umption out of benefit income

Indirect taxes paid out of consumption of cash transfers, in millions US dollars

(1) Private final consumption expenditure

\begin{tabular}{|c|c|c|c|}
\hline 2001 & 2003 & 2005 & 2007 \\
\hline \#\#\#\#\#\#\# & 7804000 & 8819000 & 9826400 \\
\hline \#\#\#\#\#\#\# & 8406800 & 9526400 & 10638800 \\
\hline \#\#\#\#\#\#\# & 355799 & 404802 & 437366 \\
\hline \#\#\#\#\#\#\# & 236046 & 273898 & 300061 \\
\hline \#\#\#\#\#\#\#\# & 119753 & 130904 & 137305 \\
\hline \#\#\#\#\#\#\# & 437365 & 498282 & 534202 \\
\hline \#\#\#\#\#\#\#\# & 515606 & 599547 & 644821 \\
\hline $4.4 \%$ & $4.2 \%$ & $4.2 \%$ & $4.1 \%$ \\
\hline $6.2 \%$ & $6.1 \%$ & $6.3 \%$ & $6.1 \%$ \\
\hline $6.6 \%$ & $6.6 \%$ & $6.8 \%$ & $6.6 \%$ \\
\hline
\end{tabular}

(2) Private consumption plus Government consumption minus Government wages

(3) General consumption taxes plus excise duties $(5110+5121)$ 5110 General taxes

5121 Excises

(4) Taxes on production sale transfer (5100)

(5) Taxes on Goods and Services (5000)

Implicit average indirect tax rate on consumption out of benefit income:

\begin{tabular}{|c|c|c|c|c|}
\hline (6) using general cons umption taxes plus excise duties (3)/(2) & $4.4 \%$ & $4.2 \%$ & $4.2 \%$ & $4.1 \%$ \\
\hline (7) using a broad concept of the indirect tax base (5)/(2) & $6.2 \%$ & $6.1 \%$ & $6.3 \%$ & $6.1 \%$ \\
\hline (8) using a broad concept of the indirect tax base and ignoring government consumpion (5)/(1) & $6.6 \%$ & $6.6 \%$ & $6.8 \%$ & $6.6 \%$ \\
\hline
\end{tabular}

Source: OECD on-line National Accounts database (http://stats.oecd.org/Indexaspx?DataSetCode=SNA TABLE1) for lines 1 and 2; and OECD Revenue Statistics database (http://stats.oecd.org/Index.aspx?DataSetCode=REV) for lines 3, 4, and 5. 
DELSA/ELSA/WD/SEM(2011)9

Table Annex I.A.2.1

Detailed information on the impact of the tax system on social expenditure (cont.)

\section{UNITED STATES}

C. Tax breaks for social purposes (in millions of US dollars)

Tax breaks similar to cas $h$ benefits

\begin{tabular}{|c|c|c|c|}
\hline 2001 & 2003 & 2005 & 2007 \\
\hline 78658 & 84304 & 91916 & 83830 \\
\hline 4990 & 6240 & 6110 & 4470 \\
\hline 20 & -30 & 1050 & 760 \\
\hline 41 & 40 & 40 & 30 \\
\hline 4940 & 5089 & 4925 & 4990 \\
\hline 3182 & 3310 & 3680 & 3950 \\
\hline 500 & 430 & 440 & 420 \\
\hline 130 & 220 & 360 & 370 \\
\hline 190 & 250 & 310 & 350 \\
\hline 29312 & 37970 & 41790 & 30910 \\
\hline 35353 & 30785 & 33211 & 37580 \\
\hline 116470 & 141320 & 159610 & 186490 \\
\hline 82800 & 101920 & 118420 & 133790 \\
\hline 1520 & 2550 & 3790 & 4260 \\
\hline - & - & - & - \\
\hline 270 & 3390 & 3350 & 4310 \\
\hline 140 & 350 & 710 & 620 \\
\hline 50 & 160 & 210 & 260 \\
\hline 50 & 50 & 30 & 30 \\
\hline 30150 & 30020 & 29670 & 38200 \\
\hline 380 & 1070 & 1120 & 1450 \\
\hline 10 & 190 & 430 & 810 \\
\hline 1100 & 1620 & 1880 & 2760 \\
\hline
\end{tabular}

Deductibility of medical expenses

Medical savings accounts

Additional deduction for the blind

Earned income credit

Credit for child and dependent care expenses \& exclusion for employer provided child care

Exclusion. of certain foster care payments

Adoption assistance (adoption credit and exclusion)

Assistance for adopted foster children

Child credit (from 1998 onwards)

Personal allowance for dependants (largely for children)

Tax breaks to stimulate private social protection (not including pensions)

Exclusion. of employer contributions for medical insurance premiums and medical care

Self-employed medical insurance premiums

Exclusion. of interest on State and local debt for private non-profit health facilities (excl. interest hospital construction bonds)

Deductibility of charitable contributions (health)

Special Blue Cross/Blue Shield deduction

Tax credit for orphan drug research

Credit for disabled access expenditures

Deductibility of charitable contributions, other than education or health

Empowerment zones, enterprise communities, renewal communities

New markets tax credit

Exclusion of hospital construction bonds

$\begin{array}{cccc}110990 & \mathbf{1 3 8 ~ 0 0 0} & \mathbf{1 0 1 8 8 0} & \mathbf{1 1 4 3 2 0} \\ 42070 & 59480 & 50630 & 47060 \\ 44080 & 51560 & 37440 & 46000 \\ 18680 & 20060 & 3100 & 9500 \\ - & 880 & 1310 & 760 \\ 6160 & 6020 & 9400 & 11000\end{array}$

\section{Memorandum item}

Tax breaks for pension *

Net exclusion of pension contributions:

Employer plans

401(K) plans

Individual retirement accounts

Low and moderate income savers credit

Keogh plans

6160

6020

9400

11000

Small business retirment credit

* Estimates for later years reflect a change in the baseline. Lower tax rates on dividends and capital gains on corporate equity are not considered tax preferences.

Sources: Office of Management and Budget, Analytical Perspectives, Budget of the United States Government and US Department of Treasury. 


\section{A.I.2.2. Net Social Expenditure Indicators Related to GDP at Market Prices and National Income, 2007}

As the construction of net social spending indicators involves adjusting for indirect taxation of consumption out of benefit income, net social expenditure is related to GDP at factor cost, as GDP at factor costs does not include the value of indirect taxation and government subsidies to private enterprises and public corporations. However, in order to facilitate comparison with gross social spending indicators which are usually related to GDP at market prices for international comparisons, Table A.I.2.2a presents these indicators. As domestic product includes income that accrues to foreigners, it may be argued that national income is another appropriate measure. As net transfers to foreigners should be measured (foreign aid is often net of tax) and capital stock depreciation arguably should not be used to finance tax payments, Table A.I.2.2b relates the net spending indicators to net disposable national income at factor prices. 
Table A.I.2.2a: From gross public to total net social spending, 2007

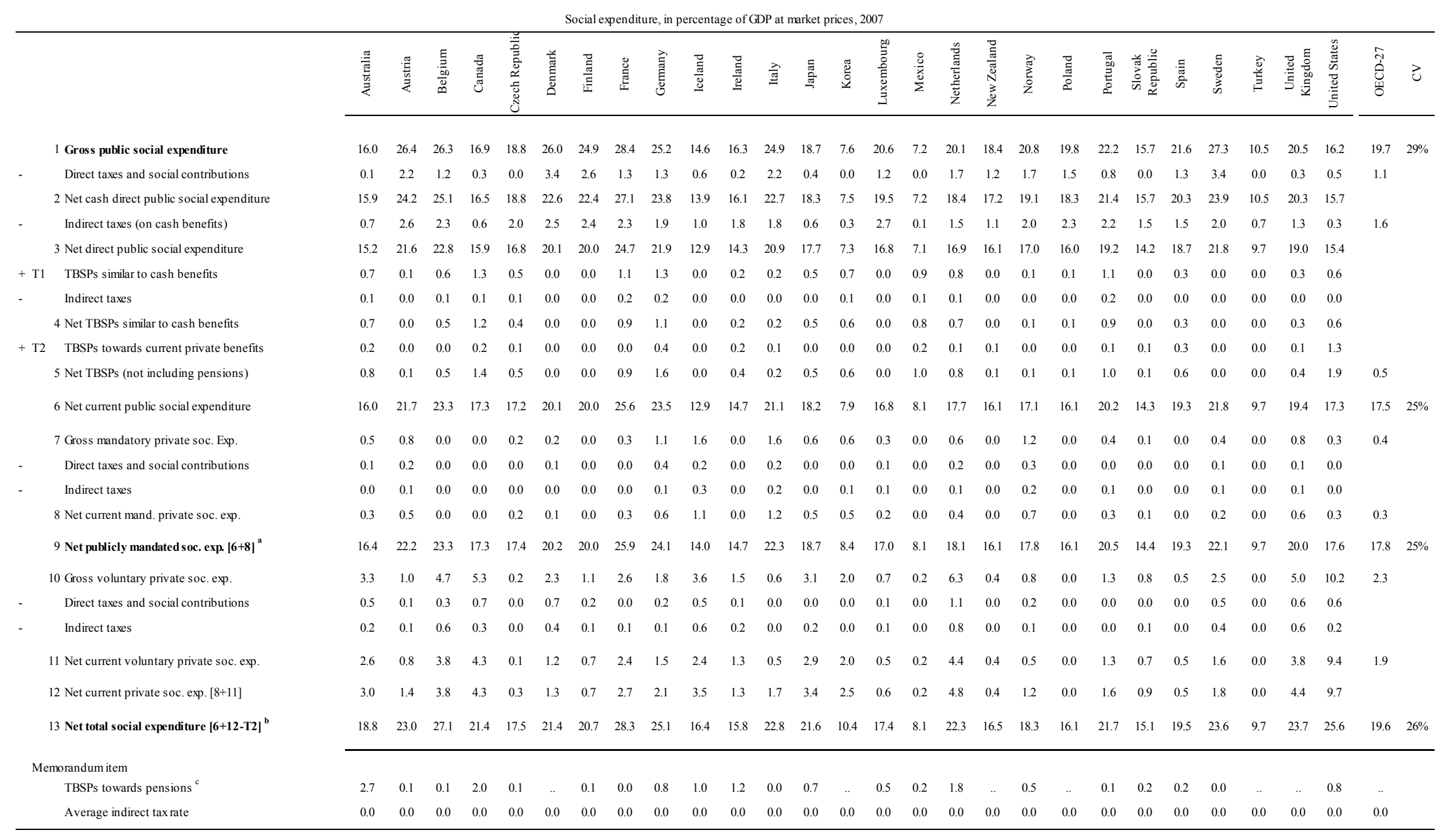

a) Numbers in square brackets refer to line numbers in the second column; ".." cell with no information.

b) In order to avoid double counting, the value of TBSPs towards "current" private social benefits has been ignored for the calculation of net total social expenditure.

c) Because of conceptual issues and gaps in data availability, tax breaks towards old-age pensions are shown in the table as a memorandum item. 
Table A.I.2.2b: From gross public to total net social spending, 2007

\begin{tabular}{|c|c|c|c|c|c|c|c|c|c|c|c|c|c|c|c|c|c|c|c|c|c|c|c|c|c|c|c|c|}
\hline \multicolumn{29}{|c|}{ Social expenditure, in percentage of NNI, 2007} \\
\hline & 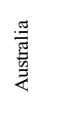 & 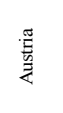 & 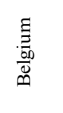 & $\begin{array}{l}\text { 䔍 } \\
\text { 嵌 }\end{array}$ & 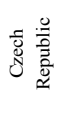 & 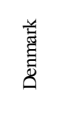 & 惫 & 总 & 产 & 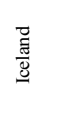 & 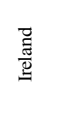 & 忞 & 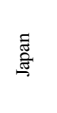 & 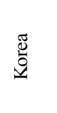 & 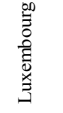 & $\frac{8}{\frac{8}{8}}$ & 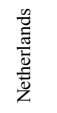 & 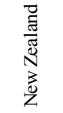 & 离 & 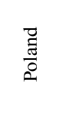 & 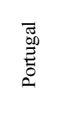 & 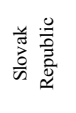 & 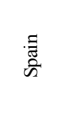 & 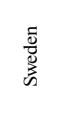 & $\begin{array}{l}\text { 总 } \\
\text { 言 } \\
\text { 总 }\end{array}$ & 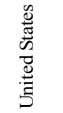 & 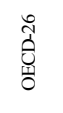 & z \\
\hline 1 Gross public social expenditure & 19.9 & 31.6 & 31.0 & 19.6 & 24.8 & 30.6 & 29.4 & 32.3 & 28.9 & 17.9 & 21.1 & 29.7 & 23.0 & 8.7 & 29.6 & 8.0 & 23.0 & 23.4 & 24.0 & 23.5 & 27.8 & 19.5 & 26.3 & 30.4 & 22.7 & 18.2 & 24.0 & $27 \%$ \\
\hline Direct taxes and social contributions & 0.2 & 2.7 & 1.5 & 0.4 & 0.0 & 4.1 & 3.0 & 1.5 & 1.5 & 0.8 & 0.3 & 2.6 & 0.5 & 0.0 & 1.7 & 0.0 & 2.0 & 1.5 & 2.0 & 1.7 & 1.0 & 0.0 & 1.6 & 3.8 & 0.3 & 0.6 & 1.4 & \\
\hline 2 Net cash direct public social expenditure & 19.8 & 28.9 & 29.6 & 19.3 & 24.8 & 26.6 & 26.4 & 30.8 & 27.4 & 17.1 & 20.8 & 27.1 & 22.5 & 8.7 & 27.9 & 8.0 & 21.0 & 21.9 & 22.0 & 21.7 & 26.7 & 19.5 & 24.7 & 26.6 & 22.4 & 17.6 & & \\
\hline Indirect taxes (on cash benefits) & 0.8 & 3.1 & 2.8 & 0.7 & 2.7 & 3.0 & 2.8 & 2.7 & 2.2 & 1.3 & 2.3 & 2.2 & 0.8 & 0.3 & 3.8 & 0.2 & 1.7 & 1.4 & 2.3 & 2.8 & 2.7 & 1.8 & 1.9 & 2.3 & 1.4 & 0.3 & 1.9 & \\
\hline 3 Net direct public social expenditure & 18.9 & 25.8 & 26.8 & 18.5 & 22.1 & 23.6 & 23.6 & 28.1 & 25.1 & 15.8 & 18.5 & 24.9 & 21.8 & 8.3 & 24.1 & 7.9 & 19.3 & 20.4 & 19.6 & 19.0 & 24.0 & 17.7 & 22.9 & 24.3 & 21.0 & 17.3 & & \\
\hline - $\quad$ Indirect taxes & 0.1 & 0.0 & 0.1 & 0.1 & 0.1 & 0.0 & 0.0 & 0.2 & 0.2 & 0.0 & 0.1 & 0.0 & 0.0 & 0.1 & 0.0 & 0.1 & 0.2 & 0.0 & 0.0 & 0.0 & 0.2 & 0.0 & 0.0 & 0.0 & 0.0 & 0.0 & & \\
\hline 4 Net TBSPs similar to cash benefits & 0.8 & 0.1 & 0.6 & 1.4 & 0.6 & 0.0 & 0.0 & 1.0 & 1.3 & 0.0 & 0.2 & 0.2 & 0.6 & 0.7 & 0.0 & 0.9 & 0.8 & 0.0 & 0.1 & 0.1 & 1.1 & 0.0 & 0.3 & 0.0 & 0.3 & 0.7 & & \\
\hline + T2 TBSPs towards current private benefits & 0.2 & 0.0 & 0.0 & 0.2 & 0.1 & 0.0 & 0.0 & 0.0 & 0.5 & 0.0 & 0.3 & 0.1 & 0.0 & 0.0 & 0.0 & 0.2 & 0.1 & 0.1 & 0.0 & 0.0 & 0.1 & 0.1 & 0.4 & 0.0 & 0.1 & 1.5 & & \\
\hline 5 Net TBSPs (not including pensions) & 1.0 & 0.1 & 0.6 & 1.6 & 0.6 & 0.0 & 0.0 & 1.1 & 1.8 & 0.0 & 0.5 & 0.3 & 0.6 & 0.7 & 0.0 & 1.1 & 0.9 & 0.1 & 0.1 & 0.1 & 1.2 & 0.1 & 0.7 & 0.0 & 0.5 & 2.2 & 0.6 & \\
\hline 6 Net current public social expenditure & 19.9 & 25.9 & 27.4 & 20.2 & 22.8 & 23.6 & 23.6 & 29.2 & 27.0 & 15.8 & 19.0 & 25.2 & 22.4 & 9.1 & 24.1 & 9.0 & 20.3 & 20.5 & 19.7 & 19.1 & 25.2 & 17.8 & 23.6 & 24.3 & 21.4 & 19.4 & 21.4 & $23 \%$ \\
\hline 7 Gross mandatory private soc. Exp. & 0.6 & 1.0 & 0.0 & 0.0 & 0.3 & 0.3 & 0.0 & 0.4 & 1.2 & 1.9 & 0.0 & 1.9 & 0.7 & 0.7 & 0.4 & 0.0 & 0.7 & 0.0 & 1.4 & 0.0 & 0.5 & 0.2 & 0.0 & 0.4 & 0.9 & 0.3 & 0.5 & \\
\hline Direct taxes and social contributions & 0.1 & 0.2 & 0.0 & 0.0 & 0.0 & 0.1 & 0.0 & 0.0 & 0.4 & 0.3 & 0.0 & 0.2 & 0.0 & 0.0 & 0.1 & 0.0 & 0.2 & 0.0 & 0.4 & 0.0 & 0.0 & 0.0 & 0.0 & 0.1 & 0.1 & 0.0 & & \\
\hline Indirect taxes & 0.0 & 0.1 & 0.0 & 0.0 & 0.1 & 0.0 & 0.0 & 0.1 & 0.1 & 0.3 & 0.0 & 0.2 & 0.0 & 0.1 & 0.1 & 0.0 & 0.1 & 0.0 & 0.2 & 0.0 & 0.1 & 0.0 & 0.0 & 0.1 & 0.1 & 0.0 & & \\
\hline 9 Net publicly mandated soc. exp. $[6+8]^{a}$ & 20.4 & 26.5 & 27.4 & 20.2 & 23.0 & 23.7 & 23.6 & 29.5 & 27.7 & 17.1 & 19.0 & 26.7 & 23.0 & 9.7 & 24.3 & 9.0 & 20.7 & 20.5 & 20.5 & 19.1 & 25.6 & 18.0 & 23.6 & 24.5 & 22.1 & 19.7 & 21.7 & $22 \%$ \\
\hline 10 Gross voluntary private soc. exp. & 4.1 & 1.2 & 5.6 & 6.2 & 0.2 & 2.7 & 1.3 & 2.9 & 2.1 & 4.4 & 2.0 & 0.7 & 3.8 & 2.4 & 0.9 & 0.2 & 7.2 & 0.6 & 0.9 & 0.0 & 1.6 & 1.1 & 0.6 & 2.8 & 5.5 & 11.4 & 2.8 & \\
\hline Direct taxes and social contributions & 0.6 & 0.1 & 0.4 & 0.8 & 0.0 & 0.9 & 0.2 & 0.0 & 0.2 & 0.6 & 0.1 & 0.0 & 0.0 & 0.0 & 0.1 & 0.0 & 1.3 & 0.0 & 0.2 & 0.0 & 0.0 & 0.0 & 0.0 & 0.6 & 0.7 & 0.7 & & \\
\hline Indirect taxes & 0.2 & 0.1 & 0.7 & 0.4 & 0.0 & 0.4 & 0.2 & 0.1 & 0.1 & 0.8 & 0.2 & 0.0 & 0.2 & 0.0 & 0.2 & 0.0 & 0.9 & 0.0 & 0.2 & 0.0 & 0.0 & 0.1 & 0.0 & 0.4 & 0.6 & 0.2 & & \\
\hline 11 Net current voluntary private soc. exp. & 3.2 & 1.0 & 4.5 & 5.0 & 0.2 & 1.4 & 0.9 & 2.8 & 1.7 & 3.0 & 1.7 & 0.6 & 3.6 & 2.4 & 0.7 & 0.2 & 5.0 & 0.6 & 0.5 & 0.0 & 1.6 & 0.9 & 0.6 & 1.7 & 4.2 & 10.6 & 2.3 & \\
\hline 12 Net current private soc. exp. $[8+11]$ & 3.7 & 1.6 & 4.5 & 5.0 & 0.4 & 1.6 & 0.9 & 3.1 & 2.4 & 4.3 & 1.7 & 2.1 & 4.2 & 2.9 & 0.9 & 0.2 & 5.5 & 0.6 & 1.3 & 0.0 & 2.0 & 1.1 & 0.6 & 2.0 & 4.9 & 10.9 & & \\
\hline 13 Net total social expenditure $[\mathbf{6}+\mathbf{1 2 - T 2}]^{\mathrm{b}}$ & 23.4 & 27.5 & 31.9 & 25.0 & 23.1 & 25.2 & 24.4 & 32.3 & 28.9 & 20.1 & 20.4 & 27.2 & 26.6 & 12.0 & 25.0 & 9.1 & 25.6 & 21.0 & 21.1 & 19.1 & 27.1 & 18.8 & 23.8 & 26.3 & 26.2 & 28.8 & 23.8 & $22 \%$ \\
\hline Memorandum item & & & & & & & & & & & & & & & & & & & & & & & & & & & & \\
\hline TBSPs towards pensions $^{\circ}$ & 3.3 & 0.1 & 0.2 & 2.3 & 0.1 & .. & 0.1 & 0.0 & 1.0 & 1.2 & 1.6 & 0.0 & 0.9 &.. & 0.8 & 0.2 & 2.1 & .. & 0.6 & .. & 0.1 & 0.2 & 0.3 & 0.0 &.. & 0.9 & .. & \\
\hline Average indirect tax rate & 0.0 & 0.0 & 0.0 & 0.0 & 0.0 & 0.0 & 0.0 & 0.0 & 0.0 & 0.0 & 0.0 & 0.0 & 0.0 & 0.0 & 0.0 & 0.0 & 0.0 & 0.0 & 0.0 & 0.0 & 0.0 & 0.0 & 0.0 & 0.0 & 0.0 & 0.0 & 0.0 & \\
\hline
\end{tabular}

a) Numbers in square brackets refer to line numbers in the second column; “.." cell with no information.

b) In order to avoid double counting, the value of TBSPs towards "current" private social benefits has been ignored for the calculation of net total social expenditure.

c) Because of conceptual issues and gaps in data availability, tax breaks towards old-age pensions are shown in the table as a memorandum item. 
A.I.2.3. Trends in Gross and Net Social Expenditure

Chart A.12.3: Gross and net social expenditure trends
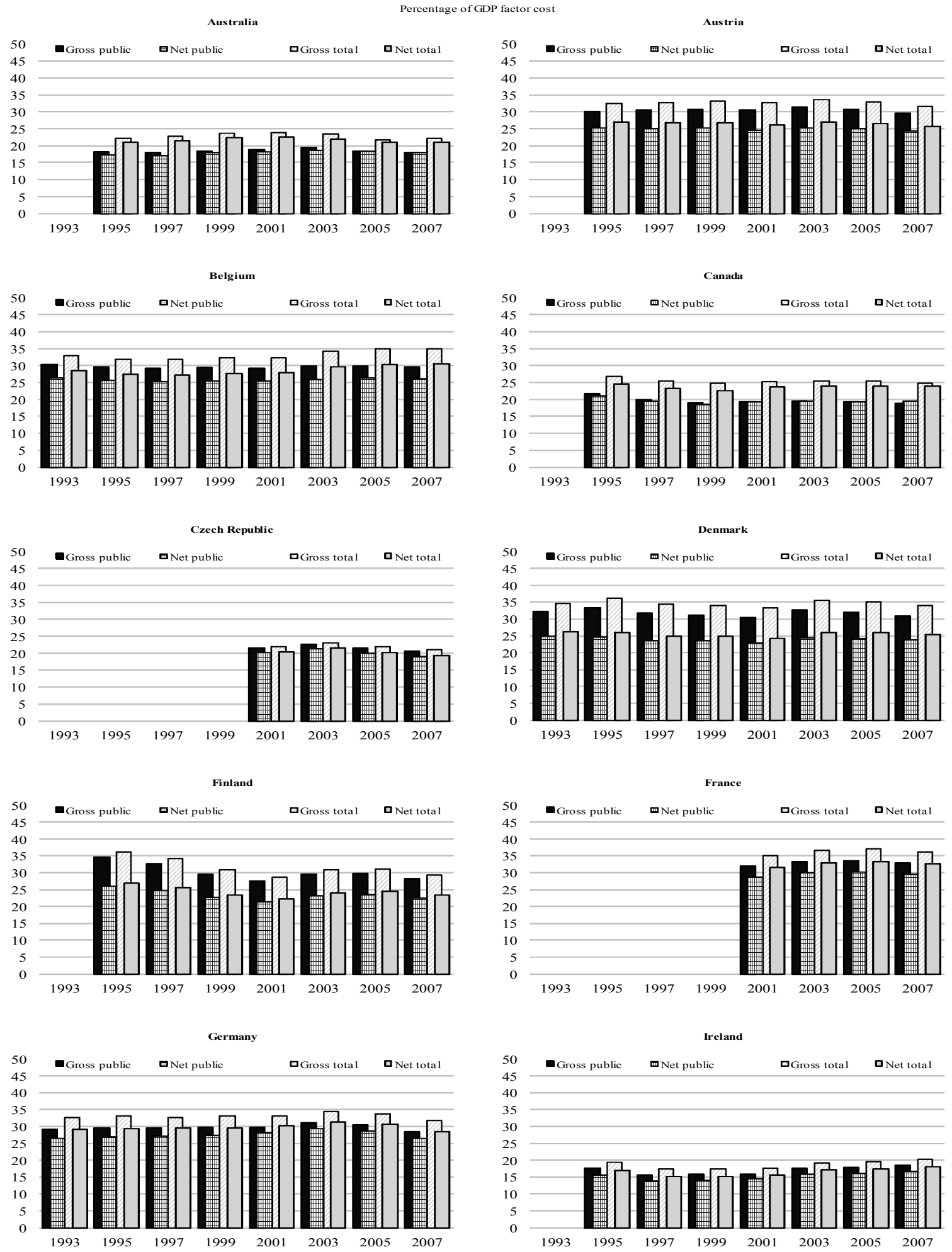
Chart A.I.2.3. Gross and net social expenditure trends (continued)
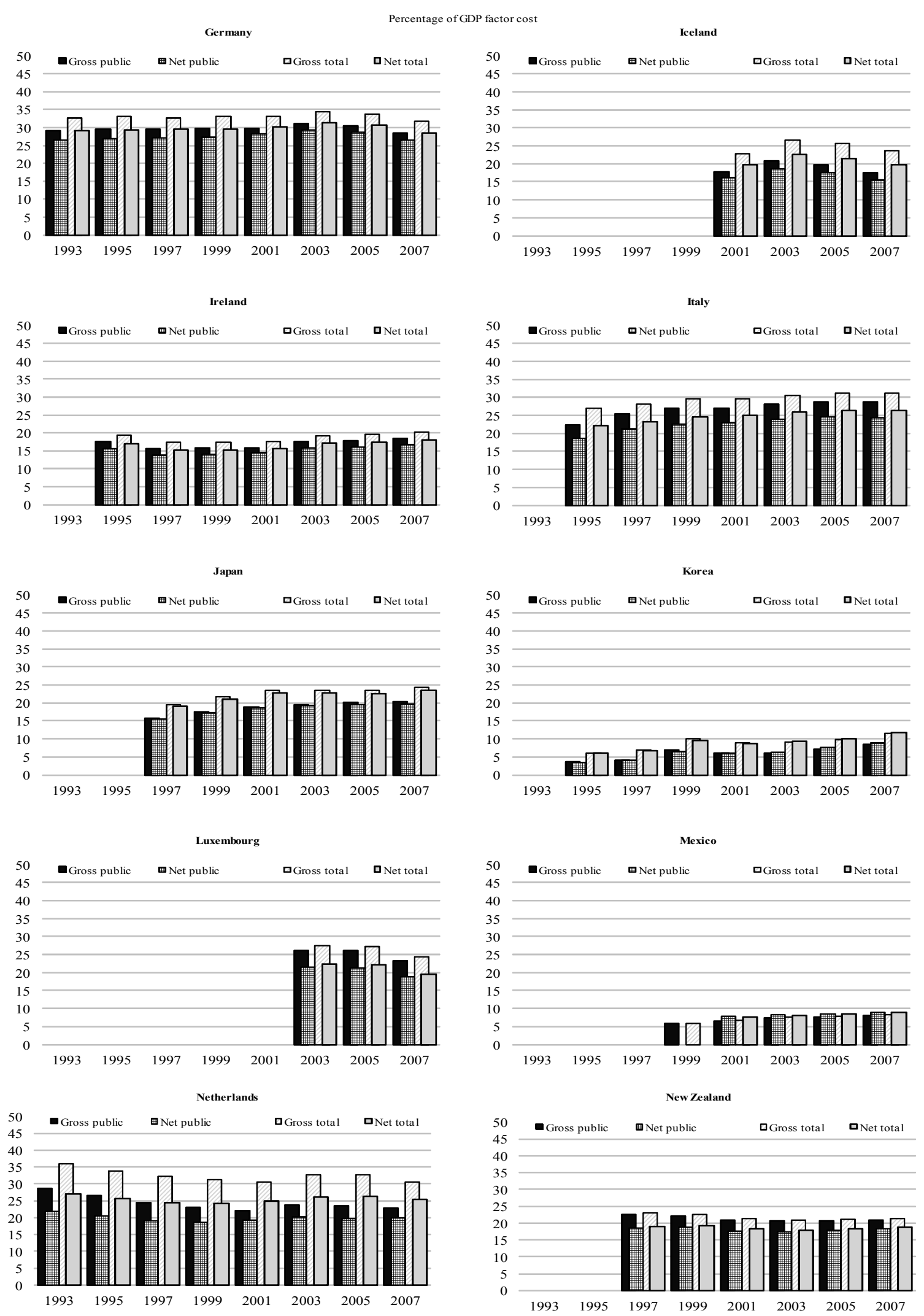
Chart A.12.3. Gross and net social expenditure trends (continued)
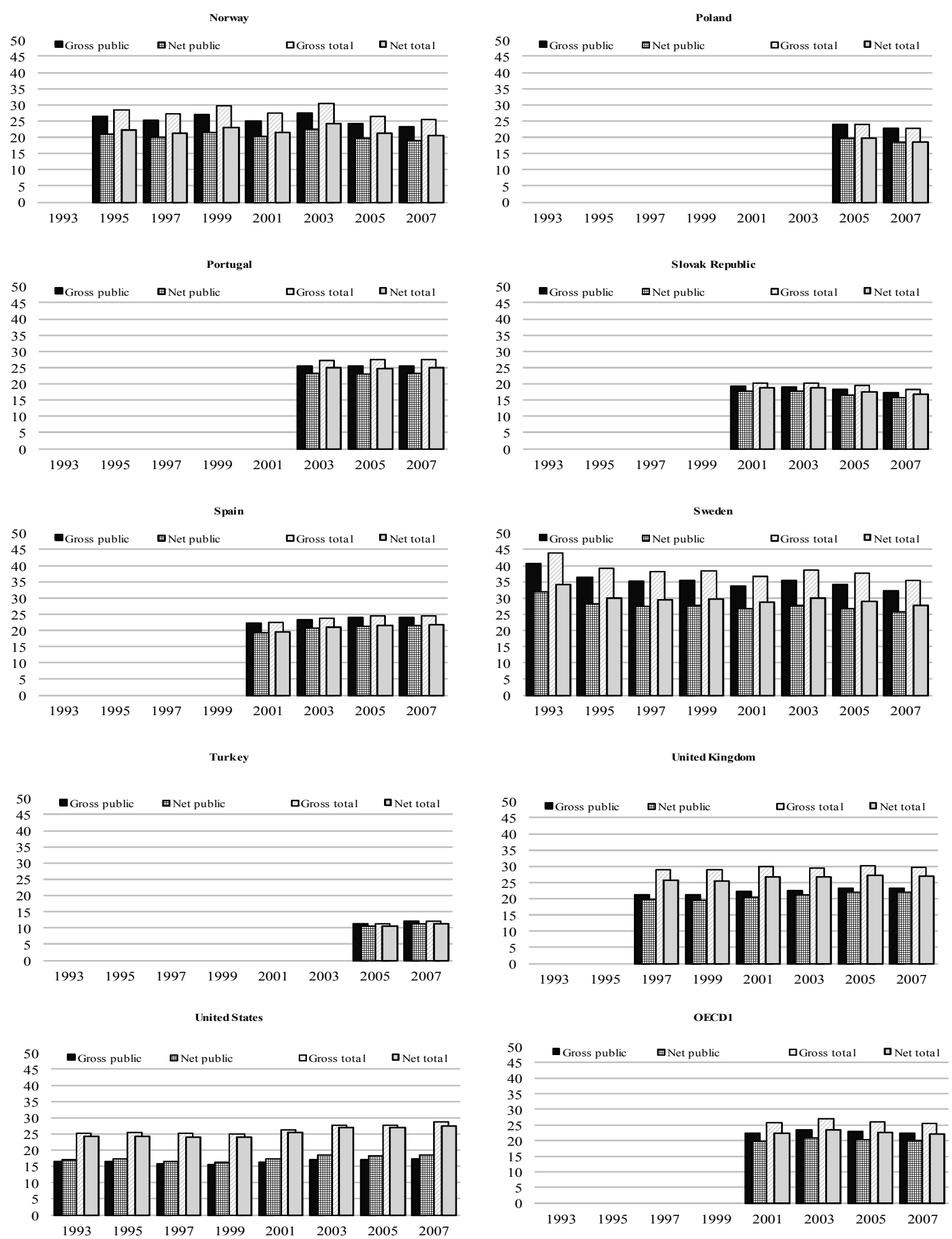

1. 2001: OECD23, 2003: OECD24, 2005 and 2007: OECD27

Source: See Table I.4. and Annex I.2. 


\section{PART II: THE SOCX MANUAL}

\section{II.1. Introduction}

63. The OECD Social Expenditure database was developed in the 1990s to facilitate social policy analysis (OECD, 1996). ${ }^{8}$ In principle the System of National Accounts (SNA) provides a comprehensive accounting framework for social expenditure and its financing (SNA, 1993). In practice, however, the aggregate nature of data included in 'social transfers' (cash and in kind) in the SNA proved inadequate for analysis of public social policy programmes and trends ${ }^{9}$ : in the context of its work-programme on public spending the Secretariat tried unsuccessfully to establish on a comprehensive basis what spending items were included in the (sub-)aggregate spending amounts recorded as government outlays by function in the National Accounts (Varley, 1986, and Oxley et al., 1990). As a result, when the OECD Social Expenditure database (SOCX) was set up in the early 1990s, it was designed to be transparent through the recording of spending items at a detailed level: the 'social expenditure programme' for all 34 OECD countries in national currency. For example, SOCX includes information for 50 separate social programmes for Canada, 65 for both the Netherlands and the US, and 300 for France. The detailed nature of expenditure data in SOCX constitutes an important form of quality control as the high level of transparency associated with detailed recording limits the scope for inappropriate recording (including double counting) of spending items in SOCX.

64. The detailed information on social expenditure items included in SOCX permits a variety of types of analysis of the effects of social policy to be undertaken. The detail in SOCX allows for in-depth study of national and cross-national social protection policy, as for example in the OECD Economic Surveys of individual member countries, and also allows for a grouping of expenditures to match the analytical needs of users, as for example: using different definitions of active social policy; an assessment of spending on all incapacity-related support programmes; an evaluation of expenditures targeted primarily at different age groups, etc. Both OECD analysts and external researchers make extensive use of information on trends and changes in the composition of social spending as in SOCX, for example: Caminada and Goudszwaard (2005); Castles (2004, 2008); Castles and Obringer (2007); Darby and Melitz (2007); Pearson and Martin (2005); Siegel (2005); Townsend (2007); Whiteford and Adema (2007); Kirkegaard (2009); Fishback (2010); Adema and Whiteford (2010); and, OECD (2011a) . Prior to the 2010 release of SOCX the OECD produced six updated volumes of the database since the initial release; OECD (1999; 2000a; and 2001) via CD-ROM, while OECD (2004, 2007a and 2009) were released through the OECD Internet.

For the regular data collection for the National Accounts, countries only report two items that are directly related to public social expenditure: 1) social transfers in cash (D62); and 2) social transfers in kind (D63). Data recorded for the Classification of Function of Government (COFOG) typically record four public social expenditure items (spending by general government, central government, local government and social security funds, see OECD, 2010a), although national sources may provide more detail. For example, Statistics Canada reports about 20 items on public social transfers in Canada (www.statcan.ca). 
65. SOCX also presents the aggregated public and private social expenditure grouped along nine social policy areas, and to facilitate international comparisons this information is related to gross domestic product, gross national income, total government expenditure, and in purchasing power parities per head. SOCX does not contain information on the financing of social programmes on a comprehensive basis.

66. The OECD has developed different and more comprehensive measures of the resources devoted to social policies in OECD countries; indicators on net (after tax) total (public and private) social expenditure. This work started in the mid-1990s with initial estimates on net public social expenditure for six countries (Adema et al., 1996), but over the years the methodological framework and available data have been extended to cover 27 OECD countries: Australia, Austria, Belgium, Canada, the Czech Republic, Denmark, Finland, France, Germany, Iceland, Ireland, Italy, Japan, Korea, Luxembourg, Mexico, the Netherlands, New Zealand, Norway, Poland, Portugal, the Slovak Republic, Sweden, Spain, Turkey, the United Kingdom and the United States (detailed information per country is presented in Annex I.2). This work is undertaken in close collaboration with the OECD Centre for Tax Policy and Administration, and these indicators are treated as an integral part of SOCX, and will be updated as the rest of the database, i.e., every two years. The next collection round is scheduled to start in 2011, with results to be released in 2012 .

67. The OECD Social Expenditure database (SOCX) has been designed to be compatible with the System of National Accounts and inter alia the System of Health Accounts (OECD, $2000 b$ and SNA, 1993). It is also broadly compatible with Eurostat's European System of Social Protection Statistics ESSPROS, and the ILO Social Security Inquiry - SSI (Box II.1; Eurostat, 2008, and ILO, 2005). Information on social expenditure and recipiency of social support that is collected by the Asian Development Bank as part of its Social Protection Index initiative is also broadly compatible with the other databases (ADB, 2006 and 2008).

\section{Box II.1: The relationship between OECD, Eurostat and ILO social accounting systems}

Compared to SOCX, the scope of Eurostat's European System of Social Protection Statistics - ESSPROS (via http://epp.eurostat.ec.europa.eu/portal/page/portal/social_protection/data) and the ILO's Social Security Inquiry - SSI (via www.ilo.org/dyn/ilossi/ssimain.home), is wider as these systems also include information on financing of social expenditure. From a statistical perspective it may be desirable that the OECD Social Expenditure database is extended to include information on the financing of social programmes that is consistent with the OECD Revenue Statistics (OECD, 2010a), but the resources that would be required for such an exercise are likely to far exceed the gains that could be made in terms of strengthening policy analysis.

In terms of social domain, the OECD has arguably the largest scope as it has developed a methodology, which facilitates the comprehensive accounting of fiscal measures that affect social protection (see below). In terms of gross spending items, the SSI has a relatively large scope as it includes spending supporting basic education, as for example spending on school-books (SOCX reports public spending on education as a memorandum item, see Annex I.4). The scope of ESSPROS is narrower than that of SOCX and the SSI as it focuses on support that can be 'allocated' to individuals and, consequently, it does not include all spending on public health expenditures or labour market programmes. The ILO and the OECD both record spending on Active Labour Market Policies, with the OECDdefinitions being the least restrictive as they include government subsidies towards the cost of employment of previously unemployed persons.

Functional categorisations in ESSPROS (Eurostat, 2008) and the Social Security Inquiry (ILO, 2005) are also slightly different from each other. ESSPROS groups items in 7 functions; the SSI identifies 11 functions; and SOCX has 9 social policy areas at present. 


\section{II.2. Defining the social domain}

68. To facilitate cross-country comparisons of social expenditure, the first step is to demarcate what spending is 'social' and what is not. The OECD defines social expenditures as:

"The provision by public and private institutions of benefits to, and financial contributions targeted at, households and individuals in order to provide support during circumstances which adversely affect their welfare, provided that the provision of the benefits and financial contributions constitutes neither a direct payment for a particular good or service nor an individual contract or transfer."

69. Since only benefits provided by institutions are included in the social expenditure definition, transfers between households - albeit of a social nature, are not in the social domain. ${ }^{10}$

70. Social benefits include cash benefits (e.g., pensions, income support during maternity leave and social assistance payments), social services (e.g., childcare, care for the elderly and disabled) and tax breaks with a social purpose (e.g., tax expenditures towards families with children, or favourable tax treatment of contributions to private health plans).

71. There are two main criteria which have to be simultaneously satisfied for an expenditure item to be classified as social. First, the benefits have to be intended to address one or more social purposes. Second, programmes regulating the provision of benefits have to involve either a) inter-personal redistribution, or b) compulsory participation.

\section{II.2.1. Towards a social purpose}

72. The OECD Social Expenditure Database groups benefits with a social purpose in nine policy areas (see also section II.3.1 for more detail):

- Old-age - pensions (see Box II.2), early retirement pensions, home-help and residential services for the elderly;

- Survivors - pensions and funeral payments;

- Incapacity-related benefits - care services, disability benefits, benefits accruing from occupational injury and accident legislation, employee sickness payments;

- Health - spending on in- and out-patient care, medical goods, prevention;

- Family - child allowances and credits, childcare support, income support during leave, sole parent payments;

- Active labour market policies - employment services, training, employment incentives, integration of the disabled, direct job creation, and start-up incentives;

- Unemployment - unemployment compensation, early retirement for labour market reasons;

- Housing - housing allowances and rent subsidies; and,

- Other social policy areas - non-categorical cash benefits to low-income households, other social services; i.e., support programmes such as food subsidies, which are prevalent in some non-OECD countries.

10 Social spending does not include remuneration for work, as it does not cover market transactions, i.e., payments in return for the simultaneous provision of services of equivalent value. Employer costs such as allowances towards transport, holiday pay, etc. are part of remuneration in this sense. 


\section{Box II.2: Earnings and deferred wages; the treatment of pensions and severance payments in SOCX}

The definition of social spending explicitly rules out remuneration for work, and therefore items as holiday pay, costs incurred for transport to work and bonuses are not covered in the database. The exclusion of remuneration for current work effort from the social spending remit is uncontested, but what about "remuneration for past work or deferred wages"? In fact, a substantial part (i.e., that part financed by employer contributions) of the pension payments by public and private pension funds can be argued to concern deferred wages. If social expenditure were not to include any such items then almost all pension payments would be excluded from SOCX, and other relevant databases as operated by, for example, Eurostat and the ILO. Instead, by convention, pensions (in general payments of people above retirement age), are considered to be part of social expenditure, also when co-financed by past employer contributions.

If pensions are considered to be social expenditure then the question arises which other similar payments should also be included in the database. General saving plans are often used for retirement, but it is unclear to what extent this is the case. Similarly, life insurance saving instruments across the OECD are also used for the same reason, but, again, there is insufficient detail in the available data to establish which particular programme or savings vehicle is geared towards retirement. Hence, such data are not included in SOCX.

Severance payments can also be used for retirement, and if pensions are included in SOCX, it would be consistent to also include severance payments if they are made towards retirement. However, severance payments are not exclusively made for retirement purposes. Severance payments are made when an employment relationship between employer and employee ceases to exist, and that can also be because an employee quits voluntarily or is dismissed.

In its balance of methodological choices, SOCX treats severance payments on retirement as retirement allowances similar to pensions, while severance payments to people below the normal retirement age are considered as separation payments and treated as remuneration. There is one exception: the OECD Labour Market Policy database and SOCX include "redundancy compensation", when such payments are made by public funds to workers "who have been dismissed through no fault of their own by an enterprise that is ceasing or cutting down its activities". This covers a small and specific group of all "severance payments", which are included under unemployment compensation.

In theory, SOCX should include that part of spending of the severance pay which is given to people who reach retirement, and exclude the rest. However, such a level of detail is generally not available, and choices on whether or not to include severance payments had to be made on a case-by-case basis.

By and large this issue is most relevant to the following three countries:

i) Spending on severance payments is worth about $1 \%$ of GDP in total in Italy and can be split in payments to (former) public and private employees. For public sector workers, available data confirm that the vast majority of payment is paid on retirement of the employee (INPDAP, 2008). While there are no statistics on the age of the severance payments to private sector employees, ".... a significant majority of the aggregate amount of benefit is paid out to people who are retiring...". Eurostat therefore continues to classify the Italian severance payments in its Old Age function.

ii) New evidence from Japan suggests that voluntary private severance payments amounted to $2.9 \%$ of GDP in 2007 (relevant statistics on severance payments/retirement income are published in the Tax Statistics published by the National Tax Office in Japan). The Japanese authorities assume that the majority of recipients of severance pay receive these payments on retirement, even though the statistics do not allow for an exact identification of that percentage.

iii) In Korea, total severance payments amount to $2.0 \%$ of GDP. The majority of severance payments are being made when workers are laid off or quit voluntarily before compulsory retirement age. Korean policy aims to convert severance payments into a corporate pension saving, leading to the so-called "Retirement Pension Benefits". However, while the government provides tax incentives to stimulate conversion, it is not mandatory, and the proportion of enterprises involved is around 15\% (Ministry of Labor, 2009). Only a minority of all employer-paid severance payments (around 10 to $30 \%$ at maximum) concerned workers who retired, and therefore SOCX includes $20 \%$ (equivalent to $0.4 \%$ of GDP) of all spending on severance payments under mandatory private old-age expenditure. When in future more detailed information on severance payments made on retirement and the amount of Retirement Pension Benefits becomes available, such spending will be included in SOCX.

In sum, as most spending on severance payments in Italy and Japan seems to be made on retirement they are included in SOCX. For Korea, it is the other way round as only a minority of spending on severance pay goes to people reaching retirement. The error of including all severance payments under old age spending would be larger than when no such spending was recorded for Korea at all. To further reduce the margin of error SOCX includes $20 \%$ of all spending on severance payments until more comprehensive information on the issue becomes available. 
73. The borderline of the social domain is not always immediately clear because policy objectives differ across countries. Tackling child poverty is an important policy objective in all OECD countries, and support for children (either through cash transfers, services or through the tax system) is considered as social. However, favourable fiscal treatment of marital status is not considered as social support in the OECD Social Expenditure database, as there is no OECD-wide agreement on whether such support reflects the pursuit of social policy objectives (across countries there are also different views on the basic economic unit, which is the appropriate basis for taxation).

74. In practice, data issues also play a role in determining whether certain items are considered social or not. For example, when saving programmes are earmarked towards income support in retirement (or towards contingencies covered by other social policy areas), they are considered to be 'social'.

75. Rent subsidies are considered social, as is residential support for the elderly, disabled and other population groups (as recorded under Old-age, Incapacity-related benefits, etc.). Mortgage relief for lowincome households has some similarities with such programmes. However, it is unclear up to what level of income, or what level of property value, such support should be considered social. Relevant thresholds differ across countries, while, in any case, comprehensive cross-national data are not available. For these reasons, mortgage relief and capital subsidies towards construction of housing are not considered here.

76. For this issue of SOCX public expenditure on childcare and early educational services has been taken from national statistics, Eurostat and the annual (OECD/Eurostat) data collection on (pre-primary) education (OECD, 2010b). In order to get a better comparison of childcare support, indicators have been adjusted for cross-national differences in the compulsory age of entry into primary school. For example, in some (Nordic) countries children enter primary school at age 7, while attending pre-primary schooling the year beforehand. In order to improve the comparison, expenditure on these 6-year-olds was excluded (sometimes using estimates derived on basis of available data on spending on education and the number of 6-year-olds). Similarly, for countries where children enter school at age 5 (and which are not included in the childcare and pre-school data) pre-school expenditure data for Australia, New Zealand and the United Kingdom was adjusted by adding up the expenditure corresponding to 5 -year-old children enrolled in primary school.

77. Nevertheless, there remain weaknesses in spending data, not least because local governments often play a key role in financing childcare services. This does not lead to recording issues in Nordic countries, but in other (often federal) countries, it is much more difficult to get a good view of public support for childcare across a country. This is because local governments may use different funding streams to finance childcare services, e.g., non-earmarked general block-grants, as in Canada, or because information on spending by local governments on childcare is not reported to national authorities, e.g., Switzerland. These issues are not restricted to federal countries. In the Netherlands, municipalities can provide childcare support for (groups) of their inhabitants, and they may finance this out of the general block-grant to municipalities. They can also use the central government funding stream to municipalities to support labour market integration for income support recipients, to finance, for example, childcare support for social assistance clients.

\section{II.2.2. Inter-personal redistribution or compulsion}

78. Expenditure programmes are considered 'social' if participation is compulsory, and if entitlements involve inter-personal redistribution of resources among programme participants; in other words, if entitlements are not the result of direct market transactions by individuals given their individual risk profiles. The provision of social services (by public authorities and/or non-government organisations) and social insurance and social assistance programmes practically always involves redistribution across households. Such programmes are either financed through general taxation or social security contributions, 
which lead to the redistribution of resources across the population or within population groups (e.g., all members of an unemployment insurance fund).

79. Inter-personal redistribution in private programmes is often introduced by government regulation or fiscal intervention. Governments may force individuals and/or employers to take up protection provisions regardless of their risk-profiles or the prevailing market prices. For example, through risksharing (e.g., through forcing insurance companies to have one price for both sick and healthy people) public policy can subsidise sick people, and thus ensure redistribution between households. Public fiscal intervention to stimulate private take-up on a collective or individual basis also means that the take-up decision is not fully determined by the individual risk-profile or prevalent market prices (the same holds for social benefits derived from collective agreements or taken out by employers on a collective basis). There is a high degree of similarity between legally-stipulated private schemes and tax-advantaged plans.

80. Social benefits are also defined to include some (public and private) pension programmes that in theory do not necessarily involve redistribution of resources across households as, for example, the compulsory government managed individual savings scheme in Singapore (Ramesh, 2005). This is because just as with the provision of tax relief, compulsion reflects a policy judgement that coverage of these plans is desirable; hence, these programmes are considered social.

\section{II.2.3. Public, private social and exclusively private expenditure}

81. The distinction between public and private social protection is made on the basis of whoever controls the relevant financial flows; public institutions or private bodies. Public social expenditure: social spending with financial flows controlled by General Government (different levels of government and social security funds), as social insurance and social assistance payments. For example, sickness benefits financed by compulsory employer and employee contributions (receipts) to social insurance funds are by convention considered public. In line with SNA93 ${ }^{11}$, SOCX records pensions paid to former civil servants through autonomous funds as a private spending item (Australia (partially ${ }^{12}$ ), Canada, Denmark, the Netherlands, Sweden and the United Kingdom). All social benefits not provided by general government are considered 'private'.

82. Within the group of private social benefits, additional two broader categories can be distinguished:

- $\quad$ Mandatory private social expenditure: social support stipulated by legislation but operated through the private sector, e.g., direct sickness payments by employers to their absent employees as legislated by public authorities, or benefits accruing from mandatory contributions to private insurance funds.

SNA (1993), para 8.63 states: “... Social insurance schemes organized by government units for their own employees, as opposed to the working population at large, are classified as private funded schemes or unfunded schemes as appropriate and are not classified as social security schemes. ..." In practical terms, for pension payments to former civil servants to be classified as private, these payments have to go through autonomous private funds (e.g., separate pension and/or insurance companies), for which the government does not make up the deficit on a regular basis (e.g., in practice benefit schemes which are defined contributions plans). Non-autonomous pension schemes (including pension benefits paid directly from the government budget) remain institutionally in the government sector.

12 The Australian pension arrangements for former civil servants constitute a hybrid of public and private components. The relevant pension payment is a defined benefit scheme which is guaranteed by the government and thus classified as public. In contrast, the lump-sum payment which many civil servants take on retirement is based on their compulsory contributions and interest rates; relevant spending has been grouped under mandatory private social expenditure for Australia. 
- Voluntary private social expenditure: benefits accruing from privately operated programmes that involve the redistribution of resources across households and include benefits provided by NGOs, and benefit accruing from tax advantaged individual plans and collective (often employment-related) support arrangements, such as for example, pensions, childcare support, and, in the United States, employmentrelated health plans. ${ }^{13}$

83. SOCX includes data on the size of private social spending across the OECD, but this data is nevertheless deemed of lesser quality than information on budgetary allocations for social support.

84. Take-up of individual insurance, even with a social purpose, is a matter for those concerned, and premiums are based on the individual preferences and the individual risk profile. For example, if someone takes out private pension insurance which is actuarially fair, then there is no ex ante redistribution across households. The insurance company sets the price so that the individual can expect to receive compensation payments in return for exactly what it costs him or her. Such spending is not considered social, but 'exclusively private'. Table II.1 summarizes which expenditures are social and which are not. Box II.3 provides further detail on issues with the categorisation of benefits with a social purpose.

Table II.1: Categorisation of benefits with a social purpose ${ }^{1,2}$

\begin{tabular}{|c|c|c|c|c|}
\hline & \multicolumn{2}{|c|}{ Public } & \multicolumn{2}{|c|}{ Private } \\
\hline & Mandatory & Voluntary & Mandatory & Voluntary \\
\hline Redistribution & $\begin{array}{c}\text { Means-tested } \\
\text { benefits, social } \\
\text { insurance benefits }\end{array}$ & $\begin{array}{l}\text { Voluntary participation } \\
\text { in public insurance } \\
\text { programmes. Self- } \\
\text { employed 'opting in' to } \\
\text { obtain insurance } \\
\text { coverage. }\end{array}$ & $\begin{array}{l}\text { Employer-provided } \\
\text { sickness benefits, benefits } \\
\text { accruing from mandatory } \\
\text { contributions, to, for } \\
\text { example, pension or } \\
\text { disability insurance. }\end{array}$ & $\begin{array}{l}\text { Tax-advantaged benefits, } \\
\text { e.g., individual retirement } \\
\text { accounts, occupational } \\
\text { pensions, employer- } \\
\text { provided health plans }\end{array}$ \\
\hline No redistribution & $\begin{array}{c}\text { Benefits from } \\
\text { government } \\
\text { managed } \\
\text { individual saving } \\
\text { schemes }\end{array}$ & & $\begin{array}{l}\text { Non tax-advantaged } \\
\text { actuarially fair pension } \\
\text { benefits }\end{array}$ & $\begin{array}{l}\text { Exclusively private: } \\
\text { Benefits accruing from } \\
\text { insurance plans bought at } \\
\text { market prices given } \\
\text { individual preferences. }\end{array}$ \\
\hline
\end{tabular}

(1) By definition transfers between individuals, even when of a social nature, are not considered to be within the social domain.

(2) The shaded cells reflect benefits that are NOT classified as social.

85. Life insurance savings plans are considered outside the social domain as comprehensive information on that part of life insurance payments which is earmarked for social purposes is not available; in fact, there is no comprehensive information on life insurance benefits. Although the practice of reinsurance makes it difficult to get a precise view on the importance of life-insurance arrangements, available information on life insurance premiums suggests that life insurance arrangements play an important role $(\mathrm{OECD}, 2010 \mathrm{c})$. To a considerable extent, life insurance policies are taken up to cover mortgage arrangements, which are not considered to serve a social purpose, but private life-insurance benefits with a social element, such as payments towards death, disability, medical interventions and retirement, can be important and are included where these are separately identifiable (see below).

13 It might be argued that only the value of the fiscal intervention towards the private pension benefit should be considered social. However, relevant fiscal measures redistribute resources up to the level where taxadvantages no longer apply, and thus all benefits accruing from such contributions should be included. 


\section{Box II.3: Identifying and categorising benefits with a social purpose}

The OECD Social Expenditure database groups social benefits by the nature of provision into public, mandatory private and voluntary private social expenditure across nine different social policy areas. All other (insurance) arrangements with a social purpose, which are based on individual risk-profiles and obtained at prevailing market prices, are outside the social domain. Examples of such arrangements that do not involve redistribution or compulsory participation are individual pension plans and individual health insurance packages.

In theory, information on the purpose of social expenditure programmes, their redistributive nature, their legal basis and control of financial flows, provides clear benchmarks for identifying public, mandatory private, voluntary private and exclusively private programmes. Sometimes classification is straightforward. For example, income support during parental leave paid by a public insurance fund is 'public'; legally required continued wage payments by employers to fathers on paternity leave are 'mandatory private', while parental leave payments made by employers on their own initiative (or because they signed up to a collective labour agreement) are voluntary private. It is more difficult when payments involve a mixture of these forms, and in the absence of good data classification, decisions have to be made.

In particular, regarding private pension funds it can be very difficult to make an unambiguous categorisation between mandatory private benefits, voluntary private benefits, and benefits that are not considered part of the social domain. Classification problems are exacerbated by the fact that contributions that underlie pension payments are made over various years and the nature of the contributions can shift over time.

Consider the case where benefit payment in year $t, B(t)$, is related to contributions in previous years, $C(t-n)$, and the rate of return on investment income, I(t-n):

$$
B(t)=F[\Sigma(C(t-n), I(t-n))]
$$

The total amount of contributions $(\mathrm{C})$ paid to a particular arrangement over the years can be the sum of different types of contributions: mandatory contributions (Cm); (Cv); and, exclusively private contributions $(\mathrm{Ce})$. In any particular year:

$$
\mathrm{C}=\mathrm{Cm}+\mathrm{Cv}+\mathrm{Ce}
$$

Thus, benefit payments in a given year can be related to four types of contributions made over previous years and the relative importance of the different types of contributions can shift from year to year.

Often, data on benefit payments only record aggregate payments $(\mathrm{Bx})$ and do not separately identify payments due to different types of contributions $(\mathrm{Cm}, \mathrm{Cv}, \mathrm{Ce})$. For example, data on pensions paid by Superannuation plans in Australia or private pension plans in Switzerland do not separately identify payments derived from mandatory private, voluntary private or exclusively private pension contributions. All Superannuation pension payments (not the lump-sum payments) to former private sector workers are grouped under voluntary private social benefits, as the pension payments that derive from mandatory contributions are currently relatively small. However, with recently increased mandatory contributions rates, pension payments deriving from mandatory contributions in Australia are expected to increase with the maturing of Superannuation plans.

Individual pension plans, for example, individual retirement accounts in the United States, are only in the (voluntary private) social domain in as much the underlying contributions were tax advantaged (in New Zealand, where favourable tax treatment concerns payments and not contributions, only the pension payments subject to taxadvantages would be included). Ideally, we would not include those private benefits that derive from non-fiscally advantaged contributions, but data, which allow for such a distinction is not always available. The decision on whether or not to include individual pension programmes is made on a case-by-case basis. For example, available tax data for the United States facilitates the identification of pensions and individual retirement disbursements, which are part of the social domain as defined above, and are therefore included in the private pension expenditure data in SOCX. 
86. There are significant differences across countries in the extent to which social policy goals are pursued through the tax system or in the role of private provision within national social protection systems (as seen above). These differences point to substantial variance in the re-distributional nature of social systems. Some private social programmes may generate a more limited redistribution of resources than public ones, and tax advantages towards private pension and health plans are more likely than not to benefit the relatively well-to-do. Private employment-related social benefits mostly re-allocate income between the (formerly) employed population, and the same holds largely true for fiscally-advantaged individuals or group retirement plans. Cross-national differences in redistribution are not just related to individual programme design, but also to the overall level of social spending. Income redistribution in a high public spending country such as Denmark tends to be larger than in, for example, the United States, where private social spending plays a much more substantial role (OECD, 2008, and Whiteford and Adema, 2007).

\section{II.3. Social expenditure programme data in SOCX}

\section{II.3.1 Categorisation of programmes across policy areas}

87. The OECD Social Expenditure Database groups benefits with a social purpose in nine policy areas - Old-age, Survivors, Incapacity-related benefits, Health, Family, Active labour market policies, Unemployment, Housing, and Other social policy areas. Table II.2 Panel A shows the structure of SOCX database for public and mandatory private programmes. Table II.2 Panel B shows the simplified structure of SOCX database for voluntary private expenditure as the quality of information is not as high as on budgetary allocations, and spending detail by programme is not available on a comprehensive basis.

Table II.2: Structure of SOCX database for public and mandatory private programmes

Panel A: Programmes by social policy area (1-9) and type of expenditure (cash / in kind)
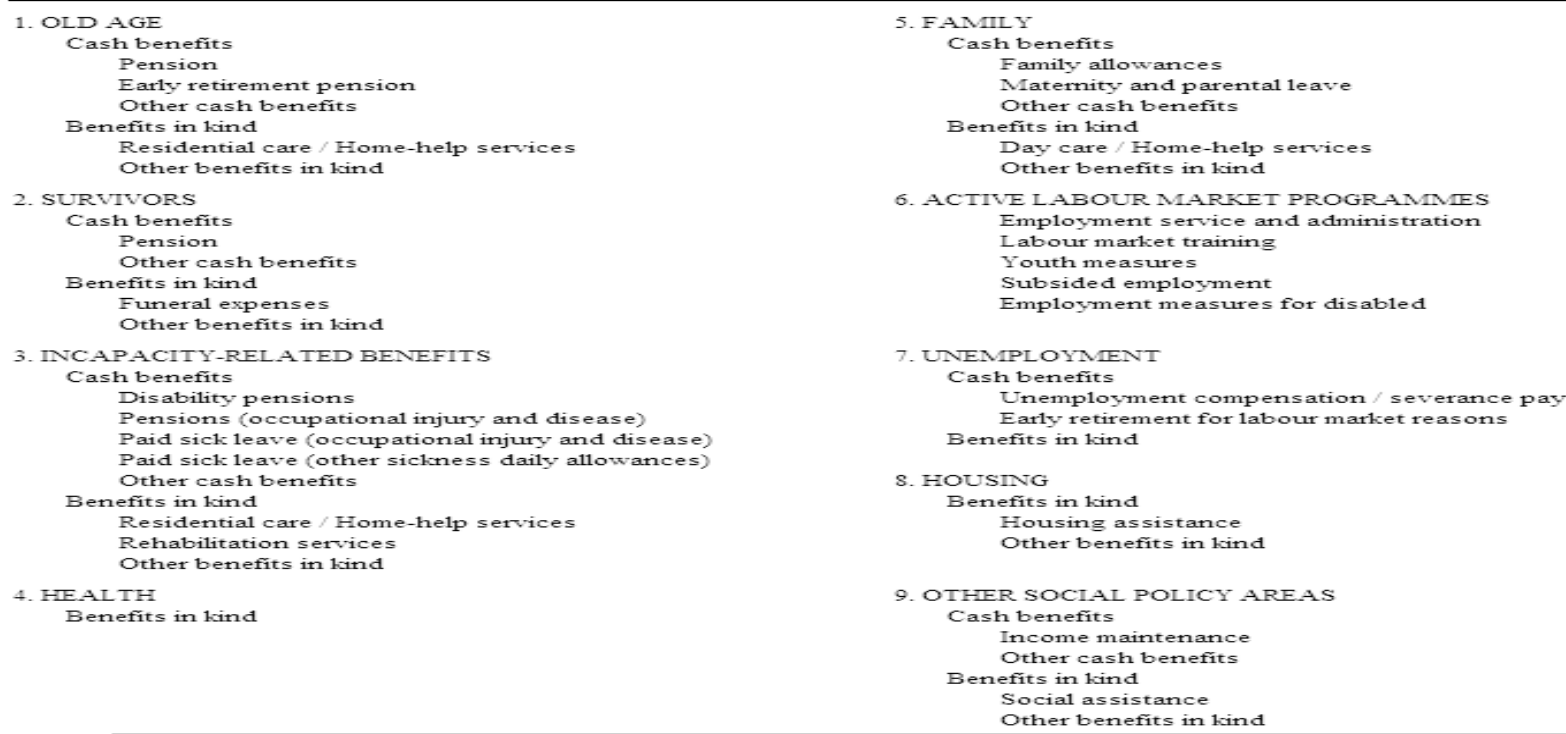

Panel B: Categorisation of voluntary private expenditure

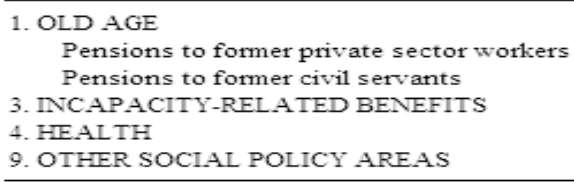


88. The nine policy areas are defined as follows; including examples of programmes (see Annex II.2 for details on codes of programmes):

- 1. Old-age - comprises all cash expenditures (including lump-sum payments) on old-age pensions. Old-age cash benefits provide an income for people retired from the labour market or guarantee incomes when a person has reached a 'standard' pensionable age or fulfilled the necessary contributory requirements. This category also includes early retirement pensions: pensions paid before the beneficiary has reached the 'standard' pensionable age relevant to the programme. Excluded are programmes concerning early retirement for labour market reasons which are classified under unemployment. Old-age includes supplements for dependants paid to old-age pensioners with dependants under old-age cash benefits. Old-age also includes social expenditure on services for the elderly people, services such as day care and rehabilitation services, home-help services and other benefits in kind. It also includes expenditure on the provision of residential care in an institution (for example, the cost of operating homes for the elderly). In order to remain consistent with the SNA93, SOCX now records pensions paid to former civil servants through autonomous funds as private spending items. Examples of programmes include:

- "250.10.1.1.1.1 Basic scheme: CNAV" is the French public basic pension scheme from "Régime général"

- "208.10.1.2.1.2 Assistance in carrying daily tasks for the elderly" is the Danish programme from municipalities that offers services to the elderly

- “392.20.1.1.1.1 Employees' pension funds" is the Japanese mandatory private occupational pension scheme

- "826.30.1.1.1.4 Public sector occupational pension" is the programme recording pension benefits to former civil servants in the United Kingdom.

- 2. Survivors - many countries have social expenditure programmes in the public sphere which provide the spouse or dependent of a deceased person with a benefit (either in cash or in kind). Expenditure in this policy area has been grouped under survivors. Allowances and supplements for dependent children of the recipient of a survivors' benefit are also recorded here. Examples of programmes include:

- “124.10.2.1.1.2 CPP and QPP: surviving spouse's pension" is the Canadian Pension Plan and Quebec Pension Plan programmes paying benefits to surviving spouses

- "348.10.2.2.1.1 Funeral expenses (means-tested)" is the Hungarian means-tested programme giving public support for funerals.

- 3. Incapacity-related benefits - disability cash benefits comprised of cash payments on account of complete or partial inability to participate gainfully in the labour market due to disability. The disability may be congenital, or the result of an accident or illness during the victim's lifetime. Spending on Occupational injury and disease records all cash payments such as paid sick leave, special allowances and disability related payments such as pensions, if they are related to prescribe occupational injuries and diseases. Sickness cash benefits related to loss of earning because of the temporary inability to work due to illness are also recorded. This excludes paid leave related to sickness or injury of a dependent child which is recorded under family cash benefits. All expenditure regarding the public provision of health care is recorded under health. Social expenditure on services for the disabled people encompasses services such as day care and rehabilitation services, home-help services and other benefits in kind. Examples of programmes include: 
- "756.10.3.1.1.1 Disability pension: invalidity insurance (non means-tested)" is the Swiss public non-means tested disability insurance pension

- "442.10.3.1.4.4 Paid sick leave" is the public programme in Luxembourg reimbursing $100 \%$ of wage (up to a ceiling) for sick blue collar employees from the first day of sickness up to three months and sick white collar employees from the third month up to the $12^{\text {th }}$ month of sickness

- "578.20.3.1.4.1 Sickness and waiting period benefit" is an estimation of mandatory benefits paid by employers in Norway during the first two weeks of sickness

- "752.30.3.0.0.0 Incapacity-related benefits" include Swedish voluntary private contractual disability pensions.

- 4. Health - social expenditure data in the health policy area is taken from the OECD Health Data (OECD, 2010d). All public expenditure on health is included (not total health expenditure): current expenditure on health (personal and collective services and investment). Expenditure in this category encompasses, among other things, expenditure on in-patient care, ambulatory medical services and pharmaceutical goods. Individual health expenditure, insofar as it is not reimbursed by a public institution, is not included. As already noted, cash benefits related to sickness are recorded under sickness benefits. Voluntary private social health expenditure are estimates on the benefits to recipients that derive from private health plans which contain an element of redistribution (such private health insurance plans are often employment-based and/or tax-advantaged).

In this and the previous versions of SOCX, efforts have been made to limited double counting of spending on long-term care as reported by health and social policy authorities. In particular, recent improvements in the System of Health Accounts have brought greater transparency and better recording in this area (OECD, 2010d, 2000b and www.oecd.org/health/sha). For more information see Annex II.1.2.

- 5. Family - includes expenditure which supports families (i.e., excluding one-person households). This expenditure is often related to the costs associated with raising children or with the support of other dependants. Expenditure related to maternity and parental leave is grouped under the family cash benefits sub-category (OECD Family database - Indicator PF1.1 and PF3.1 - www.oecd.org/els/social/family/database). Examples of programmes include:

- “56.10.5.1.1.1 Family allowance: National office for employees' family allowances" is the Belgian public programme giving child benefits to families

- "246.10.5.1.2.2 Maternity and parent's allowance" is the social security programme of income maintenance in the event of childbirth in Finland

- “203.10.5.2.1.6 Child care (pre-primary education)" is public spending in the Czech Republic towards formal day-care and pre-school services for children not yet 6 years of age. To get a good comparison of support for early care and education services (be it in (family) day-care, pre-school or, in some countries, school settings) account has been taken of cross-national differences in the compulsory age of entry into primary school. For example, in some (Nordic) countries children enter primary school at age 7, while 6-year-olds attend preprimary school the year before. In order to improve the comparison, expenditure on these 6year-olds was excluded (sometimes using estimates derived on basis of available data on spending on education and the number of 6-year-olds). Similarly, for countries where children enter school at age 5 (and which were not already included in the childcare and pre- 
school data) pre-school expenditure data for Australia, New Zealand and the United Kingdom was adjusted by adding up the expenditure on 5-year-olds enrolled in primary school (see also, the OECD Family database - Indicator PF3.1 www.oecd.org/els/social/family/database).

- 6. Active labour market programmes - contains all social expenditure (other than education) which is aimed at the improvement of the beneficiaries' prospect of finding gainful employment or to otherwise increase their earnings capacity. This category includes spending on public employment services and administration, labour market training, special programmes for youth when in transition from school to work, labour market programmes to provide or promote employment for unemployed and other persons (excluding young and disabled persons) and special programmes for the disabled. For more detailed information regarding the categorization of expenditure on ALMP, see Annex II.1.3. Examples of programmes include:

- "484.10.6.0.1.1 National employment service (SNE) (Servicio nacional de empleo)" in Mexico

- "40.10.6.0.2.5 Support for training in institutions" in Austria

- “620.10.6.0.3.1 Employment-training rotation program” in Portugal

- "300.10.6.0.4.17 Programme for the subsidised employment of special social groups" in Greece

- “554.10.6.0.5.9 Vocational activities/community participation” in New Zealand

- 7. Unemployment - includes all cash expenditure to people compensating for unemployment. This includes redundancy payments to people who have been dismissed through no fault of their own by an enterprise that is ceasing or cutting down its activities out of public resources as well as pensions to beneficiaries before they reach the 'standard' pensionable age if these payments are made because they are out of work or otherwise for reasons of labour market policy. Examples of programmes include:

- "36.10.7.1.1.2 Newstart allowance" for Australian unemployed entitled to an out-of-work unemployment benefit

- “380.10.7.1.2.1 Early retirement for labour market reasons" from National Social Security Institute in Italy.

- $\quad$ 8. Housing - spending items recorded under this heading include rent subsidies and other benefits to the individual to help with housing costs. This includes direct public subsidies to tenants (in some countries, e.g., Norway, homeowners living in their house) 'earmarked' for support with the cost of housing. Because the benefits included here concern earmarked cash payments, by convention they are classified as in-kind benefits (SNA, 1993 - see D.6331). SOCX also reports direct in-kind housing provisions to the elderly and disabled and shelter for those in immediate need in other sections (1.2.1,3.2.1, and 9.2.2, respectively).

Other forms of housing support such as mortgage relief, capital subsidies towards construction and implicit subsidies towards accommodation costs housing can be of a social nature, particularly when such accommodation directly benefits low-income households. However, there is no cross-national agreement on a methodology on coverage and measurement of such support, so that at present, such housing support is not included in SOCX. Nevertheless, such support can be considerable. 
For example, in the Netherlands, the budgetary costs of favourable tax treatment of interest payments and other mortgage costs amounted to almost EUR 12 billion in 2006. Accounting for reduced taxation of private equity in housing (EUR 7.5 billion), income and acquisition tax (EUR 5 billion) as well as municipal rates (EUR 2.25 billion), the net budgetary subsidy to private home ownership in the Netherlands was estimated to be around $2.3 \%$ of GDP in 2006 (Koning et al., 2006). SOCX also does not include (capital-) subsidies towards the construction of housing support, for example in the United States, in 2003 credit for lowincome investment was worth USD 6.2 billion or $0.06 \%$ of GDP (OMB, 2009). SOCX also does not include the value of implicit subsidies towards the cost of housing. For example, in France, almost 5 million households in public social housing pay a lower rent than households in accommodation with similar characteristics in the private rental sector (Ministère de l'Écologie, du Développement et de l'Aménagement durables de la France, 2007). The value of implicit subsidies per household (i.e., the difference between the low rent effectively paid and the rent paid on the market for a dwelling with similar characteristics) is likely to be considerable. However, estimates on the total value of implicit housing subsidies are not available.

- 9. Other social policy areas - includes social expenditure (both in cash and in kind) for those people who for various reasons fall outside the scope of the relevant programme covering a particular contingency, or if this other benefit is insufficient to meet their needs. Social expenditure related to immigrants/refugees and indigenous people are separately recorded in this category. Finally, any social expenditure which is not attributable to other categories is included in the sub-category other.

- "276.10.9.1.1.1 Income support (Social assistance)" in Germany

_ "840.10.9.1.1.1 Earned income tax credit: refundable part (EITC)" in the United States.

\section{II.3.2. Accounting conventions and practices}

\section{Reference, fiscal and tax years}

89. The recording period with respect to the social expenditure data is not the same for each country. Most countries report data by calendar year (1 January to 31 December), except for Australia, Canada, Japan, New Zealand, the United Kingdom and the United States, where the data reported pertain to a financial year which differs from the calendar year. Adopting the same convention as for national accounts, year " $n$ " is taken to mean the year in which a financial year begins, whether it starts on 1 January, 1 April, 1 July or 1 October. In cases where the financial year for social expenditure does not coincide with the calendar year, the relevant periods have been taken on a prorata temporis basis when using GDP (available for calendar years) and the GDP deflator, see below. For all other countries, GDP data refer to the calendar year.

- In Canada, Japan and the United Kingdom, the financial year (n) runs from 1 April (n) to 31 March ( $n+1)$ for social expenditure, requiring an adjustment for GDP (" $n$ ") $=0.75 *$ GDP(n) + $0.25^{*} \mathrm{GDP}(\mathrm{n}+1)$.

- In the United States, the financial year (n) runs from 1 October (n-1) to 30 September (n) for social expenditure, requiring an adjustment for GDP ("n") $=0.25 *$ GDP(n-1) + 0.75GDP(n).

- In Australia and New Zealand, the reference years for social expenditure, although defined as July to June and not by calendar year, correspond to the calculation period for GDP. 
Consequently no special adjustments are required. All the data refer to fiscal years beginning on the $1^{\text {st }}$ July of the year indicated.

SOCX does not include administrative costs

90. SOCX generally excludes administration costs, i.e., the costs incurred with the provision of benefits, as these expenditures do not go directly to the beneficiary. Administration costs cover expenditure on the general overheads of a social expenditure programme: registration of beneficiaries, administration of benefits, collection of contributions, controls, inspection, evaluation and reinsurance.

91. However, regarding the provision of services such as under Active Labour Market Programmes (ALMP), childcare services and public expenditure on health, the administration costs are included in the totals. It should be noted that these data sources include the OECD Education database, the OECD Labour Market Policy database and OECD Health Data, which have their own concepts and definitions. The inclusion of the administrative costs as well as wages for medical staff, employment service staff and childcare workers in the expenditures is justified as they are an integral part of the service being provided to beneficiaries, such as job-seeker reception and counselling, care and education of children, and/or patient reception and hospital services.

SOCX includes capital transfers and records transactions on an accrual basis

92. In line with SNA93, capital investment (i.e., construction costs) are included on an accruals basis, that is if construction costs for a long term-care institution (or hospital) cost USD 1 million (interests included), built over four years, annual reimbursements of USD 250000 would be included each year as investment spending.

SOCX generally excludes loans

93. "The conventional definition of social protection stipulates that the intervention does not involve a simultaneous reciprocal arrangement. This should be conceived as excluding from the scope of social protection any intervention where the recipient is obliged to provide simultaneously something of equivalent value in exchange. For instance, interest-bearing loans granted to households are not social protection because the borrower commits himself to paying interest and to refund the capital sum. Still, if the loan is interest-free or granted at an interest rate well below the current market rate for social protection reasons, the amount of interest waived qualifies as a social benefit" (Eurostat, 2008).

\section{II.3.3. Data sources}

94. The nature of SOCX data-processing is not straightforward as data do not derive from one allencompassing questionnaire, but are taken from different sources in different formats:

- For all $O E C D$ countries data on public expenditure on health and public expenditure on active labour market policies (ALMPs) are taken from the OECD Health Data and the OECD database on Labour Market Programmes, respectively (OECD, 2010d, and 2011b, Statistical Annex). Data on education of 3,4 and 5 year olds (ISCED 0) as in the OECD Education database feeds into the series on social spending on early care and education services. Data on unemployment compensation (cash transfers) are taken from the LMP database for OECD countries that do not belong to the EU and from ESSPROS for EU countries. 
- For 10 non-European OECD countries, data delivered through the services of the delegates to the Working party on Social Policy of the Employment, Labour and Social Affairs committee responding to the SOCX Questionnaire.

- For 24 European countries (EU-21, Iceland, Norway and Switzerland), data on social expenditure is provided by EUROSTAT as based on the information in their ESSPROS database (EUROSTAT, 2010).

95. This is not an ideal way to collect data, not least because it limits interaction with data producers in European OECD countries. However, there has been little choice in the matter. From the start, OECD member states that also belong to the EU have insisted on providing data to the OECD via EUROSTAT in order to avoid having to deal with multiple social spending questionnaires. This is understandable, but does mean that a) information is only received from EUROSTAT once it has 'validated' the data for individual countries and b) data received in ESSPROS format has to be made compatible with information for nonEuropean OECD countries. Furthermore, as ESSPROS data do not include all public spending on health and/or spending on active labour market policies, all individual country files are inevitably built from different sources. ${ }^{14}$

96. To achieve comparability of spending data for all OECD countries involves going through the EUROSTAT data submission to identify and siphon-out voluntary private social expenditure items to ensure compatibility with the public (and mandatory private) spending data for all OECD countries, and more generally ensure consistency of the spending data that are taken from different sources. Annex II.1 includes more detail on data sources.

97. Other reference series used in SOCX are from OECD (2011e):

- Gross Domestic Product (GDP)

- Deflator for GDP

- Gross Domestic Product at 2000 prices (GDPV)

- Gross National Income (GNI)

- Net National Income (NNI)

- Total General Government expenditure (GOV)

- Purchase Power Parities (PPP)

- Exchange rate (EXC)

- $\quad$ Population (POP)

14 The 'core system of ESSPROS' focuses on support that can be 'allocated' to individuals and, consequently, it does not include all spending on public health expenditures or active labour market programmes. For example, ESSPROS does not include spending on investment in medical facilities, preventive health initiatives as anti-smoking campaigns, and health education and training more generally. 


\section{II.4. Net (after tax) social expenditure}

98. Gross social spending data as for example presented in public budgets, do not account for how tax systems affect public and private spending on social protection. And as this effect can be considerable and varies across countries, it affects cross-national comparisons of social expenditure.

99. General tax revenue is used to finance public social spending, and sometimes revenue streams (i.e., social security contributions) are earmarked for that purpose (OECD, 2010a). However, tax systems also affect levels of social expenditure, and broadly speaking they do so in three ways ${ }^{15}$ :

1. Direct taxation of benefit income: Governments levy income tax and social security contributions on cash transfers to beneficiaries, in which case redistribution of resources is lower than suggested by gross spending indicators.

2. Indirect taxation of consumption by benefit recipients: Benefit income is provided to finance consumption of goods and services. Indirect taxes reduce the consumption which can be financed out of a given level of benefit income.

3. Tax breaks for social purposes: Governments also make use of the tax system to directly pursue social policy goals. Fiscal measures with social effects are those which can be seen as replacing cash benefits (e.g., child tax allowances) or stimulating the provision of private benefits (e.g., tax relief towards the provision of private health plans). These tax breaks for social purposes (TBSPs) can be directly awarded to households, but also include tax relief for employers and private funds that ultimately benefit households (e.g., favourable tax treatment of employerbenefits provided to households, favourable tax treatment of private funds).

100. The adjustments for direct and indirect taxation of benefits do not affect service spending, even though such services, e.g., pharmaceutical products, can be subject to indirect taxation. Data on spending on social services that are subject to indirect taxation and at what rate is not available on a comprehensive basis.

\section{II.4.1. Direct taxation of cash benefits}

101. In some OECD countries benefits are taxed in the same way as earnings, while in other countries most benefits are taxed at a reduced rate. Yet in other countries, almost all benefits are paid net of direct taxation. Treatment of unemployment insurance benefits varies considerably across countries (Table II.3). For example, in Austria the recipient of an unemployment benefit who previously had earnings equivalent to average earnings and who lived in a couple-family with two young children received the equivalent of EUR 15812 in 2007, on which he or she did get a tax credit of EUR 669. By contrast, a similar person in Sweden received annual income support of EUR 19112 but paid EUR 5147 in income taxes and socialsecurity contributions so that net benefit income was EUR 13 966. Thus, net income for such a family in Sweden is lower than in Austria, although gross income was much higher. In aggregate spending terms, this means that countries that tax transfer incomes rather heavily divert a significant part of transferred income to flow back into the coffers of the Treasury. As a result, net (after tax) public spending on unemployment benefits is about $75 \%$ of the level suggested by gross indicators in Sweden.

15 These fiscal adjustments measure 'first round effects' concerning the net value of benefits. Hence, direct taxation of the earnings of those who provide services (e.g., staff in hospitals or childcare centres) is not included in the calculations. 
102. Moreover there are considerable differences between how different types of benefits are being taxed (Table II.3). In general, unemployment assistance, social assistance and housing benefits and family benefits are generally not taxed. In contrast, public and private retirement and disability pension payments are generally taxed, but frequently at reduced rates $(\mathrm{OECD}, 2011 \mathrm{c})$, while continued wage payments in case of absence due to sickness are taxed as earnings (OECD, 2011d).

Table II.3: Tax treatment of benefits differs across countries

Tax and social security treatment of benefits in 2007

\begin{tabular}{|c|c|c|c|c|c|}
\hline & $\begin{array}{l}\text { Pension transfers (old- } \\
\text { age, disability) }\end{array}$ & Child benefits & Unemployment & Housing & Social assistance \\
\hline Australia & $\mathrm{T}$ (reduced) & $\mathrm{N}$ & $\mathrm{T}(\mathrm{n}) \mathrm{S}(\mathrm{n})$ & $\mathrm{N}$ & -- \\
\hline Austria & TS(reduced) & $\mathrm{N}$ & $*$ & $\mathrm{~N}$ & $\mathrm{~N}$ \\
\hline Belgium & T(reduced)S(reduced) & $\mathrm{N}$ & $\mathrm{T}(\mathrm{n})$ & -- & $\mathrm{N}$ \\
\hline Canada & T(reduced) & -- & $\mathrm{T}$ & -- & $\mathrm{N}$ \\
\hline Czech Republic & $\mathrm{T}$ (reduced) & $\mathrm{N}$ & $\mathrm{N}$ & $\mathrm{N}$ & $\mathrm{N}$ \\
\hline Denmark & $\mathrm{T}$ & $\mathrm{N}$ & TS(reduced) & $\mathrm{N}$ & TS(reduced) \\
\hline Finland & $\mathrm{T}$ (reduced)S(reduced) & $\mathrm{N}$ & TS(reduced) & $\mathrm{N}$ & $\mathrm{N}$ \\
\hline France & TS(reduced) & $\mathrm{N}$ & TS(reduced) & $\mathrm{N}$ & $\mathrm{N}$ \\
\hline Germany & $\mathrm{T}$ (reduced)S(reduced) & tc & $*$ & $\mathrm{~N}$ & -- \\
\hline Greece & TS(reduced) & $\mathrm{N}$ & $\mathrm{N}$ & $\mathrm{N}$ & -- \\
\hline Hungary & $\mathrm{T}$ & $\mathrm{N}$ & TS(reduced) & $\mathrm{N}$ & $\mathrm{N}$ \\
\hline Iceland & $\mathrm{T}$ & $\mathrm{N}$ & TS & $\mathrm{N}$ & $\mathrm{TS}$ \\
\hline Ireland & TS(reduced) & $\mathrm{N}$ & $\mathrm{T}(\mathrm{n})$ & $\mathrm{N}$ & $\mathrm{N}$ \\
\hline Italy & $\mathrm{T}($ reduced $)$ & $\mathrm{N}$ & TS(reduced) & -- & -- \\
\hline Japan & TS(reduced) & $\mathrm{N}$ & $\mathrm{N}$ & -- & $\mathrm{N}$ \\
\hline Korea & $\mathrm{T}$ (reduced) & -- & $\mathrm{N}$ & -- & $\mathrm{N}$ \\
\hline Luxembourg & TS(reduced) & $\mathrm{N}$ & TS(reduced) & TS & $\mathrm{TS}$ \\
\hline Mexico & $\mathrm{T}(\mathrm{n})$ & $\mathrm{N}$ & -- & -- & -- \\
\hline Netherlands & TS(reduced) & $\mathrm{N}$ & $\mathrm{TS}$ & $\mathrm{N}$ & * \\
\hline New Zealand & $\mathrm{T}$ & $\mathrm{N}$ & -- & $\mathrm{N}$ & -- \\
\hline Norway & $\mathrm{T}($ reduced)S(reduced) & $\mathrm{N}$ & $\mathrm{TS}$ & $\mathrm{N}$ & $\mathrm{N}$ \\
\hline Poland & TS(reduced) & $\mathrm{N}$ & $\mathrm{T}$ & $\mathrm{N}$ & $\mathrm{N}$ \\
\hline Portugal & $\mathrm{T}$ & $\mathrm{N}$ & $\mathrm{N}$ & -- & $\mathrm{N}$ \\
\hline Slovak Republic & $\mathrm{T}$ (reduced) & $\mathrm{N}$ & $\mathrm{N}$ & -- & $\mathrm{N}$ \\
\hline Spain & $\mathrm{T}$ (reduced) & $\mathrm{N}$ & TS(reduced) & -- & $\mathrm{T}(\mathrm{n})$ \\
\hline Sweden & $\mathrm{T}$ & $\mathrm{N}$ & $\mathrm{TS}$ & $\mathrm{N}$ & $\mathrm{N}$ \\
\hline Switzerland & $\mathrm{T}$ & $\mathrm{T}$ & TS(reduced) & -- & $\mathrm{N}$ \\
\hline Turkey & $\mathrm{N}$ & -- & $\mathrm{N}$ & -- & -- \\
\hline United Kingdom & $\mathrm{T}$ & $\mathrm{N}$ & $\mathrm{T}(\mathrm{n}) \mathrm{S}(\mathrm{n})$ & $\mathrm{N}$ & $\mathrm{N}$ \\
\hline United States & $\mathrm{T}$ (reduced) & $\mathrm{N}$ & $\mathrm{T}$ & $\mathrm{N}$ & $\mathrm{N}$ \\
\hline
\end{tabular}

.Notes: T: Taxes are payable, S: Social security contributions (SSC) are payable, N: Neither taxes nor SSC are levied, T(n) or S(n): (Long-term) recipients will not pay the taxes or SSC as the credits, allowances or zero rate bands exceed the benefit level.

--. No specific scheme or no information available, "(reduced)": A reduced rate is payable for beneficiaries, *.:Benefit is a proportion of after tax income (and thus not taxable), tc: Non-wastable tax credit.

Source: Sources: OECD Tax-Benefit models database (www.oecd.org/els/social/workincentives); OECD (2011c), Pensions at a Glance. 


\section{II.4.1.1. Methods and sources; administrative records, microsimulation and microdata}

103. Broadly speaking, there are two ways to adjust gross spending items (e.g., spending on unemployment compensation or old-age cash benefits) for the impact of direct taxation. Sometimes, national sources provide concrete information on the value of tax paid on a particular (set of) benefit(s). Such information is the most reliable source, and is based on data from tax offices and/or social insurance funds for social security contributions. However, such information is rare, and is restricted to information on payments of social security contributions by benefit recipients in Germany and Spain. In some other countries (Austria, Czech Republic, France, Italy, Ireland and Portugal), the adjustment for direct taxation on cash benefits has been calculated on basis of estimates of tax paid by benefit recipients (over some items) based on administrative sources, including tax statistics (see Annex I.2). For Belgium the amounts of tax and social security contributions paid on benefit income are based on the national tax statistics and national accounts, respectively.

104. For other countries the magnitude of direct tax paid by benefit recipients was determined while using estimates supplied by national sources on 'average itemised tax rates' (AITR): e.g., the average tax rate (including social security contributions) on a particular spending item, e.g., public pension benefit, unemployment compensation or parental leave payments. These AITRs were estimated on the basis of a variety of national sources including: administrative data on the basis of tax records (France, Iceland, Japan and the United States). Otherwise 'microsimulation-models' and micro data sets were used to generate itemised tax rates. Such information underlies the estimates of direct taxation of benefits in Australia, Canada, Denmark, Finland, Korea, the Netherlands, New Zealand, Norway, Sweden and the Uinted Kingdom. Subsequently, these AITRs were applied to gross social spending items as recorded in the database.

105. Countries where almost all benefit income is taxable and that use microsimulation models and microdata sets to estimate AITRs generally report such information at the greatest level of detail and have the greatest number of AITRs for different transfer items, e.g., Denmark reports AITRs for 21 different transfer items and Sweden for 10. Countries that have only a few taxable benefits, and (therefore) base their estimated AITR on administrative information (as related to the level of detail on the income tax form) report only a few different tax rates.

Estimating Average Itemised Tax Rates through 'Microsimulation'

106. The concept of AITRs has been developed to facilitate identification of different tax levies on different social benefits. The AITR can be defined as the total taxes paid by those receiving a given benefit, divided by the total income (from all sources) of those receiving that benefit. Formally, the relevant calculations are:

$$
\operatorname{AITR}_{\mathrm{i}}=\quad \Sigma_{\mathrm{tu}=1, \mathrm{n}} \mathrm{TI}_{\mathrm{i}} / \Sigma_{\mathrm{tu}=1, \mathrm{n}} \mathrm{I}_{\mathrm{i}}
$$

where: I is the amount of taxable income-type "i", and TI is the amount of tax paid on that particular amount of income, "i" is the type or category of income, "tu" is a tax unit with income-type "i", and " $n$ " is the number of tax units in the sample with income of type "i". The broad income categories "i" include old-age cash benefits, unemployment compensation, wage income, etc. (see table Q3).

107. Microsimulation-models and micro-data sets contain detailed information on both the incomes received by households and their taxation. Microsimulation techniques generate reliable estimates, but estimation procedures require assumptions on the way income is allocated. Here it is assumed that if a benefit is non-taxable, as are many child payments, then the relevant AITR is a priori considered to be equal to zero. If transfer income is the only income received, the average tax rate (including social security contributions) on this income can be used to calculate net transfer income. However, the calculation of 
direct taxation of benefit income is more complicated when different types of income are involved; people who receive either different benefits during a year, or whose annual income is a combination of earnings with, say, unemployment benefits, or a combination of transfers from different pension plans. In this situation it is necessary to allocate taxes paid to the various income-components, and it is assumed that the tax due is divided over the different income components according to the weight of each type of income. Hence, if benefits provide $75 \%$ of annual income and earnings $25 \%, 75 \%$ of total income tax is assumed to be paid on benefit income (For some aspects of taxation (e.g., deductible expenses related to work), there is a direct link between the income component and taxation. In these cases it is preferable to allocate such deductions only to the relevant income component).

108. Furthermore, benefit income can be subject to a progressive tax schedule (possibly applied to the total of several income sources). In order to avoid an ordering of different parts of income and arbitrary decisions on what part of household income should be taxed at the higher or the lower rate, the average itemised tax rate should be calculated on the basis of the tax rates that households face over a particular income (or group of incomes). Allocating income tax paid according to the relative weight of the different income components (see above) and grossing up for the households in the sample, AITRs can be calculated (Box II.4).

\section{Box II.4: An example of calculating Average Itemised Tax rates}

It is straightforward to calculate average itemised tax rates (AITRs) on benefit income if households have only one source of income. For example, if a retiree receives a public pension payment worth 100 units per annum at a 'standard' tax rate of $10 \%$, net annual transfer income is 90 units. If, in addition, all households in receipt of public retirement income had no income from other sources, the AITR on public pension income would be $10 \%$. If among the retirees some were to receive non-taxable child supplements, this income would be disregarded for the calculation of the tax rate on his/her household income, while the AITR on child supplements would be nil.

Often pensioners receive income from different sources. Consider the case of a retiree who receives a public pension worth 50 units and a private pension worth 100 units. In the absence of progressivity in the tax system, the household tax rate would remain 10\%, and net transfer income would be 135 units. However, a substantial increase of income may well lead to parts of incomes being taxed at a higher rate (see household 4 in Table Box 4.1), so that the 'average' tax rate increases. In this case 100 units of transfer income are taxed at $10 \%$ and 50 units are taxed at a rate of $15 \%$. Total income tax is worth 17.5 units, which is allocated over public and private pension income components according to their relative weight in total household income (Table Box 4.1). Thus, the methodology does not imply an ordering of different parts of income, whereby different income sources are taxed differently according to an arbitrary decision on which part of income should be taxed at higher or lower tax rates. Differences in AITRs are associated with income groupings wherein benefit recipients typically find themselves.

Table Box II.4: Calculating AITRs on two types of income

\begin{tabular}{|c|c|c|c|c|c|c|c|}
\hline \multirow[t]{2}{*}{ Household } & \multirow[t]{2}{*}{$\begin{array}{l}\text { Public } \\
\text { pension }\end{array}$} & \multirow[t]{2}{*}{$\begin{array}{l}\text { Private } \\
\text { pension }\end{array}$} & \multirow[t]{2}{*}{$\begin{array}{l}\text { Total } \\
\text { household } \\
\text { income }\end{array}$} & \multirow[t]{2}{*}{$\begin{array}{l}\text { Income } \\
\text { tax rate }\end{array}$} & \multirow[t]{2}{*}{$\begin{array}{l}\text { Tax } \\
\text { paid }\end{array}$} & \multicolumn{2}{|c|}{$\begin{array}{l}\text { Allocation of tax over } \\
\text { pension income } \\
\text { components }\end{array}$} \\
\hline & & & & & & Public & Private \\
\hline 1 & 50 & 25 & 75 & $10 \%$ & 7.5 & 5.0 & 2.5 \\
\hline 2 & 75 & 50 & 125 & $15 \%$ & 13.8 & 8.3 & 5.5 \\
\hline 3 & 100 & 0 & 100 & $10 \%$ & 10.0 & 10.0 & 0.0 \\
\hline 4 & 50 & 100 & 150 & $15 \%$ & 17.5 & 5.8 & 11.7 \\
\hline 5 & 50 & 250 & 300 & $15 \%$ & 40.0 & 6.7 & 33.3 \\
\hline Total & 325 & 425 & 750 & & 88.8 & 35.8 & 53 \\
\hline \multirow{2}{*}{\multicolumn{6}{|c|}{$\begin{array}{l}\text { AITR public pension income }=\text { tax paid over public pension/total public pension } \\
\text { income } \\
\text { AITR private pension income }=\text { tax paid over private pension/total private pension } \\
\text { income }\end{array}$}} & $11.1 \%$ & \\
\hline & & & & & & & $12.5 \%$ \\
\hline
\end{tabular}


109. As already noted, if benefit income of a particular type is non-taxable, then the relevant AITR is a priori equal to zero. However, it is possible that income derived from non-taxable benefits affects direct taxation of taxable benefit income in an indirect manner, as it is considered in the income-test of other benefit programmes, so receipt of non-taxable benefits may reduce the amount of other income transfers households may receive. In Canada, three social programmes (guaranteed income supplement, (provincial) social assistance, and workers compensation) affect the calculation of taxation of benefits in this manner. These three benefit payments are non-taxable, but relevant income is considered in the income-test for other benefits, and thus reduces payments under other benefit programmes to these recipients. In order to take this indirect effect into account, the Canadian authorities removed these three programmes as sources of income from their simulations to calculate an average (marginal) tax rate. This rate was applied to each of these three social transfers to determine the implicit tax paid, which was then divided by the amount of transfer spending for the three items to find the AITRs (see Annex I.2).

\section{II.4.2. Indirect taxation of consumption out of benefit income}

110. Consumption taxes reduce the real value of consumption which can be financed out of a given level of benefits, and (as with direct taxation of benefit income) establish another flow back in tax receipts to the government. ${ }^{16}$ Similarly to differences in direct taxation of benefit income, cross-country differences in indirect taxation affect comparisons of welfare state spending. In countries where indirect taxation is relatively limited (i.e., in non-European OECD countries), gross spending levels can also be relatively low to generate the same net income level for benefit recipients in countries with high indirect tax rates. For example, in order to provide benefit recipients with a net income of 100 units, a country like the United States with an average indirect tax rate of close to $5 \%$ needs to pay a gross benefit of about 106 units. In Denmark, where the average indirect tax rate is about $25 \%$, a gross payment would have to be around 133 units to have an equivalent net value. To some extent the relatively low social spending to GDP ratios in the United States and in other non-European OECD countries are related to the low indirect tax levels that prevail in these countries, and accounting for this feature improves the quality of cross-country comparisons of social spending.

111. In some countries, policy explicitly recognises the impact of indirect taxation on the financial position of low-income households (many of whom receive transfer income). For example, when the Goods and Services Tax was introduced in Australia in July 2000 at a rate of $10 \%$ (with food being exempt), a compensation package for social protection benefit recipients was introduced at the same time. Similarly, Canada has a Goods and Services Tax rebate to support low-income households.

\section{II.4.2.1. Methods and sources: national accounts and revenue statistics}

112. Detailed information on consumption by benefit recipients is not available. Alternatively, household expenditure surveys allow for the analysis of different spending patterns across different income groups, but such information is not readily available for all countries on a comprehensive basis. Moreover, the results of such surveys suggest that indirect tax payments are under-reported as estimates of aggregate tax receipts on the basis of such surveys is well below actual tax receipts, see for example, Gho et al., (2010). Therefore, the approach followed here is to calculate an average implicit indirect tax rate based on aggregate data available for all countries as in the OECD Revenue Statistics and the OECD National Accounts (OECD, 2010a, and 2010e). This approach, while approximate, is clear and transparent.

16 The chosen methodology might be criticised for implicitly assuming that benefit recipients do not save but consume all their benefit income. Savings are, presumably, consumed at some point, and in any case the marginal propensity to consume out of benefit income is likely close to 1 , limiting the scope for error. 
113. Table II.4 contains three possible measures of indirect taxes. The first, as presented in line 3, captures the amount of indirect tax receipts through general consumption taxes and excise duties charged on particular goods. Line 4 in Table II.4 includes these taxes as well as profits from fiscal monopolies, customs duties, taxes on services, and some other minor taxes. Line 5 adds additional taxes on the use of goods, such as licenses for motor vehicles and for the sale of alcohol. A case could be made for using any of these measures of indirect taxation, but the indirect tax concepts reflected in lines 4 and 5 of Table II. 4 include more items of indirect taxation that are not paid by the household sector than reflected in line 3 (and even this relatively limited measure includes some taxes not paid by the household sector). The indirect tax measure in line 3 of Table II. 4 includes the smallest margin of error, and is thus the most appropriate to use for calculating indirect taxes paid on consumption out of benefit income.

114. Private consumption as in the National Accounts is given in line 1 of Table II.4. However, the OECD Revenue Statistics includes tax revenue collected by government from itself. For example, if one part of government purchases some goods and services, it may be charged indirect tax (which constitutes a tax flow within the government sector). To reflect this, government consumption expenditure is added to private consumption expenditure while subtracting that part of government consumption which consists of compensation of employees (line 2, Table II.4). In this manner, a consistent approximation of the tax base of indirect taxes is found.

115. The average implicit indirect tax rate is then the ratio of revenue from general consumption taxes plus excise duties to a broad consumption tax base, i.e., private consumption and government consumption minus government wages - line 6, in Table II.4. In 2007, the implicit average indirect tax rates were lowest in the United States (4.1\%), Mexico (6.0\%) and Japan (6.3\%) and were around $10 \%$ in Australia and Canada. Indirect tax rates ranged from $12-14 \%$ in Germany, Korea, Italy and Spain and ranged from $15 \%$ to $23.7 \%$ in most European countries, and were at $26 \%$ in Denmark. 
DELSA/ELSA/WD/SEM(2011)9

\section{Table II.4: Average implicit indirect tax rates of consumption out of benefit income}

Indirect taxes paid out of consumption of cash transfers, in millions of national currency, in 2007

\begin{tabular}{|c|c|c|c|c|c|c|c|c|c|c|c|c|c|c|c|c|c|c|c|c|c|c|c|c|c|c|c|c|}
\hline & AUS & AUT & BEL & CAN & C正 & DNK & $F \mathbb{N}$ & FRA & DEU & ISL & IRL & TTA & JPN & KOR & LUX & MEX & NLD & $\mathrm{NZZ}$ & NOR & POL & PRT & SVK & ESP & SWE & TUR & GBR & USA & OECD-27 \\
\hline (1) Private final consumption expenditure & 655287 & 143812 & 170965 & 5851603 & 1686837 & 821664 & 90708 & 1074170 & 1378940 & 751598 & 88882 & 907546 & 292523200 & 530264100 & 11826 & 7316008 & 264099 & 105515 & 940067 & 711872 & 110635 & 34457 & 604022 & 1460162 & 601239 & 892990 & 9826400 & - \\
\hline $\begin{array}{l}\text { (2) Private consumption plus Govemment } \\
\text { consumption minus Govemment wages }\end{array}$ & 772490 & ) 168524 & 206442 & 2969399 & 2136460 & 976555 & 106090 & 1268834 & 1645440 & 874843 & 100215 & 1047738 & 353089300 & 602100500 & 14733 & 7575007 & 355618 & 122381 & 1107317 & 809734 & 119920 & 40914 & 689661 & 17911632 & 709055 & 1034132 & 10638800 & - \\
\hline $\begin{array}{l}\text { (3) General consumption taxes plus excise } \\
\text { duties }(5110+5121)^{2}\end{array}$ & 69843 & 27634 & 31191 & 93475 & 365780 & 254214 & 21070 & 183323 & 234198 & 183485 & 20085 & 128527 & 22214700 & 68822000 & 3484 & 456021 & 59857 & 16676 & 260555 & 1452092 & 20274 & 6318 & 86147 & 371028 & 82396 & 132656 & 437366 & - \\
\hline 5110 General taxes & 45486 & 20988 & 23931 & 70385 & 232288 & 175426 & 15054 & 139884 & 170387 & 137593 & 14156 & 95623 & 12841100 & 40942000 & 2090 & 409013 & 42216 & 15046 & 189401 & 96152 & 14339 & 4147 & 63400 & 286211 & 43285 & 92043 & 300061 & - \\
\hline (4) Taxes on production sale transfer (5100) & 85023 & 29716 & 34506 & 113753 & 365998 & 260896 & 22605 & 197714 & 247858 & 191957 & 20211 & 152930 & 23240700 & 78414000 & 3546 & 1042372 & 60742 & 18835 & 265620 & 1483662 & 21862 & 6471 & 92785 & 380990 & 92605 & 141358 & 534202 & - \\
\hline (5) Taxes on Goods and Services (5000) & 92872 & 31574 & 36957 & 121925 & 393042 & 275763 & 23242 & 203104 & 257019 & 214988 & 21185 & 169311 & 26255800 & 80861000 & 3614 & 1063583 & 63603 & 20301 & 281797 & 156400 & 22284 & 6948 & 99653 & 394073 & 96766 & 147324 & 644821 & - \\
\hline \multicolumn{29}{|c|}{ Implicit average indirect tax rate on consumption out of benefit income } \\
\hline $\begin{array}{l}\text { (6) Using general consumption taxes } \\
\text { plus excise duties (3)/(2) }\end{array}$ & $9.0 \%$ & $16.4 \%$ & $15.1 \%$ & $69.6 \%$ & $17.1 \%$ & $26.0 \%$ & $19.9 \%$ & $14.4 \%$ & $14.2 \%$ & $21.0 \%$ & $20.0 \%$ & $12.3 \%$ & $6.3 \%$ & $11.4 \%$ & $23.7 \%$ & $6.0 \%$ & $16.8 \%$ & $13.6 \%$ & $23.5 \%$ & $17.9 \%$ & $16.9 \%$ & $15.4 \%$ & $12.5 \%$ & $20.7 \%$ & $11.6 \%$ & $12.8 \%$ & $4.1 \%$ & $15.1 \%$ \\
\hline $\begin{array}{l}\text { (7) using a broad concept of the indirect } \\
\text { tax base (5)/(2) }\end{array}$ & $12.0 \%$ & $18.7 \%$ & $17.9 \%$ & $12.6 \%$ & $18.4 \%$ & $28.2 \%$ & $21.9 \%$ & $16.0 \%$ & $15.6 \%$ & $24.6 \%$ & $21.1 \%$ & $16.2 \%$ & $7.4 \%$ & $13.4 \%$ & $24.5 \%$ & $14.041 \%$ & $17.9 \%$ & $16.6 \%$ & $25.4 \%$ & $19.3 \% 1$ & $18.6 \%$ & $17.0 \%$ & $14.4 \%$ & $22.0 \%$ & $13.6 \%$ & $14.2 \%$ & $6.1 \%$ & $17.3 \%$ \\
\hline $\begin{array}{l}\text { using a bradd concept of the indirect } \\
\text { (8) tax base and ignoing govermment } \\
\text { consumpion (5)/(1) }\end{array}$ & $14.2 \%$ & $22.0 \%$ & $21.6 \%$ & $14.3 \%$ & $23.3 \%$ & $33.6 \%$ & $25.6 \%$ & $18.9 \%$ & $18.6 \%$ & $28.6 \%$ & $23.8 \%$ & $18.7 \%$ & $9.0 \%$ & $15.2 \%$ & $30.6 \%$ & $14.538 \%$ & $24.1 \%$ & $19.2 \%$ & $30.0 \%$ & $22.0 \% 2$ & $20.1 \%$ & $20.2 \%$ & $16.5 \%$ & $27.0 \%$ & $16.1 \%$ & $16.5 \%$ & $6.6 \%$ & $20.4 \%$ \\
\hline $\begin{array}{l}\text { Indirect taxes paid out of consumption of } \\
\text { total cash transfers, in percentage of GDP }\end{array}$ & $0.9 \%$ & $2.8 \%$ & $2.9 \%$ & $1.0 \%$ & $2.1 \%$ & $3.0 \%$ & $2.5 \%$ & $2.5 \%$ & $2.1 \%$ & $2.0 \%$ & $2.0 \%$ & $2.0 \%$ & $0.8 \%$ & $0.3 \%$ & $2.8 \%$ & $0.1 \%$ & $2.3 \%$ & $1.1 \%$ & $2.4 \%$ & $2.3 \%$ & $2.3 \%$ & $1.6 \%$ & $1.5 \%$ & $2.5 \%$ & $0.7 \%$ & $1.9 \%$ & $0.5 \%$ & $1.8 \%$ \\
\hline
\end{tabular}

Notes: a) The 4-digit codes in the second column refer to the categorisation used in the OECD (2010b) Revenue Statistics.

Sources: OECD (2010e), National Accounts (www.oecd.org/statistics/national-accounts), and OECD (2010b), Revenue Statistics, OECD, Paris, (lines 3, 4, and 5). 


\section{II.4.3. Tax breaks for social purposes}

116. Expenditures made through the tax system, or tax expenditures can take different forms: exemptions (income excluded from the tax base); allowances (amounts deducted from gross income); credits (amounts deducted from tax liability); rate reliefs (tax rate reduction for specific groups, e.g., senior citizens); and tax deferrals. However, definitions of 'tax expenditures' vary across countries (OECD, 1996). In particular, there is no international agreement on what constitutes a 'benchmark' tax system which can be used to identify tax expenditures. National benchmarks (the 'normal' structure of the tax system) against which tax expenditures are being measured vary considerably, which hampers the measurement of tax expenditures on a comparable basis across countries. However, that does not rule out a comparison of a sub-group of 'tax expenditures' - such as those related to social protection systems. This is because the approach followed here measures the amount clawed back in taxation over cash transfers and the value of direct support to benefit recipients provided through the tax system, for which reference to a 'benchmark' tax system is not required.

117. Many governments of OECD countries pursue social policy objectives through the tax system. Broadly speaking there are two groups of such measures. One is reduced taxation on particular sources of income or types of household. For example, some cash transfers could be taxed at a zero or reduced rate. This sort of tax relief is equivalent to a variation in direct taxation of benefit income and has already been accounted for in the section on direct taxation (see above). Thus, exemptions of benefits from taxation or reduced rates on benefit income are reflected in the calculations of direct taxation levied on benefit income (e.g., a zero tax rate is applied to spending on child benefits) and are not recorded here again as a Tax Break with a Social Purpose (TBSP) in order to avoid double counting. A tax allowance for dependent children (which is different from non-taxation of child benefits) is recorded as a TBSP (see below).

118. The second group of tax measures with social effects concern Tax Breaks for Social Purposes (TBSPs) and are defined as:

"those reductions, exemptions, deductions or postponements of taxes, which: a) perform the same policy function as transfer payments which, if they existed, would be classified as social expenditures; or $b$ ) are aimed at stimulating private provision of benefits".

119. TBSPs which can be seen as replacing cash benefits often involve tax credits towards dependent children. TBSPs that aim to stimulate the provision of private expenditures include tax relief for noncommercial non-government organisations, tax advantages towards private health insurance contributions, and favourable tax treatment of private pensions.

\section{II.4.3.1. Methods and sources; the valuation of tax revenue forgone}

120. Information on the value of tax breaks with a social purpose can often be found in so-called 'tax expenditure reports' as published by national authorities, for example, Australian Government (2009), Department of Finance Canada (2008), Government of Ireland (2008), and the OMB (2009) for the United States. Such reports generally present estimates on the revenue forgone through tax measures: i.e., the amount by which tax revenue is reduced because of the presence of fiscal measures. Such reports generally cover favourable tax treatment by central/federal governments, but do not account (and neither does this report) for tax assistance by sub-national levels of government, as in for example, Canada, Japan and the 
United States. Comprehensive information across countries is not (yet) available, but the value of subnational TBSPs in Canada could be close to $0.6 \%$ of GDP. ${ }^{17}$

121. Tax expenditure reports in many countries do aggregate different measures to give an overall picture of the importance of tax expenditures. Strictly speaking this causes methodological problems, since tax expenditures and TBSPs are interdependent. For example, consider the combined existence of a tax allowance for sole parents and another separate tax relief towards the cost of childcare. The value of these two fiscal measures would normally be calculated (and presented) separately. However, if one of the two TBSPs were to be eliminated, then some taxpayers may end up in a higher marginal tax rate category, thereby increasing the value of the other TBSP (unless the claimant already received the maximum amount of relief). The value of both schemes considered jointly would be greater than the sum of the separate measures, since each is calculated assuming the other remains in force. Whereas individual revenue forgone estimates overstate the cost of TBSPs (they take no account of behavioural effects which can be expected to reduce (future) tax payments) the aggregate of such estimates understate the overall costs.

122. There are different ways of calculating the value of TBSPs (OECD, 1996). The already mentioned 'revenue forgone' method is an ex post measure of the amount by which tax revenue is reduced because of a particular measure. Table II.5 shows that depending on the measurement technique the estimated value of the tax break can vary significantly. Calculating the present value of favourable treatment of pension plans does not necessarily lead to estimates that are larger than the revenue forgone method that does not account for deferred pension earnings on current contributions or tax paid over benefits in future. While the present value of favourable tax treatment of individual retirement accounts is well below estimates based on the revenue foregone method, the opposite holds for the exclusion of pension contributions. This suggests that participants of individual retirement plans do have very favourable tax treatment on their contributions relative to their future tax payments on relevant income transfers. In fact, participants in individual retirement accounts can choose as to whether they wish to pay tax on current contributions or future payments: it appears that many choose the latter option.

Table II.5: Value of selected tax breaks for pensions, the United States, 2007

\begin{tabular}{lcc}
\hline & $\begin{array}{c}\text { Calculation Method } \\
\text { Present value }\end{array}$ \\
Exclusion of Pension contributions and earnings-employer plans & $\begin{array}{r}\text { Revenue Forgone } \\
\text { Value (in million US dollars) }\end{array}$ \\
\cline { 2 - 2 } Exclusion of contributions and earnings for Individual Retirement Accounts & 52470 & 74120 \\
Exclusion of contributions and earnings for Keogh Plans & 5970 & 8600 \\
\hline
\end{tabular}

Note: The Administration has dropped the estimates of the outlay equivalents because they were often the same as the normal tax expenditure estimates, and the criteria for applying the concepts as to when they should differ were often judgmental and hard to apply with consistency across time and across tax expenditure items.

Source: US OMB (2009), Analytical Perspectives, Budget of the United States Government, fiscal year 2009

17 In Canada a crude estimate of direct taxation of benefit income of both federal and provincial taxes assumes that provincial taxes were about $50 \%$ of federal taxes in the mid-2000s. Using the latter as a ruleof-thumb the real value of TBSPs similar to cash benefits may well have been around 2\% of GDP at factor cost in 2007 rather than $1.3 \%$ of GDP - the value of Federal TBSPs. A small part of provincial tax reductions are recorded here, for social objectives (family size, presence of children, dependants, etc). 
123. Social expenditure and TBSPs can both be calculated on a cash or on an accruals basis. The former approach estimates the effect on government cash flows, the latter on the tax liabilities accruing to government in a particular period. Except for TBSPs for pensions, there is likely to be little difference between estimates calculated on these two bases. ${ }^{18}$ Favourable tax treatment of funded pension payments also has to account for the effect that tax treatment of current pension contributions may have on future tax payments. For example, a pension contribution in 2007 would cause a deferral of tax-payments on wages in 2007 and on pension earnings on this contribution (e.g., interest, capital gains) in later years. However, in some future year the 2007 pension contribution and accrued earnings will be paid out and taxes will be due: these receipts are included in the present value estimate.

124. Tax breaks for pensions include tax exemptions for contributions to private pensions, and tax relief for investment income of capitalised pension funds. Because of the complexities of calculating the value of these tax reliefs that are given at various stages of what is a form of contractual savings, there is no comparable data set available on the value of tax breaks for pensions across countries. Therefore, a comprehensive analysis of Tax Breaks for Pensions is not yet possible, and estimates that are only available for a few countries are not included in the overview calculations in this report, but only presented as a memorandum item (see below).

\section{II.4.3.2. Which tax breaks are included in the calculations and which are not?}

125. In many OECD countries (e.g., Germany and France) support for families with children is embedded in the tax unit. Although these measures may not establish a deviation from the national standard tax system (and thus do not establish a tax expenditure in the strict sense), such support clearly establishes financial and social support and should thus be included in the reported TBSPs. However, support for married couples is not considered as social in all OECD countries, and fiscal measures in this regard are not considered as a TBSP. The appropriate analogy is that the presence of dependent children leads to eligibility to cash benefits in social protection systems, whereas a marriage contract does not. Hence, tax advantages for married people, as exist in for example, Belgium, France, Germany and Japan, are not considered to serve a 'social purpose', and are therefore not included in the calculations (regardless of whether or not such measures are part of the basic tax structure). For example, value of support to children in France through the 'quotient familial' was reported to be around EUR 11.5 billion in 2007. ${ }^{19}$

126. Tax breaks for social purposes also encompass measures aimed at stimulating private pension take-up, e.g., tax exemptions for contributions to private pensions. However, such tax breaks on occupational and individual pension programmes are difficult to deal with, because such programmes are aimed at yielding benefits in the future and taxation and tax reliefs can be given at various stages of pension saving. Tax treatment of funded pension plans needs to be considered in three different areas:

18 As most countries currently publish information on tax expenditures on a cash basis, that convention has been followed here. However, in line with recent changes to reporting to the Revenue Statistics it is expected that estimates on the value of TBSPs on an accrual basis will become available on a crossnational basis.

The French system of income taxation considers the household as the tax unit: favourable tax treatment of families is thus an integral part of the tax system. In this system a 'quotient familial' is applied to taxable household income, which allows incomes to be taxed at a lower rate on a progressive marginal rate schedule. The 'quotient familial' is obtained by dividing total taxable household income ' $\mathrm{R}$ ' by a factor ' $\mathrm{N}$ ' which is determined by household composition. This factor $\mathrm{N}$ is the sum of the different 'household parts': spouses count as one part each, while the first two children count as half a 'household part', from the third child onwards each child counts as one 'household part' (slightly different rules benefit sole parent families and families with handicapped dependants). For example, for a couple-family with two children it is 3 , and for a couple-family with 3 children it is 4 . Obviously, at a given income level the larger the family, the lower is the quotient familial $(\mathrm{R} / \mathrm{N})$. 
- Contributions to programmes could be by employers or employees, out of taxed or untaxed income.

- The funds which invest pension contributions on behalf of those contributing could be taxed or untaxed.

- $\quad$ The payment of pension or annuity or lump sum benefits at the end of the contributions period could be taxed or untaxed.

Due to the complexity of calculations arising from these issues and the different methods that can be used across countries, there is no comparable data set available on the value of tax breaks for pensions. There is some data available on the cost to public budgets -- on a cash basis -- of the current tax system in the current financial year. These data abstract from the effects the current tax system may have on revenues in future years.

\section{II.4.4. The net social expenditure framework: a concise overview}

127. A cross-country comparison of social expenditure indicators requires that information on gross spending and the role of the tax system in the pursuit of social policy is integrated in a framework that derives net social expenditure indicators. Table II.6 presents a schematic overview of this framework (below, the numbers/letters in between brackets refer to the appropriate line in this Table).

- $\quad$ First of all, direct taxes clawed-back by the Exchequer and the imputed value of indirect taxation on goods consumed out of public benefits are subtracted from gross public social expenditure (1) to obtain Net direct public social expenditure (2). Subsequently, as the value of tax breaks for social purposes (excluding pensions) that are similar to cash benefits (T1) is used for consumption, the imputed value of the indirect taxation on these items is subtracted to obtain Net TBSPs similar to cash benefits (4). The value of TBSPs towards current private benefits (T2) is added to obtain net current public social expenditure (6). From the government perspective, net public social spending gives a better impression of budgetary efforts in the social field and the proportion of net social output reallocated to benefit recipients.

- In order to measure the social support that is provided under government control, mandatory private benefits should also be included, and account taken of the fact that these benefits are also subject to direct and indirect taxation. Net government-controlled social expenditure is captured under the heading of net publicly mandated social expenditure (9). Finally, the gross voluntary private benefits are also adjusted for direct and indirect taxation: net direct voluntary private social expenditure (11).

- $\quad$ Adding together these net public, mandatory private and voluntary benefits gives an indicator on net total social expenditure (13), which quantifies the proportion of an economy's domestic production at the disposal of recipients of social benefits. However, as noted above, the tax breaks towards current private social benefits (T3), are tantamount to financing private social benefits. Thus, while these TBSPs are clearly a public expenditure item, they finance private benefits and simply adding net public social expenditure to net private social expenditure would overestimate the amount of support received by households. Therefore, net total social expenditure (13) is the sum of net current public social expenditure (6) and net direct private social expenditure (12) minus TBSPs towards 
current private social benefits (T2) ${ }^{20}$ Net total social expenditure identifies that proportion of an economy's domestic production to which recipients of social benefits lay claim.

128. Finally, the net social spending indicators are related to GDP at factor cost rather than GDP at market prices - the most frequently used indicator on the size of an economy. The reason for this is that, since adjustment has been made to benefits for the value of indirect taxation, the denominator (GDP) has to be adjusted similarly. As GDP at factor cost does not include the value of indirect taxation and government subsidies to private enterprises and public corporations, it seems the most appropriate indicator for international comparisons. Nevertheless, to facilitate comparisons with gross spending to GDP quota, Annex I.2 includes the net spending indicators to GDP at market prices. This annex also relates net spending indicators to national (rather than domestic) income.

Table II.6: From gross to net social expenditure: a concise overview

\begin{tabular}{|c|c|c|}
\hline$+/-$ & Line \# & Item \\
\hline & 1. & Gross direct public social expenditure \\
\hline \multirow[t]{2}{*}{-} & & Direct taxes and social contributions paid out of public cash benefits \\
\hline & 2. & Net cash direct public social expenditure \\
\hline \multirow[t]{2}{*}{-} & & Indirect taxes on private consumption financed by net cash transfers \\
\hline & 3. & Net direct public social expenditure \\
\hline+ & T1 & Tax breaks for social purposes that mirror cash benefits \\
\hline \multirow[t]{2}{*}{-} & & Indirect taxes on private consumption financed by tax breaks similar to cash benefits \\
\hline & 4 & Net TBSPs similar to cash benefits \\
\hline \multirow[t]{4}{*}{+} & T2 & Tax breaks for social purposes towards current private social benefits \\
\hline & 5 & Net TBSPs (not including pensions) \\
\hline & 6. & Net current public social expenditure [3+5] \\
\hline & 7. & Gross mandatory private social expenditure \\
\hline \multirow{5}{*}{ - } & & Direct taxes and social contributions paid out of mandatory private cash benefits \\
\hline & & Indirect taxes on consumption purchased out of net mandatory private cash \\
\hline & 8. & Net direct mandatory private social expenditure \\
\hline & 9. & Net publicly mandated social expenditure $[6+8]$ \\
\hline & 10. & Gross voluntary private social expenditure \\
\hline \multirow{5}{*}{ - } & & Direct taxes and social contributions paid out of voluntary private cash benefits \\
\hline & & Indirect taxes on consumption purchased out of net voluntary private cash benefits \\
\hline & 11. & Net direct voluntary private social expenditure \\
\hline & 12. & Net direct private social expenditure $[8+11]$ \\
\hline & 13. ${ }^{1}$ & Net total social expenditure $[6+12-\mathrm{T} 2]$ \\
\hline
\end{tabular}

Tax adjustments in the shaded areas.

1. In order to avoid double counting, net total social expenditure is obtained by adding up net public and net private social expenditure while subtracting tax breaks towards current private benefits.

20 Ideally, the value of tax breaks aimed at stimulating private benefit provision would be netted out against the direct and indirect taxes levied on the private benefits it generated. However, as noted above, it is not possible to determine to what extent these TBSPs actually affect take-up of private benefits, and therefore this calculation was not attempted. 


\section{REFERENCES}

ADB (2006), Social Protection Index - for Committed Poverty Reduction, Asian Development Bank, Manila, the Philippines (www.adb.org/Documents/Books/Social-Protection/default.asp).

ADB (2008), Social Protection Index - for Committed Poverty Reduction-Volume 2: Asia, Asian Development Bank, Manila, the Philippines (www.adb.org/Documents/Books/SocialProtection/Volume2).

Adema, W. and M. Ladaique (2009) 'How Expensive is the Welfare State? Gross and Net Indicators in the OECD Social Expenditure Database (SOCX)', OECD Social, Employment and Migration Working Papers no. 92, OECD, Paris (www.oecd.org/els/workingpapers).

Adema, W. M. Einerhand, B. Eklind, J. Lotz and M. Pearson, (1996), "Net Public Social Expenditure", Labour Market and Social Policy Occasional Papers, No. 19, OECD, Paris (www.oecd.org/els/workingpapers).

Adema, W. and P. Whiteford (2010), 'Public and Private Social Expenditure', in: The Oxford Handbook of the Welfare State, Oxford University Press.

Australian Government (2009), Tax Expenditure Statement 2007, The Treasury, Canberra.

Caminada, C.L.J. and K.P. Goudswaard (2005), “Are Public and Private Social Expenditures Complementary?”, International Advances in Economic Research Vol. 11(2), pp. 175-189.

Castles, F. (2004), The Future of the Welfare State, Oxford University Press.

Castles, F. (2008), What Welfare States Do: A Disaggregated Expenditure Approach, Journal of Social Policy, Vol. 38(1), pp. 45-62, Cambridge University Press.

Castles, F.G. and H. Obinger (2007), "Social Expenditure and the Politics of Redistribution" Journal of European Social Policy, August 2007, Vol. 17, pp. 206-222.

Darby, J. and J. Melitz (2007), Labour Market Adjustment, Social Spending and the Automatic Stabilizers in the OECD.

Department of Finance Canada (2008), Tax Expenditures and Evaluations, Ottawa.

European Commission (2009), Ageing Report: Economic and budgetary projections for the EU-27

Member States, 2008-2060

(http://ec.europa.eu/economy_finance/publications/publication14992_en.pdf). 
EUROSTAT (2008), ESSPROS Manual - The European System of integrated Social PROtection Statistics (ESSPROS), Luxembourg

(http://epp.eurostat.ec.europa.eu/portal/page/portal/social_protection/publications).

EUROSTAT (2010), European Social Statistics, Social Protection, Expenditure and Receipts, Data 19992007, Luxembourg.

Fishback, P.V. (2010), Social Welfare Expenditures in the United States and the Nordic Countries: 19002003, NBER Working Paper No. 15982 www.nber.org/papers/w15982.

Gho, K-H., Y-S. Chang and J-W Kang (2010), Estimation of Net Social Expenditure in Korea on the Basis of the OECD Guidelines: 1990-2007, OECD/Korea Policy Centre - Health and Social Policy Programme SOCX Technical Paper, Korea.

Government of Ireland (2008), Office of the Revenue Commissioners, Statistical Report 2008, Dublin.

Grubb, D. and A. Puymoyen (2008), "Long Time-Series for Public Expenditure on Labour Market Programmes", Social, Employment and Migration Working Papers, No. 73, OECD, Paris.

ILO (2005), ILO Social Security Inquiry, First Inquiry 2005, Manual, International Labour Office, Geneva (www.ilo.org/public/english/protection/secsoc/downloads/stat/ssimane.pdf).

INPDAP (2008), Trattamenti di Fine Servizio e di Fine Rapporto dello Stato e degli Enti Locali, Analisi statistico - finanziaria, Anno 2007.

Kirkegaard, J.K. (2009), 'Did Reagan Rule In Vain? A Closer Look at True Expenditure Levels in the United States and Europe', Policy Brief 09-1 from Peterson Institute for International Economics http://www.iie.com/publications/interstitial.cfm?ResearchID=1096.

Koning, M.A., R. Saitua Nistal and J. Ebregt (2006), "Woningmarkteffecten van aanpassing fiscale behandeling eigen woning", CPB Document, No. 128, Centraal Planbureau, the Netherlands.

Ministère de l'Écologie, du Développement et de l'Aménagement durables de la France (2007),

Compte du logement 2005 et premiers résultats 2006

(www.statistiques.equipement.gouv.fr/IMG/pdf/RAPPORT_leger_cle03512b.pdf).

Ministry of Labor (2009), Retirement Pension Benefits statistics, Seoul, Korea.

OECD (1996), "Social Expenditure Statistics of OECD Member Countries - provisional version", Labour Market and Social Policy Occasional Papers, No. 17, OECD, Paris.

OECD (1999), Social Expenditure Database 1980-1996, OECD, Paris.

OECD (2000a), Social Expenditure Database 1980-1997, OECD, Paris.

OECD (2000b), A System of Health Accounts, Version 1.0, OECD, Paris.

OECD (2001), Social Expenditure Database 1980-1998 - $3^{\text {rd }}$ edition, OECD, Paris.

OECD (2004), Social Expenditure Database 1980-2001.

OECD (2007), Social Expenditure Database 1980-2003. 
OECD (2008), Growing Unequal? Income Distribution and Poverty in OECD Countries, OECD, Paris (www.oecd.org/els/social/inequality).

OECD (2009), Social Expenditure Database 1980-2005 (www.oecd.org/els/social/expenditure).

OECD (2010a), OECD Revenue Statistics, 1965-2009, OECD, Paris. (www.oecd.org/ctp/revenuestats)

OECD (2010b), Education at a Glance, OECD, Paris.

OECD (2010c), Insurance Statistics Yearbook 1999-2008: 2010 Edition, OECD, Paris.

OECD (2010d), OECD Health Data, OECD, Paris. (www.oecd.org/health/healthdata)

OECD (2010e), National Accounts of OECD Countries: Detailed tables, Volume II, OECD, Paris.

OECD (2011a), Economic Crisis and Beyond: Social Policies for the Recovery, Documentation for the 2011 OECD Ministerial Meeting on Social Policy. (www.oecd.org/social/ministerial)

OECD (2011b), Employment Outlook, OECD, Paris. (www.oecd.org/employment/outlook)

OECD (2011c), Pensions at a Glance, OECD, Paris. (www.oecd.org/els/social/pensions/PAG)

OECD (2011d), OECD Taxing Wages, OECD, Paris. (www.oecd.org/ctp/taxingwages)

OECD Social Expenditure database (SOCX, www.oecd.org/els/social/expenditure)

OECD Family database (www.oecd.org/els/social/family/database)

OECD Labour Force Statistics database (www.oecd.org/employment)

OECD Tax-Benefit models database (www.oecd.org/els/social/workincentives)

OECD Education database (www.oecd.org/education/database)

OMB (2009), Analytical Perspectives, Budget of the United States Government, Fiscal year 2009, Office of Management and Budget, Washington DC.

Oxley, H., et al. (1990), "The Public Sector- issues for the 1990s", Economics Department Working Papers, No. 90 (www.oecd.org/eco/working_papers).

Pearson, M. and J. Martin (2005), "Should We Extend the Role of Private Social Expenditure?", Social, Employment and Migration Working Papers, No. 23, OECD, Paris.

Ramesh, M. (2005), "Social Security through Compulsory Savings: The Case of Singapore", in: A Glance at Social Policy Issues in Asia - Proceedings of the International Symposium for the opening of the Joint OECD/Korea Regional Centre on Health and Social Policy, Seoul.

SNA (1993), System of National Accounts 1993, CEC-EUROSTAT, IMF, OECD, UN and the World Bank, Brussels/Luxembourg, New York, Paris, Washington, DC. 
Townsend, P. (2007), "The Right to Social Security and National Development: Lessons from OECD Experience for Low-Income Countries", Issues in Social Protection Discussion Paper No. 18 (Available at SSRN: http://ssrn.com/abstract=958252).

Varley, R. (1986), “The Government Household Transfer database 1960-1984”, Economics Department Working Papers, No.36 (www.oecd.org/eco/working_papers).

Whiteford, P. and W. Adema (2007), "What Works Best in Reducing Child Poverty: A Benefit or Work Strategy?", Social, Employment and Migration Working Papers, No. 51 OECD, Paris (www.oecd.org/els/workingpapers). 


\section{ANNEX II.1. DETAILED INFORMATION ON SOURCES OF TEH OECD SOCIAL EXPENDITURE DATABASE}

\section{AII.1.1. From ESSPROS to SOCX}

For 24 European countries (EU-21, Iceland, Norway and Switzerland), data on social expenditure is provided by EUROSTAT as based on the information in their ESSPROS database (EUROSTAT, 2010). The definitions of social expenditure that are used by SOCX and ESSPROS are similar, but there are differences in coverage and categorization. Table A.II.1.1a presents an overview.

Table A.II.1.1a From ESSPROS to SOCX: a brief overview

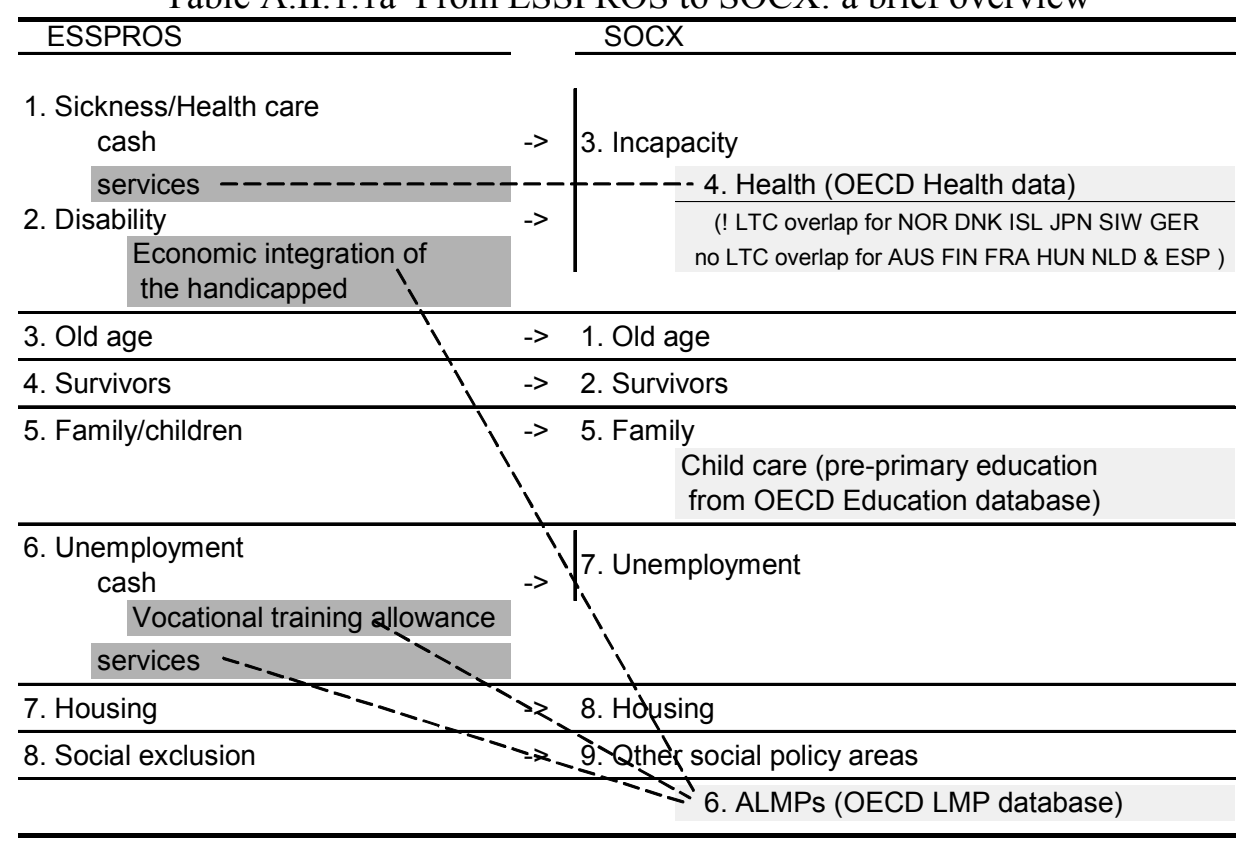

Note: ESSPROS can also be downloaded from the EUROSTAT website via http://epp.eurostat.ec.europa.eu

Then click: $>$ Statistics $>>$ Social protection

All ESSPROS social protection benefits are included in SOCX, except those in:

- Sickness /Health care services, which are taken from OECD Health data

- Programmes for the economic integration of the handicapped, and vocational training allowance for the unemployed and unemployment services, which are taken from the OECD Labour Market Programmes database (Grubb and Puymoyen, 2008) 
To regroup ESSPROS items into SOCX, the following adjustments have to be made:

(1) ESSPROS Social protection benefits

- (2) ESSPROS Economic integration of the disabled

- (3) ESSPROS Sickness benefits in kind

- (4) ESSPROS Vocational training allowance and unemployment benefits in kind

$+(5)$ Health services (OECD Health data)

+ (6) Child-care - pre-primary school (OECD Education database, for some countries)

+ (7) Active labour market programmes (OECD ALMP database)

(11) SOCX Public social expenditure

+ (8) SOCX Mandatory private social expenditure including mandatory private ESSPROS schemes

+ (9) SOCX Voluntary private social expenditure including:

voluntary private ESSPROS schemes, and

(10) Health private insurance (OECD Health data)

Example for Sweden for 2007:

Table A.II.1.1b Passage from Esspros to SOCX (public / mandatory-voluntary private) Passage from Esspros to SOCX (public / mandatory-voluntary private) SWEDEN, in millions of Swedish Kronas

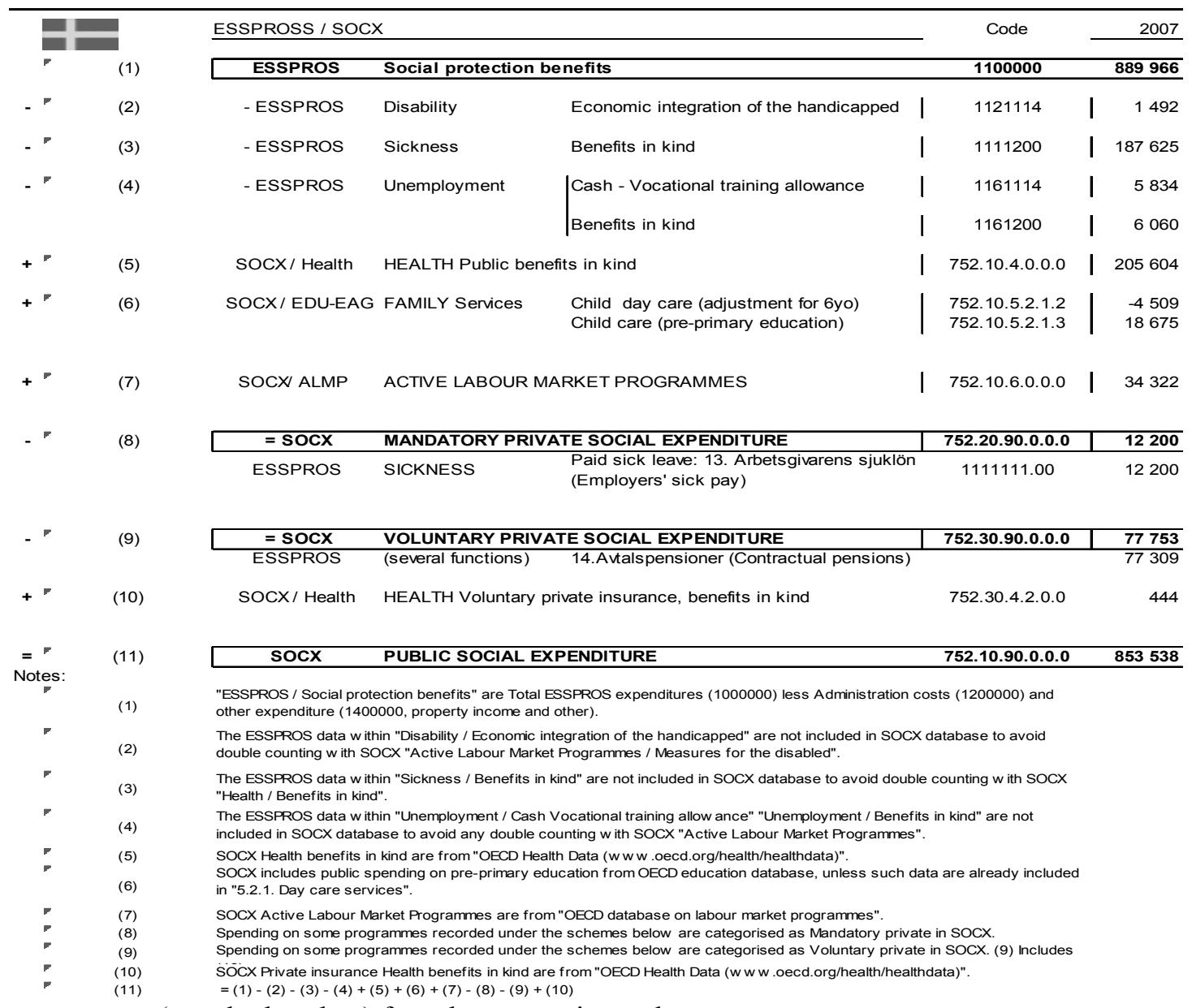

See country notes (attached to data) for other countries and years. 


\section{AII.1.2. OECD Health data}

Data in SOCX on public expenditure on health are not taken from the SOCX-questionnaire (nor from ESSPROS for EU-countries), but for reasons of comprehensiveness are taken from OECD (2010a) Health Data 2010. However, including these data in SOCX raises the possibility of introducing inconsistencies vis-à-vis health-related spending items recorded elsewhere in SOCX. For some countries there is an issue with items recorded as spending on services for elderly and/or the disabled provided by institutions other than hospitals also being included under public expenditure on health.

From countries for which information is currently available, estimates suggest that for 10 countries there exist overlap of spending data recorded as services for elderly and/or the disabled and public expenditure on health. For Denmark, Iceland and Norway relevant spending exceeds 1 percentage point of GDP in value.

Table A.II.1.2.a shows the overlap figures, and Table A.II.1.2.b shows total public spending on health (including long-term-care overlap).

Table A.II.1.2.a Estimation of overlap between OECD Health spending and SOCX

In national currency, millions

\begin{tabular}{|c|c|c|c|c|c|c|c|c|c|c|c|c|c|c|}
\hline & 1980 & 1981 & 1982 & 1983 & 1984 & 1985 & 1986 & 1987 & 1988 & 1989 & 1990 & 1991 & 1992 & 1993 \\
\hline Austria & $\mathrm{m}$ & $\mathrm{m}$ & $\mathrm{m}$ & $\mathrm{m}$ & $\mathrm{m}$ & $\mathrm{m}$ & $\mathrm{m}$ & $\mathrm{m}$ & $\mathrm{m}$ & $m$ & 1046 & 1080 & 1134 & 1415 \\
\hline Denmark & 9079 & 10345 & 11928 & 12728 & 13128 & 14283 & 14907 & 15710 & 16576 & 17197 & 18731 & 19126 & 19433 & 19620 \\
\hline Finland & $\mathrm{x}$ & $\mathrm{x}$ & $\mathrm{x}$ & $\mathrm{x}$ & 105 & 121 & 117 & 119 & 122 & 125 & 151 & 162 & 156 & 159 \\
\hline France & $\mathrm{m}$ & $\mathrm{m}$ & $\mathrm{m}$ & $\mathrm{m}$ & $\mathrm{m}$ & $\mathrm{m}$ & $\mathrm{m}$ & $\mathrm{m}$ & $\mathrm{m}$ & $\mathrm{m}$ & 2260 & 2500 & 2791 & 3103 \\
\hline Germany & $\mathrm{a}$ & $\mathrm{a}$ & $\mathrm{a}$ & $\mathrm{a}$ & $\mathrm{a}$ & $\mathrm{a}$ & $\mathrm{a}$ & $\mathrm{a}$ & $\mathrm{a}$ & $\mathrm{a}$ & $\mathrm{a}$ & $\mathrm{a}$ & $\mathrm{a}$ & $\mathrm{a}$ \\
\hline Iceland & $\mathrm{m}$ & $\mathrm{m}$ & $\mathrm{m}$ & $\mathrm{m}$ & $\mathrm{m}$ & $\mathrm{m}$ & $\mathrm{m}$ & $\mathrm{m}$ & $\mathrm{m}$ & $\mathrm{m}$ & 3456 & 3831 & 3946 & 4189 \\
\hline Japan & $\mathrm{m}$ & $\mathrm{m}$ & $\mathrm{m}$ & $\mathrm{m}$ & $\mathrm{m}$ & $\mathrm{m}$ & $\mathrm{m}$ & $\mathrm{m}$ & $\mathrm{m}$ & $\mathrm{m}$ & $\mathrm{m}$ & $\mathrm{m}$ & $\mathrm{m}$ & $\mathrm{m}$ \\
\hline Norway & 3276 & $\mathrm{~m}$ & $\mathrm{~m}$ & $\mathrm{~m}$ & $\mathrm{~m}$ & 6373 & $\mathrm{~m}$ & $\mathrm{~m}$ & 18631 & 19928 & 14867 & 16550 & 18335 & 19030 \\
\hline Sweden & $\mathrm{m}$ & $\mathrm{m}$ & $\mathrm{m}$ & $\mathrm{m}$ & $\mathrm{m}$ & $\mathrm{m}$ & $\mathrm{m}$ & $\mathrm{m}$ & $\mathrm{m}$ & $\mathrm{m}$ & $\mathrm{m}$ & $\mathrm{m}$ & $\mathrm{m}$ & 10525 \\
\hline \multirow[t]{2}{*}{ Switzerland } & $\mathrm{m}$ & $\mathrm{m}$ & $\mathrm{m}$ & $\mathrm{m}$ & $\mathrm{m}$ & $\mathrm{m}$ & $\mathrm{m}$ & $\mathrm{m}$ & $\mathrm{m}$ & $\mathrm{m}$ & 1247 & 1383 & 1670 & 1808 \\
\hline & 1994 & 1995 & 1996 & 1997 & 1998 & 1999 & 2000 & 2001 & 2002 & 2003 & 2004 & 2005 & 2006 & 2007 \\
\hline Austria & 1857 & 1918 & 1889 & 1923 & 2031 & 2155 & 2196 & 2223 & 2257 & 2320 & 2435 & 2539 & 2694 & 2829 \\
\hline Denmark & 20672 & 21822 & 23181 & 24138 & 21395 & 21641 & 22274 & 23357 & 24784 & 25787 & 27240 & 28203 & 30047 & 29049 \\
\hline Finland & 150 & 135 & 131 & 133 & 128 & 127 & 133 & 141 & 142 & 147 & 153 & 164 & 166 & 177 \\
\hline France & 3408 & 10078 & 10799 & 11050 & 11368 & 11700 & 12356 & 12974 & 14702 & 15598 & 16903 & 18354 & 20015 & 21759 \\
\hline Germany & $\mathrm{a}$ & 1268 & 2195 & 2326 & 2555 & 2824 & 2951 & 2991 & 3116 & 3135 & 3189 & 3314 & 3385 & 3484 \\
\hline Iceland & 4227 & 4661 & 5155 & 5521 & 6351 & 7263 & 8238 & 9130 & 12116 & 15933 & 17443 & 19277 & 21556 & 22937 \\
\hline Japan & $\mathrm{m}$ & 22599 & 25044 & 27708 & 30383 & 32854 & 1669923 & 1746995 & 1989048 & 2065600 & 2140810 & 2065927 & 1860444 & 1933144 \\
\hline Norway & 20464 & 21659 & 22610 & 23644 & 25785 & 27680 & 29649 & 32203 & 30787 & 32389 & 34045 & 35304 & 37980 & 41052 \\
\hline Sweden & 10768 & 11866 & 12871 & 13248 & 14105 & 14835 & 15702 & 16819 & 18148 & 19170 & 19454 & 20141 & 21045 & 22360 \\
\hline Switzerland & 1908 & 1996 & 2076 & 2181 & 2306 & 2351 & 2450 & 2652 & 2793 & 3019 & 3146 & 3264 & 3348 & 3460 \\
\hline
\end{tabular}


Table A.II.1.2.b Total public spending on Health

\begin{tabular}{|c|c|c|c|c|c|c|c|c|c|c|c|c|c|c|}
\hline & 1980 & 1981 & 1982 & 1983 & 1984 & 1985 & 1986 & 1987 & 1988 & 1989 & 1990 & 1991 & 1992 & 1993 \\
\hline Australia & 5869 & 6545 & 7076 & 8498 & 10555 & 11863 & 13235 & 14523 & 15896 & 17491 & 18734 & 19799 & 20926 & 21968 \\
\hline \multirow{2}{*}{\multicolumn{15}{|c|}{$\begin{array}{l}\text { Canada } \\
\text { Chile }\end{array}$}} \\
\hline & & & & & & & & & & & & & & \\
\hline Czech Republic & 15180 & & & & & 19960 & & & & 26310 & 30452 & 38242 & 43597 & 69289 \\
\hline Denmark & 30861 & 34543 & 39377 & 41651 & 43438 & 47112 & 48220 & 52459 & 55617 & 57257 & 58030 & 60031 & 62461 & 65193 \\
\hline \multicolumn{15}{|l|}{ Estonia } \\
\hline Finland & 1654 & 1937 & 2231 & 2504 & 2789 & 3198 & 3512 & 3878 & 4264 & 4831 & 5592 & 6099 & 5940 & 5258 \\
\hline France & 25034 & & & & & 46755 & & & & & 66173 & 70333 & 75216 & 79563 \\
\hline Germany & 52266 & 56535 & 57731 & 59390 & 62950 & 66854 & 69822 & 72427 & 77592 & 76089 & 82521 & & 128415 & 131002 \\
\hline Greece & 224 & & & & & & & 994 & 980 & 1323 & 1554 & 1864 & 2408 & 3027 \\
\hline Hungary & & & & & & & & & & & & 160600 & 200500 & 241000 \\
\hline Iceland & 884 & 1434 & 2355 & 4457 & 5329 & 7631 & 10732 & 14418 & 18927 & 22635 & 25105 & 27970 & 27783 & 28513 \\
\hline Ireland & 881 & 1026 & 1157 & 1255 & 1319 & 1410 & 1461 & 1455 & 1458 & 1551 & 1599 & 1801 & 2018 & 2209 \\
\hline \multicolumn{15}{|l|}{ Israel } \\
\hline Italy & & & & & & & & & 33082 & 35884 & 42975 & 48252 & 49634 & 49363 \\
\hline Japan & 11162700 & 12082600 & 12996950 & 14075300 & 14531300 & 15295300 & 16273550 & 17237860 & 18067320 & 19043900 & 20261900 & 21839400 & 23304900 & 24836300 \\
\hline Korea & 314964 & 405045 & 527637 & 683505 & 830166 & 969444 & 1032827 & 1200012 & 1607190 & 2120751 & 2937842 & 3200742 & 3788613 & 4259813 \\
\hline Luxembourg & 209 & 232 & 253 & 262 & 284 & 308 & 331 & 393 & 419 & 466 & & 554 & 614 & \\
\hline Mexico & $20 \%$ & 252 & 200 & 202 & 204 & 500 & 301 & 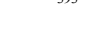 & 419 & 400 & 14424 & 21645 & 27077 & 31431 \\
\hline Netherlands & 8394 & 9037 & 9742 & 9994 & 10127 & 10393 & 10466 & 10807 & 11058 & 12224 & 13094 & 14501 & 16313 & 17223 \\
\hline New Zealand & 1216 & 1580 & 1695 & 1852 & 1953 & 2072 & 2519 & 3169 & 3681 & 3997 & 4183 & 4436 & 4475 & 4488 \\
\hline Norway & 18628 & 21204 & 23798 & 26871 & 28711 & 31092 & 34988 & 40543 & 42920 & 44223 & 46564 & 52392 & 54697 & 56318 \\
\hline Poland & & & & & & & & & & & 2710 & 4019 & 5821 & 7308 \\
\hline Portugal & 267 & 340 & 364 & 398 & 483 & 690 & 922 & 1026 & 1325 & 1421 & 2059 & 2483 & 2734 & 3180 \\
\hline \multicolumn{15}{|l|}{$\begin{array}{l}\text { Slovak Republic } \\
\text { Swaun }\end{array}$} \\
\hline Slovenia & & & & & & & & & & & & & & \\
\hline Spain & 4126 & 4688 & 5570 & 6900 & 7249 & 7803 & 8720 & 9872 & 12121 & 13818 & 16413 & 18258 & 20620 & 22069 \\
\hline Sweden & 46068 & 50762 & 56094 & 62136 & 68140 & 70458 & 74629 & 80901 & 86848 & 96821 & 107189 & 110492 & 111169 & 115860 \\
\hline Switze rland & & & & & & 9538 & 10203 & 10869 & 11719 & 13102 & 14201 & 16179 & 17603 & 18331 \\
\hline Turkey & 0 & & & & 0 & & 1 & 1 & 2 & 4 & 9 & 15 & 28 & 49 \\
\hline United Kingdom & 11633 & 13374 & 14081 & 15928 & 16781 & 17889 & 19164 & 21190 & 23202 & 25424 & 28039 & 31511 & 35730 & 37955 \\
\hline United States & 101227 & 117198 & 130032 & 143582 & 155996 & 169575 & 185604 & 203162 & 220015 & 245150 & 275064 & 312773 & 349793 & 384977 \\
\hline
\end{tabular}

\begin{tabular}{|c|c|c|c|c|c|c|c|c|c|c|c|c|c|c|}
\hline & 1994 & 1995 & 1996 & 1997 & 1998 & 1999 & 2000 & 2001 & 2002 & 2003 & 2004 & 2005 & 2006 & 2007 \\
\hline Australia & 23324 & 25264 & 27033 & 29533 & 31539 & 35209 & 38049 & 40847 & 44963 & 47381 & 52733 & 56430 & 61459 & 67865 \\
\hline Austria & 12011 & 12299 & 12559 & 13636 & 14484 & 15375 & 15860 & 16313 & 16777 & 17356 & 18341 & 19294 & 20038 & 21331 \\
\hline Belgium & & 13433 & 14144 & & & & & & & 19661 & 21813 & 22411 & 22969 & 24530 \\
\hline Canada & 52023 & 52238 & 52325 & 54404 & 58371 & 61209 & 66991 & 72253 & 77172 & 83589 & 88962 & 95349 & 101576 & 108425 \\
\hline Chile & & 728447 & 854950 & 969178 & 1126016 & 1221516 & 1402631 & 1578078 & 1705958 & 1868529 & 2046691 & 2295867 & 2672608 & 3141876 \\
\hline Czech Republic & 81126 & 93310 & 102399 & 108934 & 119651 & 123453 & 129430 & 141157 & 157899 & 172159 & 180276 & 188647 & 194632 & 203515 \\
\hline Denmark & 67757 & 68364 & 72378 & 75489 & 79410 & 84508 & 88147 & 94546 & 99744 & 109342 & 116045 & 122794 & 131777 & 139225 \\
\hline Estonia & & & & & & 3803 & 3933 & 4166 & 4547 & 5245 & 5880 & 6740 & 7700 & 9805 \\
\hline Finland & 5124 & 5440 & 5736 & 5940 & 6180 & 6477 & 6789 & 7416 & 8090 & 8608 & 9146 & 9756 & 10369 & 10981 \\
\hline France & 81433 & 98711 & 101255 & 103258 & 106586 & 110276 & 115252 & 121233 & 129799 & 137939 & 144843 & 151864 & 157005 & 163651 \\
\hline Germany & 140841 & 152196 & 160157 & 158361 & 161178 & 165445 & 169292 & 175124 & 180770 & 184031 & 179829 & 184224 & 188074 & 194337 \\
\hline Greece & 3458 & 4003 & 4457 & 4855 & 5159 & 5807 & 6444 & 7832 & 8264 & 9208 & 9509 & 11212 & 12616 & 13212 \\
\hline Hungary & 317400 & 352300 & 405000 & 482500 & 551704 & 606479 & 664488 & 754414 & 912575 & 1141433 & 1205237 & 1323477 & 1395516 & 1328712 \\
\hline Iceland & 29687 & 31269 & 33138 & 34972 & 42130 & 50011 & 52645 & 58380 & 67879 & 71344 & 74638 & 78807 & 87431 & 98160 \\
\hline Ireland & 2339 & 2560 & 2734 & 3222 & 3610 & 4133 & 4841 & 6016 & 7059 & 7906 & 8741 & 9357 & 10077 & 10989 \\
\hline Israel & & 14818 & 17722 & 19387 & 20668 & 21995 & 23740 & 25543 & 26748 & 26410 & 26654 & 27622 & 28910 & 29786 \\
\hline Italy & 49073 & 48659 & 52273 & 56879 & 59332 & 62110 & 69639 & 76550 & 80455 & 83017 & 91472 & 97379 & 102571 & 102762 \\
\hline Japan & 25957100 & 28190468 & 29414100 & 29354152 & 29718844 & 30439404 & 31435615 & 32314548 & 31896184 & 32299437 & 32754617 & 33860946 & 33309886 & 34254816 \\
\hline Korea & 4848501 & 5923228 & 7521910 & 8742569 & 9859082 & 11651526 & 13148257 & 18070699 & 19012841 & 20804455 & 22783555 & 25832697 & 30251650 & 34111704 \\
\hline Luxembourg & 710 & 777 & 829 & 849 & 912 & 1040 & 1149 & 1263 & 1472 & 1751 & 2010 & 2100 & 2237 & \\
\hline Mexico & 37065 & 43697 & 53634 & 74742 & 94912 & 122868 & 142155 & 155610 & 169392 & 192961 & 231048 & 243800 & 265886 & 296166 \\
\hline Netherlands & 17611 & 18059 & 17376 & 18450 & 18744 & 19589 & 20981 & 23333 & 25773 & & & & & \\
\hline New Zealand & 4870 & 5160 & 5342 & 5750 & 6183 & 6490 & 6984 & 7455 & 8319 & 8741 & 9724 & 10775 & 11934 & 12840 \\
\hline Norway & 58381 & 62602 & 68055 & 76436 & 86715 & 95530 & 102896 & 113025 & 125239 & 133577 & 140578 & 147784 & 156465 & 170646 \\
\hline Poland & 9787 & 13460 & 18218 & 20787 & 23204 & 27153 & 28781 & 32840 & 36460 & 36797 & 39336 & 42341 & 45945 & 53601 \\
\hline Portugal & 3477 & 4137 & 4741 & 5169 & 5700 & 6362 & 7846 & 8156 & 8800 & 9851 & 10356 & 10880 & 11041 & \\
\hline Slovak Republic & & & & 1266 & 1356 & 1450 & 1532 & 1665 & 1845 & 2087 & 2402 & 2585 & 2761 & 3186 \\
\hline Slovenia & & 600 & 695 & 792 & 889 & 998 & 1136 & 1304 & 1467 & 1559 & 1657 & 1740 & 1851 & 1939 \\
\hline Spain & 22774 & 24031 & 25589 & 26781 & 28509 & 30561 & 32550 & 35079 & 37695 & 44957 & 48603 & 53145 & 58652 & 63854 \\
\hline Sweden & 117286 & 124884 & 132154 & 133149 & 140992 & 150279 & 157306 & 170654 & 184707 & 195150 & 197461 & 204504 & 214555 & 227964 \\
\hline Switzerland & 18915 & 19153 & 20411 & 20997 & 21761 & 22665 & 23748 & 25943 & 27360 & 28745 & 29784 & 30946 & 31202 & 32623 \\
\hline $\begin{array}{l}\text { Turkey } \\
\text { The }\end{array}$ & 96 & 184 & 399 & 870 & 1815 & 3047 & $\begin{array}{r}5190 \\
5190\end{array}$ & $\begin{array}{r}8438 \\
8438\end{array}$ & 13270 & 17462 & 21389 & 23987 & 30116 & 34530 \\
\hline United Kingdom & 40054 & 42003 & 44377 & 44248 & 47211 & 51817 & 54534 & 59352 & 65168 & 71055 & 78468 & 84770 & 91848 & 96713 \\
\hline United States & 419691 & 448179 & 472474 & 493826 & 508415 & 534750 & 573352 & 636137 & 692319 & 746431 & 804414 & 862802 & 937878 & 1000582 \\
\hline
\end{tabular}

Source: Wwww.oced.orgh health healthdala
OECD Heath Data 2010 - Version: June 2010

Finally, indicators on voluntary private social health expenditure are estimates on the benefits to recipients that derive from private health plans which contain an element of redistribution (such private health insurance plan are often employment-based and/or tax-advantaged). The estimates are based on OECD Health Data (see Private insurance, within Health expenditure by sources of funds). Available data on individual payments does not distinguish between individual co-payments and other out-of-pocket health expenditure. But the OECD Health Data are being refined to cover this distinction in future. By not including data on individual payments, it is thus implicitly assumed that none of the individual payments (including co-payments) are in any way subject to redistribution. This is a very strong assumption, which is unlikely to fully reflect reality, but it was judged more realistic than the alternative - to include all individual payments. The estimates on private social health benefits may thus somewhat underestimate the "true" social extent of health-care provisions. 


\section{AII.1.3. OECD Labour Market Programmes database}

Data on public spending on ALMPs (social policy area (or branch) "6" in SOCX) are taken from the OECD database on Labour Market Programmes. This database has recently been restructured to identify seven "active" categories:

1. PES and administration

2. Training

3. Job rotation and job training

4. Employment incentives

5. Supported employment and rehabilitation

6. Direct Job Creation

7. Start-Up Incentives.

To ensure consistency with the historical series as in SOCX, data prior to 1998 (for Eurostat countries) and prior to 2001 (for non-Eurostat countries) have been regrouped in the "new" classification system (see Grubb and Puymoyen, 2008, for more detail):

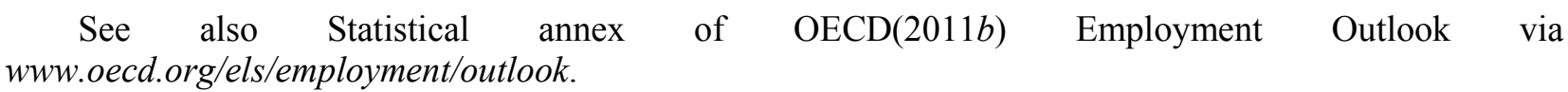

\section{AII.1.4. OECD Education database}

For reasons of comprehensiveness, SOCX collects for most countries spending figures from OECD Education database on Childcare and early education services (ISCED0) - see non-shaded background in Table A.II.1.4. All available data on public financial support for families with children participating in both formal day-care services (i.e., crèches, day-care centres and family day-care for children under 3 ) and preschool institutions (including kindergartens and day-care centres for children aged from 3 to 6) are included, from 1998 only in general, from which ISCED97 started.

To get a good comparison of childcare support, account has been taken of cross-national differences in the compulsory age of entry into primary school. For example, in some (Nordic) countries children enter primary school at age 7, while 6 year olds attend pre-primary school the year beforehand. In order to improve the comparison, expenditure on these 6 year olds was excluded (sometimes using estimates derived on basis of available data on spending on education and the number of 6 year olds). Similarly, for countries where children enter school at age 5 (and which were not already included in the childcare and pre-school data) pre-school expenditure data for Australia, New Zealand and the United Kingdom was adjusted by adding up the expenditure on 5 year olds enrolled in primary school - see adjustments in Table A.II.1.4. 


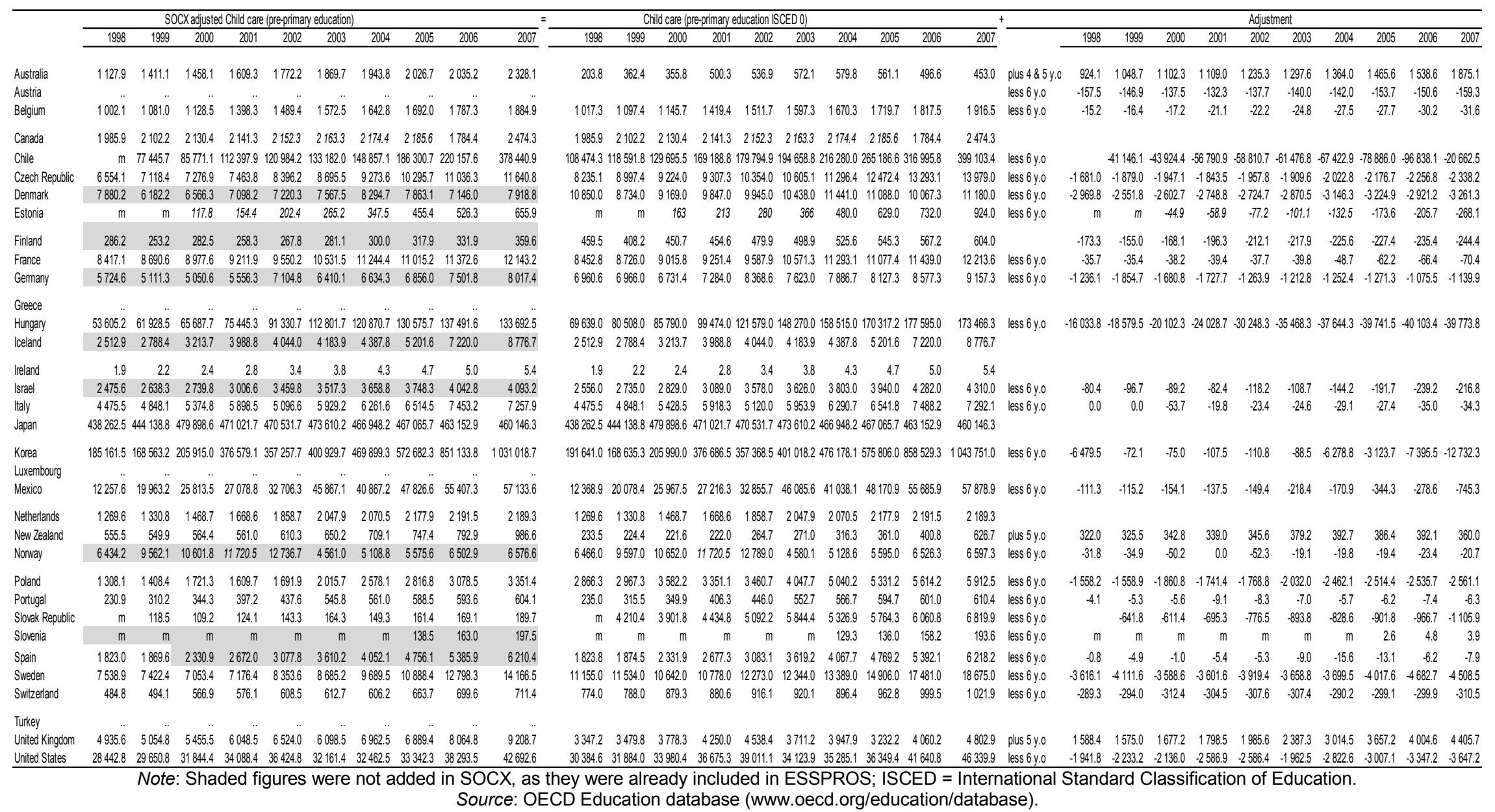




\section{ANNEX II.2. HOW TO ACCESS SOCX ELECTRONICALLY?}

As described in SOCX home webpage www.oecd.org/els/social/expenditure, SOCX data is now available using OECD.Stat available via $h t t p: / / s t a t s . o e c d . o r g$

then click on Theme $>$ Social and Welfare Statistics $>>$ Social Protection

select appropriate SOCX dataset, then click on OPEN

A OECD.Stat "user guide" can be found at top-right of OECD.Stat home page.

SOCX2007 contains three datasets:

- SOCX-AGG for main aggregates

- SOCX-REF for reference series used for calculating aggregates

- SOCX-DET for detailed expenditure at the programme level via OECD.Stat and for OECD/OLIS users

via http://dx.doi.org/10.1787/socx-data-en for other public.

See next pages for more information on each dataset.

Default views are as follows:

- years 1980, 1985, 1990, 1995, 2000, 2001, 2002, 2003, 2004, 2005, 2006 and 2007 - data for intervening years are also available by changing the Year selection at top-right of the page.

- all countries are selected for aggregated and reference series datasets.

Default selections can be modified by double-clicking on appropriate variable and selecting appropriate item(s) in the left menu.

Missing values are presented as follows:

$\mathrm{m}$ data not available;

a data do not exist;

$\mathrm{x}$ data included in another category.

! Tips:

- all variables can be moved using "drag \& drop" in headers/columns/lines as in a pivot-table;

- meta-data information are available by clicking on "i" next to specific variable/item;

- any table can be exported into Excel / Text file by clicking on appropriate icon at top-right of table

- French version of datasets is available by clicking on "version française" at top-right of screen. 


\section{AII.2.1 SOCX aggregated data are available in OECD.Stat via}

http://stats.oecd.org/wbos/default.aspx?datasetcode $=S O C X \_A G G$

Aggregated data are available by: (codes in brackets)

- source: Public (10), Mandatory private (20), both public and mandatory private (10_20)

Or Voluntary private (30)

- branch: each 9 social policy area 1 to 9 , or Total (90)

- type of expenditure: Cash benefits (1), Benefits in kind, or Total (0)

- type of programme: each of the 36 sub-areas (Table 4.1), or Total (0)

to view data by type of program "xyz", select: branch " $x$ " and type of expenditure " $y$ " - unit, as follows:

\begin{tabular}{|c|c|c|c|c|c|}
\hline & & Source & Branch & $\begin{array}{c}\text { Type of } \\
\text { expenditure }\end{array}$ & $\begin{array}{c}\text { Type of } \\
\text { programme }\end{array}$ \\
\hline at current prices in national currency, in millions & NCUR & $\mathrm{x}$ & $\mathrm{x}$ & $\mathrm{x}$ & $\mathrm{x}$ \\
\hline at constant prices (2000) in national currency, in millions & NCST & $\mathrm{x}$ & $\mathrm{x}$ & $\mathrm{x}$ & $\mathrm{x}$ \\
\hline per head, at current prices and current PPPs, in US dollars & PPPH & $\mathrm{x}$ & $\mathrm{x}$ & $\mathrm{x}$ & $\mathrm{x}$ \\
\hline per head, at constant prices (2000) and constant PPPs (2000), in US dollar: & PPPVH & $x$ & $x$ & $\mathrm{x}$ & $x$ \\
\hline in percentage of GDP & PCT_GDP & $\mathrm{x}$ & $\mathrm{x}$ & $\mathrm{x}$ & $\mathrm{x}$ \\
\hline in percentage of $\mathrm{GNI}$ & PCT_GNI & $\mathrm{x}$ & $\mathrm{x}$ & $\mathrm{x}$ & $\mathrm{x}$ \\
\hline in percentage of $\mathrm{NNI}$ & PCT_NNI & $\mathrm{x}$ & $\mathrm{x}$ & $\mathrm{x}$ & $\mathrm{x}$ \\
\hline in percentage of GOV & PCT_GOV & $x$ & $x$ & $\mathrm{x}$ & $\mathrm{x}$ \\
\hline
\end{tabular}

$\mathrm{x}$ : available.

See reference series for notes and sources.

- country: each of 34 OECD countries, or Total

- year: any year from 1980 to 2007

See below a screen capture

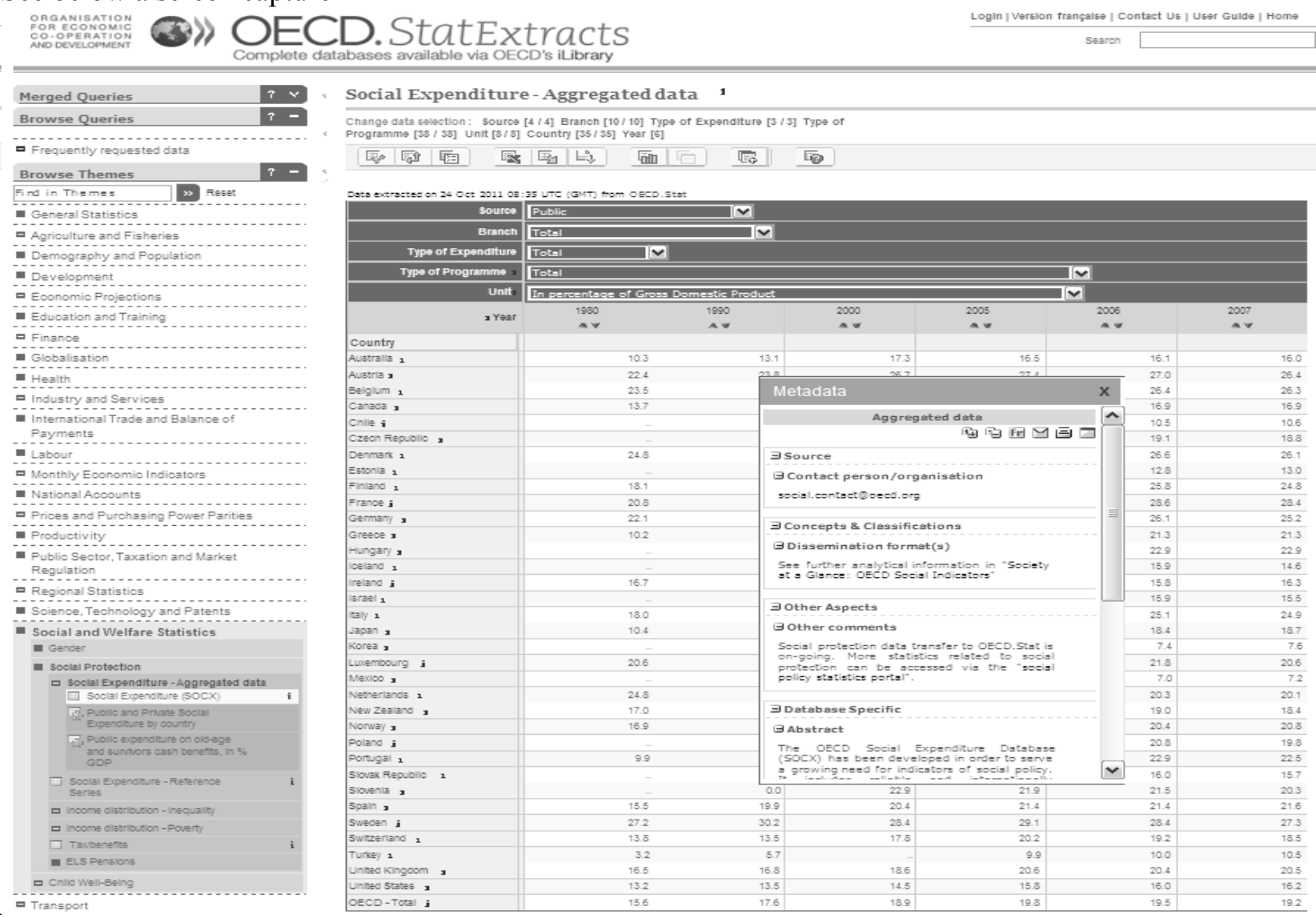




\section{AII.2.2. SOCX Reference series are available in OECD.Stat via http://stats.oecd.org/wbos/default.aspx?datasetcode $=S O C X \_R E F$}

Reference series are available as follows:

- GDP: Gross Domestic Product at current prices in national currency, in millions - GDPV: Gross Domestic Product at 2000 prices in national currency, in millions

- DEFL: Deflator for GDP, I(2000) $=100$

- GNI: Gross national income at market prices, at current prices in national currency, in millions

$=$ GDP at market prices

+ Taxes less subsidies on production and imports (net, receivable from abroad)

+ Compensation of employees (net, receivable from abroad)

+ Property income (net, receivable from abroad)

- GOV: Total general government expenditure, at current prices in national currency, in millions - PPP: Purchasing Power Parities (PPP) for GDP, National currency per US dollar

- EXC: Exchange rates, National currency per US dollar

- POP: Population, Mid-year estimates, in thousands

Source: OECD, National Accounts database.

See below a screen capture

OFy) OED.StatExtractS

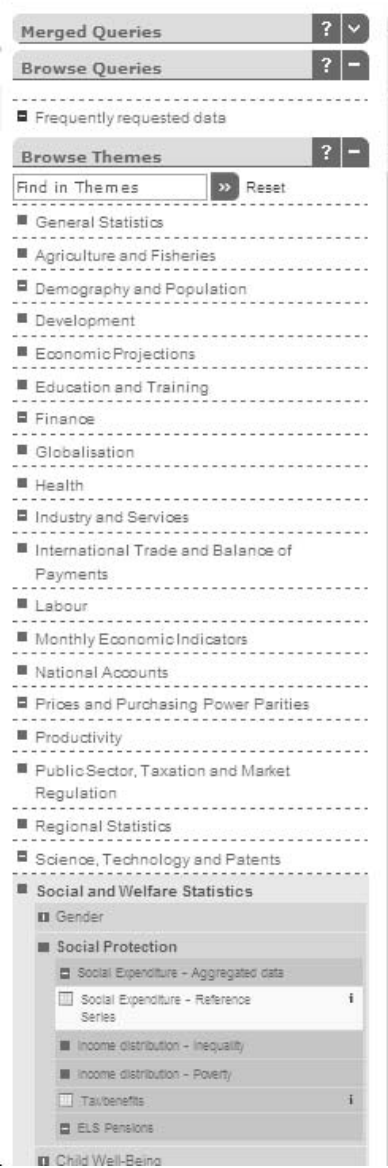

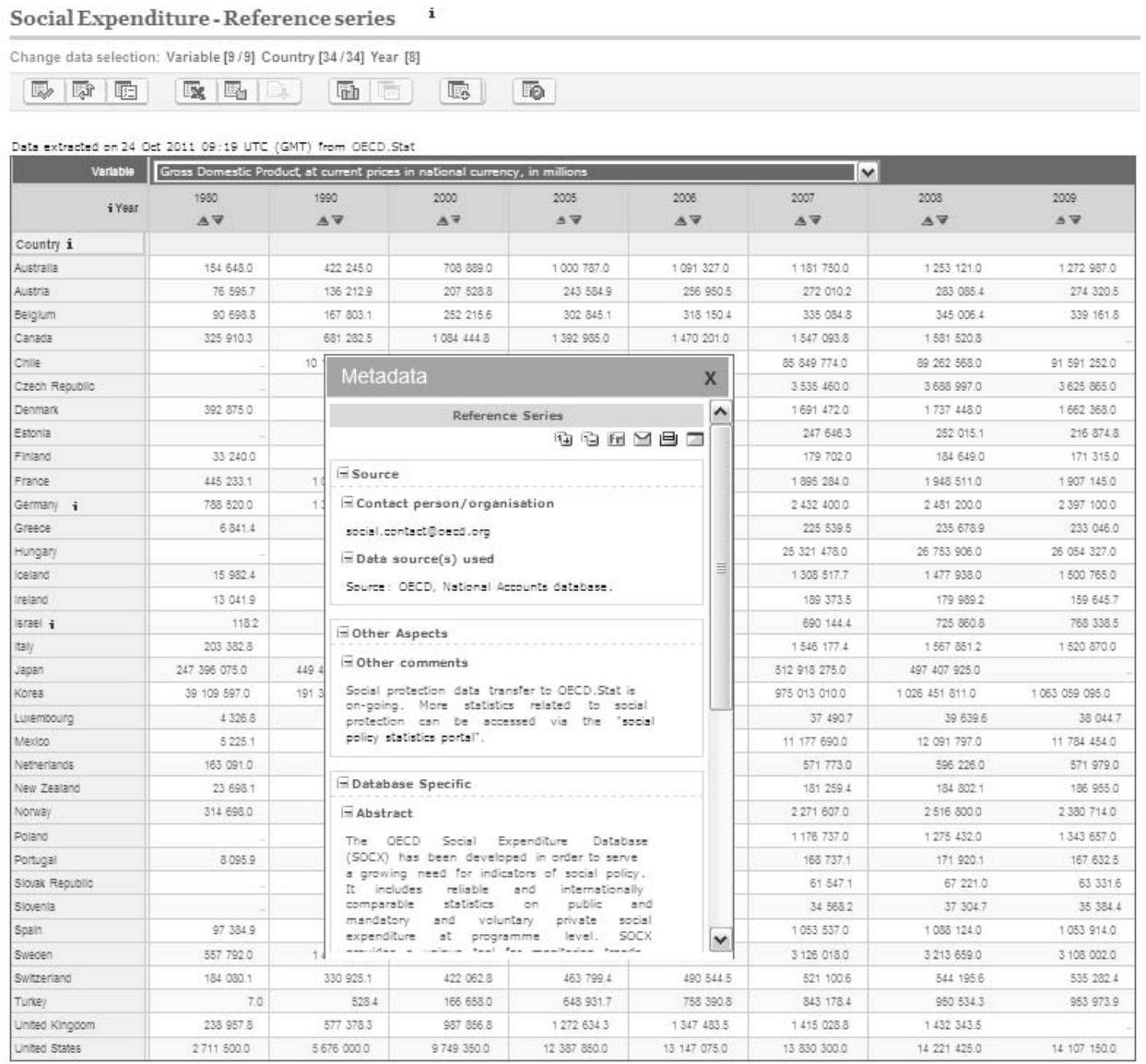




\section{A.II.2.3. SOCX Detailed expenditure at the programme level are available in OECD.Stat:}

- for OECD-OLIS users via http://dotstat.oecd.org/wbos/Index.aspx?DataSetCode=SOCX_DET

- for other public: via http://dx.doi.org/10.1787/socx-data-en

Detailed expenditures at programme level are available in OECD.stat and .IVT at:

- at current prices in national currency, in millions

(NatCur)

Detailed expenditures at programme level are also available in .XLS at:

- at constant (2000) prices in national currency, in millions

(NatCst)

- per head, at current prices and current PPPs, in US dollars

$(\mathrm{PPPH})$

- per head, at constant prices and PPPs (2000), in US dollars

$(\mathrm{PPPVH})$

- in percentage of Gross Domestic Product

(PCT_GDP)

- in percentage of Gross National Income

(PCT_GNI)

- in percentage of Net National Income

(PCT_NNI)

- in percentage of Total general government expenditure

(PCT_GOV)

Each social programme has a "unique" code, made of 6 components:

Name of programme $=$

"Country code". "Source" . "branch". "type of expenditure" . "type of programme" . "number of programme" with: (codes in brackets)

- country: ISO country code

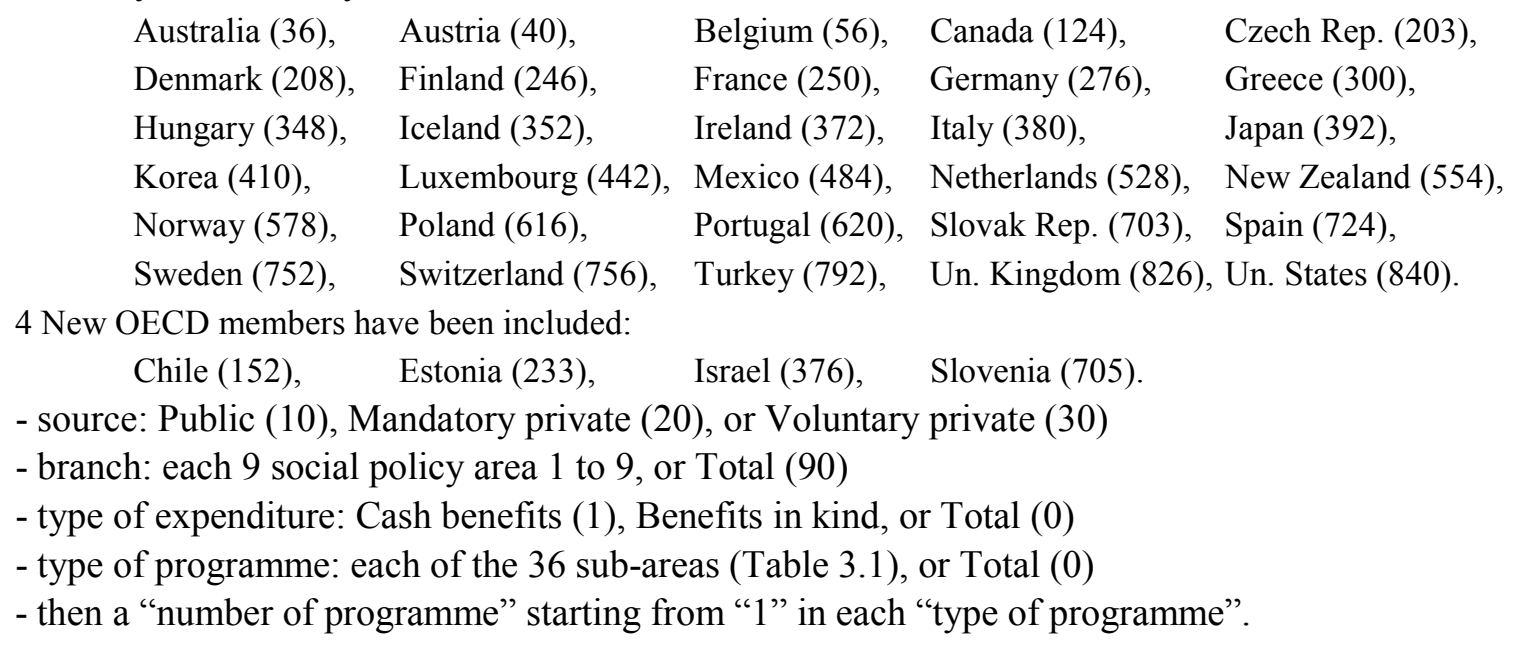

Last but not least, "country-notes" - presenting country-specific sources and definitions of social programmes - are available in both English and French as related files in OECD.Stat and in the documentation package in http://dx.doi.org/10.1787/socx-data-en 


\section{OECD SOCIAL, EMPLOYMENT AND MIGRATION WORKING PAPERS}

Most recent releases are:

No. 123 AGGREGATE EARNINGS AND MACROECONOMIC SHOCKS Andrea Bassanini (2011)

No. 122 REDISTRIBUTION POLICY AND INEQUALITY REDUCTION IN OECD COUNTRIES: WHAT HAS CHANGED IN TWO DECADES? Herwig Immervoll, Linda Richardson (2011)

No. 121 OVER-QUALIFIED OR UNDER-SKILLED Glenda Quintini (2011)

No. 120 RIGHT FOR THE JOB Glenda Quintini (2011)

No.119 THE MORAL-HAZARD AND LIQUIDITY EFFECTS OF UNEMPLOYMENT COMPENSATION IN BRAZIL: EVIDENCE AND POLICY IMPLICATIONS Andrea Bassanini (2011)

No. 118 EARLY MATERNAL EMPLOYMENT AND CHILD DEVELOPMENT IN FIVE OECD COUNTRIES Maria del Carmen Huerta, Willem Adema, Jennifer Baxter, Miles Corak, Mette Deding, Matthew C.

Gray, Wen-Jui Han, Jane Waldfogel (2011)

No. 117 WHAT DRIVES INFLOWS INTO DISABILITY?EVIDENCE FROM THREE OECD COUNTRIES Ana Llena-Nozal and Theodora Xenogiani (2011)

No. 116 COOKING, CARING AND VOLUNTEERING: UNPAID WORK AROUND THE WORLD Veerle Miranda (2011)

No. 115 THE ROLE OF SHORT-TIME WORK SCHEMES DURING THE 2008-09 RECESSION Alexander Hijzen and Danielle Venn (2010)

No. 114 INTERNATIONAL MIGRANTS IN DEVELOPED, EMERGING AND DEVELOPING COUNTRIES: AN EXTENDED PROFILE

Jean-Christophe Dumont, Gilles Spielvogel and Sarah Widmaier (2010)

No. 113 ACTIVATION POLICIES IN JAPAN

Nicola Duell, David Grubb, Shruti Singh and Peter Tergeist (2010)

No. 112 ACTIVATION POLICIES IN SWITZERLAND

Nicola Duell and Peter Tergeist with contributions from Ursula Bazant and Sylvie Cimper (2010)

No. 111 ECONOMIC DETERMINANTS AND CONSEQUENCES OF CHILD MALTREATMENT Lawrence M. Berger, Jane Waldfogel (forthcoming)

No. 110 DISTRIBUTIONAL CONSEQUENCES OF LABOR DEMAND ADJUSTMENTS TO A DOWNTURN: A MODEL-BASED APPROACH WITH APPLICATION TO GERMANY 2008-09 Herwig Immervoll, Olivier Bargain, Andreas Peichl, Sebastian Siegloch (2010)

No. 109 DECOMPOSING NOTIONAL DEFINED-CONTRIBUTION PENSIONS: EXPERIENCE OF OECD COUNTRIES'REFORMS

Edward Whitehouse (2010)

No. 108 EARNINGS OF MEN AND WOMEN WORKING IN THE PRIVATE SECTOR: ENRICHED DATA FOR PENSIONS AND TAX-BENEFIT MODELING Anna Cristina D'Addio and Herwig Immervoll (2010)

No. 107 INSTITUTIONAL DETERMINANTS OF WORKER FLOWS: A CROSS-COUNTRY/CROSS-INDUSTRY APPROACH

Andrea Bassanini, Andrea Garnero, Pascal Marianna, Sebastien Martin (2010)

No. 106 RISING YOUTH UNEMPLOYMENT DURING THE CRISIS: HOW TO PREVENT NEGATIVE LONG-TERM CONSEQUENCES ON A GENERATION?

Stefano Scarpetta, Anne Sonnet and Thomas Manfredi (2010) 
No. 105 TRENDS IN PENSION ELIGIBILITY AGES AND LIVE EXPECTANCY, 1950-2050

Rafal Chomik and Edward Whitehouse (2010)

No. 104 ISRAELI CHILD POLICY AND OUTCOMES John Gal, Mimi Ajzenstadt, Asher Ben-Arieh, Roni Holler and Nadine Zielinsky (2010)

No. 103 REFORMING POLICIES ON FOREIGN WORKERS IN ISRAEL Adriana Kemp (2010)

No. 102 LABOUR MARKET AND SOCIO-ECONOMIC OUTCOMES OF THE ARAB-ISRAELI POPULATION Jack Habib, Judith King, Asaf Ben Shoham, Abraham Wolde-Tsadick and Karen Lasky (2010)

No. 101 TRENDS IN SOUTH AFRICAN INCOME DISTRIBUTION AND POVERTY SINCE THE FALL OF APARTHEID

Murray Leibbrandt, Ingrid Woolard, Arden Finn and Jonathan Argent (2010)

No. 100 MINIMUM-INCOME BENEFITS IN OECD COUNTRIES: POLICY DESIGN, EFFECTIVENESS AND CHALLENGES Herwig Immervoll (2009)

No. 99 HAPPINESS AND AGE CYCLES - RETURN TO START...? ON THE FUNCTIONAL RELATIONSHIP BETWEEN SUBJECTIVE WELL-BEING AND AGE Justina A.V. Fischer (2009)

No. 98 ACTIVATION POLICIES IN FINLAND

Nicola Duell, David Grubb and Shruti Singh (2009)

No. 97 CHILDREN OF IMMIGRANTS IN THE LABOUR MARKETS OF EU AND OECD COUNTRIES: AN OVERVIEW

Thomas Liebig and Sarah Widmaier (2009)

A full list of Social, Employment and Migration Working Papers is available at www.oecd.org/els/workingpapers.

Other series of working papers available from the OECD include: OECD Health Working Papers. 


\section{RECENT RELATED OECD PUBLICATIONS:}

EQUAL OPPORTUNITIES? The Labour Market Integration of the Children of Immigrants (2010), via OECD Bookshop OECD REVIEWS OF LABOUR MARKET AND SOCIAL POLICIES: ESTONIA (2010), www.oecd.org/els/estonia2010 JOBS FOR YOUTH: GREECE (2010), www.oecd.org/employment/youth JOBS FOR YOUTH: DENMARK (2010), www.oecd.org/employment/youth OECD REVIEWS OF LABOUR MARKET AND SOCIAL POLICIES: ISRAEL (2010), www.oecd.org/els/israel2010

JOBS FOR YOUTH: UNITED STATES (2009), www.oecd.org/employment/youth JOBS FOR YOUTH: POLAND (2009), www.oecd.org/employment/youth OECD EMPLOYMENT OUTLOOK: Tackling the Jobs Crisis (2009), www.oecd.org/els/employment/outlook DOING BETTER FOR CHILDREN (2009), www.oecd.org/els/social/childwellbeing SOCIETY AT A GLANCE - ASIA/PACIFIC EDITION (2009), www.oecd.org/els/social/indicators/asia OECD REVIEWS OF LABOUR MARKET AND SOCIAL POLICIES: SLOVENIA (2009), www.oecd.org/els/slovenia2009 INTERNATIONAL MIGRATION OUTLOOK: SOPEMI (2010) www.oecd.org/els/migration/imo

PENSIONS AT A GLANCE 2009: Retirement-Income Systems in OECD Countries (2009), www.oecd.org/els/social/pensions/PAG

JOBS FOR YOUTH: FRANCE (2009), www.oecd.org/employment/youth SOCIETY AT A GLANCE 2009 - OECD Social Indicators (2009), www.oecd.org/els/social/indicators/SAG JOBS FOR YOUTH: AUSTRALIA (2009), www.oecd.org/employment/youth OECD REVIEWS OF LABOUR MARKET AND SOCIAL POLICIES: CHILE (2009), www.oecd.org/els/chile2009 PENSIONS AT A GLANCE - SPECIAL EDITION: ASIA/PACIFIC (2009),www.oecd.org/els/social/pensions/PAG SICKNESS, DISABILITY AND WORK: BREAKING THE BARRIERS (VOL. 3) - DENMARK, FINLAND, IRELAND AND THE NETHERLANDS (2008), www.oecd.org/els/disability GROWING UNEQUAL? Income Distribution and Poverty in OECD Countries (2008), www.oecd.org/els/social/inequality JOBS FOR YOUTH: JAPAN (2008), www.oecd.org/employment/youth JOBS FOR YOUTH: NORWAY (2008), www.oecd.org/employment/youth JOBS FOR YOUTH: UNITED KINGDOM (2008), www.oecd.org/employment/youth JOBS FOR YOUTH: CANADA (2008), www.oecd.org/employment/youth JOBS FOR YOUTH: NEW ZEALAND (2008), www.oecd.org/employment/youth JOBS FOR YOUTH: NETHERLANDS (2008), www.oecd.org/employment/youth For a full list, consult the OECD online Bookshop at www.oecd.org/bookshop. 